\author{
Universidade de São Paulo \\ Instituto de Física
}

\title{
Física Estatística de Compressed Sensing Online
}

\author{
Paulo Victor Camargo Rossi
}

Orientador: Prof. Dr. Renato Vicente

Tese de doutorado apresentada ao Instituto de Física da Universidade de São Paulo, como requisito parcial para a obtenção do título de Doutor em Ciências.

Banca Examinadora:

Prof. Dr. Renato Vicente (IME-USP)

Prof. Dr. Nestor Felipe Caticha Alfonso (IF-USP)

Prof. Dr. Masayuki Oka Hase (EACH-USP)

Prof. Dr. Tânia Tomé Martins de Castro (IF-USP)

Prof. Dr. Adriano Polpo de Campos (DEs-UFSCar)

São Paulo 


\section{FICHA CATALOGRÁFICA}

\section{Preparada pelo Serviço de Biblioteca e Informação}

do Instituto de Física da Universidade de São Paulo

Rossi, Paulo Victor Camargo

Física estatística de compressed sensing online. São Paulo, 2018.

Tese (Doutorado) - Universidade de São Paulo. Instituto de Física. Depto. de Física Geral

Orientador: Prof. Dr. Renato Vicente

Área de Concentração: Física

Unitermos: 1. Física estatística; 2. Inferência Bayesiana;

3. Inferência estatística; 4 . Computação aplicada. 


\title{
University of São Paulo \\ Physics Institute
}

\section{Statistical Physics of Online Compressed Sensing}

\author{
Paulo Victor Camargo Rossi
}

Supervisor: Prof. Dr. Renato Vicente

Thesis submitted to the Physics Institute of the University of São Paulo in partial fulfillment of the requirements for the degree of Doctor of Science.

Examining Committee:

Prof. Dr. Renato Vicente (IME-USP)

Prof. Dr. Nestor Felipe Caticha Alfonso (IF-USP)

Prof. Dr. Masayuki Oka Hase (EACH-USP)

Prof. Dr. Tânia Tomé Martins de Castro (IF-USP)

Prof. Dr. Adriano Polpo de Campos (DEs-UFSCar) 



\section{ACKNOWLEDGEMENTS}

First I'd like to thank all that were involved in the whole process that led to this thesis, either in a direct or indirect manner. In particular, Prof. Renato Vicente for all the guidance during the many years I have studied under his supervision, and Prof. Yoshiyuki Kabashima for having welcomed me so warmly in Tokyo during my not-so-short stay there. I'd like to thank both of them for all inputs provided, all experiences shared, and their supportive attitudes at all times. Thanks also to Prof. Nestor Caticha for the always exciting and knowledgeable talks and to Tomoyuki Obuchi for all the help in not-so-trivial matters of Japanese daily life.

To all my friends and family, whose friendship withstood the (sometimes long) periods of absence. To André and João Pedro for sharing the burden of many years of parallel studies. To everyone at the Physics Institute and at the Tokyo Institute of Technology who helped in some way or another, be it academically or with meaningful talks about life and everything else. To the people at the Latam Experian DataLab, whose auspicious environment led to productive research and to one of the works described here.

To all those that during these years became or remained important parts of my life - I sincerely hope you can all see a little bit of you in the almost endless calculations that follow.

Financial support from the National Council for Scientific and Technological Development (CNPq) under grant 141737/2013-1 and from the São Paulo Research Foundation under grants 2014/00792-7 and 2014/22258-2 is acknowledged. Material support was also provided by the Center for Natural and Artificial Information Processing Systems (CNAIPS-USP). 

To Priscilla, for everything. 

"Tiger got to hunt, bird got to fly;

Man got to sit and wonder 'why, why, why?'

Tiger got to sleep, bird got to land;

Man got to tell himself he understand."

— Kurt Vonnegut Jr. 



\section{ABstraCt}

In this work, Compressed Sensing is introduced from a Statistical Physics point of view. Following a succinct introduction where the basic concepts of the framework are presented, including necessary measurement conditions and basic signal reconstruction methods, the typical performance of the Bayesian reconstruction scheme is analyzed through a replica calculation shown in pedagogical detail. Thereafter, the main original contribution of this work is introduced - the Bayesian Online Compressed Sensing algorithm makes use of a mean-field approximation to simplify calculations and reduce memory and computation requirements, while maintaining the asymptotic reconstruction accuracy of the offline scheme in the presence of additive noise. The last part of this work are two extensions of the online algorithm that allow for optimized signal reconstruction in the more realistic scenarios where perfect knowledge of the generating distribution is unavailable.

Keywords: Compressed Sensing; Bayesian inference; Statistical Physics; Replicas; Online algorithms 



\section{RESUMO}

Neste trabalho, Compressed Sensing é introduzido do ponto de vista da Física Estatística. Após uma introdução sucinta onde os conceitos básicos da teoria são apresentados, incluindo condições necessárias para as medições e métodos básicos de reconstrução do sinal, a performance típica do esquema Bayesiano de reconstrução é analisada através de um cálculo de réplicas exposto em detalhe pedagógico. Em seguida, a principal contribuição original do trabalho é introduzida — o algoritmo Bayesiano de Compressed Sensing Online faz uso de uma aproximação de campo médio para simplificar cálculos e reduzir os requisitos de memória e computação, enquanto mantém a acurácia de reconstrução do esquema offline na presença de ruído aditivo. A última parte deste trabalho contém duas extensões do algoritmo online que permitem reconstrução otimizada do sinal no cenário mais realista onde conhecimento perfeito da distribuição geradora não está disponível.

Palavras-chave: Compressed Sensing; Inferência Bayesiana; Física Estatística; Réplicas; Algoritmos Online 



\section{Notation}

Sets, Vectors and Matrices

$x, y, z$

Scalars

$\boldsymbol{x}=\left(x_{i}\right), \boldsymbol{y}=\left(y_{i}\right), \boldsymbol{z}=\left(z_{i}\right)$

Vectors

$\underline{A}, \underline{B}, \underline{C}$

Matrices

$x_{i}$

$i$-th component of vector $\boldsymbol{x}$

$A_{i}^{\mu} \equiv A_{\mu i}$

Element at the $\mu$-th row and $i$-th

column of matrix $\underline{A}$

$\boldsymbol{x}_{\backslash i}=\left(x_{1}, \ldots, x_{i-1}, x_{i+1}, \ldots, x_{N}\right)$

All components of $\boldsymbol{x}$ except $x_{i}$

Functions And RANDom VARIABles

$$
\begin{aligned}
& f(x) \propto g(x) \\
& \log (x) \\
& \mathcal{D} x=\frac{1}{\sqrt{2 \pi}} e^{-x^{2} / 2} d x \\
& H(x)=\int_{x}^{\infty} \mathcal{D} z \\
& x \sim P(x) \\
& \boldsymbol{x} \sim P(\boldsymbol{x}) \\
& P(x \mid I) \\
& P(x ; \boldsymbol{\theta}) \\
& \mathcal{N}\left(\bar{x}, \sigma^{2}\right) \equiv \mathcal{N}\left(x ; \bar{x}, \sigma^{2}\right)=\frac{1}{\sqrt{2 \pi \sigma^{2}}} e^{-\frac{1}{2 \sigma^{2}}(x-\bar{x})^{2}} \\
& \langle f(x)\rangle_{P(x)}=\int d x f(x) P(x)
\end{aligned}
$$

$f(x)=k g(x)$ for some constant $k$ Natural logarithm $\log _{e}(x)$ Gaussian measure

Complementary error function $x$ is a random variable distributed according to $P(x)$ $\boldsymbol{x}$ is a random vector distributed according to $P(\boldsymbol{x})$ Probability distribution of $x$ conditioned on $I$ Probability distribution of $x$ parameterized by $\boldsymbol{\theta}=\left(\theta_{i}\right)$ Normal distribution with mean $\bar{x}$ and variance $\sigma^{2}$ Expected value of $f(x)$ with respect to 



\section{Contents}

$\begin{array}{ll}\text { Introduction } & 17\end{array}$

1 Compressed Sensing 25

1.1 Introduction . . . . . . . . . . . . . . . . 26

1.2 Basic signal reconstruction: LASSO . . . . . . . . . . . . . 27

1.3 Building a sensing matrix . . . . . . . . . . . . . . 31

1.4 CS in action (1): Merging the concepts . . . . . . . . . . . 33

1.5 CS in action (2): Single-pixel camera . . . . . . . . . . . 38

2 The Bayesian approach and Statistical Physics 43

2.1 Introduction . . . . . . . . . . . . . . . . . . . . . . . 44

2.2 Statistical Physics and typical performance . . . . . . . . . . . . . 48

2.3 Replica calculation for the Bayesian reconstruction . . . . . . . . . 51

3 Online Compressed Sensing $\quad 67$

3.1 Introduction . . . . . . . . . . . . . . . . . 68

3.2 Online learning . . . . . . . . . . . . . . . . . . . . . . 69

3.3 Bayesian Online Compressed Sensing algorithm . . . . . . . . . . 75

4 Mismatched priors $\quad \mathbf{9 7}$

4.1 Introduction . . . . . . . . . . . . . . . . . . . . . . . 98

4.2 Parameter learning . . . . . . . . . . . . . . . . . . 100

$4.3 \ell_{1}$-minimization algorithm for Bayesian Online CS . . . . . . . . . 106

$\begin{array}{lr}\text { Conclusion } & 113\end{array}$

Appendix A Belief Propagation and the AMP algorithm 115

$\begin{array}{ll}\text { Appendix B 1-bit Compressed Sensing } & 125\end{array}$

$\begin{array}{lr}\text { References } & 127\end{array}$ 



\section{INTRODUCTION}

It has become commonplace talking about the recent "information explosion" or "data deluge". These expressions refer to a much faster growth in data production compared to all available data storage. The divergence is even more evident if data production is compared to data processing capacity [30]. In 2007, the amount of produced data was for the first time larger than the available storage (264 Exabytes $=264 \times 10^{18}$ bytes). Fast-forward to 2011 and humanity had already produced twice the data that could be stored. This state of things begs for more efficient ways of producing and storing data [62, 63, 119].

The Nyquist rate $^{1}[94,113]$ is a concept present in virtually all signal acquisition protocols used in consumer electronics and medical imaging devices, among others [23]. Unfortunately it implies the necessity of a high sampling frequency [27], which means a especially high demand if we consider the now pervasive high definition registers.

But natural and man-made signals tend to be compressible, which means their innate redundancies allow them to be expressed as a small number of combinations of some elemental components - they are sparse (or quasi-sparse) in some basis. Common examples are digital images which can be transformed from its usual 2D spacial representation to a wavelet representation where a large fraction of its components is very close to zero. In these cases, ignoring these components make little difference to the visual perception of the image (see Figure 2 for a simple example). In fact, compression methods such as JPEG and JPEG2000 are based on similar theoretical grounds [118]. In the last decades a lot of effort has been directed to the development of compression techniques which allow the sampled data to be rewritten to a reduced number of bits. This way, a typical current measurement process usually means the gathering of a lot of redundant (one might even say unnecessary)

\footnotetext{
${ }^{1}$ The works of Nyquist in 1928 [94] and Shannon in 1949 [113] demonstrated that if a signal has a maximum frequency of $B$ Hertz it is completely determined by a set of samples spaced $1 /(2 B)$ seconds apart. Equivalently, in a limited-band channel a signal of maximum frequency $B$ could be exactly reconstructed from uniformly spaced samplings with frequency $f_{s}>2 B$ [27]. This is what is now know as the Nyquist-Shannon sampling theorem or the cardinal theorem of interpolation and the critical sampling rate is called the Nyquist rate.
} 
data followed by a discard of a large part of it through a compression algorithm so it can be more effectively stored or transmitted, implying additional computational costs. Using a digital image as an example once again, a typical camera has millions of imaging sensors (pixels) in its CCD or CMOS sensor but the picture it produces is encoded in just hundreds or thousands of kilobytes. This means superfluous costs on the gathering and processing of the data. When talking about medical imaging which might include radiation, this also means health costs on the long run for the patients.

Between 2004 and 2006, Candès, Tao e Donoho [19, 21, 22, 33, 34] showed that sparse signals could be exactly reconstructed with below-the-Nyquist-rate sampling. Moreover (and perhaps what makes it so intriguing) the reconstruction could be done in non-adaptive fashion. These seminal works gave rise to the family of techniques called Compressed Sensing ${ }^{2}$ (CS) [51]. It should be noted that the word "sampling" has a twist, though - in CS a sample of the signal is obtained by calculating its inner product against different test functions. It is a different paradigm from the usual point sampling. Compressed sensing is a framework for signal gathering and processing which presents itself as a successful resource that has produced many techniques for efficient information extraction [116]. CS utilizes the fact that real-world signals can typically be represented by a small combination of elemental components $[23,64]$ to eliminate the data redundancy directly in its acquisition. In a standard scenario, CS utilizes this property to enable the recovery of signals from much fewer samples (or linear measurements) than required by the Nyquist-Shannon theorem [33, 39, 51].

In its beginnings, the idea of Compressed Sensing was of such novelty that, according to Schulz et al., an earlier paper outlining it was rejected because "its claims appeared impossible to be substantiated" [112]. More than a decade later the field of CS and its many ramifications have gained enormous popularity among scientists of several disciplines (see Figure 1) and several journals have allocated special editions for this and other related topics [49, 116]. As a consequence, the concept of CS is now spreading in various directions. For instance, in a remote sensing situation where a sensor transmits data to a data center placed at a far distance, the amount of data to be sent through a narrow band communication channel may be the biggest hindrance to efficient signal processing - as a practical solution to such a difficulty, 1-bit CS is a proposed scheme for recovering sparse signals by using only the sign information of the measurement results $[14,136,137]$.

\footnotetext{
${ }^{2}$ In the literature, alternative names to Compressed Sensing are Compressed Sampling, Compressive Sensing and Compressive Sampling. Luckily all can be abbreviated to CS.
} 

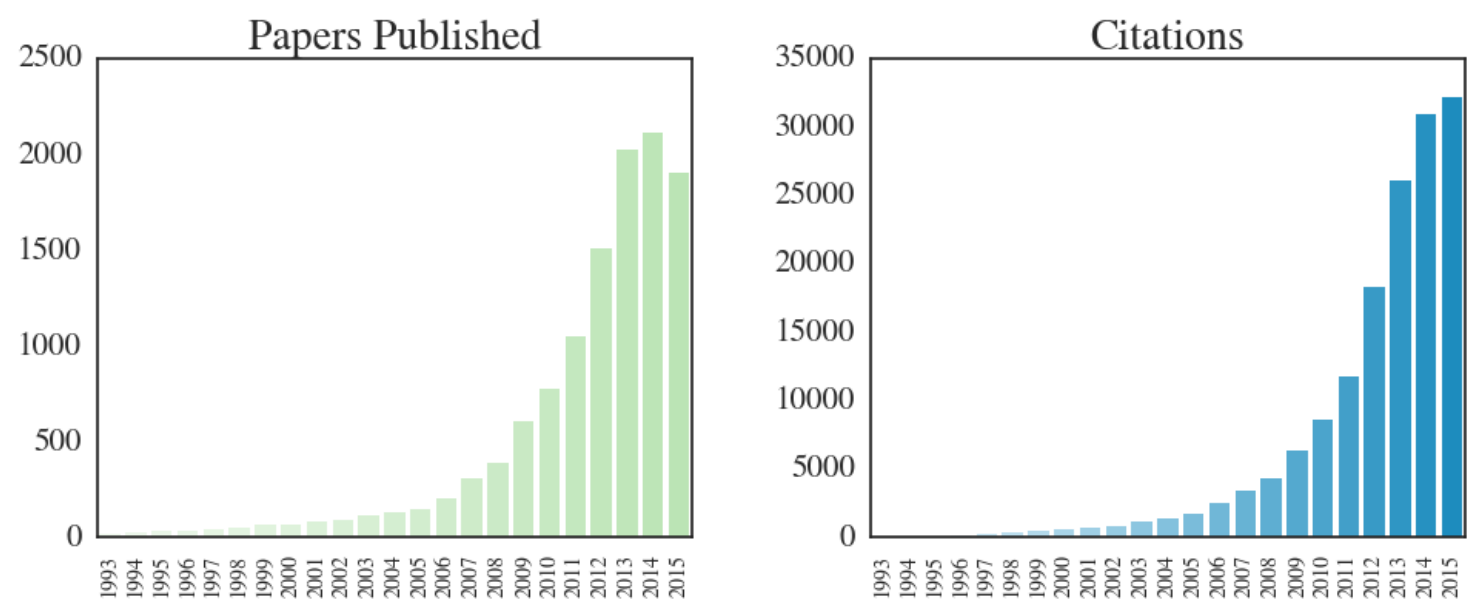

Figure 1: Number of published papers and citations counts for works related to Compressed Sensing. The search was done on the Web of Science database with the string: "TS = ( ((parsim* or spars*) and (represent* or approx* or sol* or convex) and (pursuit or dictionary or transform)) or (compres* and (sens* or sampl*) and (spars* or parsim*)) )". This graph corresponds to an updated version of Figure 2 of [49].

Another variation can be utilized when multiple signals are observed in a distributed manner. In such cases, the signal recovery performance can also be enhanced by exploiting information on correlations among the signals - this is referred to as distributed CS [5, 43, 114, 134].

In this thesis we explore the possibilities and limitations of another variation of CS, which we call Bayesian Online Compressed Sensing. There is a vast literature on Bayesian schemes for CS (e.g. [75, 100, 122]) and their reconstruction have been thoroughly examined, not least by the Statistical Physics community [74, 101, 137]. Here, in the online scheme, to minimize the computation and memory costs as much as possible, measured data are used for signal recovery only once and discarded thereafter. This approach towards information processing is a promising technique for when devices of low computational capability are used for signal recovery; such situations can arise in sensor networks, multi-agent systems, nano-devices, and so on. ${ }^{3}$ It is also advantageous when the signal source is time-variant. Computation intensive tasks such as magnetic resonance imaging where real-time reconstruction is desirable [79] can also be benefited by such a scheme. Another possible application domain is simulation science - recent development of high-performance computing resources significantly increases the size of data produced by computer simulation such as in computational fluid dynamics, making it more and more difficult to store the entire set of raw data in a hard disk [109]. The online scheme would be highly

\footnotetext{
${ }^{3}$ Recently the concept of mini-batch learning [15], which is an interesting compromise between online and offline learning, has also found its way to the CS scenario [81]. This possibility is not considered here.
} 
useful as a technique for extracting fundamental components of the dynamics out of the raw data in situ, which may free us of the limitation of data storage. Historically, online information processing was actively investigated by the physics community more than two decades ago in the context of learning by neural networks [13, 69, $72,95,107,108,126,128]$. We hope this work bridges the gap between those results and Compressed Sensing, arguing for the utility of sparsity knowledge in online Compressed Sensing reconstruction. 


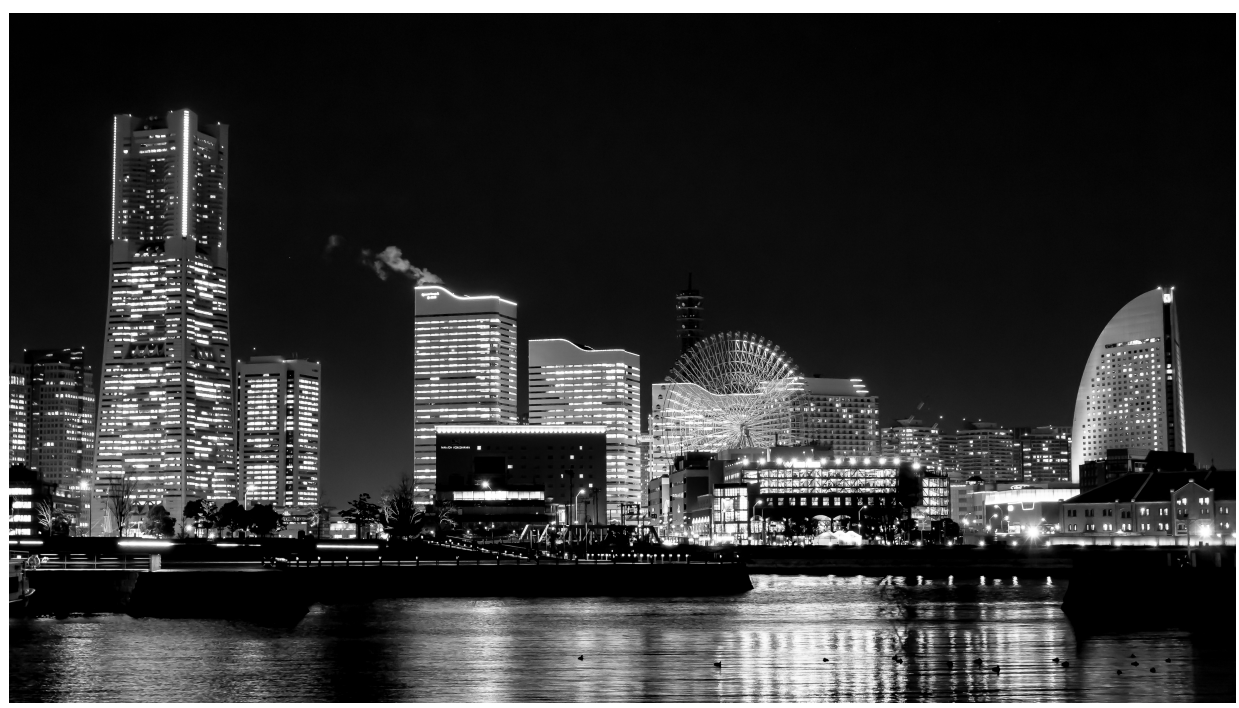

(a) Original image

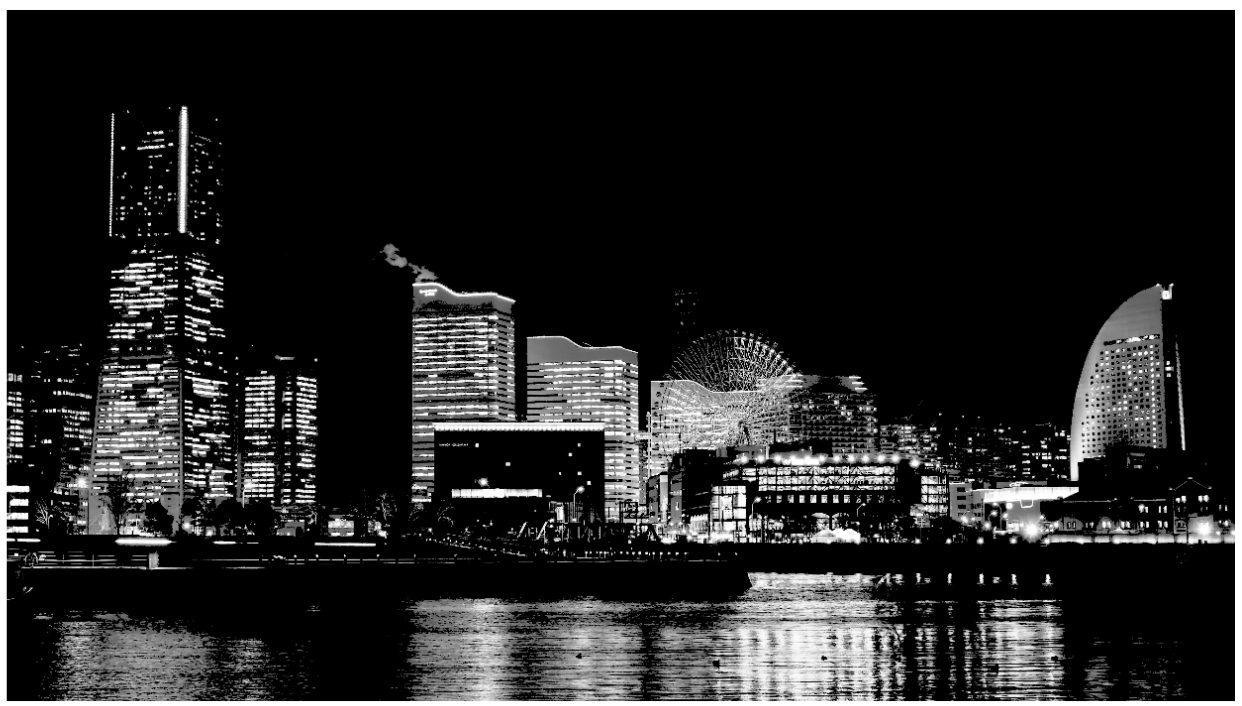

(b) Image after compression

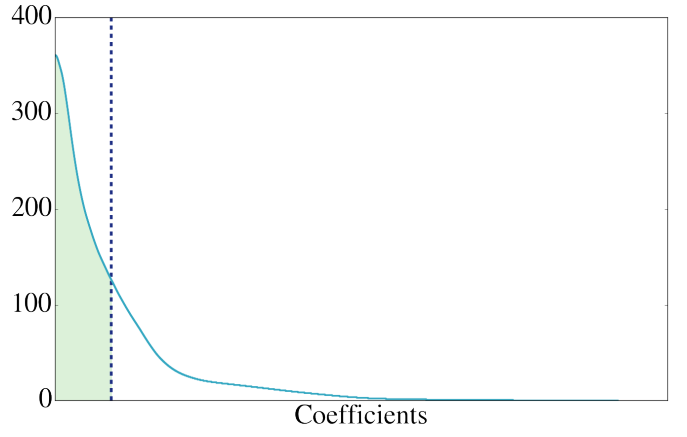

(c) Ordered wavelet coefficients. The vertical line delimits the largest components kept in the compressed image.

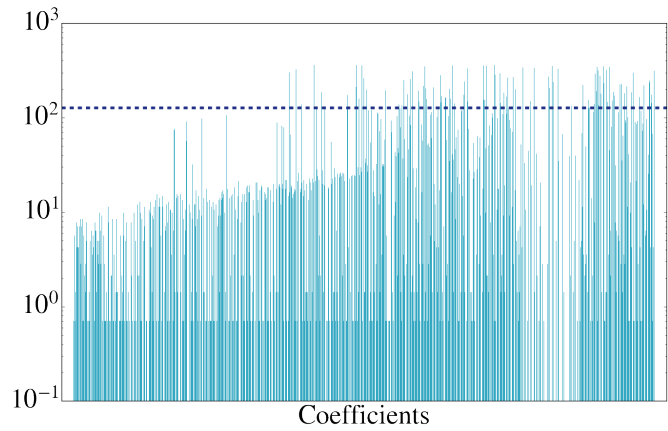

(d) Wavelet coefficients. The horizontal line delimits the minimum coefficient value for which components were kept.

Figure 2: Yokohama (Japan) skyline. The compressed image (b) was obtained from the original image (a) after selection of only its largest 10\% Haar wavelet transform coefficients $((c)$ and $(d))$. All other coefficients were set to zero and a inverse transform produced (b). 



\section{HOW THIS THESIS IS ORGANIZED}

Chapters 1 and 2 provide a pedagogical overview of Compressed Sensing and Statistical Physics.

Chapter 1 introduces Compressed Sensing along with main theoretical results. There is an ever-growing literature on the subject and this thesis does not attempt to cover all the important topics of interest in the Compressed Sensing community. Instead, only results deemed necessary for a broad understanding of the theory will be presented, in order to allow for the introduction of the Bayesian reconstruction scheme along with the Online Compressed Sensing algorithm.

In Chapter 2, Bayesian techniques are introduced and connected to Statistical Physics. A detailed calculation of the typical reconstruction error in the Bayesian reconstruction scheme is obtained through the replica method. There are not many examples in the literature of full calculations of this kind, so there is the expectation that this chapter might prove itself useful for someone tackling a replica calculation for the first time.

Chapters 3 and 4 consist of the two main original contributions of this thesis, each of them the result of a different collaboration.

Together with Professor Yoshiyuki Kabashima of the Tokyo Institute of Technology and in collaboration with the late Professor Jun-ichi Inoue, an online reconstruction scheme for Compressed Sensing was developed. This scheme [105] forms the bulk of Chapter 3 of this thesis. There it is explained as a continuation of the works of Opper and others in Bayesian online learning. A macroscopic analysis of the reconstruction is then provided, with and without measurement noise/information degradation. The macroscopic variables that appear in this section are the same as the replica overlap variables from Ch. 2, allowing direct comparisons to be made.

The Online Compressed Sensing algorithm above performs the best with perfect knowledge of the signal generating distribution (encoded in the prior distribution). To extend this work to more realistic scenarios where this knowledge is often not available, Chapter 4 introduces two alternative techniques for hyper-parameter learning developed in collaboration with Professor Renato Vicente [106]. They both draw heavily from offline reconstruction schemes already available, but their use in an online setting is new, as far as we know. 

Chapter 1

\section{Compressed Sensing}

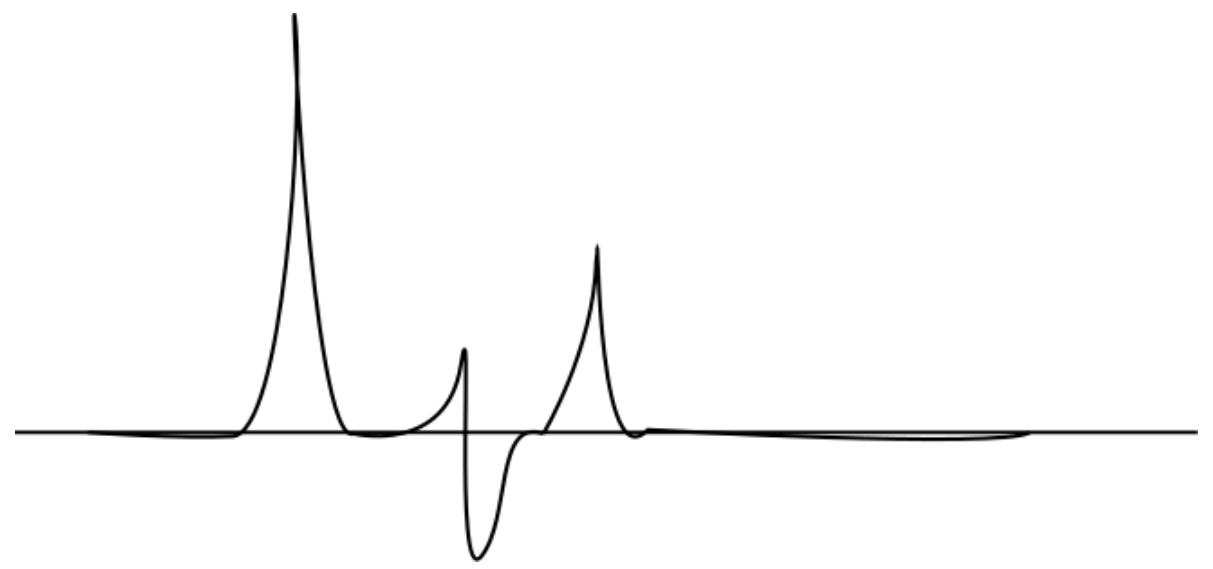




\subsection{INTRODUCTION}

Given a $N$-dimensional signal $\boldsymbol{x}_{0} \in \mathbb{R}^{N}$ with at most $K$ non-zero components ${ }^{1}, M$ linear projections $u_{0}^{\mu}$ are produced according to

$$
u_{0}^{\mu}=\mathbf{A}^{\mu} \cdot \boldsymbol{x}_{0}, \quad \mu=1, \ldots, M .
$$

All measurement vectors $\mathbf{A}^{\mu} \in \mathbb{R}^{N}$ can be chosen at will and are known to us; the projections $u_{0}^{\mu}$, at least as a rule, are not. What we do have access to are the measurements

$$
y^{\mu} \sim P\left(y^{\mu} \mid u_{0}^{\mu}\right)
$$

for $\mu=1, \ldots, M$ (Figure 1.1). The probability distribution $P(y \mid u)$ models the kind of measurement apparatus available and/or its limitations, such as the presence of noise or the modulation of $u$ by whatever function $f(u)$. The simple identity function $f(u)=u$ is by far the most used in theoretical CS problems - its use is so pervasive that this setting became known as Standard $C S .^{2}$ In a noiseless scenario, this can be represented by $P(y \mid u)=\delta(y-u)$, while corruption of information by additive Gaussian noise, i.e. $y=u+\xi$, with $\xi \sim \mathcal{N}\left(0, \sigma_{n}^{2}\right)$ for some variance $\sigma_{n}^{2}$, would correspond to $P(y \mid u)=\exp \left[-(y-u)^{2} /\left(2 \sigma_{n}^{2}\right)\right] / \sqrt{2 \pi \sigma_{n}^{2}}$. In this text, these will be referred to as the noiseless and noisy Standard CS cases, respectively.

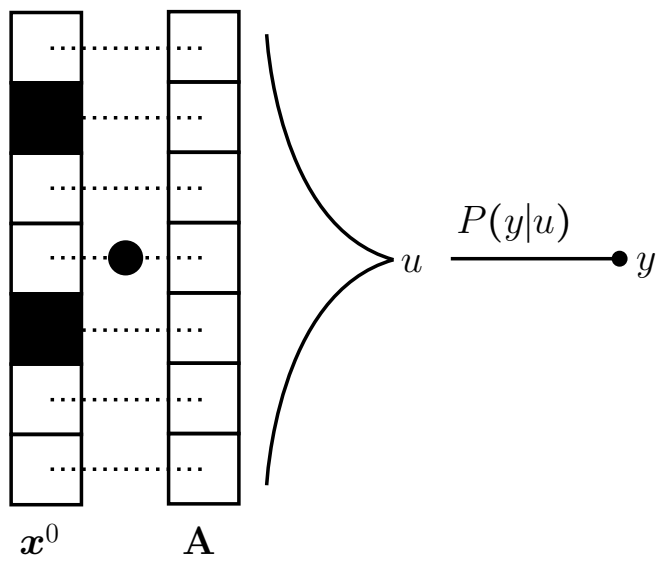

Figure 1.1: Schematic representation of the measurement process in CS. The sparse signal $\boldsymbol{x}_{0}$ is projected onto a (known) random measurement vector $\boldsymbol{A}$. The result $u=\mathbf{A} \cdot \boldsymbol{x}_{0}$ goes through a (possibly noisy) channel $P(y \mid u)$ from which the measurement $y$ is obtained.

Compressed Sensing is a quintessential statistical inference problem. The two stages (in no particular order) of a typical CS problem can be briefly described as:

\footnotetext{
${ }^{1}$ In this case we say $\boldsymbol{x}_{0}$ is a $K$-sparse vector; more commonly, though, it is simply called sparse.

${ }^{2}$ In appendix B a more complex function $f(u)=\operatorname{sgn}(u)$ will be introduced to account for situations of limited storage capacity. This scenario is called 1-bit Compressed Sensing.
} 
- Elaboration of a non-adaptive reconstruction method to recover $\boldsymbol{x}_{0}$ from the measurements $y^{\mu}, \mu=1, \ldots, M$, and the knowledge of all $\mathbf{A}^{\mu}$;

- Construction of a series of measurement vectors $\mathbf{A}^{\mu}$ that makes the measurements meaningful and, therefore, the reconstruction possible and efficient.

The most basic question of the entire research field is: what is the minimum number $M$ of linear projections for which we can recover $\boldsymbol{x}_{0}$ from the data $D^{M}:=$ $\left\{\left(y^{\mu}, \mathbf{A}^{\mu}\right)\right\}_{\mu=1}^{M}$ within some reasonable error threshold? The "compressed" in Compressed Sensing comes from the fact that it has been shown that exact recovery can be done for $M<N$ (for properly chosen vectors $\mathbf{A}^{\mu}$ and for specific reconstruction algorithms) [19, 21, 33].

If all measurement vectors were to be stacked on top of each other, one would obtain a sensing (or measurement) matrix $\underline{A}=\left(A_{i}^{\mu}\right)$. Here, $A_{i}^{\mu}$ is the $i$-th component of $\mathbf{A}^{\mu}$. In this notation, the general Compressed Sensing problem can be stated as a linear system

$$
\boldsymbol{u}=\underline{A} \boldsymbol{x}_{0} .
$$

As mentioned earlier, the construction of the measurements vectors $\mathbf{A}^{\mu}$ (and by consequence the construction of the sensing matrix $\underline{A}$ ) is one of the challenges of CS. In general, anything is allowed, though it quickly becomes obvious that some classes of matrices are more efficient than others. The main interest of this work resides in the first of the two stages described above, i.e. in elaborating on the signal's reconstruction approaches. For this reason, discussions regarding the choices of the sensing matrix will be only briefly discussed.

\subsection{BASIC SIGNAL RECONSTRUCTION: LASSO}

In this section we will consider what is arguably the most relevant signal reconstruction technique for Compressed Sensing - the $\ell_{1}$-norm minimization scheme called LASSO. Besides its historical importance (being at the center of all seminal works) it has interesting geometrical interpretations. Let us begin then with the noiseless Standard CS problem, namely, $\boldsymbol{y}=\underline{A} \boldsymbol{x}_{0}$.

From linear algebra we know that such a system with $M<N$, having more unknowns than equations, has infinitely many solutions. ${ }^{3}$ For any experimenter that would be a major annoyance since their interest is in getting only the solution that would represent the best signal reconstruction possible. In cases like this, a

\footnotetext{
${ }^{3}$ It is assumed here that $\underline{A}$ is a full-rank matrix otherwise it could be that the system would have no solution at all.
} 


$$
\left(\begin{array}{c}
y^{1} \\
y^{2} \\
\vdots \\
\boldsymbol{y}^{\mu} \\
\vdots \\
y^{M-1} \\
y^{M}
\end{array}\right)=\left(\begin{array}{ccccc}
\mathbf{A}_{1}^{1} & \mathbf{A}_{2}^{1} & \ldots & \mathbf{A}_{N-1}^{1} & \mathbf{A}_{N}^{1} \\
\mathbf{A}_{1}^{2} & \mathbf{A}_{2}^{2} & \cdots & \mathbf{A}_{N-1}^{2} & \mathbf{A}_{N}^{2} \\
\vdots & \ddots & & \ddots & \vdots \\
& & \mathbf{A}_{i} & & \\
\vdots & \ddots & & \ddots & \vdots \\
\mathbf{A}_{1}^{M-1} & \mathbf{A}_{2}^{M-1} & \ldots & \mathbf{A}_{N-1}^{M-1} & \mathbf{A}_{N}^{M-1} \\
\mathbf{A}_{1}^{M} & \mathbf{A}_{2}^{M} & \cdots & \mathbf{A}_{N-1}^{M} & \mathbf{A}_{N}^{M}
\end{array}\right)\left(\begin{array}{c}
x_{0,1} \\
x_{0,2} \\
\vdots \\
\boldsymbol{C}_{0, i} \\
\vdots \\
\\
\end{array}\right.
$$

typical approach to narrow the choice to one well-defined solution is to choose a penalty function $J(\boldsymbol{x})$, apply it to the entire set of possible solutions, and choose the vector $\hat{\boldsymbol{x}}$ which is the least penalized of all. This can be defined as a general optimization problem $\left(P_{J}\right)$ :

$$
\left(P_{J}\right): \min _{\boldsymbol{x}} J(\boldsymbol{x}) \quad \text { s.t. } \quad \boldsymbol{y}=\underline{A} \boldsymbol{x},
$$

where the choice of the penalty $J(\cdot)$ will be determinant to define what solutions $\hat{\boldsymbol{x}}$ we might obtain. The constraint $\boldsymbol{y}=\underline{A} \boldsymbol{x}$ to the right of the expression guarantees that we will only obtain solutions $\hat{\boldsymbol{x}}$ that satisfy all available measurements. To give a concrete example, a particularly common choice for $J(\cdot)$ in problems of this kind (and especially in various fields of engineering) is the Euclidean norm $\ell_{2}(\boldsymbol{x}) \equiv \sqrt{\sum_{i=1}^{N}\left|x_{i}\right|^{2}}$. Its widespread use lies in its simplicity - the resulting optimization has a closed-form solution which correspond to the pseudo-inverse solution $\hat{\boldsymbol{x}}=\underline{A}^{T}\left(\underline{A A^{T}}\right)^{-1} \boldsymbol{y}$. This solution also has the mathematically desirable property of uniqueness, which results directly from the use of a convex penalty function.

Norms ${ }^{4}$ other than the Euclidian $\ell_{2}$ can be defined for $p \geq 1$ as

$$
\ell_{p}(\boldsymbol{x}):=\left(\sum_{i}\left|x_{i}\right|^{p}\right)^{1 / p}
$$

\footnotetext{
with the properties [98]:

For all $a \in F$ and all $u, v$ in $V$,

(a) (absolute scalability) $h(a v)=|a| h(v)$,

(b) (triangle inequality) $h(u+v) \leq h(u)+h(v)$,

(c) If $h(v)=0$ then $v=0$.
}

${ }^{4}$ A norm on the vector space $V$ over a subfield $F$ of the complex numbers is a function $h: V \rightarrow \mathbf{R}$ 
These so-called $\ell_{p}$-norms are also convex. But of even more interest for this text is the same expression above, but for $0<p<1$, even though in these cases it does not correspond to real norms ${ }^{5}$. With this in mind, we define $\left(P_{p}\right)$ as the optimization problem $\left(P_{J}\right)$ in which $J(\cdot)=\ell_{p}(\cdot)$.

Now, to see how the solutions to $\left(P_{p}\right)$ may have very different properties for different $p$, suppose we are given a point $\boldsymbol{z}_{0}$ and we want to find the best approximation to it that resides in some affine space ${ }^{6} \mathbb{F}$ minimizing the norm $\ell_{p}$, i.e. we want to find $\hat{\boldsymbol{z}} \in \mathbb{F}$ that minimizes $\left\|\hat{\boldsymbol{z}}-\boldsymbol{z}_{0}\right\|_{p}:=\ell_{p}\left(\hat{\boldsymbol{z}}-\boldsymbol{z}_{0}\right)$. The best approximation to $\boldsymbol{z}_{0}$ in $\mathbb{F}$ can be found by growing a $\ell_{p}$-sphere centered on $\boldsymbol{z}_{0}$ until it intersects with $\mathbb{F}$ - this will be the point where $\left\|\hat{\boldsymbol{z}}-\boldsymbol{z}_{0}\right\|_{p}$ is minimum. Figure 1.2 shows this procedure in two dimensions, in which the affine space $\mathbb{F}$ is a straight line. It is possible to see that the solutions for different $p$ have very different characteristics - typically, minimizing the norm $\ell_{2}$ results in finding solutions in which the approximation error is spread among the two axis; on the other hand, norms $\ell_{1}$ and $\ell_{1 / 2}$ return approximations with some coordinates set to zero - if you may, sparse approximations of $\boldsymbol{z}_{0}$. In fact, the solution to $\left(P_{p}\right)$ with any $p \leq 1$ tends to be sparse. Clearly, the limiting case is the use of the "counting norm" here defined as $\ell_{0}(\boldsymbol{x})=\lim _{p \rightarrow 0} \ell_{p}(\boldsymbol{x}):=\#\left(i: x_{i} \neq 0\right)$, i.e. the number of elements different from zero $^{7}$. Its use as a penalty function results in the sparsest solution of all.

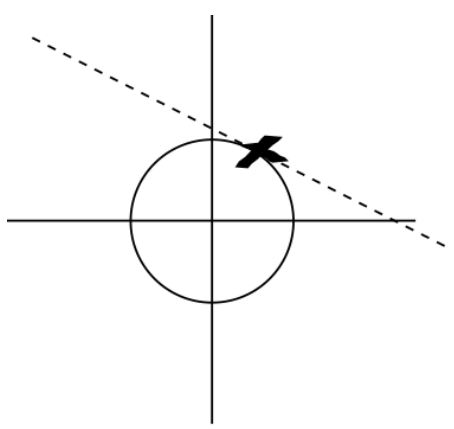

(a) $p=2$

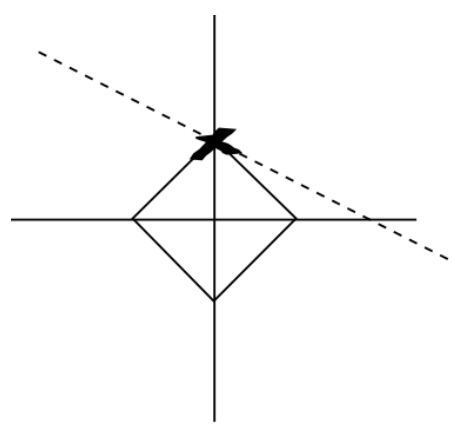

(b) $p=1$

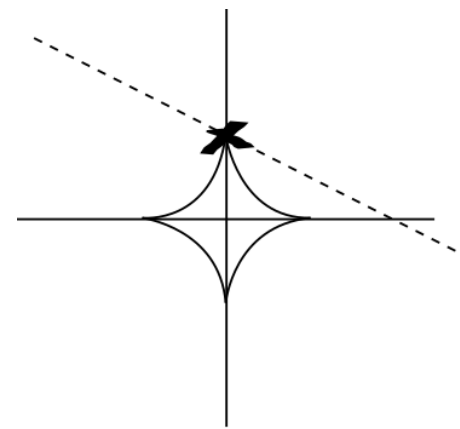

(c) $p=1 / 2$

Figure 1.2: $\ell_{p}$-spheres in two dimensions. The crosses mark the best approximations for $\boldsymbol{z}_{0}$ (origin) in the affine spaces $\mathbb{F}$ (dashed lines), i.e. the intersections between $\mathbb{F}$ and the spheres. Note how for $p \leq 1$ the best approximation lies over one of the axes, so that one of the $\boldsymbol{z}_{0}$ components is correctly identified as zero; for $p=2$ the approximation error is split between the two components.

Therefore, since Compressed Sensing deals with sparse signals, a seemingly natu-

\footnotetext{
${ }^{5}$ When $0<p<1$, definition (1.5) violates the triangle inequality.

6 "An affine space is nothing more than a vector space whose origin we try to forget about, by adding translations to the linear maps."[11]

${ }^{7}$ This one is also not a real norm since it does not satisfy the scalability property: for $t>0$, $\|t \boldsymbol{x}\|_{0}=\|\boldsymbol{x}\|_{0} \neq t\|\boldsymbol{x}\|_{0}[48]$.
} 
ral approach would be to attempt to recover $\boldsymbol{x}_{0}$ by solving the optimization problem $\left(P_{0}\right)$ :

$$
\left(P_{0}\right): \quad \min _{\boldsymbol{x}}\|\boldsymbol{x}\|_{0} \quad \text { s.t. } \quad \boldsymbol{y}=\underline{A} \boldsymbol{x},
$$

after all, by definition, solutions to this optimization would be the sparsest possible. An interesting parallel [99] can be drawn between this scenario and the Occam's razor, which is often loosely stated as the maxim "entities should not be multiplied beyond necessity". Here, this would be something like "one should not consider more non-zero elements than necessary to explain all the measurements". While the viability of $\left(P_{0}\right)$ has been considered for many years, it poses many challenges that have inhibited its widespread use. For example, it is very hard to prove uniqueness of solutions except for very specific matrices $\underline{A}$ and even when a possible solution is found, it is not easy to perform any tests to verify that this solution is actually the global minimum [48]. In fact, for a general matrix $\underline{A}$, to even find an approximation to the true minimum is a NP-hard problem of exponential complexity which requires brute force and exhaustive searches over all subsets of columns of $\underline{A}$. [89, 22]. For these reasons, and considering that all norms $\ell_{p}$ for $0<p<1$ are also not convex, the convex relaxation problem $\left(P_{1}\right)$, known as Basis Pursuit (BP) [25],

$$
\left(P_{1}\right) \text { or }(\mathrm{BP}): \quad \min _{\boldsymbol{x}}\|\boldsymbol{x}\|_{1} \quad \text { s.t. } \quad \boldsymbol{y}=\underline{A} \boldsymbol{x},
$$

becomes a particularly interesting choice for Compressed Sensing.

Now let us consider the more realistic setting of noisy standard CS, i.e. $\boldsymbol{y}=$ $\underline{A} \boldsymbol{x}_{0}+\boldsymbol{\xi}$ where each noise component $\xi_{i}, i=1, \ldots, M$, is sampled from a Gaussian distribution with zero mean and variance $\sigma_{n}^{2}$. In this case, one cannot expect to find a solution $\hat{\boldsymbol{x}}$ that satisfies the hard constraint $\boldsymbol{y}=\underline{A} \hat{\boldsymbol{x}}$; nevertheless, it is indeed possible to find $\hat{\boldsymbol{x}}$ such that $\boldsymbol{y}$ and $\underline{A} \boldsymbol{x}$ are close enough. Start by establishing a thresold $\varepsilon$ such that any vector $\boldsymbol{x}$ that satisfy $\|\boldsymbol{y}-\underline{A} \boldsymbol{x}\|_{2}^{2}<\varepsilon$ is a possible solution. This relaxation in the constraint gives rise to an alternate version of the optimization problem $\left(P_{1}\right)$ called Basis Pursuit Denoising (BPDN) [25]:

$$
(\mathrm{BPDN}): \quad \min _{\boldsymbol{x}}\|\boldsymbol{x}\|_{1} \quad \text { s.t. }\|\boldsymbol{y}-\underline{A} \boldsymbol{x}\|_{2}^{2}<\varepsilon
$$

If one exchanges the location of the constraint, the BPDN formulation is completely equivalent to the LASSO (Least Absolute Shrinkage and Selection Operator) ${ }^{8}$ [120]:

\footnotetext{
${ }^{8}$ There is an interesting historical trivia mentioned by Elad [48]:

"LASSO was conceived by Friedman, Hastie, and Tibshirani, three researchers from the statistic department at Stanford University. This took place around the very same time the Basis-Pursuit was invented for signal processing purposes by Chen,
} 


$$
\text { (LASSO) : } \quad \min _{\boldsymbol{x}}\|\boldsymbol{y}-\underline{A} \boldsymbol{x}\|_{2}^{2} \quad \text { s.t. } \quad\|\boldsymbol{x}\|_{1}<S
$$

or, in its Lagrangian formulation,

$$
\left(\mathrm{LASSO}^{\prime}\right): \quad \min _{\boldsymbol{x}}\|\boldsymbol{y}-\underline{A} \boldsymbol{x}\|_{2}^{2}+\lambda\|\boldsymbol{x}\|_{1}
$$

Here, one is strictly minimizing the squared reconstruction error $\|\boldsymbol{y}-\underline{A} \boldsymbol{x}\|_{2}^{2}$, but the regularization term $\lambda\|\boldsymbol{x}\|_{1}$ enforces sparsity. Of course, the shrinkage parameter $\lambda$ must be chosen appropriately since its value is highly influential on the reconstructed signal - there is a direct relation between $\lambda$ and the number of non-zero coefficients in the estimate, which is reflected on the prediction accuracy and parsimony [47]. The choice of $\lambda$ is a common practical issue that has been answered in several different ways which include calculation of its best value through cross-validation procedures [120] or direct calculation of an ideal number of non-zero covariates by adding one at a time in a recursive manner [47].

\subsection{BuILDing A SENSING MATRIX}

In fact, $\ell_{1}$-minimization programs have a long history that goes back at least to the 1970s [26, 110], but it was only in the 2000s that the exact equivalence of $\left(P_{0}\right)$ and $\left(P_{1}\right)$ was demonstrated $[35,50,59,123]$. This equivalence must be understood properly and, for that, it is necessary to introduce the Restricted Isometry Property (RIP) [20]. 9

A matrix $\underline{A}$ satisfies the $R I P$ of order $k$ if there exists a constant $\delta_{k} \in(0,1)$ such that

$$
\left(1-\delta_{k}\right)\|\boldsymbol{x}\|_{2}^{2} \leq\|\underline{A} \boldsymbol{x}\|_{2}^{2} \leq\left(1+\delta_{k}\right)\|\boldsymbol{x}\|_{2}^{2}
$$

holds for all $k$-sparse vectors $\boldsymbol{x}$ (i.e., vectors with at most $k$ non-zero coordinates). Essentially, the definition means any matrix $\underline{A}$ that satisfies the RIP of order $k$ would approximately preserve the norm of a $k$-sparse vector (alternatively, it would preserve the distance between two $(k / 2)$-sparse vectors). Now assume that $\delta_{2 k}<$ $\sqrt{2}-1$. Theorem 1.2 of [20] affirms that the solution $\hat{\boldsymbol{x}}$ of $\left(P_{1}\right)$ obeys both

$$
\left\|\hat{\boldsymbol{x}}-\boldsymbol{x}_{0}\right\|_{1} \leq C_{0}\left\|\boldsymbol{x}_{0}-\boldsymbol{x}_{k}\right\|_{1}
$$

Donoho, and Saunders — from the same department (actually, the same corridor!!)."

${ }^{9}$ The following remarks follow closely the work of Candès [20] and Candès and Tao [22]. 
and

$$
\left\|\hat{\boldsymbol{x}}-\boldsymbol{x}_{0}\right\|_{2} \leq C_{0} k^{-1 / 2}\left\|\boldsymbol{x}_{0}-\boldsymbol{x}_{k}\right\|_{1},
$$

where $\boldsymbol{x}_{k}$ is an approximation of $\boldsymbol{x}_{0}$ in which only its $k$ largest components were maintained - all other $N-k$ smallest components set to zero. Also above, the constant $C_{0}$ is given by $C_{0}=2\left[1-(1-\sqrt{2}) \delta_{2 k}\right] /\left[1-(1+\sqrt{2}) \delta_{2 k}\right]$. Note the important corollary: if $\boldsymbol{x}_{0}$ is $k$-sparse itself, then provided that $\underline{A}$ satisfies the RIP of order $2 k$, the signal can be recovered exactly. Otherwise, the reconstruction error is bounded by the $\ell_{1}$-distance between the original signal $\boldsymbol{x}_{0}$ and its best sparse approximation $\boldsymbol{x}_{k}$. And now the equivalence of $\left(P_{0}\right)$ and $\left(P_{1}\right)$ can be effectively explained. The results above demonstrate that whenever $\delta_{2 k}<\sqrt{2}-1$, the solution to the $\ell_{1}$-minimization problem is exactly the same as the one for the $\ell_{0}$-minimization problem [20].

A similar result can be obtained for the relaxed threshold minimization BPDN. Consider once again the constant $\delta_{2 k}<\sqrt{2}-1$ and now also the additive noise $\boldsymbol{\xi}$ constraint $\|\boldsymbol{\xi}\|_{2} \leq \varepsilon$. The solution to the BPDN problem obeys

$$
\left\|\hat{\boldsymbol{x}}-\boldsymbol{x}_{0}\right\|_{2} \leq C_{0} k^{-1 / 2}\left\|\boldsymbol{x}_{0}-\boldsymbol{x}_{k}\right\|_{1}+C_{1} \varepsilon,
$$

where $C_{1}=4\left[\sqrt{1+\delta_{2 k}}\right] /\left[1-(1+\sqrt{2}) \delta_{2 k}\right]$.

Although brief, the discussion above makes clear the importance of the RIP most guarantees for the success of reconstructions schemes such as the ones presented in the previous section are stated in terms of this property ${ }^{10}$. In fact, these results gave rise to what is now the entire field of Compressed Sensing. But the challenge remains of building such a matrix. In this text it will be assumed that random matrices with each individual element sampled from a Gaussian distribution satisfy all necessary conditions for the validity of the results above with overwhelming probability. This is correct [51] but will not be proved here. The interested reader is directed to [22] for a rigorous proof. Therefore, in the remainder of the text all elements of the sensing matrices $\underline{A}$ will be considered as independent samples from a Gaussian distribution, i.e. $A_{i}^{\mu} \sim \mathcal{N}\left(0, N^{-1}\right)$, unless explicitly stated otherwise. The simplicity of these matrices has made their use standard in the field, so that the results obtained here will be of sufficient generality.

\footnotetext{
${ }^{10}$ Sometimes, they can also be posed as consequences of some other related concepts, such as the coherence of a matrix — the largest absolute inner product between any of its columns [18] -, or its spark — the smallest number of linearly dependent columns it has [35].
} 


\subsection{CS in ACtion (1): MERGing THE CONCEPTS}

In this section we will review the theoretical considerations presented above regarding the reconstruction algorithms and the construction of sensing matrices in light of numerical simulations.

Consider first a typical sparse signal $\boldsymbol{x}_{0}$ (Figure 1.3), in which only $k$ of the $N$ total components are different from zero. With sufficient generality, we say each of these non-zero components is a sample of some distribution $g(x)$, i.e. $x_{0, i} \sim g\left(x_{0, i}\right)$. It is assumed (and implicitly stated) that all signal components are independent from each other. This is of course a simplification (see any real-life photograph for a counter-example), but one very useful in this context - any measurable dependence could be taken into consideration to strengthen a reconstruction algorithm and obtain even better results $[41,56,111]$. In a sense, this independence assumption guarantees we are working with the most difficult situation.

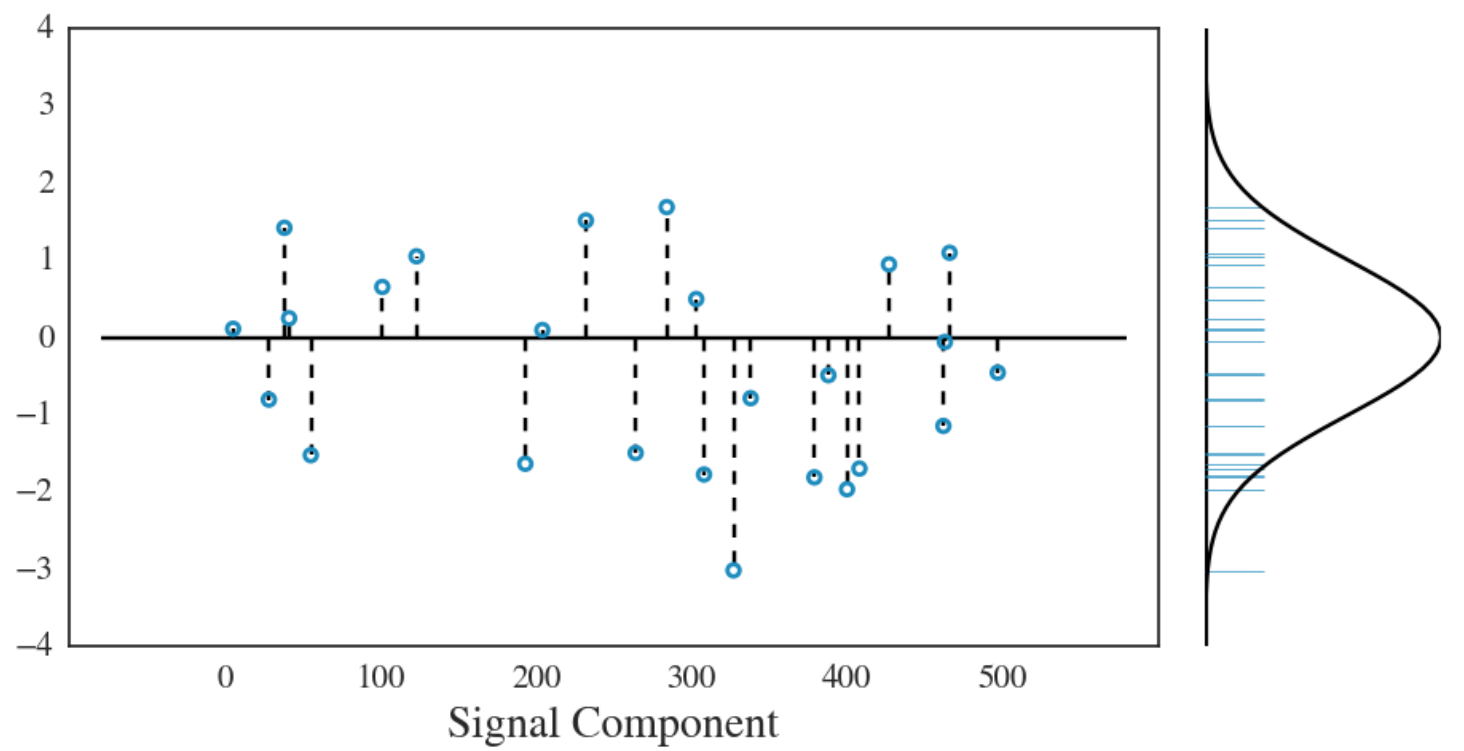

Figure 1.3: Typical sparse signal example. Here the total length of $\boldsymbol{x}_{0}$ is $N=500$. Each of its $k=25$ (5\%) non-zero components are sampled from a Gaussian distribution centred on zero with variance 1 , i.e. $x_{0, i} \sim \mathcal{N}(0,1)$ (shown rotated in the right). For easier visualization, the actual value of each non-zero component is circled in blue while all null components are found over the line.

Now, it may be already clear that the number of measurements affects directly the quality of the signal recovery. Indeed, for a series of $M$ properly chosen measurement vectors $\mathbf{A}^{\mu}$ and noiseless measurements, in the limit where $M=0$ no information at all is obtained while for $M=N$ the system $\boldsymbol{y}=\underline{A} \boldsymbol{x}$ is completely determined and a simple inversion of $\underline{A}$ would return $\hat{x}=\underline{A}^{-1} \boldsymbol{y}=\boldsymbol{x}_{0}$ perfectly. This 
dependency on $M$ is implicitly stated in the RIP property results above - a sensing matrix $\underline{A}$ only holds the property if the number of its rows obeys $M>\varphi(k ; N)$ [51], where $\varphi$ is some function dependent on $k$ and $N$. An important part of the Compressed Sensing literature focuses on obtaining exact values for the minimum size $M^{*}$ necessary for perfect reconstruction (e.g. [55, 132]).

For now, as an example, consider Figure 1.4 where the problem $\left(P_{1}\right)(1.7)$ was solved for two different values of $M$. Note that if $M$ is too small (Figure 1.4a), reconstruction is imperfect; but if $M$ is above a certain threshold (Figure 1.4b), then the reconstruction error $(1 / N)\left\|\hat{\boldsymbol{x}}-\boldsymbol{x}_{0}\right\|_{2}^{2}$ vanishes.

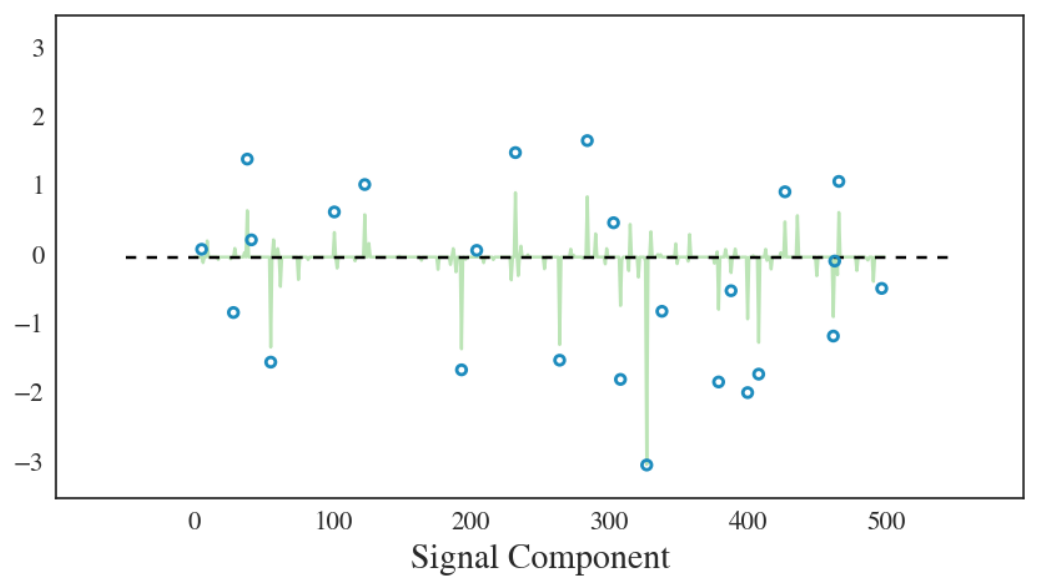

(a) Example of too small $M(M=75)$. Perfect reconstruction is impossible with $\left(P_{1}\right)$. The reconstructed signal (green) does not approximate well the original signal (blue).

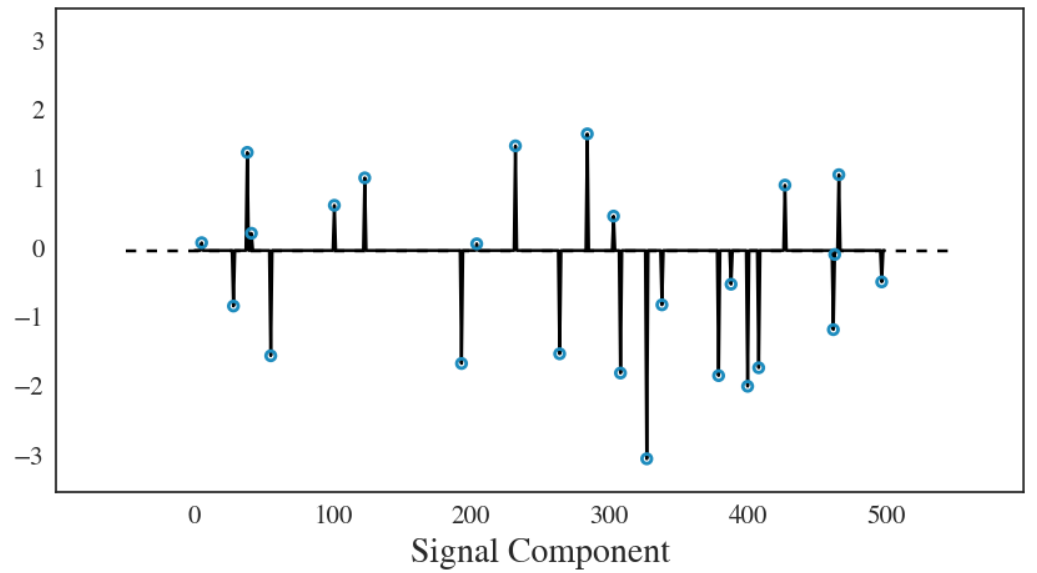

(b) Example of a sufficiently large $M(M=125)$, for which perfect reconstruction (black) becomes possible with $\left(P_{1}\right)$.

Figure 1.4: Effect of the number $M$ of noiseless measurements on the reconstruction accuracy of a sparse signal using the $\ell_{1}$-minimization scheme for the noiseless standard Compressed Sensing problem. In these examples, $N=500$ and $k=25$ (5\%). The two cases above correspond to $M / N=15 \%$ and $M / N=25 \%$, respectively. 
To better understand the statistical properties of the $\ell_{1}$ reconstruction in the Compressed Sensing setting, 100 independent simulations of the entire process (signal $\boldsymbol{x}_{0}$ generation, measurement matrix $\underline{A}$ construction and solution of $\left.\left(P_{1}\right)\right)$ were made for each pair $(\alpha, \rho)$, where $\alpha:=M / N$ is the measurement rate and $\rho:=k / N$ is called the sparsity of the signal. The average of the normalized reconstruction errors

$$
\mathrm{e}:=\frac{1}{N} \frac{\left\|\hat{\boldsymbol{x}}-\boldsymbol{x}_{0}\right\|_{2}^{2}}{\left\|\boldsymbol{x}_{0}\right\|_{2}^{2}}
$$

resulting from these simulations were plotted in a phase diagram (Figure 1.5). Note that in the upper-left region, the error is null, while in the bottom-right of the diagram reconstruction is far from perfect. These regions seem to be well separated — in Chapter 2, we will introduce the replica method, a Statistical Physics technique that allows an accurate description of such phase transitions in certain limits $(N \rightarrow \infty$ with $\rho \equiv k / N$ kept constant).

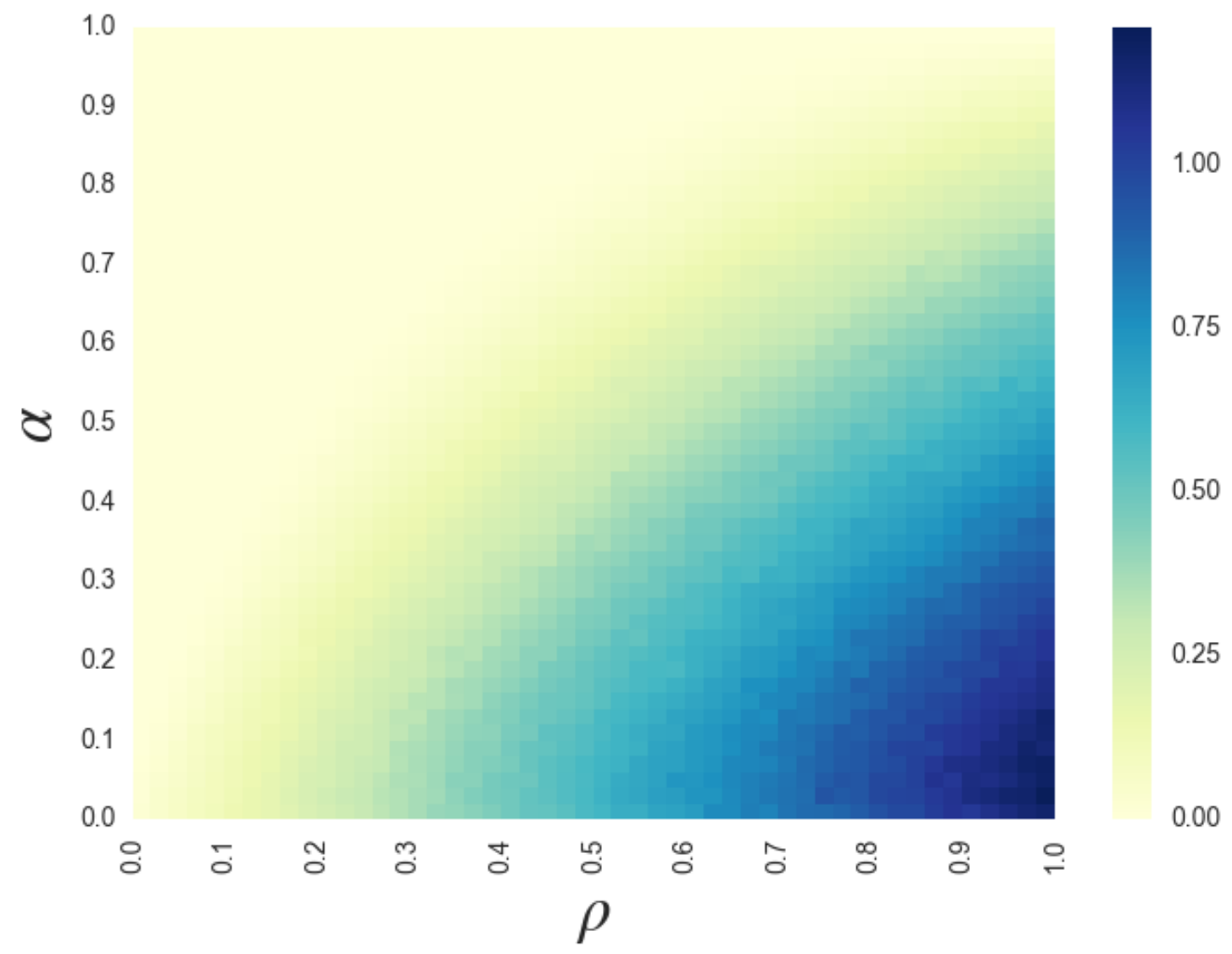

Figure 1.5: Phase diagram of the $\left(P_{1}\right)$ or Basis Pursuit problem (1.7) for the noiseless standard Compressed Sensing problem. Each pair $(\alpha, \rho)$ corresponds to the average of 100 simulations of the SPGL1 implementation [124, 125] with $N=500$. The color represents the normalized reconstruction error $\mathrm{e}=(1 / N)\left\|\hat{\boldsymbol{x}}-\boldsymbol{x}_{0}\right\|_{2}^{2} /\left\|\boldsymbol{x}_{0}\right\|^{2}$. Note that for sufficiently large $\alpha$ (i.e. sufficiently large $M)$ the error vanishes for all $\rho$. 
For last, let us consider the noisy Standard CS setting. As mentioned above, the common approach in Compressed Sensing consists in solving the LASSO problem (1.10). But before any thorough analysis of its results, Figure 1.6 demonstrates the result of a different minimization, the Ridge Regression/Tikhonov Regularization $\min _{\boldsymbol{x}}\|\boldsymbol{y}-\underline{A} \boldsymbol{x}\|_{2}^{2}+\lambda\|\boldsymbol{x}\|_{2}^{2}$. Despite its strong similarity to the LASSO, the $\ell_{2}$ regularization term is not sparsity-promoting - generally, its effect is one of splitting the error among all components, which in turn have their values reduced [57]. ${ }^{11}$

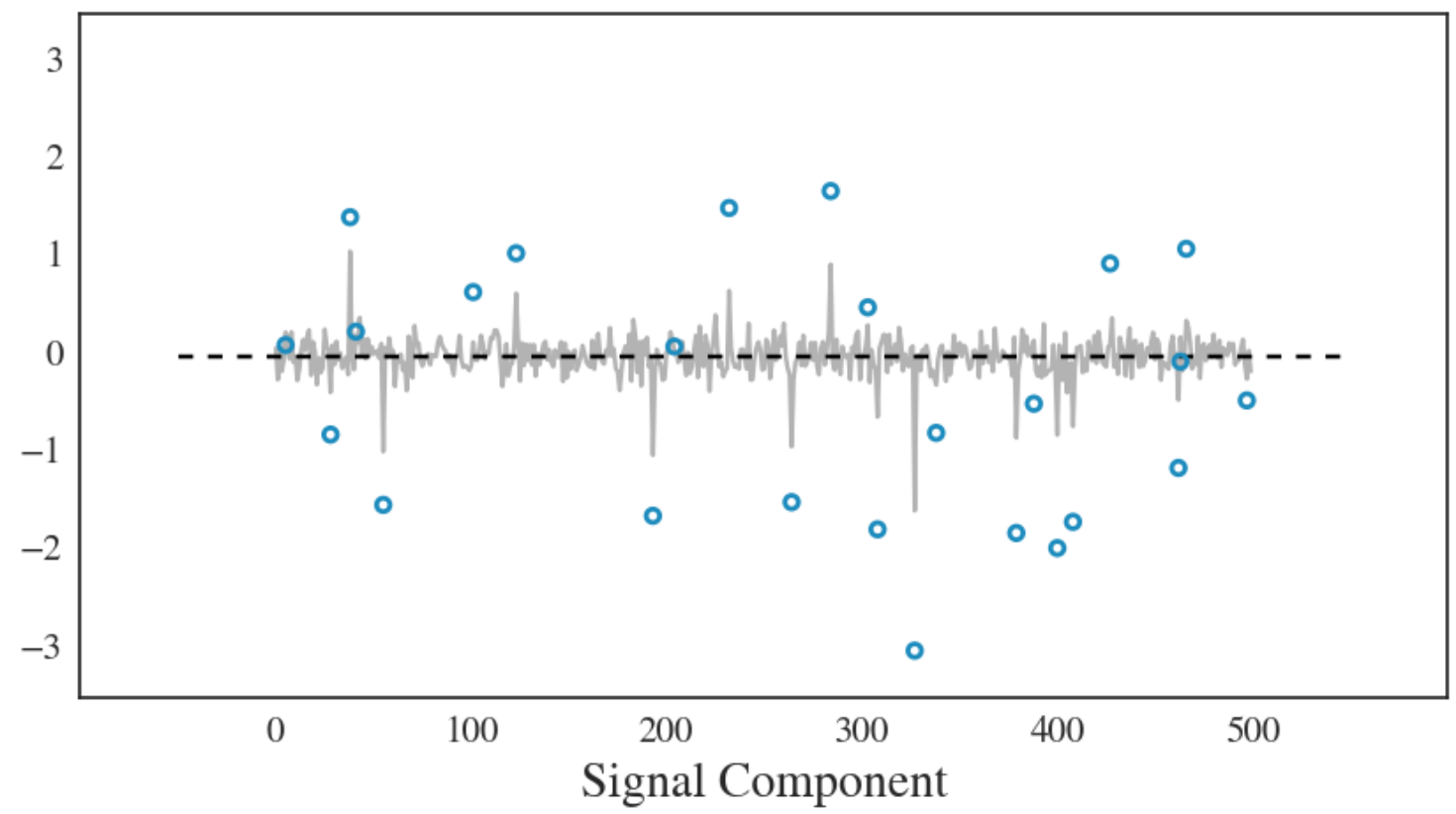

Figure 1.6: An example of the $\ell_{2}$-minimization problem for the noiseless standard Compressed Sensing problem. Here, $N=500, k=25, M=125$ and the original signal is shown in blue. While $M$ is large enough for the $\left(P_{1}\right)$ reconstruction to work perfectly (Figure $1.4 b)$, the solution of the $\left(P_{2}\right)$ minimization shown here in gray is another example of how the $\ell_{2}$-norm splits the error among all components and drives all of them to small values.

As for the solutions to the LASSO, Figure 1.7 compares reconstruction examples for diverse values of $\lambda$ in (1.10), in a setting of low signal-to-noise ratio. The number of measurements was chosen carefully to allow good reconstruction accuracy. When $\lambda$ is too large (Figure 1.7a), sparsity is heavily enforced, resulting in more zero components than ideal; note that even the non-zero components are typically smaller than they should. When $\lambda$ is too small (Figure 1.7b), the reconstructed vector $\hat{\boldsymbol{x}}$ is not sparse enough - small components sneak in, even though the non-zero components appear to be well reconstructed. In the limit $\lambda \rightarrow 0$ (not shown here) it is clear that the regularization term would have no effect at all. In Figure 1.7c, the

\footnotetext{
${ }^{11}$ In addition, it can be shown [92] that in the presence of irrelevant parameters, while the number of examples necessary to learn a concept with $\ell_{1}$-regularization grows logarithmically, with $\ell_{2}$-regularization the number grows at least linearly in the worst-case scenario.
} 
ideal value for $\lambda$ was found through cross-validation.

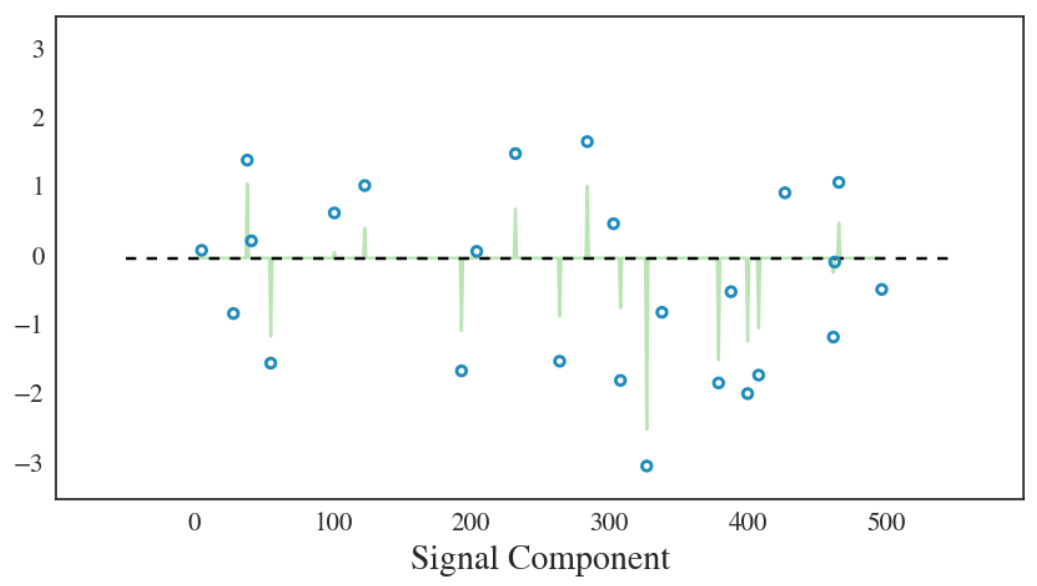

(a) $\lambda \simeq 40 \times \lambda^{*}$

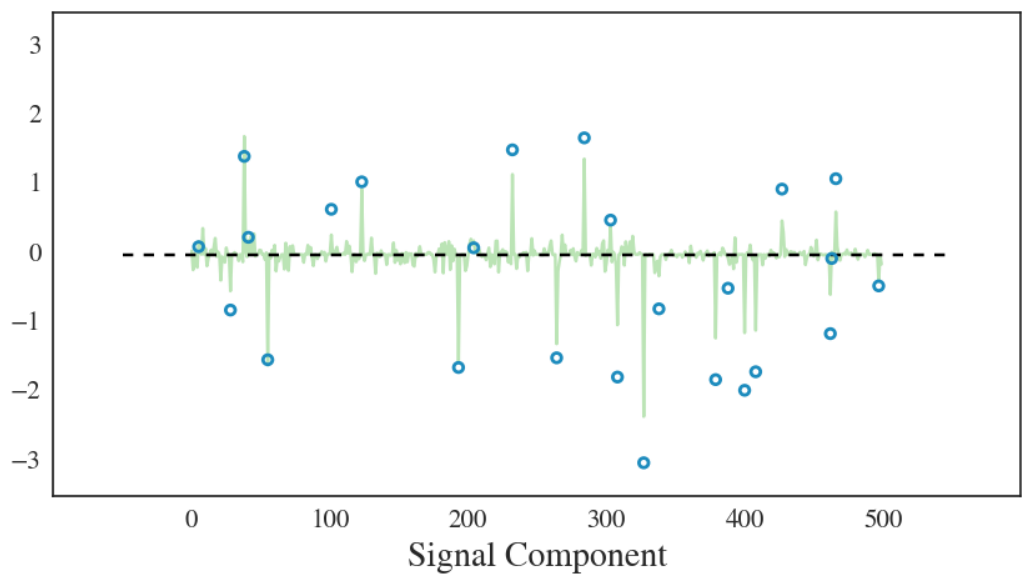

(b) $\lambda \simeq \lambda^{*} / 40$

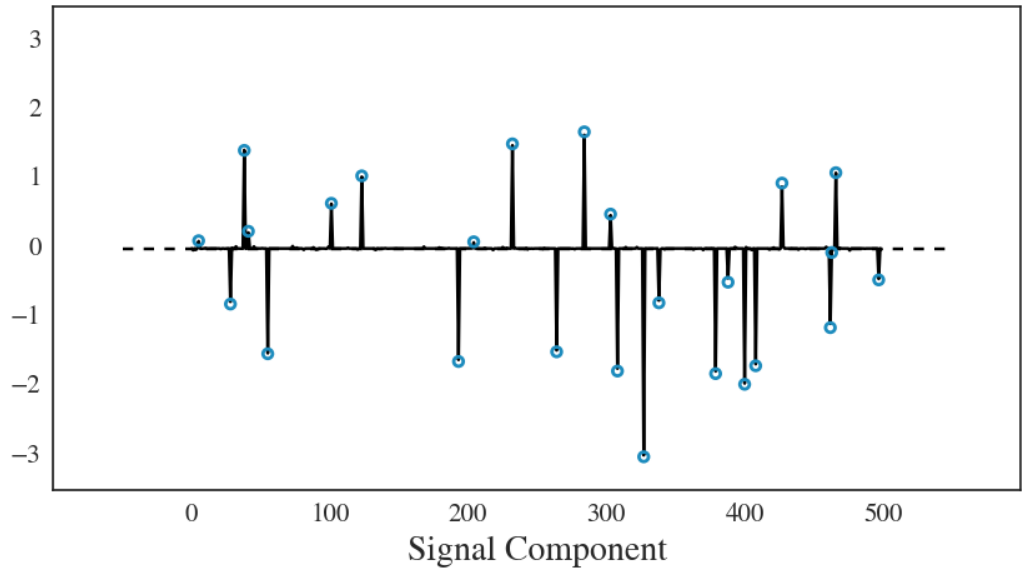

(c) $\lambda=\lambda^{*}$

Figure 1.7: Comparison of the reconstruction accuracy for different values of $\lambda$ in the LASSO (1.10). Shown here are simulations of the noisy standard Compressed Sensing setting, with $N=500, M=250$ and $\sigma_{n}^{2}=10^{-4}$. The optimal value $\lambda^{*} \simeq 2.5 \times 10^{-5}$ was found through cross-validation. Note how too large $\lambda$ means variables are forced to zero, while for small $\lambda$ the regularizing effect is visibly small. 


\subsection{CS in ACtion (2): Single-PIXel CAMERA}

Perhaps the practical application of easiest visualization of the Compressed Sensing ideas is the single-pixel camera developed by researchers from the Department of Electrical and Computer Engineering at Rice University [133, 42, 117]. The hardware (Figures 1.8a and 1.8b) is remarkably simple: light from a scene goes through a biconvex lens and an image is formed on an array of $L_{1} \times L_{2}=N$ movable tiny mirrors over a plate (i.e. a Texas Instruments' Digital Micro-mirror Device). Each of these mirrors can be rotated about a hinge and positioned in either of two possible positions: $+12 /-12$ degrees relative to the plate, which in their architecture corresponds to pointing to or away from a second lens. Any reflected light that shines on this second lens is then focused by it into a single photo-diode, yielding some integrated voltage count $v$.

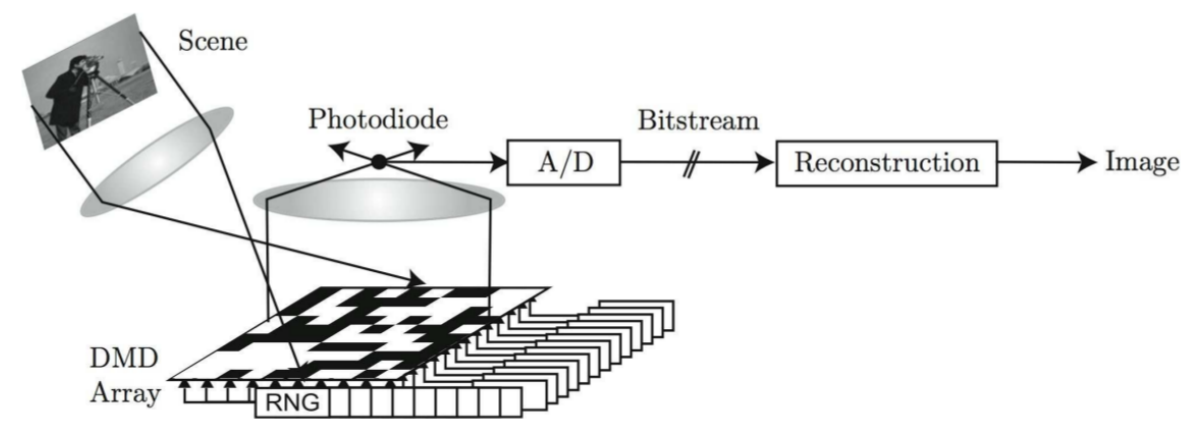

(a) Schematics

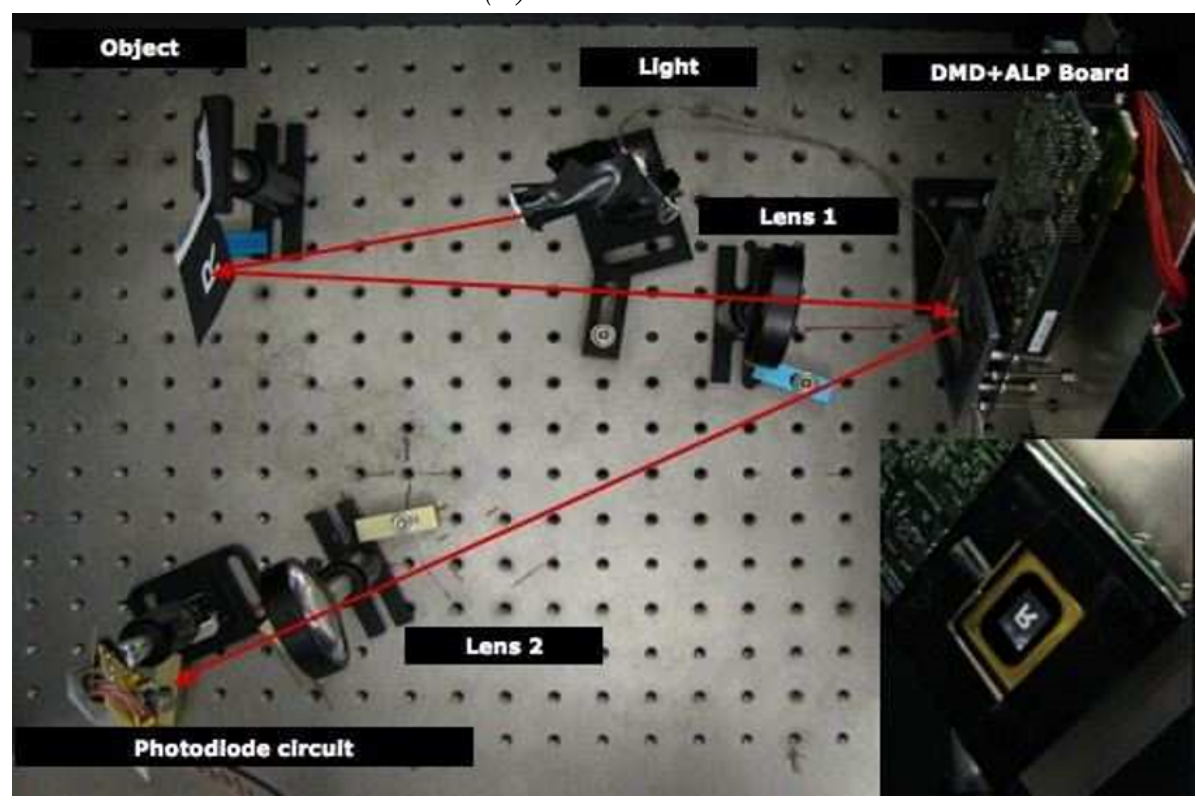

(b) Actual hardware

Figure 1.8: Single-pixel camera hardware, taken from [133] 
Any configuration $m$ of all mirrors' positions correspond to a certain amount of light reaching the photodiode, and therefore to a specific voltage $v(m)$ as well. We can assume that mirrors pointing away from the second lens do not contribute to $v(m)$, and mirrors pointing towards the lens contribute perfectly, reflecting all light that reaches it. This means we can write the expression for $v(m)$ as

$$
v(m) \propto \mathbf{A}(m) \cdot \boldsymbol{x}_{0}
$$

where $\boldsymbol{x}_{0} \in \mathbb{R}^{N}$ is the flattened scene and $\mathbf{A}(m)$ is a vector of ones where the mirrors are placed in the +12 position and zero otherwise. The number $N$ of mirrors will be the final number of reconstructed pixels and therefore the resolution of the final digital image. By randomly generating a number $M$ of different mirror configurations $m_{i}, i=1,2, \ldots, M$, one can easily see how Compressed Sensing comes into the picture.

Figure 1.9 shows some of the photographs obtained with this hardware. By shining light from a LED source over a printout of the letter $R$ (Figure 1.9a), an image (Figure 1.9b) is formed at the mirrors/DMD plane after going through the first lens. For each $m_{i}$ configuration, random parts of this image are reflected by the mirrors and their light integrated in the photo-diode. All results $v\left(m_{i}\right)$ are then stored together with the mirror configurations $\mathbf{A}\left(m_{i}\right)$ which generated them, so that a reconstruction algorithm can then be employed to recover the original scene (the letter $R$, in this case). The authors used a tree matching pursuit algorithm [44, 76] for the recovery and Figures $1.9 \mathrm{c}$ and $1.9 \mathrm{~d}$ show the reconstruction for $M=819$ and $M=1600$, respectively. ${ }^{12}$

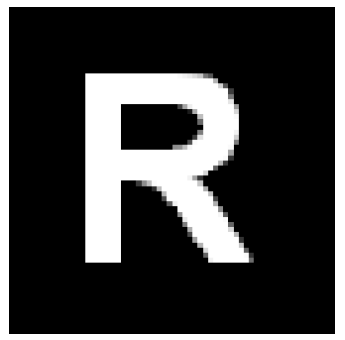

(a) Real scene

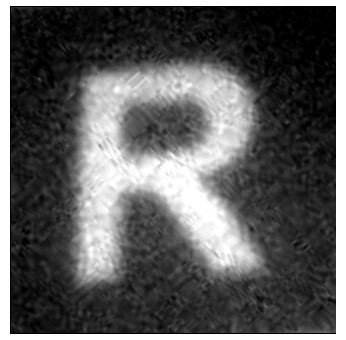

(b) Image at $D M D$

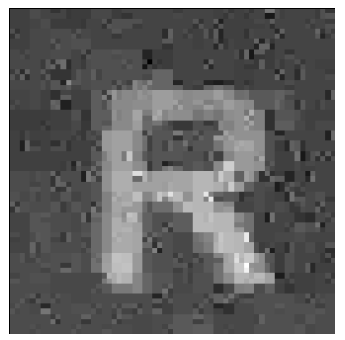

(c) $M=819$

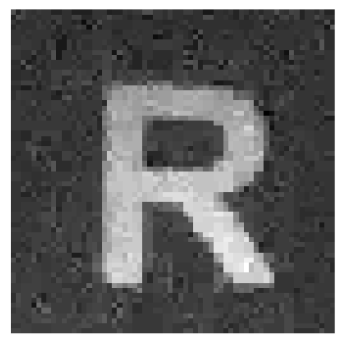

(d) $M=1600$

Figure 1.9: Single-pixel imaging made possible through Compressed Sensing techniques (results adapted from [133]). Shown here are real results obtained with the use of the hardware described above.

\footnotetext{
${ }^{12}$ The DMD can be arranged in blocks, so that groups of neighboring mirrors point in the same direction, thus limiting the "number of pixels" present in the final image. For these experiments, the authors used only a $768 \times 768$ square section from the original $1024 \times 768$ DMD and further divided the visible part into $64 \times 64=4096$ square groups. Thus, the values of $M$ shown above correspond to $\alpha \simeq 2$ and $\alpha \simeq 4$, respectively.
} 
One important thing to note is that $\boldsymbol{x}_{0}$ is not necessarily sparse as it comes it just needs to be sparse in some basis. As we have seen before, images tend to be sparse (or at least compressible) in the Haar basis, so it is possible to write

$$
\boldsymbol{x}_{0}=\underline{H} \boldsymbol{\theta}_{0},
$$

where $\boldsymbol{\theta}_{0}$ is the Haar expansion of $\boldsymbol{x}_{0}$ and $\underline{H}$ is the Haar matrix. What the authors have done in their work [42] was to rewrite the LASSO in the following form:

$$
\hat{\boldsymbol{\theta}}=\underset{\boldsymbol{\theta}}{\arg \min }\|\boldsymbol{\theta}\|_{1} \quad \text { s.t. } \quad \boldsymbol{v}=\underline{A H} \boldsymbol{\theta},
$$

where $\underline{A}$ is a matrix with $\mathbf{A}\left(m_{i}\right)$ as its rows and $\boldsymbol{v}=\left(v_{i}\right)$ is a vector with all photodiode counts. In this setup, what is been recovered is not the original scene per $s e$, but actually its Haar expansion (which is sparse). A final estimate can then be obtained by $\hat{\boldsymbol{x}}=\underline{H} \hat{\boldsymbol{\theta}}$. Figure 1.10 shows a reproduction of this process through computer simulations. ${ }^{13}$

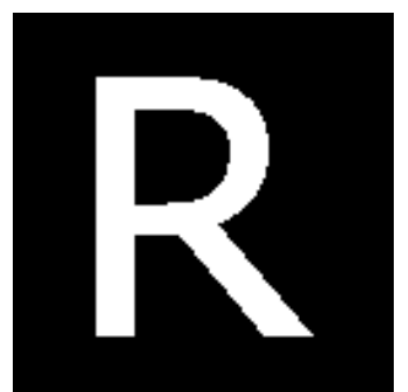

(a) Original

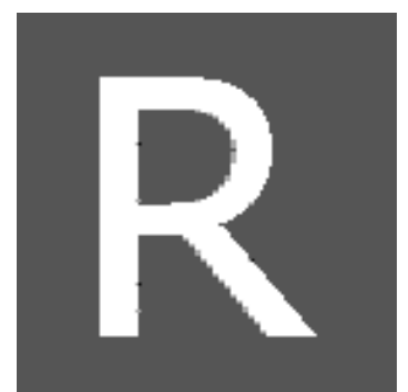

(b) Compressed

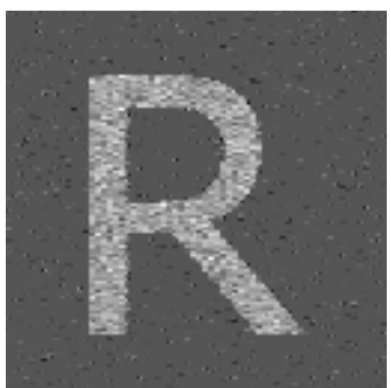

(c) Reconstructed

Figure 1.10: Single-pixel camera simulation. All images are $128 \times 128$ pixels. (a) The original image. (b) Compressed image made of the 1600 ( 10\%) largest Haar expansion coefficients. (c) Image reconstructed from 6400 measurements $(\alpha \simeq 0.4)$ through solution of the LASSO scheme (1.18).

This imaging architecture presents a number of features which make it interesting not only from a theoretical point of view, but also as a practical imaging system [133]: (a) Since there is a single photon detector in the camera, it is possible to use very sensitive sensors (which would be prohibitively expensive in a full CCD or CMOS). Thus it might be possible to explore wavelengths that nowadays are not economically viable. (b) The camera is progressive, in the sense that more measure-

\footnotetext{
${ }^{13}$ In the simulations (as well as in the original work), all measurements configurations $\mathbf{A}(m)$ consisting of $0 / 1$ factors were replaced by Rademacher configurations $-1 / 1$. By doing that one must also compensate the measurement values $v(m)$ with $v(m) \rightarrow 2 v(m)-v(1)$, where the first configuration is obtained with all mirrors in the +12 position, i.e. $v(1)=\sum_{i} x_{i}^{0}$.
} 
ments result in better reconstruction. In fact, since every measurement contains the same amount of information [31], the camera is robust in the sense that some of the measurements can be lost without much loss in the final result. (c) Measurements resemble noise in absence of the knowledge of all $\mathbf{A}\left(m_{i}\right)$ (i.e. knowledge of the seed that generated all configurations in the pseudo-random number generator). By consequence, the reconstructed image is encrypted if the seed is stored safely. These points demonstrate that Compressed Sensing can be more useful than just reducing the number of measurements necessary for the signal recovery.

The experimental results in this section (and also the theoretical results in this Chapter as a whole) show the viability of Compressed Sensing as a new signal acquisition paradigm. In the next chapter the Bayesian recovery scheme will be introduced, along with Statistical Physics tools to analyze the typical performance of reconstruction methods. 

Chapter 2

\section{The BAyesian APPROACH AND Statistical Physics}

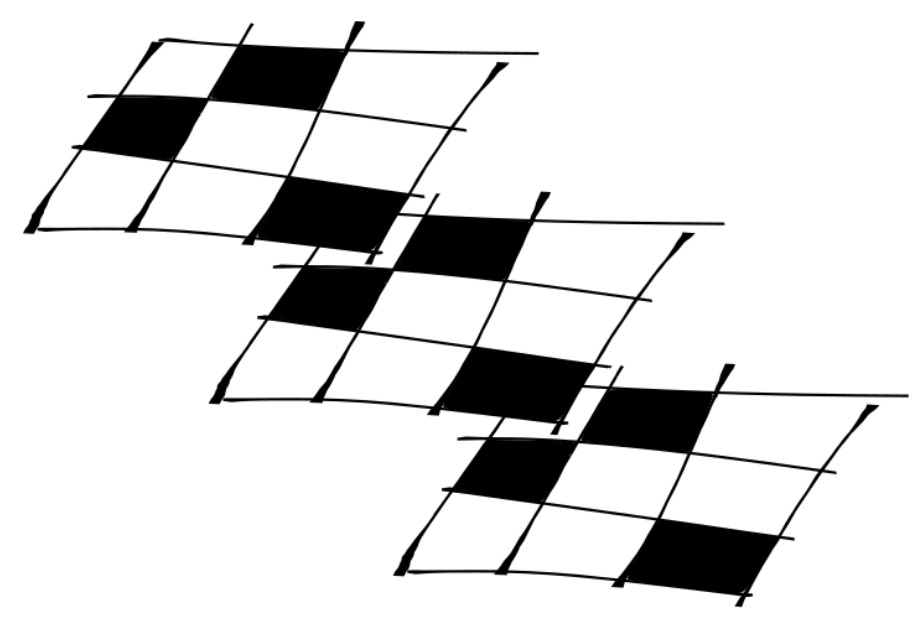




\subsection{INTRODUCTION}

The pedigree of the Bayesian method as a valid inference tool is well established. In the 18th century, Rev. Thomas Bayes [9] tackled the problem articulated by Bernoulli [12] of how one could use deductive logic to shed light on the inductive logic required for everyday life (which is full of situations where one cannot argue with certainty) [83]. More recently, it has been successfully applied to areas as diverse as time-series analysis [1, 73], neural networks [103, 91], genetics [65, 104, 138, 66], crystallography [53], and decision theory [10], among others. To our interest here, it is noteworthy the introduction of Bayesian reconstruction methods for sparse signal recovery in the Compressed Sensing literature [122, 75]. In this chapter we will describe the Bayesian scheme and analyze it from the point of view of Statistical Physics.

Bayes theorem, ${ }^{1}$ which can be famously derived (for an example, [68]) from Cox's quantitative rules necessary for consistent reasoning and logic in what is called the Cox's theorem [28, 29], says that

$$
P(A \mid B)=\frac{P(B \mid A) P(A)}{P(B)}
$$

for any propositions $A$ and $B$. Here, $P(A)$ denotes the probability that proposition $A$ is true and $P(A \mid B)$ is a conditional probability that should be understood as "the probability of proposition $A$ being true taken into account that proposition $B$ is true." ${ }^{2}$ A proposition might be something like

$$
\text { A } \equiv \text { variable } X \text { has the value } X^{\prime}
$$

and in this case one simply writes $P\left(X=X^{\prime} \mid B\right)=P\left(X^{\prime} \mid B\right)$.

All elements in (2.1) have standard names and meanings. On the L.H.S. we have the posterior distribution, which usually comprises our inference result. $P(A)$ is called the prior distribution. It embodies all knowledge about the experiment that does not come directly from the data. The other factor on the numerator on the R.H.S., $P(B \mid A)$, is the likelihood - it is here that we encode any information about the experiment. The denominator, $P(B)$, is called the evidence. Since it corresponds to the normalization of the posterior distribution, it is often ignored so that Bayes'

\footnotetext{
${ }^{1}$ The present form of Bayes' theorem is due to Laplace [82] who besides rediscovering its contents also used it to solve problems in celestial mechanics, medical statistics and jurisprudence [115].

${ }^{2} P(A \mid B)$ is usually read as "probability of $A$ given $B$ "
} 
theorem becomes $P(A \mid B) \propto P(B \mid A) P(A)$. In the CS setting, the prior distribution might contain information about specific characteristics of the signal, such as how it is generated, and the likelihood codes information about how measurements are obtained (such as the fact that they are obtained from linear projections $\boldsymbol{A} \cdot \boldsymbol{x}$ ) along with information about presence or absence of noise, for example. That is, the likelihood corresponds to the channel $P(y \mid u)$ of expression (1.2). In particular, the Bayes' theorem for CS can be stated as

$$
\overbrace{P(\boldsymbol{x} \mid \underline{A}, \boldsymbol{y})}^{I}=\frac{P(\boldsymbol{y} \mid \underline{A}, \boldsymbol{x}) P(\boldsymbol{x} \mid \underline{A})}{P(\boldsymbol{y} \mid \underline{A})}=\overbrace{\frac{P(\boldsymbol{y} \mid \underline{A} \boldsymbol{x})}{P(\boldsymbol{y} \mid \underline{A})}}^{I I} \overbrace{P(\boldsymbol{x})}^{I I I} .
$$

The last equality comes from the fact that the prior knowledge of the signal (expressed numerically in $P(\boldsymbol{x})$ ) does not depend on $\underline{A}$ in any manner. A possible way to read this expression is: considering that we have got in hand all measurement results $\boldsymbol{y}$ (II) and given all we know about how the signal $\boldsymbol{x}$ was generated (III), what is our best hunch about the actual values of $\boldsymbol{x}(\mathrm{I})$ ?

Commonly, in theoretical studies, the signal components $x_{0, i}, i=1, \ldots, N$, are considered to be uncorrelated $^{3}$ and the predominant sparse-inducing generative model for the signal is described as

$$
\phi_{0}(\boldsymbol{x})=\prod_{i=1}^{N}\left[(1-\rho) \delta\left(x_{i}\right)+\rho g\left(x_{i}\right)\right]
$$

where $g\left(x_{i}\right)$ is a function with no finite mass at $x_{i}=0$ (i.e. all probability that $x_{i}$ is exactly zero is given by the delta factor). Tipically — but not necessarily -,$g(x)$ takes the form of a normal distribution $\mathcal{N}\left(\bar{x}, \sigma^{2}\right)=\left(\sqrt{2 \pi \sigma^{2}}\right)^{-1} \exp \left[-(x-\bar{x})^{2} / 2 \sigma^{2}\right]$, and (2.4) is then called a Gauss-Bernoulli distribution. Note the implication of sparsity already in the signal model: since each component will be zero with probability $1-\rho$, in average only a fraction $\rho=k / N$ of the signal's components will be non-zero. In the controlled and ideal scenario where one knows exactly the generative distribution of the signal, the prior in (2.3) can be equated to this distribution:

$$
P(\boldsymbol{x})=\phi_{0}(\boldsymbol{x})
$$

As mentioned above, a particularly popular noiseless setting is the one with measurements of the kind $\boldsymbol{y}=\underline{A} \boldsymbol{x}$; in the presence of additive Gaussian noise they become $\boldsymbol{y}=\underline{A} \boldsymbol{x}+\boldsymbol{\xi}$, where each $\left(\xi_{i}\right)_{i=1}^{N}$ is a Gaussian random variable with zero

\footnotetext{
${ }^{3}$ The argument being that correlations could be explored if present and reconstruction would then become easier. Why then go for easier targets?
} 
mean and variance $\sigma_{n}^{2}$. These standard $C S$ models can be rewritten as probability distributions for $\boldsymbol{y}$ (or likelihoods) in the following way:

$$
\begin{cases}\text { (noiseless): } & P(\boldsymbol{y} \mid \underline{A}, \boldsymbol{x})=\prod_{\mu=1}^{M} \delta\left(y^{\mu}-\sum_{i=1}^{N} A_{i}^{\mu} x_{i}\right) \\ \text { (noisy): } & P(\boldsymbol{y} \mid \underline{A}, \boldsymbol{x})=\prod_{\mu=1}^{M} \frac{1}{\sqrt{2 \pi \sigma_{n}^{2}}} \exp \left[-\left(1 / 2 \sigma_{n}^{2}\right)\left(y^{\mu}-\sum_{i=1}^{N} A_{i}^{\mu} x_{i}\right)^{2}\right] .\end{cases}
$$

It is easy to notice that even though the distributions above are presented separately as two different cases, the distribution for noiseless measurements can also be obtained from the noisy measurement distribution in the limit $\sigma_{n} \rightarrow 0$.

With equations (2.6) and (2.4), Bayes' theorem (2.1) becomes

$$
P(\boldsymbol{x} \mid \underline{A}, \boldsymbol{y}) \propto \prod_{i=1}^{N}\left[(1-\rho) \delta\left(x_{i}\right)+\rho g\left(x_{i}\right)\right] \prod_{\mu=1}^{M} \frac{1}{\sqrt{2 \pi \sigma_{n}^{2}}} \mathrm{e}^{-\left(1 / 2 \sigma_{n}^{2}\right)\left(y_{\mu}-\sum_{i=1}^{N} A_{i}^{\mu} x_{i}\right)^{2}}
$$

where, once again, one should consider $\sigma_{n} \rightarrow 0$ in the absence of noise. The posterior distribution on the L.H.S. of (2.7) then is all that we can say about the signal given the information available. But for most cases, it is of interest to have a simple estimate of the signal, rather than a full distribution. Different estimators are possible. A common choice is the Maximum-A'posteriori-Probability (MAP) estimator, which consists in the vector which maximizes the posterior distribution. In other words, the signal estimate would then be obtained from the posterior by $\hat{\boldsymbol{x}}^{\mathrm{MAP}}:=\max _{x} P(\boldsymbol{x} \mid \underline{A}, \boldsymbol{y}) .{ }^{4}$ In this work, though, when we talk about a Bayesian estimate we will be referring to

$$
\boldsymbol{m} \equiv \hat{\boldsymbol{x}}^{\mathrm{mmse}}:=\int \mathrm{d} \boldsymbol{x} \boldsymbol{x} P(\boldsymbol{x} \mid \underline{A}, \boldsymbol{y}),
$$

commonly known as the Minimum-Mean-Squared-Error (mmse) estimator. By definition, this estimator minimizes the mean-squared error mse, defined as

$$
\operatorname{mse}(\boldsymbol{z})=\int d \boldsymbol{x} P(\boldsymbol{x} \mid \underline{A}, \boldsymbol{y})\|\boldsymbol{z}-\boldsymbol{x}\|_{2}^{2}
$$

for any vector $\boldsymbol{z}$. If one takes the derivative with respect to $\boldsymbol{z}$,

$$
\frac{\partial \mathrm{mse}(\boldsymbol{z})}{\partial \boldsymbol{z}}=2 \int d \boldsymbol{x} P(\boldsymbol{x} \mid \underline{A}, \boldsymbol{y})(\boldsymbol{z}-\boldsymbol{x})=2 \boldsymbol{z}-2 \int d \boldsymbol{x} P(\boldsymbol{x} \mid \underline{A}, \boldsymbol{y}) \boldsymbol{x}
$$

and equates the result to zero, then it is trivial to see that the minimum of mse is indeed expression (2.8).

But how to efficiently calculate $\boldsymbol{m}$ ? Let us define the marginal posterior proba-

\footnotetext{
${ }^{4}$ For constant/flat priors $P(\boldsymbol{x} \mid \boldsymbol{A})=c$, this is the same as the well-known Maximum-Likelihood estimator, which maximizes the likelihood.
} 
bility distribution for component $x_{i}$ as

$$
P_{i}\left(x_{i}\right)=\int \prod_{j \neq i} d x_{j} P(\boldsymbol{x} \mid \underline{A}, \boldsymbol{y}) .
$$

With it, each element of the mmse estimator might be expressed as

$$
m_{i}=\int d x_{i} x_{i} P_{i}\left(x_{i}\right)
$$

In theory then, once the marginals are available, $\boldsymbol{m}$ is directly obtainable. But the practical difficulty lies in the exact computation of the marginals $P_{i}\left(x_{i}\right)$, since it requires exponential time [90], becoming unfeasible for large signals. For this reason, works in the literature that follow a Bayesian approach have relied on methods to approximate the marginals and expectations. In particular, graphical modelling theory [4] provides a very efficient method known as message passing [84], from which Compressed Sensing specific implementations have been devised [38, 36, 87, $6,8,100]$. In Appendix A we show a derivation of the implementation presented in $[74,75]$ and known as Approximate Message Passing (AMP).

Figure 2.1 demonstrates examples of Bayesian signal reconstructions with the AMP algorithm for the noiseless standard Compressed Sensing setting. The error curves on the top right panel shows how different values of the measurement rate $\alpha$ influence the reconstruction error also in the Bayesian scenario. For the three values of $\rho$ shown there is a value $\alpha_{c}$ for which every reconstruction with $\alpha>\alpha_{c}$ is perfect (next section will put this analysis on more firm theoretical grounds). The other three panels show the difference in reconstruction accuracy for the same signal $\boldsymbol{x}_{0}$ but different values of $\alpha$ for $\rho=0.2$. 


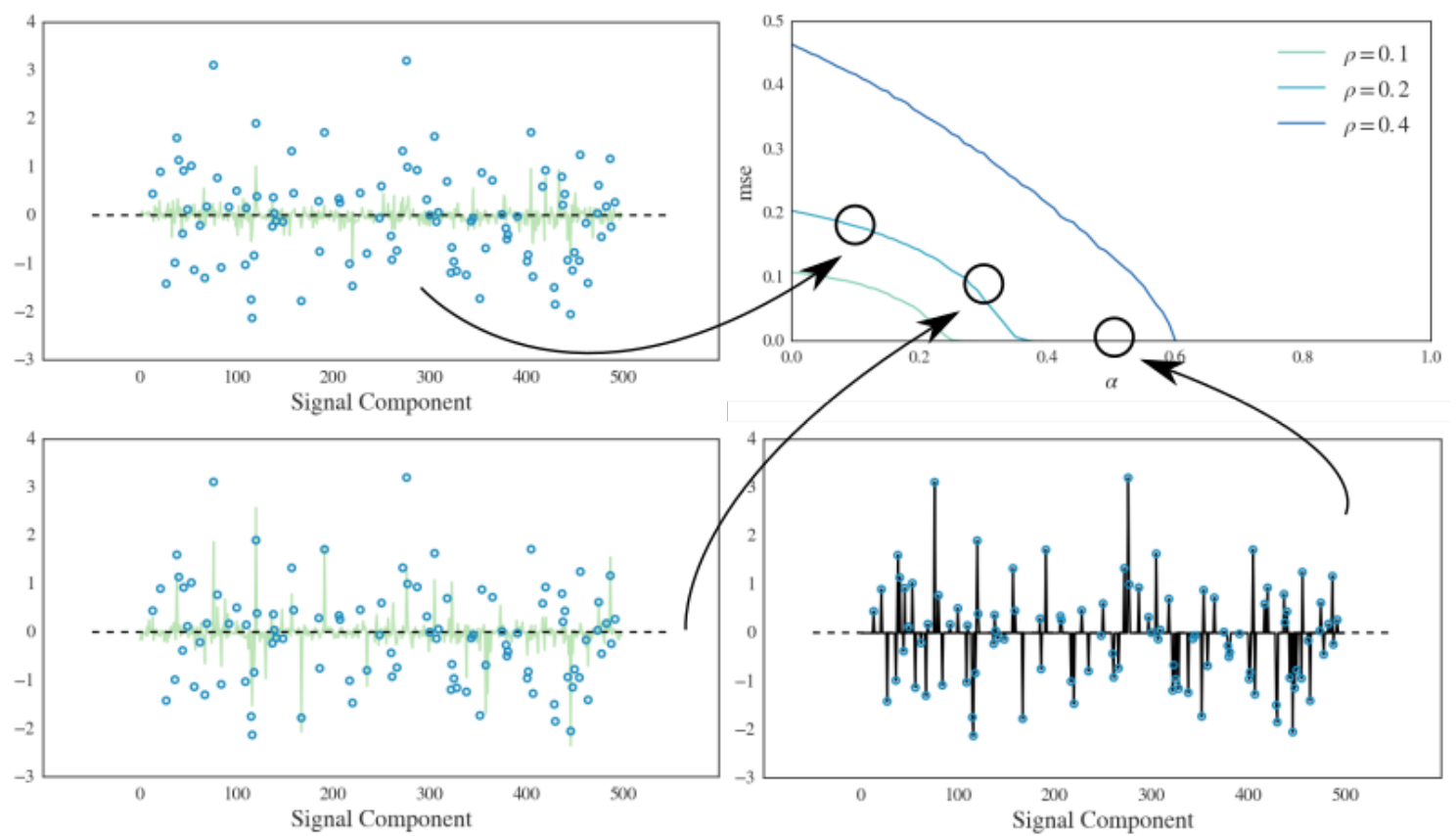

Figure 2.1: Bayesian Compressed Sensing reconstruction with the AMP algorithm (Appendix A). The curves in the top right panel correspond to the average normalized reconstruction error of 100 simulations for each value of $\alpha$ of the noiseless standard CS problem for $N=500$ and $\rho=0.1$ (green), $\rho=0.2$ (light blue) and $\rho=0.4$ (blue). Note that for all three values of $\rho$ there is a value $\alpha_{c}$ for which any reconstruction with $\alpha>\alpha_{c}$ has zero error. The other three panels are examples of signal reconstructions for $\rho=0.2$ and $\alpha=0.1$ (top left), $\alpha=0.3$ (bottom left) and $\alpha=0.5$ (bottom right).

\subsection{Statistical Physics And typical PerformanCE}

In this section we try to characterize the conditions for the Bayesian recovery to work with sufficient accuracy. In the literature, two distinct approaches are commonly found [102]. The first consists in deriving necessary and sufficient conditions for the recovery to work, using arguments from information theory (e.g. [2, 55, 131]) or direct analysis of specific algorithms (e.g. [20, 22, 132]). The second approach comes from the Statistical Physics community - the replica method [85]. By drawing parallels between physical systems and the recovery task at hand, this method allows one to derive statistics regarding the typical (in the sense of occurring with overwhelming probability) reconstructed signal in the large system size $N \rightarrow \infty$ when certain system conditions are satisfied (e.g. $k / N$ kept finite). In particular, by measuring the expected overlap between the original signal and its reconstruction, it is possible to obtain the expected reconstruction error of a determined algorithm, finding its limitations or phase transitions in the phase space of the measurement rate $\alpha$ and the sparsity of the signal $\rho$. An example of such a line of argument can be found in [127]. 
In 1975, building up on a technique devised for the study of molecular networks in rubbers [45], Edwards and Anderson [46] introduced the replica method as a way to analyze the free energy of disordered systems [93]. Since then, the method has been used in very diverse settings, such as superconductivity problems [16], large dual antenna systems [88], combinatorial optimization [84], perceptron learning [52], and error-correcting codes [127]. Last but not least, it already has an established (albeit short) history in Compressed Sensing reconstruction analysis that goes back to 2009-2010 when two papers [70, 58] appeared analyzing the typical reconstruction error of the $\ell_{p}$-reconstruction problems in the large system size limit. It was found that the dividing line in the phase space $\alpha \times \rho$ that was obtained through simulations shown in Figure 1.5 does indeed correspond to a phase transition - there is a critical measuring rate $\alpha_{c}^{P_{1}}(\rho)$ given by the solution to

$$
\begin{aligned}
& \alpha=2(1-\rho) \int_{s}^{\infty} \mathcal{D} z(z-s)^{2}+\rho\left(1+s^{2}\right), \\
& \alpha=2(1-\rho) H(s)+\rho,
\end{aligned}
$$

where $\mathcal{D} z=(1 / \sqrt{2 \pi}) \exp \left(-z^{2} / 2\right)$ is the standard Gaussian measure and $H(x)=$ $\int_{x}^{\infty} \mathcal{D} z$. For $\alpha>\alpha_{c}^{P_{1}}(\rho)$, the signal can be reconstructed perfectly; for $\alpha<\alpha_{c}^{P_{1}}(\rho)$ the mse diverges, indicating an impossible reconstruction regime. The problems $\left(P_{0}\right)$ and $\left(P_{2}\right)$ exhibit a much simpler behaviour, with $\alpha_{c}^{P_{0}}(\rho)=\rho$ and $\alpha_{c}^{P_{2}}(\rho)=1$.

\section{The Replica Method}

Consider a Statistical Physics ensemble of many-particle systems, each one with microscopic state, described by the $N$-dimensional vector $\boldsymbol{x}$, which occurs with probability $p(\boldsymbol{x})$. To each state is also associated an energy $H(\boldsymbol{x})$, so that at thermal equilibrium the ensemble energy $E=\int d \boldsymbol{x} p(\boldsymbol{x}) H(\boldsymbol{x})$ is kept constant. Since from the second law of thermodynamics the entropy $S=\int d \boldsymbol{x} p(\boldsymbol{x}) \log p(\boldsymbol{x})$ must be at its maximum at equilibrium, one can maximize $S$ subject to $E$, producing the Gibbs' distribution $p(\boldsymbol{x})=(1 / Z) \exp [-\beta H(\boldsymbol{x})]$, where $\beta$ is a Lagrange multiplier determined by $E$ and

$$
Z=\int d \boldsymbol{x} \exp [-\beta H(\boldsymbol{x})]
$$

is called the partition function. Key thermodynamical properties of the system can be obtained from $Z$ and/or the free-energy density $f=-(\beta N)^{-1} \log Z$ (which, under the described conditions, should be at its minimum as a function of $p(\boldsymbol{x}))$.

Let us then make the connection with the inference problem by identifying $p(\boldsymbol{x}) \rightarrow P(\boldsymbol{x} \mid \underline{A}, \boldsymbol{y})$. In the Bayesian formulation (2.7) of the standard Compressed 
Sensing problem, $\underline{A}$ and $\boldsymbol{y}$ are random variables, making the partition function (and by consequence the free-energy) a random variable as well. That is, $Z=Z(\underline{A}, \boldsymbol{y})$ is a function of the disorders $\underline{A}$ and $\boldsymbol{y}$. Typically, the distribution of the partition function is broad with long tails even for large system sizes [52], so that its average can be dominated by rare samples [84]. On the other hand, its logarithm is well-behaved, making the average of the free-energy

$$
\bar{f}:=-(\beta N)^{-1}\left\langle\langle\log Z(\underline{A}, \boldsymbol{y})\rangle_{\underline{A}, \boldsymbol{y}},\right.
$$

an interesting starting point to study typical properties in the system. In the expression above, $\left\langle\langle\eta(t)\rangle_{t}\right.$ is the expected value of quantity $\eta(t)$ with respect to the probability distribution of $t$.

But as the dependence of $\log Z$ on $\underline{A}$ and $\boldsymbol{y}$ can be very complicated, direct evaluation of $\langle\langle\log Z\rangle$ is in general prohibitively difficult. Nevertheless, the replica trick $[93,85]$ is a technique to interchange the average and the logarithm in (2.16) in a controlled fashion. By taking the identity

$$
\log x=\lim _{n \rightarrow 0} \frac{d}{d n} x^{n}=\lim _{n \rightarrow 0} \frac{x^{n}-1}{n}
$$

one can substitute $x$ for the partition function $Z$ and take the expected value on both sides, arriving at

$$
\langle\langle\log Z\rangle\rangle=\lim _{n \rightarrow 0} \frac{\left\langle\left\langle Z^{n}\right\rangle-1\right.}{n} .
$$

Although this identity is valid for $n \in \mathbb{R}$, the replica trick consists in calculating $\left\langle\left\langle Z^{n}\right\rangle\right.$ for $n \in \mathbb{N}$ and then after all is done, taking the limit $n \rightarrow 0$. It is not rigorous, it might seem arbitrary, but it turns out that this average is much easier to calculate. And somehow it works in many situations [93].

At this stage, we can interpret $\left\langle\left\langle Z^{n}\right\rangle\right.$ as the partition function of a new replicated system, formed by $n$ replicas each with its own partition function $Z_{i}, i=1, \ldots, n$. Since $\bar{f}$ cannot be written as a simple sum of single replica terms, it is clear that the replicas are not independent of each other and do in fact interact. A correlation (or overlap) matrix $\mathbf{Q}$ for the replicas can be introduced and, as it turns out, $\left\langle\left\langle Z^{n}\right\rangle\right\rangle \sim$ $\int \mu(d \mathbf{Q}) \exp (N g(\mathbf{Q}))$, with $g$ a problem-dependent function and $\mu$ the probability measure of $\mathbf{Q}$. When $N \rightarrow \infty$, the supremum of $\mathbf{Q}$ becomes the only relevant factor in the integral, but this calculation may be cumbersome. As it turns out, since the $n$ replicas are equivalent, in many problems the supremum is identical over all dimensions, i.e. there is replica symmetry, and $\mathbf{Q}$ can be parameterized by only a few order parameters of which the free-energy becomes a function [60]. Finding 
these parameters then is equivalent to describing the system in its equilibrium the extreme of $\bar{f}$ (calculated over the parameterization of $\mathbf{Q}$ ) represents typicality.

\subsection{Replica CAlculation For the Bayesian Re- CONSTRUCTION}

In this section a detailed presentation of all steps described above will be attempted. Although many of these steps are not rigorous nor have been proved mathematically even up to present [84], at the end we present a final comparison between the prescription of the replica method under the replica symmetric assumption for the Bayesian Compressed Sensing problem and results from simulations, where it will be made explicit the agreement of both (and, consequently, the accuracy of the replica method for this scenario).

In the literature there are not many examples of replica calculations performed in detail (noteworthy exceptions are found in $[52,127]$ ). These calculations generally follow one or two templates, where a bag of "tricks" is used in standardized sequence, but the lack of a didactic presentation might make navigation of the works prohibitively difficult, specially for beginners. As such, we expect that this somewhat lengthy section might be of use to a newcomer to the field.

Starting with the Bayesian Compressed Sensing problem (2.3) with any prior $\phi(\boldsymbol{x})$ and generating distribution $\phi_{0}\left(\boldsymbol{x}_{0}\right)$ given by $(2.4)$, the posterior probability distribution for the signal is

$$
P(\boldsymbol{x} \mid \underline{A}, \boldsymbol{y})=\frac{1}{Z(\underline{A}, \boldsymbol{y})} \phi(\boldsymbol{x}) \prod_{\mu=1}^{M} P\left(y^{\mu} \mid \mathbf{A}^{\mu} \cdot \boldsymbol{x}\right)
$$

and its partition function,

$$
Z(\underline{A}, \boldsymbol{y})=\int \mathrm{d} \boldsymbol{x} \phi(\boldsymbol{x}) \prod_{\mu=1}^{M} P\left(y^{\mu} \mid \mathbf{A}^{\mu} \cdot \boldsymbol{x}\right) .
$$

Note that with the exception of the very final steps of the calculation, where $P\left(y^{\mu} \mid \mathbf{A}^{\mu} \cdot \boldsymbol{x}\right)$ will be forced to assume a specific format, all the rest is of sufficient generality for any measurement model $P(y \mid u)$. Our main objective is to calculate $\langle\langle\log Z\rangle$, for which the replica trick (2.18) is useful in indicating that we can calcu-

late instead the $n$-th power of $Z, Z^{n}(\underline{A}, \boldsymbol{y})=\left[\int \mathrm{d} \boldsymbol{x} \phi(\boldsymbol{x}) \prod_{\mu=1}^{M} P\left(y^{\mu} \mid \mathbf{A}^{\mu} \cdot \boldsymbol{x}\right)\right]^{n}$. For general $n$ this might be very complicated, but instead we evaluate this expression 
for integer $n$,

$$
Z^{n}(\underline{A}, \boldsymbol{y})=\int \prod_{a=1}^{n}\left[\mathrm{~d} \boldsymbol{x}_{a} \phi\left(\boldsymbol{x}_{a}\right) \prod_{\mu=1}^{M} P\left(y^{\mu} \mid \mathbf{A}^{\mu} \cdot \boldsymbol{x}_{a}\right)\right]
$$

and only at the end "remember" that $n$ should have been real all the time and take its limit $n \rightarrow 0$. With (2.21), the system is now properly replicated. For now, we would like to evaluate the expected value of the expression above with regards to the disorders $\boldsymbol{y}$ and $\underline{A}$ :

$$
Z^{(n)}:=\left\langle\left\langle Z^{n}(\underline{A}, \boldsymbol{y})\right\rangle\right\rangle_{\underline{A}, \boldsymbol{y}}=\int \prod_{a=1}^{n}\left[\mathrm{~d} \boldsymbol{x}_{a} \phi\left(\boldsymbol{x}_{a}\right)\left\|\left\langle\prod_{\mu=1}^{M} P\left(y^{\mu} \mid \mathbf{A}^{\mu} \cdot \boldsymbol{x}_{a}\right)\right\rangle\right\|_{\underline{A}, \boldsymbol{y}}\right] .
$$

One of the main devices used in replica calculations is the cleverly placed introduction of ones (in multiplications) and zeros (in sums). Here we will multiply the expression above by $1 \equiv \int \mathrm{d} \boldsymbol{x}_{0} \phi_{0}\left(\boldsymbol{x}_{0}\right)$, which does not alter its value:

$$
Z^{(n)}=\int \mathrm{d} \boldsymbol{x}_{0} \phi_{0}\left(\boldsymbol{x}_{0}\right) \prod_{a=1}^{n}\left[\mathrm{~d} \boldsymbol{x}_{a} \phi\left(\boldsymbol{x}_{a}\right) \|\left\langle\prod_{\mu=1}^{M} P\left(y^{\mu} \mid \mathbf{A}^{\mu} \cdot \boldsymbol{x}_{a}\right) \|_{\underline{A}, \boldsymbol{y}}\right]\right.
$$

Let us now define the replica overlap variables

$$
q_{a b}:=\frac{\boldsymbol{x}_{a} \cdot \boldsymbol{x}_{b}}{N}
$$

for $a, b=0,1, \ldots, n$, and call $\mathbf{Q}$ the $(n+1) \times(n+1)$ symmetric overlap matrix $\mathbf{Q}=\left(q_{a b}\right)$. From (2.24), a new set of ones might be obtained as $1=N \int \mathrm{d} q_{a b} \delta\left(\boldsymbol{x}_{a} \cdot \boldsymbol{x}_{b}-N q_{a b}\right)$ for any $a, b$. Any multiplication of these terms is also equal to unity:

$$
1=\prod_{a \geq b} N \int \mathrm{d} q_{a b} \delta\left(\boldsymbol{x}_{a} \cdot \boldsymbol{x}_{b}-N q_{a b}\right)=\int d \mathbf{Q} \prod_{a \geq b} \delta\left(\boldsymbol{x}_{a} \cdot \boldsymbol{x}_{b}-N q_{a b}\right),
$$

where $\mathrm{d} \mathbf{Q} \equiv \prod_{a \geq b} N \mathrm{~d} q_{a b}$. Insertion of this identity in (2.23) results in

$$
\begin{aligned}
Z^{(n)}= & \int d \boldsymbol{Q} \int \mathrm{d} \boldsymbol{x}_{0} \prod_{a=1}^{n} \mathrm{~d} \boldsymbol{x}_{a} \times \\
& \times\left\{\phi_{0}\left(\boldsymbol{x}_{0}\right) \prod_{a=1}^{n} \phi\left(\boldsymbol{x}_{a}\right) \prod_{a \geq b} \delta\left(\boldsymbol{x}_{a} \cdot \boldsymbol{x}_{b}-N q_{a b}\right) \|\left(\prod_{a=1}^{n} \prod_{\mu=1}^{M} P\left(y^{\mu} \mid \mathbf{A}^{\mu} \cdot \boldsymbol{x}_{a}\right) \|_{\underline{A}, \boldsymbol{y}}\right\}\right.
\end{aligned}
$$

The expression above can be rewritten in a simplified manner. With the defini- 
tion of the measure

$$
P\left(\boldsymbol{x}_{0},\left\{\boldsymbol{x}_{a}\right\} \mid \mathbf{Q}\right):=\frac{1}{V(\mathbf{Q})} \phi_{0}\left(\boldsymbol{x}_{0}\right) \prod_{a=1}^{n} \phi\left(\boldsymbol{x}_{a}\right) \prod_{a \geq b} \delta\left(\boldsymbol{x}_{a} \cdot \boldsymbol{x}_{b}-N q_{a b}\right),
$$

where $\left\{\boldsymbol{x}_{a}\right\} \equiv\left\{\boldsymbol{x}_{1}, \ldots, \boldsymbol{x}_{n}\right\}$ is the set of replicated variables and $V(\mathbf{Q})$ is the normalization factor

$$
V(\mathbf{Q}) \equiv \int d \boldsymbol{x}_{0} \prod_{a=1}^{n} \mathrm{~d} \boldsymbol{x}_{a} \phi_{0}\left(\boldsymbol{x}_{0}\right) \prod_{a=1}^{n} \phi\left(\boldsymbol{x}_{a}\right) \prod_{a \geq b} \delta\left(\boldsymbol{x}_{a} \cdot \boldsymbol{x}_{b}-N q_{a b}\right)
$$

that guarantees $\int d \boldsymbol{x}_{0} \prod_{a=1}^{n} \mathrm{~d} \boldsymbol{x}_{a} P\left(\boldsymbol{x}_{0},\left\{\boldsymbol{x}_{a}\right\} \mid \mathbf{Q}\right)=1$, expression (2.26) becomes

$$
Z^{(n)}=\int \mathrm{d} \mathbf{Q} V(\mathbf{Q}) \int \mathrm{d} \boldsymbol{x}_{0} \prod_{a=1}^{n} \mathrm{~d} \boldsymbol{x}_{a} P\left(\boldsymbol{x}_{0},\left\{\boldsymbol{x}_{a}\right\} \mid \mathbf{Q}\right)\left\langle\left\langle\prod_{a=1}^{n} \prod_{\mu=1}^{M} P\left(y^{\mu} \mid \mathbf{A}^{\mu} \cdot \boldsymbol{x}_{a}\right)\right\rangle \|_{\underline{A}, \boldsymbol{y}} .\right.
$$

We also define

$$
\xi(\mathbf{Q}):=\int \mathrm{d} \boldsymbol{x}_{0} \prod_{a=1}^{n} \mathrm{~d} \boldsymbol{x}_{a} P\left(\boldsymbol{x}_{0},\left\{\boldsymbol{x}_{a}\right\} \mid \mathbf{Q}\right) \|\left(\prod_{a=1}^{n} \prod_{\mu=1}^{M} P\left(y^{\mu} \mid\left(\mathbf{A} \boldsymbol{x}_{a}\right)_{\mu}\right) \|_{\underline{A}, \boldsymbol{y}},\right.
$$

leading to

$$
Z^{(n)}=\int \mathrm{d} \mathbf{Q}(V(\mathbf{Q}) \times \xi(\mathbf{Q})) .
$$

This means we managed to split the calculation of $\left\langle\left\langle Z^{n}\right\rangle\right\rangle$ into the somewhat simpler integrals $V$ and $\xi$. Since in both cases all variables $\boldsymbol{x}_{a}, a=0,1, \ldots, n$ are integrated, $V$ and $\xi$ are indeed only functions of $\mathbf{Q}$. It was mentioned above that the replicated system partition function could be written $\left\langle\left\langle Z^{n}\right\rangle \sim \int \mu(d \mathbf{Q}) \exp (N g(\mathbf{Q}))\right.$ - the next steps will be directed to calculate $V$ and $\xi$ separately in order to transform (2.31) into an expression in this format.

Let us begin with the calculation of $V(\mathbf{Q})$ (Equation (2.28)). All delta functions in the product $\prod_{a \geq b} \delta\left(\boldsymbol{x}_{a} \cdot \boldsymbol{x}_{b}-N q_{a b}\right)$ can be expanded onto their integral representations

$$
\delta\left(\boldsymbol{x}_{a} \cdot \boldsymbol{x}_{b}-N q_{a b}\right)=\frac{1}{2 \pi} \int_{-i \infty}^{+i \infty} \mathrm{d} \hat{q}_{a b} \exp \left[\hat{q}_{a b}\left(\boldsymbol{x}_{a} \cdot \boldsymbol{x}_{b}-N q_{a b}\right)\right]
$$

and

$$
\delta\left(\left|\boldsymbol{x}_{a}\right|^{2}-N q_{a a}\right)=\frac{1}{4 \pi} \int_{-i \infty}^{+i \infty} \mathrm{d} \hat{q}_{a a} \exp \left[-\frac{1}{2} \hat{q}_{a a}\left(\left|\boldsymbol{x}_{a}\right|^{2}-N q_{a a}\right)\right]
$$


Their substitution in (2.28) results in

$$
\begin{aligned}
V(\mathbf{Q})= & \int \mathrm{d} \boldsymbol{x}_{0} \prod_{a=1}^{n} \mathrm{~d} \boldsymbol{x}_{a} \phi_{0}\left(\boldsymbol{x}_{0}\right) \prod_{a=1}^{n} \phi\left(\boldsymbol{x}_{a}\right) \prod_{a} \delta\left(\boldsymbol{x}_{a} \cdot \boldsymbol{x}_{a}-N q_{a a}\right) \prod_{a>b} \delta\left(\boldsymbol{x}_{a} \cdot \boldsymbol{x}_{b}-N q_{a b}\right) \\
=\int \mathrm{d} \boldsymbol{x}_{0} \prod_{a=1}^{n} \mathrm{~d} \boldsymbol{x}_{a} \int & \prod_{a=0}^{n} \frac{\mathrm{d} \hat{q}_{a a}}{4 \pi} \int \prod_{a>b} \frac{\mathrm{d} \hat{q}_{a b}}{2 \pi} \phi_{0}\left(\boldsymbol{x}_{0}\right) \prod_{a=1}^{n} \phi\left(\boldsymbol{x}_{a}\right) \times \\
& \times \exp \left[\sum_{a>b} \hat{q}_{a b}\left(\boldsymbol{x}_{a} \cdot \boldsymbol{x}_{b}-N q_{a b}\right)-\frac{1}{2} \sum_{a=0}^{n} \hat{q}_{a a}\left(\left|\boldsymbol{x}_{a}\right|^{2}-N q_{a a}\right)\right] .
\end{aligned}
$$

All exponential terms that are explicitly proportional to $N$ can be readily separated:

$$
\begin{aligned}
V(\mathbf{Q})=\int & \prod_{a=0}^{n} \frac{\mathrm{d} \hat{q}_{a a}}{4 \pi} \int \prod_{a>b} \frac{\mathrm{d} \hat{q}_{a b}}{2 \pi} \exp \left(\frac{N}{2} \sum_{a=0}^{n} q_{a a} \hat{q}_{a a}-N \sum_{a>b} \hat{q}_{a b} q_{a b}\right) \times \\
& \times \int \mathrm{d} \boldsymbol{x}_{0} \prod_{a=1}^{n} \mathrm{~d} \boldsymbol{x}_{a} \phi\left(\boldsymbol{x}_{0}\right) \prod_{a=1}^{n} \phi\left(\boldsymbol{x}_{a}\right) \exp \left(-\frac{1}{2} \sum_{a=0}^{n} \hat{q}_{a a}\left|\boldsymbol{x}_{a}\right|^{2}+\sum_{a>b} \hat{q}_{a b} \boldsymbol{x}_{a} \cdot \boldsymbol{x}_{b}\right) .
\end{aligned}
$$

Now, there are no reasons to believe that the statistics of any signal component $x_{0, i}$ depend on the index $i$ - when the signal is generated, its generating distribution makes no distinction between components. The same reasoning can be applied to the replica components $x_{a, i}$. This implies that the last line of expression above, comprising all terms which do not explicitly depend on $N$, can be factorized as

$$
\begin{aligned}
\int \mathrm{d} & \boldsymbol{x}_{0} \prod_{a=1}^{n} \mathrm{~d} \boldsymbol{x}_{a} \phi\left(\boldsymbol{x}_{0}\right) \prod_{a=1}^{n} \phi\left(\boldsymbol{x}_{a}\right) \exp \left(-\frac{1}{2} \sum_{a=0}^{n} \hat{q}_{a a}\left|\boldsymbol{x}_{a}\right|^{2}+\sum_{a>b} \hat{q}_{a b} \boldsymbol{x}_{a} \cdot \boldsymbol{x}_{b}\right) \\
= & \prod_{i=1}^{N} \int \mathrm{d} x_{0, i} \prod_{a=1}^{n} \mathrm{~d} x_{a, i} \phi\left(x_{0, i}\right) \prod_{a=1}^{n} \phi\left(x_{a, i}\right) \exp \left(-\frac{1}{2} \sum_{a=0}^{n} \hat{q}_{a a}\left|x_{a, i}\right|^{2}+\sum_{a>b} \hat{q}_{a b} x_{a, i} \cdot x_{b, i}\right) \\
= & {[\underbrace{\int \mathrm{d} x_{0} \prod_{a=1}^{n} \mathrm{~d} x_{a} \phi\left(x_{0}\right) \prod_{a=1}^{n} \phi\left(x_{a}\right) \exp \left(-\frac{1}{2} \sum_{a=0}^{n} \hat{q}_{a a}\left|x_{a}\right|^{2}+\sum_{a>b} \hat{q}_{a b} x_{a} \cdot x_{b}\right)}_{:=I(\hat{\mathbf{Q}})}]^{N}, }
\end{aligned}
$$

where $\hat{\mathbf{Q}}=\left(\hat{q}_{a b}\right)$. This way,

$$
V(\mathbf{Q})=\int \prod_{a=0}^{n} \frac{\mathrm{d} \hat{q}_{a a}}{4 \pi} \int \prod_{a>b} \frac{\mathrm{d} \hat{q}_{a b}}{2 \pi} \exp N\left(\frac{1}{2} \sum_{a} q_{a a} \hat{q}_{a a}-\sum_{a>b} \hat{q}_{a b} q_{a b}+\log I(\hat{\mathbf{Q}})\right)
$$


Now the first limit can be taken — by taking $N \rightarrow \infty$, Laplace's method ${ }^{5}$ gives

$$
V(\mathbf{Q}) \sim \exp \left\{N \underset{\left\{\hat{q}_{a a}\right\},\left\{\hat{q}_{a b}\right\}}{\operatorname{extr}}\left[\frac{1}{2} \sum_{a=0}^{n} q_{a a} \hat{q}_{a a}-\sum_{a>b} \hat{q}_{a b} q_{a b}+\log I(\hat{\mathbf{Q}})\right]\right\},
$$

so that the only relevant contribution to the integral (2.35) comes from the factor which includes the variables $\left\{\hat{q}_{a a}\right\}$ and $\left\{\hat{q}_{a b}\right\}$ which maximize $\sum_{a} q_{a a} \hat{q}_{a a} / 2-$ $\sum_{a>b} \hat{q}_{a b} q_{a b}+\log I(\hat{\mathbf{Q}})$. Finding these terms can be difficult, so we must enforce some symmetry over them, reducing the number of variables and effectively simplifying all related expressions.

\section{Replica Symmetric Ansatz}

To consider replica symmetric solutions means identifying $\mathbf{Q}$ and $\hat{\mathbf{Q}}$ according to:

$$
q_{a b}=q_{b a}= \begin{cases}Q_{0}, & \text { for } a=b=0, \\ m, & \text { for } a=0 \text { and } b \neq 0 ; a \neq 0 \text { and } b=0, \\ Q, & \text { for } a=b=1,2, \ldots, n, \\ q, & \text { for } a \neq b \text { and } a, b \neq 0,\end{cases}
$$

and

$$
\hat{q}_{a b}=\hat{q}_{b a}= \begin{cases}0, & \text { for } a=b=0, \\ \hat{m}, & \text { for } a=0 \text { and } b \neq 0 ; a \neq 0 \text { and } b=0, \\ \hat{Q}, & \text { for } a=b=1,2, \ldots, n, \\ \hat{q}, & \text { for } a \neq b \text { and } a, b \neq 0 .\end{cases}
$$

With these, the exponents in (2.37) can be simplified to

$$
\begin{aligned}
\frac{1}{2} \sum_{a} \hat{q}_{a a} q_{a a}-\sum_{a>b} \hat{q}_{a b} q_{a b} \\
\quad=\frac{1}{2} \hat{q}_{00} q_{00}+\frac{1}{2} \sum_{a=1}^{n} \hat{q}_{a a} q_{a a}-\sum_{a=1}^{n} \hat{q}_{a 0} q_{a 0}-\sum_{a=b}^{n} \sum_{b=1}^{n} \hat{q}_{a b} q_{a b} \\
\quad=0+\frac{1}{2} n \hat{Q} Q-n \hat{m} m-\frac{1}{2} n(n-1) \hat{q} q,
\end{aligned}
$$

${ }^{5}$ A method for approximations of integrals due to Laplace [77]:

$$
\lim _{N \rightarrow \infty} \int d x e^{N f(x)}=e^{N \operatorname{extr}_{x} f(x)}
$$


as well as the term inside $I(\hat{\mathbf{Q}})$, which becomes

$$
\begin{aligned}
& -\frac{1}{2} \sum_{a=0}^{n} \hat{q}_{a a}\left(x_{a}\right)^{2}+\sum_{a>b} \hat{q}_{a b} x_{a} x_{b} \\
& \quad=-\frac{1}{2} \hat{q}_{00}\left(x_{0}\right)^{2}-\frac{1}{2} \sum_{a=1}^{n} \hat{q}_{a a}\left(x_{a}\right)^{2}+\sum_{a=1}^{n} \hat{q}_{a 0} x_{a} x_{0}+\sum_{a=b}^{n} \sum_{b=1}^{n} \hat{q}_{a b} x_{a} x_{b} \\
& \quad=0-\frac{1}{2} \hat{Q} \sum_{a=1}^{n}\left(x_{a}\right)^{2}+\hat{m} x_{0} \sum_{a=1}^{n} x_{a}+\hat{q} \sum_{a=b}^{n} \sum_{b=1}^{n} x_{a} x_{b} .
\end{aligned}
$$

By using the Hubbard-Stratonovich transformation,

$$
\exp \left(\hat{q} \sum_{a>b} x_{a} x_{b}\right)=\int \mathcal{D} t \exp \left[\sum_{a}\left(-\frac{\hat{q}}{2}\left(x_{a}\right)^{2}+\sqrt{\hat{q}} t x_{a}\right)\right]
$$

with $\mathcal{D} t=(1 / \sqrt{2 \pi}) \exp \left(-t^{2} / 2\right) d t$, we can write $I(\hat{\mathbf{Q}})$ as

$$
\begin{aligned}
I(\hat{\mathbf{Q}})= & \log \int \mathrm{d} x_{0} \prod_{a=1}^{n} \mathrm{~d} x_{a} \phi\left(x_{0}\right) \prod_{a=1}^{n} \phi\left(x_{a}\right) \exp \left(-\frac{1}{2} \sum_{a=0}^{n} \hat{q}_{a a} x_{a}^{2}+\sum_{a>b} \hat{q}_{a b} x_{a} x_{b}\right) \\
= & \log \int \mathrm{d} x_{0} \prod_{a=1}^{n} \mathrm{~d} x_{a} \phi\left(x_{0}\right) \prod_{a=1}^{n} \phi\left(x_{a}\right) \\
& \times \int \mathcal{D} t \exp \left[\sum_{a}\left(-\frac{\hat{Q}}{2}\left(x_{a}\right)^{2}-\frac{\hat{q}}{2}\left(x_{a}\right)^{2}+\sqrt{\hat{q}} t x_{a}+\hat{m} x_{0} x_{a}\right)\right] \\
= & \log \int \mathrm{d} x_{0} \phi\left(x_{0}\right) \int \mathcal{D} t \prod_{a=1}^{n}\left[\int \mathrm{d} x_{a} \phi\left(x_{a}\right) \exp \left(-\frac{\hat{Q}+\hat{q}}{2} x_{a}^{2}+\left(\sqrt{\hat{q}} t+m x_{0}\right) x_{a}\right)\right] .
\end{aligned}
$$

Now, each and every one of these integrals in the product on the R.H.S. is identical. This means we can substitute the product for the $n$-th power of one of these integrals:

$$
\begin{aligned}
& \prod_{a=1}^{n} \int \mathrm{d} x_{a} \phi\left(x_{a}\right) \exp \left(-\frac{\hat{Q}+\hat{q}}{2} x_{a}^{2}+\left(\sqrt{\hat{q}} t+m x_{0}\right) x_{a}\right)= \\
& {\left[\int \mathrm{d} x \phi(x) \exp \left(-\frac{\hat{Q}+\hat{q}}{2} x^{2}+\left(\sqrt{\hat{q}} t+m x_{0}\right) x\right)\right]^{n} }
\end{aligned}
$$

and advance the fact that $n$ will be very small at the end of the calculation to already simplify even more the expression for $V(\mathbf{Q})$. Defining

$$
\Omega\left(\hat{Q}, \hat{q}, \hat{m} ; x_{0}\right) \equiv \int \mathrm{d} x \phi(x) \exp \left[-\frac{\hat{Q}+\hat{q}}{2} x^{2}+\left(\sqrt{\hat{q}} t+\hat{m} x_{0}\right) x\right]
$$


it is possible to consider that $n \ll 1$ over $I(\hat{\mathbf{Q}})$ :

$$
\begin{aligned}
I(\hat{\mathbf{Q}}) & =\log \int \mathrm{d} x_{0} \phi\left(x_{0}\right) \int \mathcal{D} t \exp \left[n \log \Omega\left(\hat{Q}, \hat{q}, \hat{m} ; x_{0}\right)\right] \\
& \approx \log \int \mathrm{d} x_{0} \phi\left(x_{0}\right) \int \mathcal{D} t\left[1+n \log \Omega\left(\hat{Q}, \hat{q}, \hat{m} ; x_{0}\right)\right] \\
& =\log \left[1+n \int \mathrm{d} x_{0} \phi\left(x_{0}\right) \int \mathcal{D} t \log \Omega\left(\hat{Q}, \hat{q}, \hat{m} ; x_{0}\right)\right] \\
& \approx n \int \mathrm{d} x_{0} \phi\left(x_{0}\right) \int \mathcal{D} t \log \Omega\left(\hat{Q}, \hat{q}, \hat{m} ; x_{0}\right)
\end{aligned}
$$

The final expression for $V(\mathbf{Q})$ is then

$$
\begin{aligned}
V(\mathbf{Q})= & \exp N \operatorname{extr} \\
& \left\{\frac{n}{2} \hat{Q}, \hat{q}, \hat{m}\right. \\
& \left.\operatorname{er}-\frac{n(n-1)}{2} \hat{q} q-n \hat{m} m+n \int \mathrm{d} x_{0} \phi\left(x_{0}\right) \int \mathcal{D} t \Omega\left(\hat{Q}, \hat{q}, \hat{m} ; x_{0}\right)\right\} .
\end{aligned}
$$

Now we turn to the calculation of the second integral, $\xi(\mathbf{Q})$, defined in (2.30) and restated here as

$$
\xi(\mathbf{Q})=\left\langle\left\langle\prod_{a=1}^{n} \prod_{\mu=1}^{M} P\left(y^{\mu} \mid \mathbf{A}^{\mu} \cdot \boldsymbol{x}_{a}\right) \|_{\underline{A}, \boldsymbol{y},\left\{\boldsymbol{x}_{a}\right\}, \boldsymbol{x}_{0}},\right.\right.
$$

where the averages over all $\left\{\boldsymbol{x}_{a}\right\}$ and $\boldsymbol{x}_{0}$ are taken with respect to the metric $P\left(\boldsymbol{x}_{0},\left\{\boldsymbol{x}_{a}\right\} \mid \mathbf{Q}\right)$. Using arguments similar to above and attributing invariance with respect to measurements $\mu$, this becomes

$$
\xi(\mathbf{Q})=\left[\left\langle\left\langle\prod_{a=1}^{n} P\left(y \mid \mathbf{A} \cdot \boldsymbol{x}_{a}\right)\right\rangle \|_{\underline{A}, \boldsymbol{y},\left\{\boldsymbol{x}_{a}\right\}, \boldsymbol{x}_{0}}\right]^{M} .\right.
$$

But all dependence on $\underline{A}$ and $\boldsymbol{x}_{a}$ is actually stated through their dot product,

$$
u_{a}^{\mu}=\mathbf{A}^{\mu} \cdot \boldsymbol{x}_{a}=\sum_{i=1}^{N} A_{i}^{\mu} x_{a, i}
$$

Because all $A_{i}^{\mu}$ are i.i.d. variables distributed as $\mathcal{N}(0,1 / N)$, the central limit theorem tells us that the aggregated variable $u_{a}^{\mu}$ is also a Gaussian variable with mean 0 and 
covariance

$$
\left\langle u_{a}^{\mu} u_{b}^{\nu}\right\rangle_{\mathbf{A}}=\sum_{i j} \underbrace{\left\langle A_{i}^{\mu} A_{j}^{\nu}\right\rangle_{\mathbf{A}}}_{\frac{1}{N} \delta_{\mu \nu} \delta_{i j}} x_{a, i} x_{b, j}=\delta_{\mu \nu} \frac{\sum_{i} x_{a, i} x_{b, i}}{N}=\delta_{\mu \nu} q_{a b},
$$

so that $\xi(\mathbf{Q})$ can finally be written as an average uniquely over the variables $u_{a}$ :

$$
\xi(\mathbf{Q})=\left[\left\langle\iint d y P\left(y \mid u_{0}\right) \prod_{a=1}^{n} P\left(y \mid u_{a}\right)\|\|_{\left\{u_{a}\right\}}\right]^{M} .\right.
$$

Now note that property (2.58) dictates that the variables $u_{0}, \ldots, u_{n}$ can be written as the sum of the standard Gaussian random variables $w$ and $s_{0}, \ldots, s_{n}$ in the following way:

$$
\begin{aligned}
& u_{0}=\sqrt{Q_{0}-\frac{m^{2}}{q}} s_{0}+\frac{m}{\sqrt{q}} w \\
& u_{a}=\sqrt{Q-q} s_{a}+\sqrt{q} w, \quad(a=1,2 \ldots, n),
\end{aligned}
$$

where the Replica Symmetric ansatz was taken into account. With these we have an explicit expression for the averages in (2.59):

$$
\begin{aligned}
\xi(\mathbf{Q})= & {\left[\int d y \int \mathcal{D} w \int \mathcal{D} s_{0} \prod_{a=1}^{n} \mathcal{D} s_{a} \times\right.} \\
& \left.\times P\left(y \mid \sqrt{Q_{0}-\frac{m^{2}}{q}} s_{0}+\frac{m}{\sqrt{q}} w\right) \prod_{a=1}^{n} P\left(y^{\mu} \mid \sqrt{Q-q} s_{a}+\sqrt{q} w\right)\right]^{M} \\
= & {\left[\int d y \int \mathcal{D} w \int \mathcal{D} s_{0} \times\right.} \\
& \left.\times P\left(y \mid \sqrt{Q_{0}-\frac{m^{2}}{q}} s_{0}+\frac{m}{\sqrt{q}} w\right)\left(\int \mathcal{D} s P\left(y^{\mu} \mid \sqrt{Q-q} s+\sqrt{q} w\right)\right)^{n}\right]^{M}
\end{aligned}
$$

where, once again, replica invariance was taken into account.

\section{FREE ENERGY}

Now that expressions for $V(\mathbf{Q})=V\left(Q, q, m, Q_{0}\right)$ and $\xi(\mathbf{Q})=\xi\left(Q, q, m, Q_{0}\right)$ are available, we can go further and calculate the actual free energy density (2.16) by making use of the replica trick (2.18), which can be restated as

$$
\bar{f} \equiv \lim _{N \rightarrow \infty} N^{-1} \lim _{n \rightarrow 0} \frac{\partial}{\partial n} \log \left\langle\left\langle Z^{n}\right\rangle\right\rangle
$$


for $\beta=1$. With the factorization $(2.31), \log \left\langle\left\langle Z^{n}\right\rangle / N\right.$ can be written

$$
\begin{aligned}
\frac{1}{N} \log \left\langle\left\langle Z^{n}\right\rangle\right. & =\frac{1}{N} \log \int \mathrm{d} \mathbf{Q}(V(\mathbf{Q}) \times \xi(\mathbf{Q})) \\
& =\frac{1}{N} \log \int \mathrm{d} \mathbf{Q} \exp \log (V(\mathbf{Q}) \xi(\mathbf{Q})) \\
& =\frac{1}{N} \log \int \mathrm{d} \mathbf{Q} \exp N\left(\frac{1}{N} \log V(\mathbf{Q})+\frac{1}{N} \log \Xi(\mathbf{Q})\right) .
\end{aligned}
$$

Once again we make use of Laplace's method to get an asymptotic approximation for this expression in the limit $N \rightarrow \infty$, so that

$$
\frac{1}{N} \log \left\langle\left\langle Z^{n}\right\rangle\right\rangle \frac{1}{N} \log \exp N \underset{\mathbf{Q}=\{Q, m, q\}}{\operatorname{extr}}\left(\frac{1}{N} \log V(\mathbf{Q})+\frac{1}{N} \log \Xi(\mathbf{Q})\right)
$$

and

$$
\bar{f}=\underset{Q, m, q}{\operatorname{extr}} \lim _{n \rightarrow 0} \frac{\partial}{\partial n}\left(\frac{1}{N} \log V(\mathbf{Q})+\frac{1}{N} \log \Xi(\mathbf{Q})\right),
$$

after inverting the order of the limits on $n$ and $N$. Now we can also get explicit expressions for the R.H.S. terms above using the results (2.54) and (2.61). Starting with $(1 / N) \log V(\mathbf{Q})$, its calculation is straightforward:

$$
\begin{aligned}
& \frac{1}{N} \log V(\mathbf{Q})= \\
& \underset{\hat{Q}, \hat{q}, \hat{m}}{\operatorname{extr}}\left[\frac{n}{2} \hat{Q} Q-\frac{n(n-1)}{2} \hat{q} q-n \hat{m} m+n \int \mathrm{d} x_{0} \phi\left(x_{0}\right) \int \mathcal{D} t \Omega\left(\hat{Q}, \hat{q}, \hat{m} ; x_{0}\right)\right]
\end{aligned}
$$

and

$$
\lim _{n \rightarrow 0} \frac{\partial}{\partial n} \frac{1}{N} \log V(\mathbf{Q})=\underset{\hat{Q}, \hat{q}, \hat{m}}{\operatorname{extr}}\left\{\frac{1}{2} \hat{Q} Q+\frac{1}{2} \hat{q} q-\hat{m} m+\int \mathrm{d} x_{0} \phi\left(x_{0}\right) \int \mathcal{D} t \Omega\left(\hat{Q}, \hat{q}, \hat{m} ; x_{0}\right)\right\} .
$$


The second term, $(1 / N) \log \xi(\mathbf{Q})$, is less direct and proceeds as follows:

$$
\begin{aligned}
\frac{1}{N} \log \xi(\mathbf{Q})=\frac{M}{N} \log \{ & \int d y \int \mathcal{D} w \int \mathcal{D} s_{0} \times \\
& \left.\times P\left(y \mid \sqrt{Q_{0}-\frac{m^{2}}{q}} s_{0}+\frac{m}{\sqrt{q}} w\right)(\mathcal{D} s P(y \mid \sqrt{Q-q} s+\sqrt{q} w))^{n}\right\} \\
= & \alpha \log \left\{\int d y \int \mathcal{D} w \int \mathcal{D} s_{0} \times\right. \\
\simeq & \alpha \log \left\{\int d y \int \mathcal{D} w \int \mathcal{D} s_{0} \times\right. \\
\times P(y \mid & \left.\left.\left.\left.\sqrt{Q_{0}-\frac{m^{2}}{q}} s_{0}+\frac{m}{\sqrt{q}} w\right) e^{n \log (\mathcal{D} s P(y \mid \sqrt{Q-q} s+\sqrt{q} w))}\right\}\right)[1+n \log (\mathcal{D} s P(y \mid \sqrt{Q-q} s+\sqrt{q} w))]\right\} \\
= & \alpha \log \left[1+n \int d y \int \mathcal{D} w \int \mathcal{D} s_{0} \times\right. \\
& \left.\times P\left(y \mid \sqrt{Q_{0}-\frac{m^{2}}{q}} s_{0}+\frac{m}{\sqrt{q}} w\right) \log (\mathcal{D} s P(y \mid \sqrt{Q-q} s+\sqrt{q} w))\right] \\
\simeq & \alpha n \int d y \int \mathcal{D} w \int \mathcal{D} s_{0} \times \\
& \times P\left(y \mid \sqrt{Q_{0}-\frac{m^{2}}{q}} s_{0}+\frac{m}{\sqrt{q}} w\right) \log (\mathcal{D} s P(y \mid \sqrt{Q-q} s+\sqrt{q} w)),
\end{aligned}
$$

so that

$$
\begin{aligned}
\lim _{n \rightarrow 0} \frac{\partial}{\partial n} \frac{1}{N} \log \xi(\mathbf{Q}) \approx \alpha \int d y \int \mathcal{D} w \int \mathcal{D} & s_{0} P\left(y \mid \sqrt{Q_{0}-\frac{m^{2}}{q}} s_{0}+\frac{m}{\sqrt{q}} w\right) \times \\
& \times \log (\mathcal{D} s P(y \mid \sqrt{Q-q} s+\sqrt{q} w))
\end{aligned}
$$

Therefore, joining (2.67) and (2.68) and defining $\Theta \equiv\{Q, m, q, \hat{Q}, \hat{q}, \hat{m}\}$, the free energy density is, up to an additive factor, equal to

$$
\begin{array}{r}
\bar{f}=-\operatorname{extr}_{\Theta}\left\{\frac{1}{2} \hat{Q} Q+\frac{1}{2} \hat{q} q-\hat{m} m+\int \mathrm{d} x_{0} \phi\left(x_{0}\right) \int \mathcal{D} t \Omega\left(\hat{Q}, \hat{q}, \hat{m} ; x_{0}\right)+\right. \\
+\alpha \int d y \int \mathcal{D} w \int \mathcal{D} s_{0}\left[P\left(y \mid \sqrt{Q_{0}-\frac{m^{2}}{q}} s_{0}+\frac{m}{\sqrt{q}} w\right) \times\right. \\
\left.\left.\times \log \left(\int \mathcal{D} s P(y \mid \sqrt{Q-q} s+\sqrt{q} w)\right)\right]\right\} .
\end{array}
$$


This result is general - given any measurement model $P(y \mid u)$, any signal generating distribution $\phi_{0}(x)$, and any prior $\phi(x)$, the R.H.S. can be extremized over the small number of replica overlap parameters. The result would then correspond to the free energy of this infinite thermodynamical system.

But what do all this say about the inference procedure? To answer this, note that the expected mse normalized by $\left\|\boldsymbol{x}^{0}\right\|^{2}$ can be written

$$
\|\left(\frac{\left\|x_{0}-x\right\|^{2}}{\left\|x_{0}\right\|^{2}} \|=\left\langle\left\langle\frac{x_{0} \cdot x_{0}-2 x_{0} x+x \cdot x}{x_{0} \cdot x_{0}} \|=\frac{Q_{0}-2 m+q}{Q_{0}} .\right.\right.\right.
$$

For any given $\alpha$ and $\rho$, the parameters which extremize the free energy (2.69) will assume particular values, which are directly connected to the reconstruction error given by (2.70). The expressions that describe the replica overlap variables in the extremum of (2.69) are called saddle-point equations. Now consider the scenario where the signal generating distribution is known, i.e. $\phi(x)=\phi_{0}(x) .{ }^{6}$ Knowledge of $\phi_{0}(x)$ allows us to enforce that any solution $\boldsymbol{x}$ to the inference problem has the same expected moments as the signal. According to the definition (2.24) for the replica overlaps $q_{a b}$ and the replica symmetrical conditions (2.38) and (2.39), this means

$$
Q=Q_{0} \quad \text { and } \quad q=m
$$

and

$$
\hat{Q}=\hat{q}=\hat{m}
$$

Let us define

$$
\begin{aligned}
\Gamma(y, q):=\int \mathcal{D} w \int \mathcal{D} s_{0}[ & P\left(y \mid \sqrt{Q_{0}-\frac{m^{2}}{q}} s_{0}+\frac{m}{\sqrt{q}} w\right) \times \\
& \left.\times \log \left(\int \mathcal{D} s P(y \mid \sqrt{Q-q} s+\sqrt{q} w)\right)\right] .
\end{aligned}
$$

in such a way that considering the conditions (2.71) and (2.72), the free energy becomes the simpler expression

$$
\bar{f}=-\operatorname{extr}_{q, \hat{q}}\left[\frac{1}{2} \hat{q} q+\int \mathrm{d} x_{0} \phi\left(x_{0}\right) \int \mathcal{D} t \Omega\left(t, \hat{q} ; x_{0}\right)+\alpha \int d y \Gamma(y, q)\right] \text {. }
$$

In the Nishimori line then, the normalized mse becomes mse $=1-q / Q_{0}$, where $q$

\footnotetext{
${ }^{6}$ In this case, we say that we are on the Nishimori line [67].
} 
comes from the extremization of (2.74), i.e. from solving the two-variable system:

$$
\left\{\begin{array}{l}
\hat{q}=-2 \alpha(\partial / \partial q) \int d y \Gamma(y, q) \\
q=2(\partial / \partial \hat{q}) \int d x_{0} \phi\left(x_{0}\right) \int \mathcal{D} t \Omega\left(t, \hat{q} ; x_{0}\right)
\end{array}\right.
$$

which summarizes the Bayesian Compressed Sensing inference process.

\section{SAdDle-Point Equations}

In what follows, we will show the explicit form of the saddle-point equations for the noiseless standard Compressed Sensing scenario described by the distribution $P(y \mid u)=\delta(y-u)$, as defined previously. As usual, we consider signals generated by the Gauss-Bernoulli distribution $\phi_{0}(x)=(1-\rho) \delta(x)+\rho \exp \left(-x^{2} / 2\right) \sqrt{2 \pi}$.

Take for example the R.H.S. of the first equation of (2.75). With $\int \mathcal{D} s P(y \mid X s+$ $Y)=(\sqrt{2 \pi} X)^{-1} \exp \left[-\frac{1}{2}\left(\frac{y-Y}{X}\right)^{2}\right]$, the integral $\Gamma(y, q)$ becomes

$$
\begin{aligned}
& \Gamma(y, q)= \int \mathcal{D} w\left\{\frac{1}{\sqrt{2 \pi(\rho-q)}} \exp \left[-\frac{1}{2(\rho-q)}(y-\sqrt{q} w)^{2}\right] \times\right. \\
&\left.\quad \times\left[-\frac{1}{2} \log \left[2 \pi\left(Q_{0}-q\right)\right]-\frac{1}{2\left(Q_{0}-q\right)}(y-\sqrt{q} w)^{2}\right]\right\} \\
&=-\frac{1}{2 \sqrt{2 \pi(\rho-q)}}\left[\log \left[2 \pi\left(Q_{0}-q\right)\right] I_{0}+\frac{1}{Q_{0}-q}\left(y^{2} I_{0}+q I_{2}-2 \sqrt{q} y I_{1}\right)\right],
\end{aligned}
$$

where we defined the integrals

$$
\begin{aligned}
I_{p} & \equiv \int \mathcal{D} w w^{p} \exp \left[-\frac{1}{2(\rho-q)}(y-\sqrt{q} w)^{2}\right] \\
& =e^{-\frac{1}{2 \rho} y^{2}}\left(\frac{\rho-q}{\rho}\right)^{p / 2} \frac{1}{\sqrt{2 \pi}} \int d w w^{p} e^{-\frac{1}{2}\left(w-\frac{y \sqrt{q}}{\sqrt{\rho(\rho-q)}}\right)^{2}} .
\end{aligned}
$$

For $p=0,1,2$, they have straightforward closed formulas:

$$
\begin{aligned}
& I_{0}=\sqrt{\frac{\rho-q}{\rho}} \exp \left(-\frac{1}{2 \rho} y^{2}\right) \\
& I_{1}=\left(\sqrt{\frac{\rho-q}{\rho}}\right)^{2} \exp \left(-\frac{1}{2 \rho} y^{2}\right) \frac{y \sqrt{q}}{\sqrt{\rho(\rho-q)}} \\
& I_{2}=\left(\sqrt{\frac{\rho-q}{\rho}}\right)^{3} \exp \left(-\frac{1}{2 \rho} y^{2}\right)\left[1+\frac{y^{2} q}{\rho(\rho-q)}\right],
\end{aligned}
$$


whose introduction in (2.77) leads to

$$
\Gamma(y, q)=-\frac{1}{2} \frac{1}{\sqrt{2 \pi \rho}} e^{-\frac{1}{2 \rho} y^{2}}\left[\frac{1}{Q_{0}-q}\left(\frac{\rho-q}{\rho}\right)^{2} y^{2}+\log \left[2 \pi\left(Q_{0}-q\right)\right]+\frac{q(\rho-q)}{\rho\left(Q_{0}-q\right)}\right]
$$

Now, integration of this quantity is simple:

$$
\begin{aligned}
\int \mathrm{d} y \Gamma(y, q)= & -\frac{1}{2\left(Q_{0}-q\right)}\left(\frac{\rho-q}{\rho}\right)^{2} \overbrace{\frac{1}{\sqrt{2 \pi \rho}} \int \mathrm{d} y y^{2} \exp \left(-\frac{1}{2 \rho} y^{2}\right)}^{=\rho} \\
& -\frac{1}{2}\left\{\frac{q(\rho-q)}{\rho\left(Q_{0}-q\right)}+\log \left[2 \pi\left(Q_{0}-q\right)\right]\right\} \underbrace{\frac{1}{\sqrt{2 \pi \rho}} \int \mathrm{d} y y^{2} \exp \left(-\frac{1}{2 \rho} y^{2}\right)}_{=1} \\
= & \left.-\frac{1}{2}\left[\frac{q(\rho-q)}{\rho\left(Q_{0}-q\right)}+\log 2 \pi+\log \left(Q_{0}-q\right)\right]+\frac{\rho}{Q_{0}-q}\left(\frac{\rho-q}{\rho}\right)^{2}\right] \\
= & \left.-\frac{1}{2}\left[\frac{\rho-q}{Q_{0}-q} \log 2 \pi+\log \left(Q_{0}-q\right)\right]\right]
\end{aligned}
$$

and its posterior derivative straightforward:

$$
\frac{\partial}{\partial q} \int d y \Gamma(y, q)=-\frac{1}{2} \frac{\rho-q-2\left(Q_{0}-q\right)}{\left(Q_{0}-q\right)^{2}}=\frac{1}{2(\rho-q)}
$$

where the last step follows from the identity $Q_{0} \equiv \rho$. The R.H.S. of the second equation of the system (2.75) can be calculated through similar methods, giving rise to the saddle-point equations

$$
\left\{\begin{array}{l}
\hat{q}=\frac{\alpha}{\rho-q} \\
q=\rho-\frac{1}{1+\hat{q}}\left[1+\hat{q}-\rho \hat{q} \int \mathcal{D} z z^{2}\left((1-\rho) \sqrt{1+\hat{q}} e^{-\hat{q} z^{2} / 2}+\rho\right)\right]
\end{array}\right.
$$

The system (2.85) can be solved for all $0 \leq \alpha, \rho \leq 1$. It turns out that for any given $\rho$, there is an $\alpha_{c}$ such that for all $\alpha \geq \alpha_{c}$, the parameter $q$ is equal to $Q_{0}$. The normalized error mse $=1-q / Q_{0}$ then becomes null, implying perfect reconstruction. An example is shown in Figure 2.2, where the values of $q, \hat{q}$ and the normalized mse for $\rho=0.4$ and crescent $\alpha$ are shown. Note there the presence of $\alpha_{c} \simeq 0.59$ (blue vertical dashed line) for which $q \rightarrow Q_{0}$ and $\hat{q} \rightarrow \infty$. Figure 2.3 shows a phase-diagram for the reconstruction error of the Bayesian scheme in the noiseless standard CS setting. The colors, just like the diagram on the previous chapter for the $\ell_{1}$-minimization problem, represent the resulting mean error of 100 simulations of the AMP algorithm for each pair $(\alpha, \rho)$ with signal size $N=500$. The sharp 
transition line (in black) separating the upper left part (where the reconstruction error is zero) from the bottom right part (of non-zero error) corresponds to the $\alpha_{c}(\rho)$ value found by solving the saddle-point equations (2.85). Note the exact match between theoretical prescription and empirical results. ${ }^{7}$

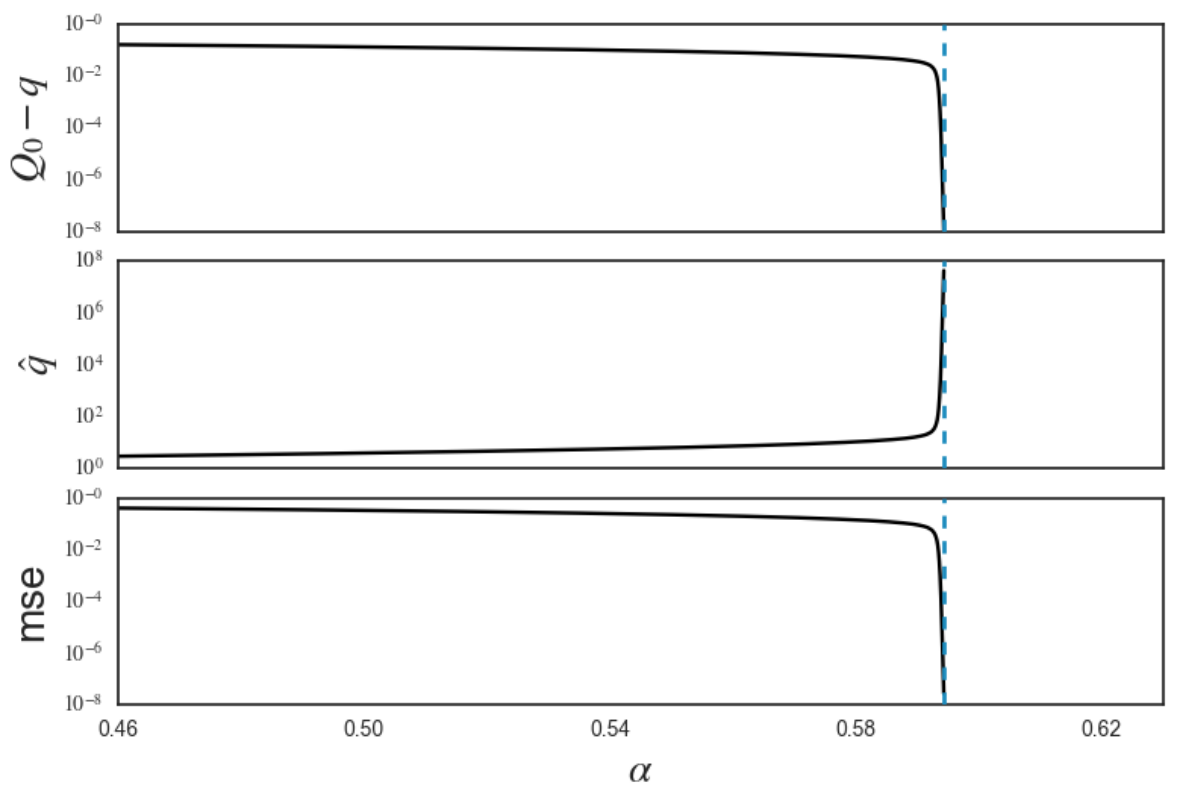

Figure 2.2: Order parameters for $\rho=0.4$ in the noiseless standard Compressed Sensing scenario. The blue dashed vertical lines correspond to $\alpha_{c}(\rho=0.4) \simeq 0.59$, the critical value for which $q \rightarrow Q_{0}, \hat{q} \rightarrow \infty$ and mse $\rightarrow 0$, denoting a phase transition.

These results show that the replica method, even without rigorous mathematical proof, proves itself a valuable analytical tool for the understanding of typicality in inference systems - in particular, Compressed Sensing recovery schemes. While we have not proved the stability of the replica symmetric saddle-point (the interested reader can see the proof on $[74,137])$, the convergence of experimental results and the replica symmetric prescription is very telling. In the next chapter the Bayesian Online Compressed Sensing algorithm will be introduced. The theoretical prescriptions of this chapter will serve as a starting point for performance comparisons between the offline and the online frameworks.

${ }^{7}$ The curves in Figure 2.2 can be seen as a vertical slice of this diagram, where for fixed $\rho$ one would go up through crescent values of $\alpha$ until the value $\alpha_{c}(\rho)$ is reached, for which the error drops to zero, following $q \rightarrow Q_{0}$ and $\hat{q} \rightarrow \infty$. 


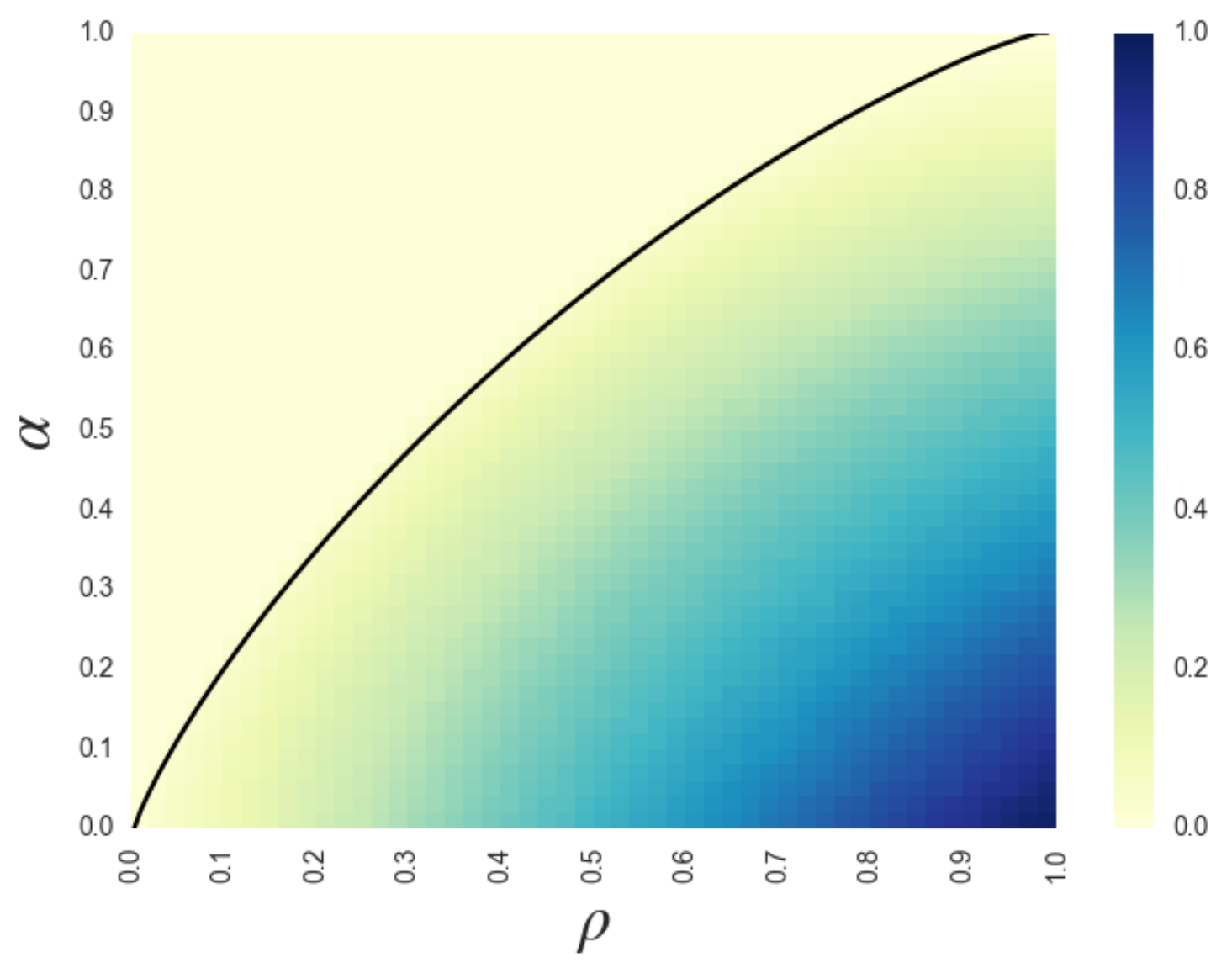

Figure 2.3: Phase diagram of the Bayesian reconstruction (2.3) for the noiseless standard Compressed Sensing problem $P(y \mid u)=\delta(y-u)$ with Gauss-Bernoulli generating distribution $\phi_{0}(x)=(1-\rho) \delta(x)+\rho \exp \left(-x^{2} / 2\right) \sqrt{2 \pi}$, which is known and equal to the prior $\phi(x)$. Each pair $(\alpha, \rho)$ color corresponds to the average normalized reconstruction error $\mathrm{e}=(1 / N)\left\|\hat{\boldsymbol{x}}-\boldsymbol{x}_{0}\right\|_{2}^{2} /\left\|\boldsymbol{x}_{0}\right\|^{2}$ resulting of 100 simulations of the AMP algorithm (Appendix A). with $N=500$. The black curve corresponds to $\alpha_{c}(\rho)$ found through solution of the system of equations (2.85). Note how the theoretical description of the replica method for $N \rightarrow \infty$ matches with great accuracy the results obtained empirically with the AMP algorithm, demarcating two well-separated phases (of zero and non-zero reconstruction error). 



\section{Chapter 3}

\section{Online Compressed Sensing}

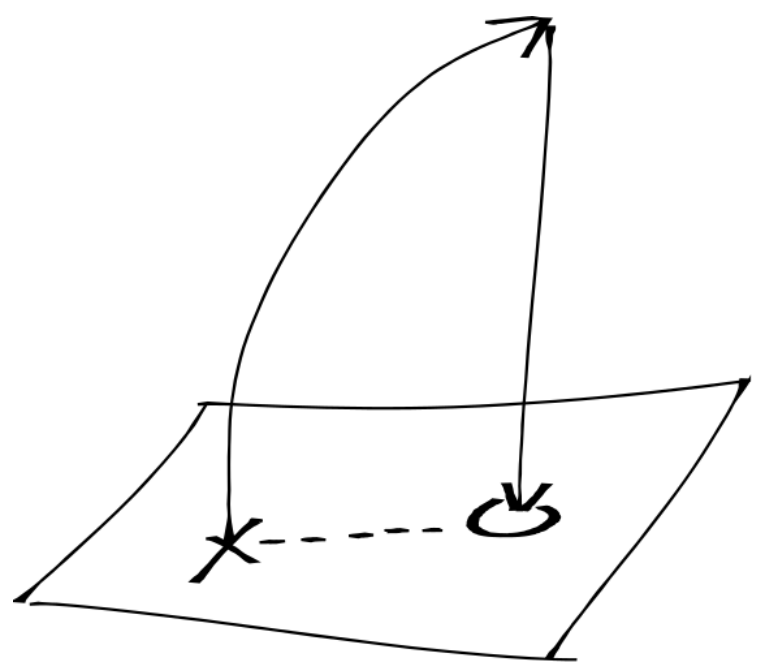

Rossi, Paulo V., Yoshiyuki Kabashima, and Jun-ichi Inoue Bayesian online compressed sensing Physical Review E 94.2 (2016): 022137 


\subsection{INTRODUCTION}

The Compressed Sensing framework is successful in its attempt to reduce the number of measurements needed to capture all the relevant data in a sparse signal. CS techniques compress the sparse signal into a small number of components from which it can then be retrieved by a inference algorithm, be it a general algorithm such as the LASSO or a tailor-made one such as the Bayesian AMP. The catalog of sparse signal reconstruction algorithms grows by the day, and the multitude of options available in the literature covers from general scenarios to very specific cases that might be found in real-world situations.

In this chapter we add another technique to the already available milieu. All algorithms presented until now in this work are what we call batch or off-line algorithms, i.e. all signal measurements that were captured remain available during the entire reconstruction, in a batch from where they can be re-assessed as many times as one would like. But in many practical situations this is not the case. Measurement information come and go for a variety of reasons. For example, storage can be very expensive in low-end devices, so one would like to learn the most they can from a given example and then erase it from memory to give space to new measurements. Or perhaps examples may not be available for long simply because any specific information about them decays with time. This is a common scenario in biological systems, where an entity's cognition might be forever altered by an event whose details have simply vanished from memory - there was learning, but any specific information obtained from the environment at the moment of the "measurement" is lost or mixed in the biological structure of the brain. In other scenarios, one might want to forget old examples. Imagine an environment that changes in time. There is not much to be gained from revisiting old information, from the time the rules were different. In this scenario, ideally one would forget anything that is not relevant for the present situation.

Algorithms where information obtained through a measurement can be used only once (and before the following measurement takes place) are called online algorithms. Any online learning scheme is defined by the elimination of all information regarding any previous measurements, except for what is already encoded in the present state of the inference. Alas, the class of online algorithms made specifically for Compressed Sensing scenarios is severely lacking. In the neural networks and machine learning communities, learning from examples in an online manner is a very common technique which remains relevant [86, 135], but even though the need for online sparse recovery through Compressed Sensing has been hinted at times, to the 
best of our knowledge, a Bayesian method for doing so has not yet been presented.

In this section a general framework for Bayesian online learning will be introduced, followed by the definition of the Bayesian Online Compressed Sensing algorithm [105], which takes the online learning framework into the CS context. In the analysis of the algorithm, it will be shown that the explicit introduction of sparsity information in the prior distribution allows for very accurate signal reconstruction. For ease of reading, some of the concepts already introduced in the sections above will be redefined.

Two things are important to keep in mind here. First, in online learning one has to give up any expectation of perfect signal reconstruction. This is the price to pay for an algorithm capable of running on hardware with significant computational and storage limitations. Appendix B introduces a CS framework specifically made to run on low-memory devices - 1-bit Compressed Sensing. Here we take this situation to a more extreme scenario by taking a further step in this direction: even less memory required with strong limitations on the number of calculations necessary for reconstruction. Second, the collateral effect of lowering the memory needed to accurately represent the signal is the simultaneous increase in the number of measurements needed. Traditional CS techniques focused on undersampling situations (i.e. with measurement rate $\alpha<1$ ). Here, results will be obtained for $\alpha \gg 1$ as well.

\subsection{ONLINE LEARNING}

As before, consider a signal $\boldsymbol{x}_{0}=\left(x_{0, i}\right) \in \mathbb{R}^{N}$ generated by distribution $\phi_{0}\left(\boldsymbol{x}_{0}\right)$. Suppose we generate a sequence of measurement vectors $\mathbf{A}^{\mu}=\left(A_{i}^{\mu}\right) \in \mathbb{R}^{N}$ and we use them to sequentially measure $\boldsymbol{x}_{0}$ through the inner products

$$
u_{0}^{\mu}=\mathbf{A}^{\mu} \cdot \boldsymbol{x}_{0} .
$$

Each projection pass through a (possibly noisy) channel, so that what is actually available is the measurement value $y^{\mu}$, which might be a continuous or discrete variable sampled independently from the conditional distribution

$$
y^{\mu} \sim P\left(y^{\mu} \mid u_{0}^{\mu}\right)
$$

After $t$ measurements, we will have had accumulated $t$ data $D^{t}=\left\{\left(\mathbf{A}^{1}, y^{1}\right),\left(\mathbf{A}^{2}, y^{2}\right)\right.$, $\left.\ldots,\left(\mathbf{A}^{t}, y^{t}\right)\right\}$. The main goal here then is to accurately recover $\boldsymbol{x}_{0}$ based on the best estimate $\phi(x)$ of the generating distribution $\phi_{0}(x)$ and on the knowledge of $D^{t}$ and the functional form and $P(y \mid u)$ in an online manner. 
Bayes' rule asserts that the posterior probability density of $x$, given all the data $D^{t}$ is

$$
P\left(x \mid D^{t}\right)=\frac{P\left(D^{t} \mid x\right) \phi(x)}{\int d x^{\prime} P\left(D^{t} \mid x^{\prime}\right) \phi\left(x^{\prime}\right)} .
$$

This formula provides a surprisingly simple and direct way to deal with the addition of a new datum $\left\{\mathbf{A}^{t+1}, y^{t+1}\right\}$ to $D^{t}$. With the assumption that all measurements are independent, the likelihood can be factorized according to

$$
\begin{aligned}
P\left(D^{t+1} \mid x\right) & =\prod_{\mu=1}^{t+1} P\left(\left\{y^{\mu}, \mathbf{A}^{\mu}\right\} \mid x\right) \\
& =P\left(\left\{y^{t+1}, \mathbf{A}^{t+1}\right\} \mid x\right) \prod_{\mu=1}^{t} P\left(\left\{y^{\mu}, \mathbf{A}^{\mu}\right\} \mid x\right) \\
& \equiv P\left(\left\{y^{t+1}, \mathbf{A}^{t+1}\right\} \mid x\right) P\left(D^{t} \mid x\right) .
\end{aligned}
$$

Thus, the information gathered from a new measurement produces a quantifiable change in the prior - indeed, the updated prior is the same as the one before the measurement times the factor $P\left(\left\{y^{t+1}, \mathbf{A}^{t+1}\right\} \mid x\right)$. The posterior distribution is updated accordingly with every new data point that is observed:

$$
P\left(x \mid D^{t+1}\right) \propto P\left(\left\{y^{t+1}, \mathbf{A}^{t+1}\right\} \mid x\right) P\left(x \mid D^{t}\right) .
$$

This expression already corresponds to an online update, but depending on the characteristics of $P(\{y, A\} \mid x)$ and $P(x \mid D)$, calculations might become unfeasible. The basic idea to transform the proto-update expression (3.5) into a full-fledged online algorithm is to replace the posterior distribution $P\left(x \mid D^{t}\right)$ by a parametric distribution $P\left(x ; \boldsymbol{\theta}^{t}\right)$, in which $\boldsymbol{\theta}^{t}=\left(\theta_{i}^{t}\right)$ is a set of parameters which must be updated with every measurement [96]. The parametric family of $P\left(x ; \boldsymbol{\theta}^{t}\right)$ must be chosen in such a way that loss of information in the approximation step is the smallest possible.

Let us define the Bayesian online algorithm as a repetition of two steps, namely, the update step, and the project step, both of which occur after every new measurement:

Update Given a new datum $\left\{\mathbf{A}^{t+1}, y^{t+1}\right\}$, use Bayes' rule to update the previous posterior $P\left(x \mid \boldsymbol{\theta}^{t}\right)$ :

$$
P\left(x \mid\left\{y^{t+1}, \mathbf{A}^{t+1}\right\} ; \boldsymbol{\theta}^{t}\right)=\frac{P\left(\left\{y^{t+1}, \mathbf{A}^{t+1}\right\} \mid x\right) P\left(x ; \boldsymbol{\theta}^{t}\right)}{\int d x^{\prime} P\left(\left\{y^{t+1}, \mathbf{A}^{t+1}\right\} \mid x^{\prime}\right) P\left(x^{\prime} ; \boldsymbol{\theta}^{t}\right)}
$$

Projection The new posterior is projected into a distribution of the chosen para- 
metric family:

$$
P\left(x \mid \boldsymbol{\theta}^{t+1}\right) \simeq P\left(x \mid\left\{y^{t+1}, \mathbf{A}^{t+1}\right\} ; \boldsymbol{\theta}^{t}\right) .
$$

The projection step is necessary because, with the exception of very specific cases, after the update step the new posterior will usually not belong to the family $P\left(x ; \boldsymbol{\theta}^{t}\right)$. After these two steps, an estimate $\hat{\boldsymbol{x}}$ of the signal $\boldsymbol{x}_{0}$ can be inferred from $P\left(x ; \boldsymbol{\theta}^{t+1}\right)$.

As mentioned, in the projection step it is interesting to perform the best approximation possible, so that most information is conserved. Finding the distribution that best approximates the posterior necessitates the definition of a metric of similarity, and below we present the most commonly used: the Kullback-Leibler divergence (or simply, KL-divergence). For any two continuous distributions $g(x)$ and $h(x)$, the KL-divergence is defined as $^{1}$

$$
D_{K L}(g \| h)=\int_{-\infty}^{+\infty} d x h(x) \ln \frac{h(x)}{g(x)}
$$

The KL-divergence is also called relative entropy, since it can also be trivially written $D_{K L}(g \| h)=S[g, h]-S[h]$. Here, $S[g, h]=-\int d x g(x) \ln h(x)$ is the cross-entropy of distributions $g(x)$ and $h(x)$ and $S[h]=-\int d x h(x) \ln h(x)$ is the entropy of $h(x)$. Being a non-symmetric quantity $\left(D_{K L}(g \| h) \neq D_{K L}(h \| g)\right.$ in general $)$, the KLdivergence might not be directly understood as a simple metric, but as a premetric. In fact, it does not obey the triangle inequality [27]. But an interesting property makes it suitable for comparing distributions:

$$
D_{K L}(g \| h) \geq 0
$$

always, with equality only true when $g$ and $h$ are exactly the same [4].

When dealing with distributions from the exponential family [3],

$$
P(x ; \boldsymbol{\theta})=\frac{1}{Z(\boldsymbol{\theta})} \exp \left[-\sum_{k} \theta_{k} f_{k}(x)\right],
$$

where $Z(\boldsymbol{\theta})=\int d x \exp \left[-\sum_{k} \theta_{k} f_{k}(x)\right]$ is a normalization factor, minimization of $D_{K L}(g \| P(x ; \boldsymbol{\theta}))$ with respect to the parameters $\theta_{k}$ for whatever distribution $g(x)$

\footnotetext{
${ }^{1}$ For discrete distributions, the integral is substituted by a summation:

$$
D_{K L}(g \| h)=\sum_{x^{\prime}} h\left(x^{\prime}\right) \ln \left[h\left(x^{\prime}\right) / g\left(x^{\prime}\right)\right] .
$$
}


can be easily calculated:

$$
\begin{aligned}
D_{K L}(g \| P(x ; \boldsymbol{\theta})) & =\int d x g(x) \ln \frac{g(x)}{\exp \left[-\sum_{k} \theta_{k} f_{k}(x)\right] / Z(\boldsymbol{\theta})} \\
& =S[g]+\ln Z(\boldsymbol{\theta})-\int d x g(x) \sum_{k} \theta_{k} f_{k}(x) .
\end{aligned}
$$

Since $\left(\partial / \partial \theta_{k}\right) \ln Z(\theta)=(1 / Z(\theta)) \int d x f_{k}(x) \exp \left[-\sum_{k} \theta_{k} f_{k}(x)\right]=\int d x f_{k}(x) P(x ; \theta)$, it is straightfoward to see that

$$
\frac{\partial}{\partial \theta_{k}} D_{K L}(g \| P(x ; \boldsymbol{\theta}))=0 \quad \Rightarrow \quad\left\langle f_{k}(x)\right\rangle_{g}=\left\langle f_{k}(x)\right\rangle_{P(x \mid \boldsymbol{\theta})} .
$$

In other words, for exponential distributions the minimization of the KL-divergence implies the adjustment of the parameters $\theta_{k}$ so that the moments $\left\langle f_{k}(x)\right\rangle$ match for both distributions.

Now consider the choice of multivariate Gaussian distributions $P\left(x ; \boldsymbol{\theta}^{t}\right)$ in the Bayesian online learning algorithm. Let us perform the projection step by minimization of the KL-divergence between the posterior distribution after a new measurement and some distribution of the chosen format. Equation (3.13) means that the projection step is equivalent to finding parameters $\boldsymbol{\theta}$ such that the means $\boldsymbol{m}$ and all covariances $C_{i j}$ remain the same before and after the projection, i.e.

$$
\begin{aligned}
m_{i}^{t+1} & =\frac{\int d \boldsymbol{x} x_{i} P\left(\left\{y^{t+1}, \mathbf{A}^{t+1}\right\} \mid x\right) P\left(x ; \boldsymbol{\theta}^{t}\right)}{\int d x^{\prime} P\left(\left\{y^{t+1}, \mathbf{A}^{t+1}\right\} \mid x^{\prime}\right) P\left(x^{\prime} ; \boldsymbol{\theta}^{t}\right)}, \\
C_{i j}^{t+1} & =\frac{\int d \boldsymbol{x} x_{i} x_{j} P\left(\left\{y^{t+1}, \mathbf{A}^{t+1}\right\} \mid x\right) P\left(x ; \boldsymbol{\theta}^{t}\right)}{\int d x^{\prime} P\left(\left\{y^{t+1}, \mathbf{A}^{t+1}\right\} \mid x^{\prime}\right) P\left(x^{\prime} ; \boldsymbol{\theta}^{t}\right)}-m_{i}^{t+1} m_{j}^{t+1} .
\end{aligned}
$$

As an example, imagine the minimalist scenario where the scalar $x_{0} \sim \mathcal{N}(1,2)$ is directly observed in the presence of additive Gaussian noise, $y^{\mu}=x+\xi^{\mu}$, where $\xi^{\mu} \sim \mathcal{N}\left(0, \sigma_{n}^{2}\right)$ (Figure 3.1). We choose the parametric distribution $P(x ; \boldsymbol{\theta})$ to be Gaussian so that equations (3.14) and (3.15) correctly describe the projection step. What happens exactly with the arrival of a new measurement $y^{t+1}$ ? Before the measurement, the approximate distribution for $x$ is the univariate Gaussian with mean $m^{t}$ and variance $v^{t}$,

$$
P\left(x ; m^{t}, v^{t}\right)=\frac{1}{\sqrt{2 \pi v^{t}}} \exp \left[-\frac{1}{2 v^{t}}\left(x-m^{t}\right)^{2}\right] \propto \exp -\frac{1}{2}\left[\frac{1}{v^{t}} x^{2}-2 \frac{m^{t}}{v^{t}} x\right] .
$$

With the information obtained with $y^{t+1}$, the update step gives us the posterior 


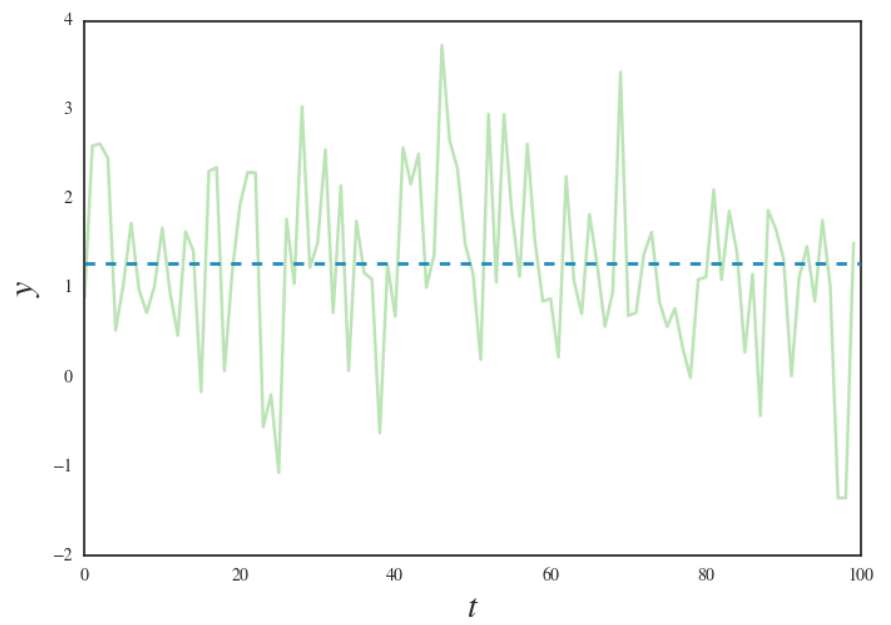

Figure 3.1: Measurements $y \sim \mathcal{N}\left(x_{0}, \sigma_{n}^{2}\right)$. Blue dashed line: $x_{0} \simeq 1.28$.

distribution the posterior distribution

$$
\begin{aligned}
P\left(x \mid D^{t+1}\right) & \propto \exp \left[-\frac{1}{2 \sigma_{n}^{2}}\left(y^{t+1}-x\right)^{2}\right] \exp \left[-\frac{1}{2 v^{t}}\left(x-m^{t}\right)^{2}\right] \\
& \propto \exp -\frac{1}{2}\left[\left(\frac{1}{\sigma_{n}^{2}}+\frac{1}{v^{t}}\right) x^{2}-2\left(\frac{y^{t+1}}{\sigma_{n}^{2}}+\frac{m^{t}}{v^{t}}\right) x\right]
\end{aligned}
$$

Now the projection step consists in matching the moments of both $P\left(x \mid D^{t+1}\right)$ and $P\left(x \mid m^{t+1}, v^{t+1}\right)$. Making use of the expressions (3.16) and (3.18), this is done by solving the system of equations

$$
\left\{\begin{array}{l}
\frac{1}{v^{t+1}}=\frac{1}{\sigma_{n}^{2}}+\frac{1}{v^{t}} \\
\frac{m^{t+1}}{v^{t+1}}=\frac{y^{t+1}}{\sigma_{n}^{2}}+\frac{m^{t}}{v^{t}}
\end{array}\right.
$$

resulting in the following expressions:

$$
\begin{aligned}
m^{t+1} & =m^{t}+v^{t} \frac{y^{t+1}-m^{t}}{v^{t}+\sigma_{n}^{2}}, \\
v^{t+1} & =v^{t}-\frac{\left(v^{t}\right)^{2}}{v^{t}+\sigma_{n}^{2}} .
\end{aligned}
$$

In Figure (3.2) it is possible to visualize this online update. With more and more measurements, the estimate $m^{t}$ approaches the true value $x_{0}$, even when the prior $P\left(x ; m^{0}, v^{0}\right)=\mathcal{N}(0,4)$ does not correspond to the true generating signal distribution $\phi_{0}\left(x_{0}\right)$ (Figure 3.2c).

Besides being obvious choices for doctorate thesis examples, Gaussian distri- 


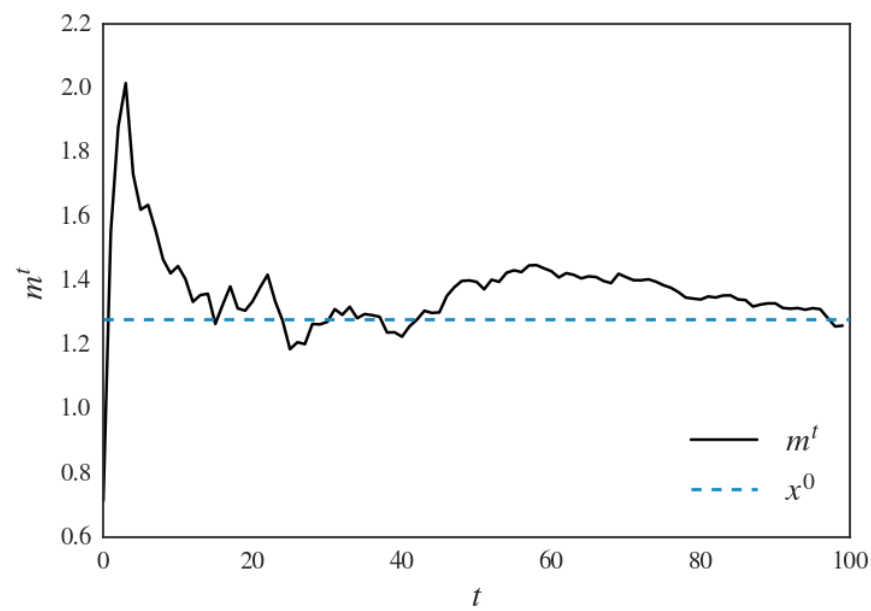

(a)

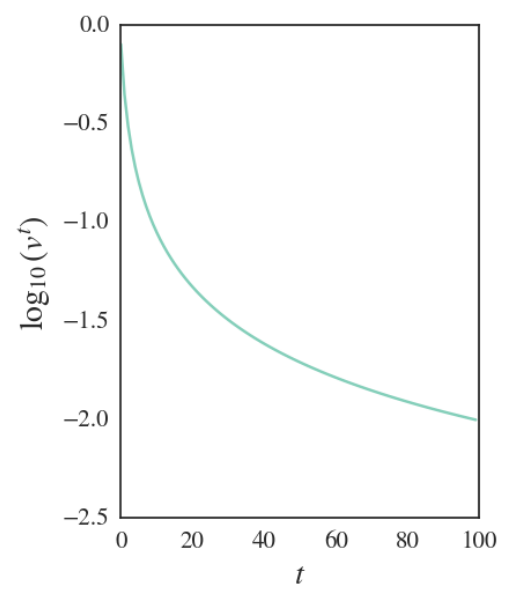

(b)

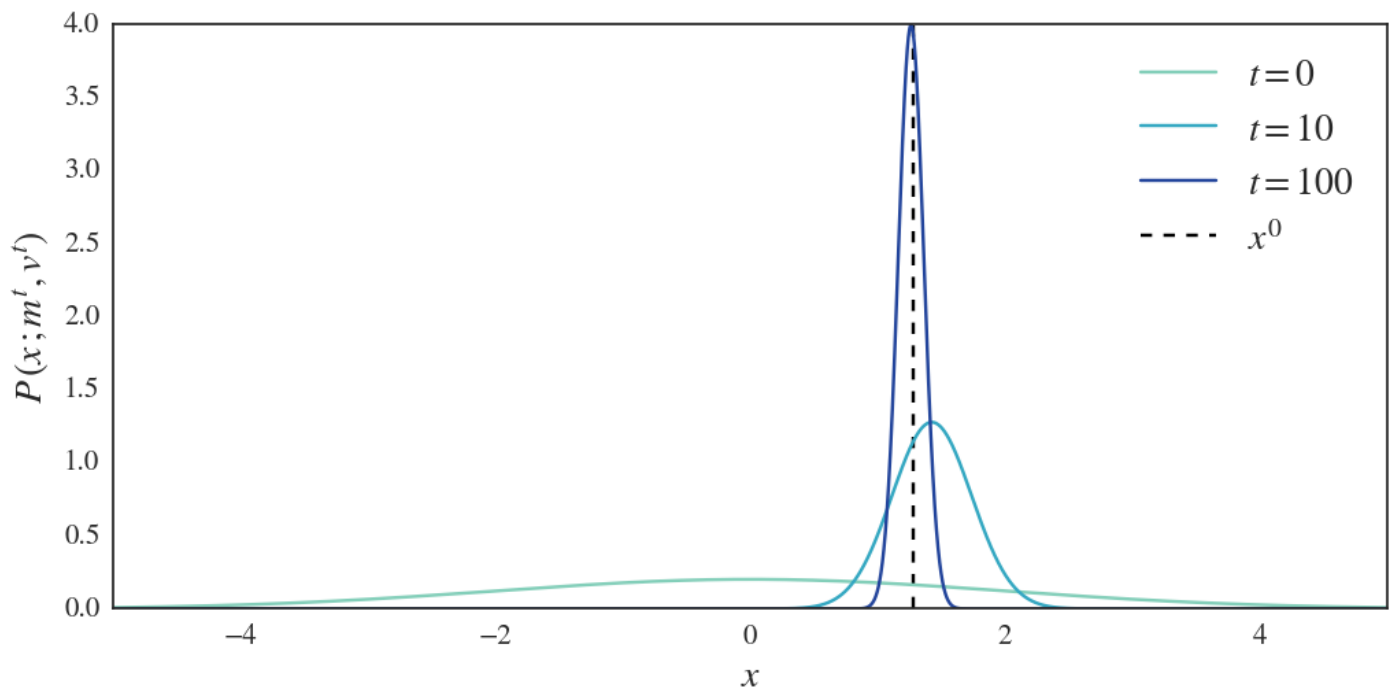

(c)

Figure 3.2: One-dimensional Bayesian online learning. (a) Evolution of the signal's estimate $m^{t}$; (b) Evolution of the signal's estimate variance $v^{t}$; (c) Evolution of the parameterized (or projected) distribution of the signal's estimate. Signal generating distribution: $x_{0} \sim \mathcal{N}(1,1)$. Prior distribution: $P\left(x ; m^{0}, v^{0}\right)=\mathcal{N}(0,4)$. Note the collapse of the Gaussian distribution around the true value $x_{0}$ (black, dashed) for large $t$, even when the prior is incorrect.

butions are of special interest for several other reasons. Notice that for the standard scenario of linear measurements with additive noise $y^{\mu}=\mathbf{A}^{\mu} \cdot \boldsymbol{x}+\xi^{\mu}$, with $\xi^{\mu} \sim \mathcal{N}\left(0, \sigma_{n}^{2}\right)$, Bayes' theorem (3.3) can be rewritten

$$
P\left(x \mid\left\{y^{\mu}, \mathbf{A}^{\mu}\right\}_{\mu=1}^{t}\right) \propto \exp \left[-\frac{1}{2 \sigma_{n}^{2}} \sum_{\mu=1}^{t}\left(y^{\mu}-\mathbf{A}^{\mu} \cdot \boldsymbol{x}\right)^{2}+\log \phi(x)\right] .
$$

This means that as long as $\phi(x)$ is well behaved, for large $t$ the summation term 
becomes much larger than the constant logarithm, i.e. the likelihood dominates the prior, and $P\left(x \mid D^{t}\right)$ approaches a Gaussian distribution. In fact, this result is even more general — for any likelihood $P(y \mid x)$ the posterior can be written $P\left(x \mid\left\{y^{\mu}, \mathbf{A}^{\mu}\right\}_{\mu=1}^{t}\right) \propto \exp \left[\sum_{\mu=1}^{t} \log P\left(y^{\mu} \mid \mathbf{A}^{\mu} \cdot \boldsymbol{x}\right)+\log \phi(x)\right]$, which is guaranteed by the law of large numbers to asymptotically approach a Gaussian distribution. Another intuitive way of looking at this is to consider that for smooth distributions $P(\{y, \mathbf{A}\} \mid x)$ and $P(x ; \boldsymbol{\theta})$, when the number of examples is sufficiently large the posterior distribution is strongly concentrated around its maximum. This allows us to approximate $P\left(x ; \boldsymbol{\theta}^{t}\right)$ for a multivariate Gaussian distribution. ${ }^{2}$ Unfortunately, in Compressed Sensing the sparse generating distribution $\phi_{0}(\boldsymbol{x})$ is not well-behaved so the Gaussian approximation is not ideal even when $t \rightarrow \infty$. The next section introduces modifications to the theory above, producing a new algorithm with a specific application to Compressed Sensing.

\subsection{Bayesian Online Compressed Sensing Algo- RITHM}

In Compressed Sensing we are dealing with signals that possess an inherent and crucial characteristic: they are made up of many null components. As it has already been noted above, this means that we can model each component of the signal as being generated by the distribution $\phi(x)=(1-\rho) \delta(x)+\rho g(x)$. The singularity due to the $\delta(x)$ factor makes any convergence of the posterior to a Gaussian distribution in the online setting impossible - it does not matter how many elements you sum, there will always be a $\delta(x)$ factor in there. This means that the approach described at the end of the previous section, which consisted of using a Gaussian approximation for the posterior distribution is not necessarily efficient in a online Compressed Sensing scenario - in fact, it is far from optimal. But as will be shown, the introduction of an alternative projection step is enough to adapt the Bayesian online framework to learn sparse signals from random projections.

\footnotetext{
${ }^{2}$ In fact, it is possible to write the posterior distribution as a Gibbs' distribution $P\left(x \mid D^{t}\right) \propto$ $\exp [-\beta \mathcal{H}(x)]$ and expand a Taylor series around the maximum of the Hamiltonian:

$$
\mathcal{H}(x)=\mathcal{H}\left(x^{*}\right)+\frac{1}{2} \sum_{j k}\left(x_{j}-x_{k}\right)\left(\frac{\partial^{2}}{\partial x_{j} \partial x_{k}} \mathcal{H}\left(x^{*}\right)\right)\left(x_{k}-x_{k}^{*}\right)+\ldots
$$

By taking only the first two terms of the expansion, one arrives at the Gaussian approximation

$$
P\left(x \mid D^{t}\right) \propto \exp \left[-\frac{1}{2}\left(x-x_{t}^{*}\right) \underline{C}_{t}^{-1}\left(x-x_{t}^{*}\right)\right] \text {, with } C_{t, j k}^{-1}=\frac{\partial^{2}}{\partial x_{j} \partial x_{k}} \mathcal{H}\left(x^{*}\right) .
$$
}


This can be achieved with the introduction of a mean field approximation into the projection step, along with explicitly maintaining the sparse prior $\phi(x)=\phi_{0}(x)$ :

$$
P\left(\boldsymbol{x} \mid D^{t}\right) \simeq \widetilde{P}\left(\boldsymbol{x} \mid D^{t}\right):=\prod_{i=1}^{N}\left(\frac{e^{-a_{i}^{t} x_{i}^{2} / 2+h_{i}^{t} x_{i}} \phi\left(x_{i}\right)}{Z\left(a_{i}^{t}, h_{i}^{t}\right)}\right),
$$

where $Z\left(a_{i}^{t}, h_{i}^{t}\right)=\int d x_{i} \exp \left(-a_{i}^{t} x_{i}^{2} / 2+h_{i}^{t} x_{i}\right) \phi\left(x_{i}\right)$ and $\widetilde{P}\left(\boldsymbol{x} \mid D^{t}\right) \equiv \widetilde{P}\left(\boldsymbol{x} ;\left\{\left(a_{i}^{t}, h_{i}^{t}\right)\right\}_{i=1}^{N}\right)$. Making the prior ever-present in the inference process allows for the singularity to be properly modelled. The mean field approximation ${ }^{3}$, as a technical device, eliminates a considerable fraction of the variables that would otherwise be present in a full Gaussian distribution $\widetilde{P}\left(D^{t} \mid x\right) \propto \exp -(1 / 2) \sum_{i>j} x_{i} a_{i j}^{t} x_{j}+\sum_{i} h_{i}^{t} x_{i}$. Thus, it makes the reconstruction algorithm computationally cheaper both in processing power and memory use. ${ }^{4}$ In addition, the diagonalization of $\widetilde{P}\left(x \mid D^{t}\right)$ comes with a nice collateral effect: while in general the calculation of the mmse estimator $\boldsymbol{m}^{t}=$ $\int d \boldsymbol{x} \boldsymbol{x} P\left(\boldsymbol{x} \mid D^{t}\right)$ can be difficult, in the current setup the means and variances of each component $x_{i}$ are simply computed as

$$
\begin{aligned}
m_{i}^{t} & =\left(\partial / \partial h_{i}^{t}\right) \ln Z\left(a_{i}^{t}, h_{i}^{t}\right), \\
v_{i}^{t} & =\left(\partial^{2} /\left(\partial h_{i}^{t}\right)^{2}\right) \ln Z\left(a_{i}^{t}, h_{i}^{t}\right),
\end{aligned}
$$

respectively.

In summary, from (3.2) and (3.23), the projection and update steps in the Online CS algorithm can be summarized as below:

Update After any measurement, update the posterior distribution with the new information:

$$
P\left(\boldsymbol{x} \mid D^{t}\right) \propto P\left(y^{t} \mid \boldsymbol{A}^{t} \cdot \boldsymbol{x}\right) \widetilde{P}\left(\boldsymbol{x} \mid D^{t-1}\right)
$$

Projection Approximate the posterior distribution by a distribution like (3.23):

$$
P\left(\boldsymbol{x} \mid D^{t}\right) \simeq \widetilde{P}\left(\boldsymbol{x} \mid D^{t}\right)
$$

Figure 3.3 provides a visual explanation of the process. A new measurement might remove the posterior from the predefined space of distributions (3.23), so the projection step return it (the posterior) to a tractable form. As before, this approximation must be done with care. In the next paragraphs we will be concerned with describing in detail a method for getting an appropriate projected distribution.

\footnotetext{
${ }^{3}$ Mean field approximations have already been introduced in CS scenarios in [40], but not in an online fashion.

${ }^{4}$ It is clear from (3.23) that the posterior distribution can be entirely described by the set of $2 N$ time-dependent natural parameters $\left(a_{i}^{t}, h_{i}^{t}\right)$.
} 


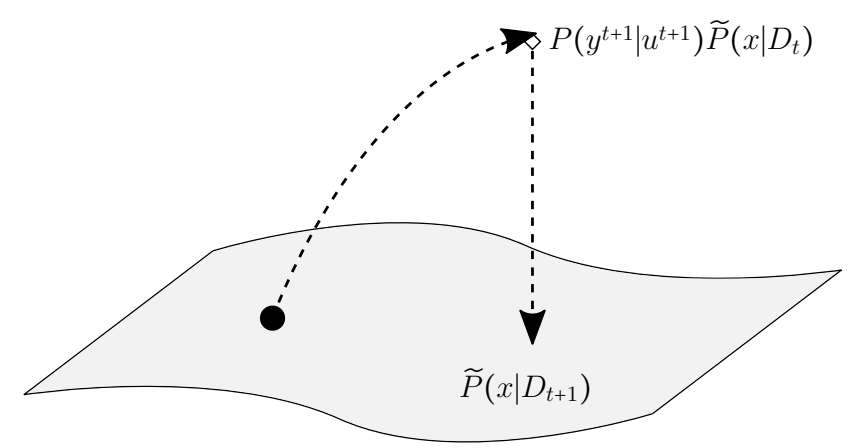

Figure 3.3: Update and projection steps in Bayesian online learning. In the update step, information from the new measurements might remove the posterior distribution from the "projection manifold". The projection step returns the posterior to the manifold by finding the best approximation in the parameterized distribution family.

Imagine $t$ measurements have already been made so that the natural parameters $\left\{\left(a_{i}^{t}, h_{i}^{t}\right)\right\}$ are already given. At this stage, the estimate for the posterior distribution is exactly as (3.23). At $t+1$, another random projection $y^{t} \sim P\left(y^{t} \mid \boldsymbol{A}^{t} \cdot \boldsymbol{x}\right)$ is obtained, so that an update to our knowledge (represented by the posterior estimate) is possible. Therefore, according to the update rule, after the most recent measurement we have

$$
P\left(x \mid D^{t+1}\right) \propto P\left(y^{t+1} \mid \mathbf{A}^{t+1} \cdot \boldsymbol{x}\right) \prod_{i}\left(e^{-a_{i}^{t} x_{i}^{2} / 2+h_{i}^{t} x_{i}} \phi\left(x_{i}\right)\right) .
$$

Approximation of this expression to a distribution like (3.23) will be done through comparison of their marginals $P_{i}\left(x_{i} \mid D^{t+1}\right)=\int d \boldsymbol{x}_{\backslash i} P\left(\boldsymbol{x} \mid D^{t+1}\right)$ and $\widetilde{P}_{i}\left(x_{i} \mid D^{t+1}\right)=$ $\int d \boldsymbol{x}_{\backslash i} \widetilde{P}\left(\boldsymbol{x} \mid D^{t+1}\right)$. Let us first marginalize (3.28) with respect to index $i$ :

$$
\begin{aligned}
& P_{i}\left(x_{i} \mid D^{t+1}\right) \propto e^{-a_{i}^{t} x_{i}^{2} / 2+h_{i}^{t} x_{i}} \phi\left(x_{i}\right) \times \\
& \times \int\left(\prod_{j \neq i} d x_{j} e^{-a_{j}^{t} x_{j}^{2} / 2+h_{j}^{t} x_{j}} \phi\left(x_{j}\right)\right) P\left(y^{t+1} \mid \mathbf{A}^{t+1} \cdot \boldsymbol{x}\right) .
\end{aligned}
$$

As all components $A_{j}^{t+1}$ of our measurement apparatus are normal random variables of zero mean and variance $N^{-1}$, any weighted sum $\sum_{k} w_{k} A_{k}^{t+1}$ with weights $w_{k} \in \mathbb{R}$ should also be a normal random variable. This means we can approximate the summation $u_{\backslash i}^{t+1} \equiv \sum_{j \neq i} A_{j}^{t+1} x_{j}$ by a Gaussian random variable with mean $\Delta_{\backslash i}^{t+1}=$ $\sum_{j \neq i} A_{j}^{t+1} m_{j}^{t}$ and variance $\chi_{\backslash i}^{t+1}=\sum_{j \neq i}\left(A_{j}^{t+1}\right)^{2} v_{j}^{t} \simeq N^{-1} \sum_{j \neq i} v_{j}^{t}$. In other words,

$$
u_{\backslash i}^{t+1} \simeq \Delta_{\backslash i}^{t+1}+\sqrt{\chi_{\backslash i}^{t+1}} z
$$

where $z$ is a standard normal random variable $z \sim \mathcal{N}(0,1)$. This approximation greatly simplifies the multidimensional integration on all $x_{j}$ above, turning it into a simple univariate integration on $z$. That is, we can replace the measure 
$\prod_{j \neq i} d x_{j} \exp \left(-a_{j}^{t} x_{j}^{2} / 2+h_{j}^{t} x_{j}\right) \phi\left(x_{j}\right)$ for the Gaussian measure $\mathcal{D} z \equiv d z \exp \left(-z^{2} / 2\right) / \sqrt{2 \pi}$. With $\boldsymbol{A}^{t+1} \cdot \boldsymbol{x} \simeq A_{i}^{t+1} x_{i}+u_{\backslash i}^{t+1}$, the marginal distribution of $x_{i}$ (3.29) becomes then

$$
P_{i}\left(x_{i} \mid D^{t+1}\right) \propto e^{-a_{i}^{t} x_{i}^{2} / 2+h_{i}^{t} x_{i}} \phi\left(x_{i}\right) \int \mathcal{D} z P\left(y^{t+1} \mid A_{i}^{t+1} x_{i}+\Delta_{\backslash i}^{t+1}+\sqrt{\chi_{\backslash i}^{t+1}} z\right)
$$

In addition, the smallness of the factor $\left|A_{i}^{t+1} x_{i}\right| \sim O\left(N^{-1 / 2}\right) \ll 1$, allows us to expand $\ln \int \mathcal{D} z P\left(y^{t+1} \mid A_{i}^{t+1} x_{i}+\Delta_{\backslash i}^{t+1}+\sqrt{\chi_{\backslash i}^{t+1}} z\right)$ up to second order in $A_{i}^{t+1} x_{i}$ as

$$
\begin{aligned}
\ln \int \mathcal{D} z P\left(y^{t+1} \mid A_{i}^{t+1} x_{i}+\Delta_{\backslash i}^{t+1}\right. & \left.+\sqrt{\chi_{\backslash i}^{t+1}} z\right) \\
\simeq & \ln \int \mathcal{D} z P\left(y^{t+1} \mid \Delta_{\backslash i}^{t+1}+\sqrt{\chi_{\backslash i}^{t+1}} z\right) \\
& +A_{i}^{t+1} x_{i} \frac{\partial}{\partial \Delta_{\backslash i}^{t+1}}\left(\ln \int \mathcal{D} z P\left(y^{t+1} \mid \Delta_{\backslash i}^{t+1}+\sqrt{\chi_{\backslash i}^{t+1}} z\right)\right) \\
& +\frac{\left(A_{i}^{t+1}\right)^{2} x_{i}^{2}}{2} \frac{\partial^{2}}{\partial\left(\Delta_{\backslash i}^{t+1}\right)^{2}}\left(\ln \int \mathcal{D} z P\left(y^{t+1} \mid \Delta_{\backslash i}^{t+1}+\sqrt{\chi_{\backslash i}^{t+1}} z\right)\right)
\end{aligned}
$$

with sufficient accuracy. Let us define the function $\Omega(\Delta, \chi ; y):=\ln \int \mathcal{D} z P(y \mid \Delta+$ $\sqrt{\chi} z)$ and its two first derivatives $\Omega^{\prime}(\Delta, \chi ; y):=(\partial / \partial \Delta) \Omega(\Delta, \chi ; y)$ and $\Omega^{\prime \prime}(\Delta, \chi ; y):=$ $\left(\partial^{2} / \partial \Delta^{2}\right) \Omega(\Delta, \chi ; y)$ and use the approximation above to rewrite the marginal (3.31) as

$$
\begin{aligned}
P_{i}\left(x_{i} \mid D^{t+1}\right) \propto \exp \left(-a_{i}^{t} x_{i}^{2} / 2+h_{i}^{t} x_{i}\right) \phi\left(x_{i}\right) \exp [ & \Omega\left(\Delta_{\backslash i}^{t+1}, \chi_{\backslash i}^{t+1} ; y^{t+1}\right) \\
& +A_{i}^{t+1} x_{i} \Omega^{\prime}\left(\Delta_{\backslash i}^{t+1}, \chi_{\backslash i}^{t+1} ; y^{t+1}\right) \\
& \left.+\frac{\left(A_{i}^{t+1}\right)^{2} x_{i}^{2}}{2} \Omega^{\prime \prime}\left(\Delta_{\backslash i}^{t+1}, \chi_{\backslash i}^{t+1} ; y^{t+1}\right)\right] .
\end{aligned}
$$

This expression can be directly compared with the marginal distribution of the R.H.S. of equation (3.23), whose very straightforward calculation results in

$$
\widetilde{P}_{i}\left(x_{i} \mid D^{t+1}\right)=\int d \boldsymbol{x}_{\backslash i} \prod_{i=1}^{N}\left(\frac{e^{-a_{i}^{t} x_{i}^{2} / 2+h_{i}^{t} x_{i}} \phi\left(x_{i}\right)}{Z\left(a_{i}^{t}, h_{i}^{t}\right)}\right) \propto e^{-a_{i}^{t+1} x_{i}^{2} / 2+h_{i}^{t+1} x_{i}} \phi\left(x_{i}\right) .
$$

Now the actual projection step consists in equating the right-hand sides of equations (3.33) and (3.34):

$$
P_{i}\left(x_{i} \mid D^{t+1}\right) \simeq \widetilde{P}_{i}\left(x_{i} \mid D^{t+1}\right) .
$$

Update rules for the parameters $\left\{\left(a_{i}^{t}, h_{i}^{t}\right)\right\}$, i.e. rules that take the set $\left\{\left(a_{i}^{t}, h_{i}^{t}\right)\right\}$ to the set $\left\{\left(a_{i}^{t+1}, h_{i}^{t+1}\right)\right\}$ can be obtained by comparing the terms with the same powers 
of $x_{i}$ inside the exponentials in both sides of the equality. This results in

$$
\left\{\begin{aligned}
a_{i}^{t+1} & =a_{i}^{t}-\left(A_{i}^{t+1}\right)^{2} \frac{\partial^{2}}{\partial\left(\Delta_{\backslash i}^{t+1}\right)^{2}}\left(\ln \int \mathcal{D} z P\left(y^{t+1} \mid \Delta_{\backslash i}^{t+1}+\sqrt{\chi_{\backslash i}^{t+1}} z\right)\right) \\
h_{i}^{t+1} & =h_{i}^{t}+A_{i}^{t+1} \frac{\partial}{\partial \Delta_{\backslash i}^{t+1}}\left(\ln \int \mathcal{D} z P\left(y^{t+1} \mid \Delta_{\backslash i}^{t+1}+\sqrt{\chi_{\backslash i}^{t+1}} z\right)\right) .
\end{aligned}\right.
$$

As a last step in the calculation of the update rules, we note that the derivative terms - as functions of $\Delta_{\backslash i}^{t+1}$ and $\chi_{\backslash i}^{t+1}$, are actually functions of $\sum_{j \neq i} A_{j}^{t+1} x_{j}$. By means of a second Taylor expansion, it is possible to write the equations as functions of $\sum_{j} A_{j}^{t+1} x_{j}$ (index $i$ included):

$$
\begin{aligned}
f\left(\sum_{j \neq i} A_{j} x_{j}\right) & =f\left(\sum_{j} A_{j} x_{j}-A_{i} x_{i}\right) \\
& =f\left(\sum_{j} A_{j} x_{j}\right)-f^{\prime}\left(\sum_{j} A_{j} x_{j}\right)\left(A_{i} x_{i}\right)+O\left(A_{i}^{2}\right) .
\end{aligned}
$$

Therefore, up to second order on $A_{i}^{t+1}$, the complete update equations are ${ }^{5}$

$$
\left\{\begin{aligned}
a_{i}^{t+1}= & a_{i}^{t}-\left(A_{i}^{t+1}\right)^{2} \frac{\partial^{2}}{\partial\left(\Delta^{t+1}\right)^{2}}\left(\ln \int \mathcal{D} z P\left(y^{t+1} \mid \Delta^{t+1}+\sqrt{\chi^{t+1}} z\right)\right) \\
h_{i}^{t+1}= & h_{i}^{t}+A_{i}^{t+1} \frac{\partial}{\partial \Delta^{t+1}}\left(\ln \int \mathcal{D} z P\left(y^{t+1} \mid \Delta^{t+1}+\sqrt{\chi^{t+1}} z\right)\right) \\
& -m_{i}\left(A_{i}^{t+1}\right)^{2} \frac{\partial^{2}}{\partial\left(\Delta^{t+1}\right)^{2}}\left(\ln \int \mathcal{D} z P\left(y^{t+1} \mid \Delta^{t+1}+\sqrt{\chi^{t+1}} z\right)\right)
\end{aligned}\right.
$$

where $\Delta^{t} \equiv \sum_{i=1}^{N} A_{i}^{t} m_{i}^{t-1}$ and $\chi^{t} \equiv \sum_{i=1}^{N}\left(A_{i}^{t}\right)^{2} v_{i}^{t-1}$. These equations, just like (3.20) and (3.21) for the one-dimensional example in the previous section, summarize both the update and project steps. The iteration of these equations together with expressions (3.24) and (3.25) make up what we call the Bayesian Online CS Algorithm (Algorithm 1).

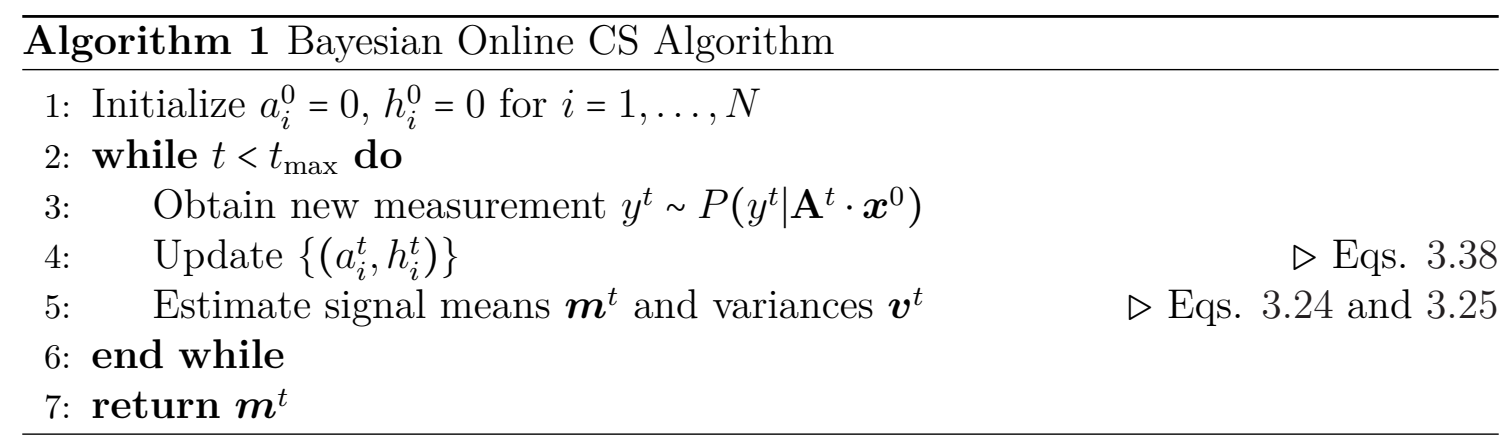

${ }^{5}$ Even though the third term on the right-hand side of the last expression is of order $O\left(N^{-1}\right)$ while the second term is of order $O\left(N^{-1 / 2}\right)$, in the former $\left(A_{i}^{t+1}\right)^{2}$ is always a positive number and its successive accumulation is substantive for $\alpha \sim O(1)$ (which means $t \sim O(N)$ ) so that it cannot be ignored in this scenario. 


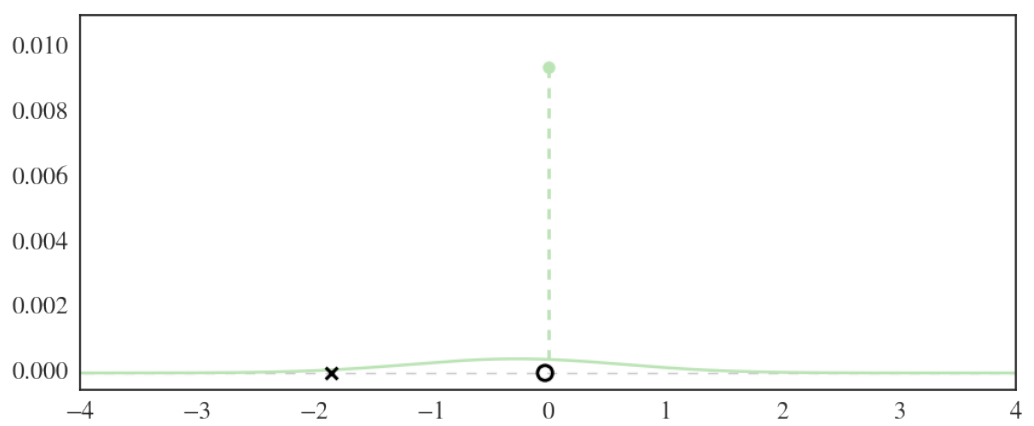

(a) $\alpha=0.05$

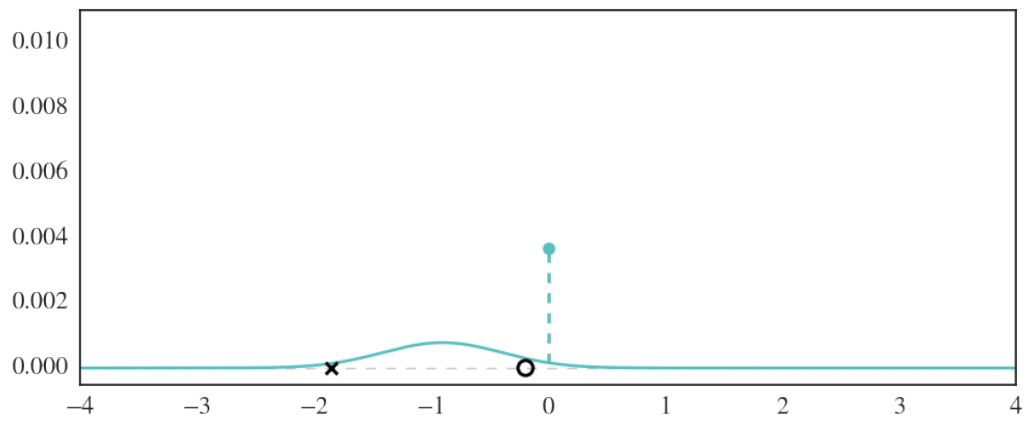

(b) $\alpha=0.4$

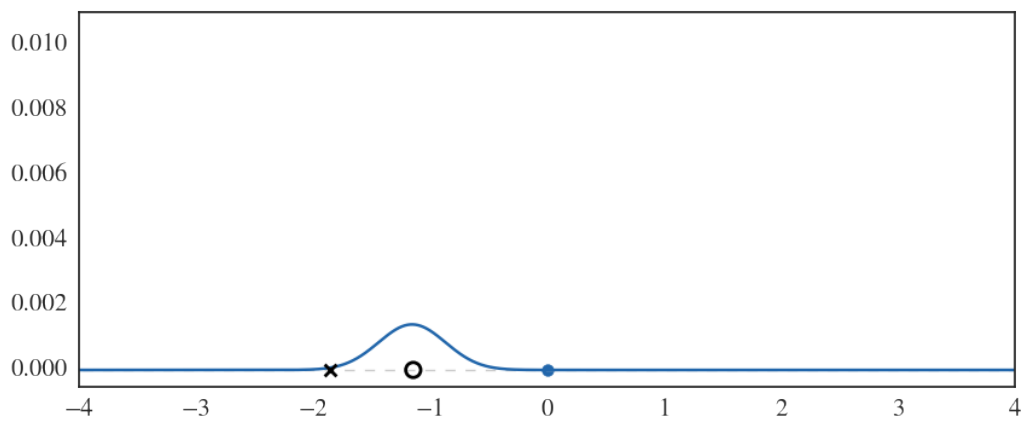

(c) $\alpha=1$

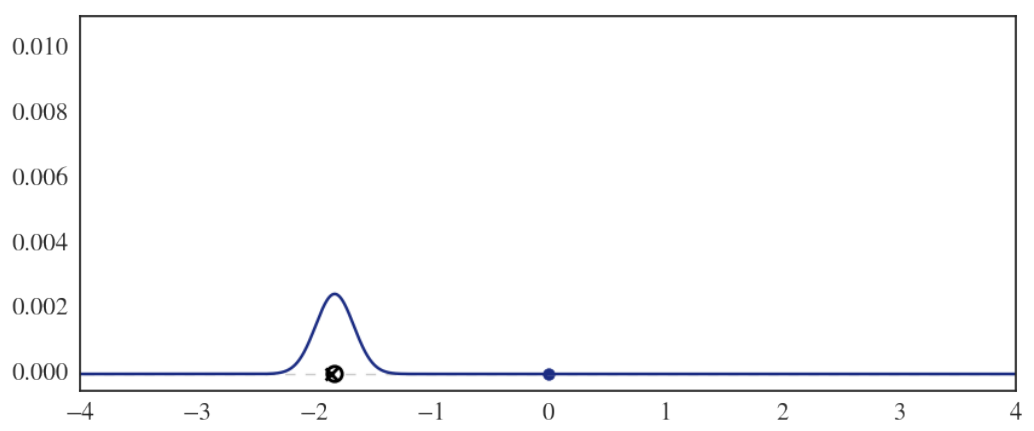

(d) $\alpha=1.5$

Figure 3.4: Evolution of the posterior distribution $\widetilde{P}_{i}\left(x_{i} \mid D^{t}\right)$ for a non-zero component of the signal. The vertical dashed line corresponds to the $\delta\left(x_{i}\right)$ factor in the prior distribution - the dot on its top is the probability $\widetilde{P}_{i}\left(x_{i}=0 \mid D^{t}\right)$. Cross: true value $x_{0, i}$; Circle: signal estimate (posterior mean) $m_{i}^{t}$. 


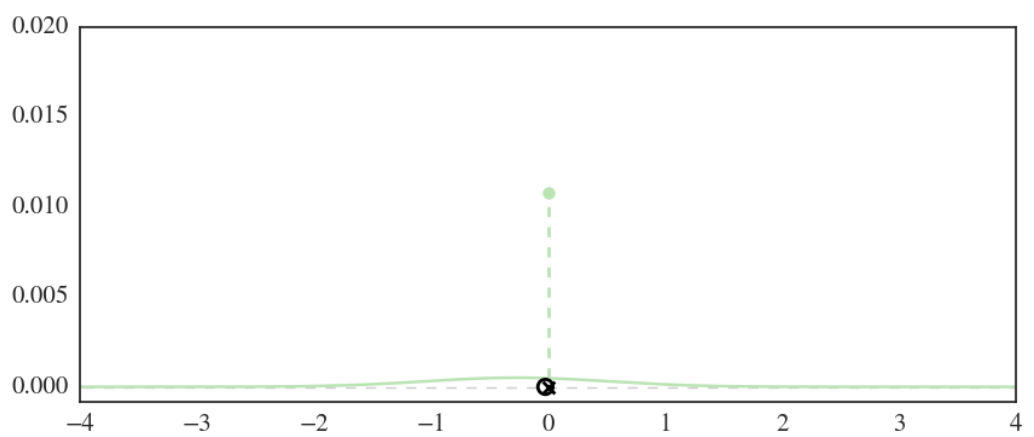

(a) $\alpha=0.05$

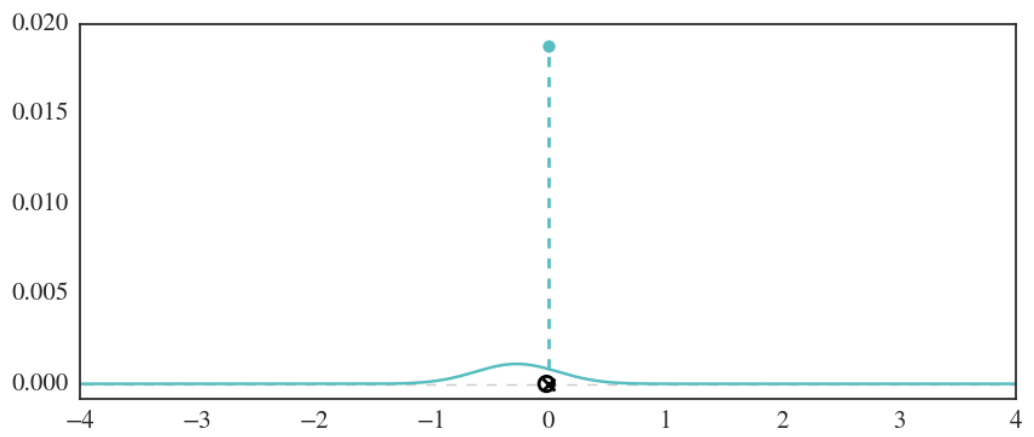

(b) $\alpha=0.4$

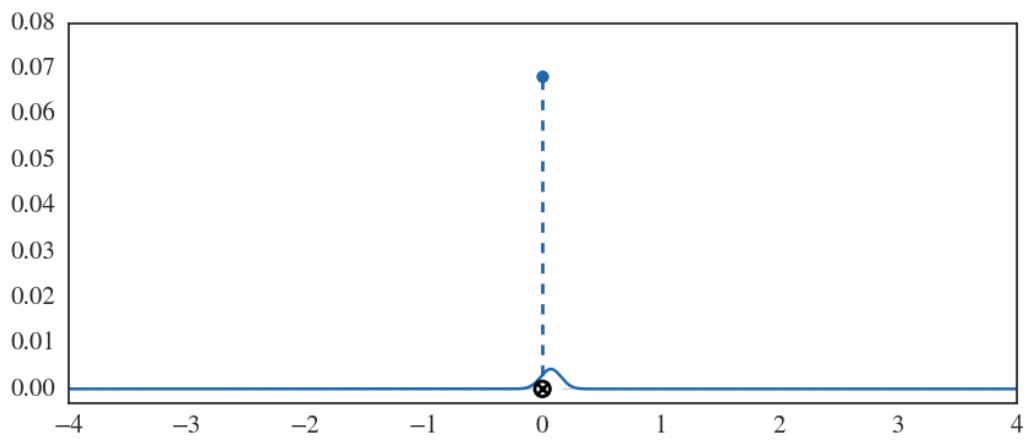

(c) $\alpha=1$

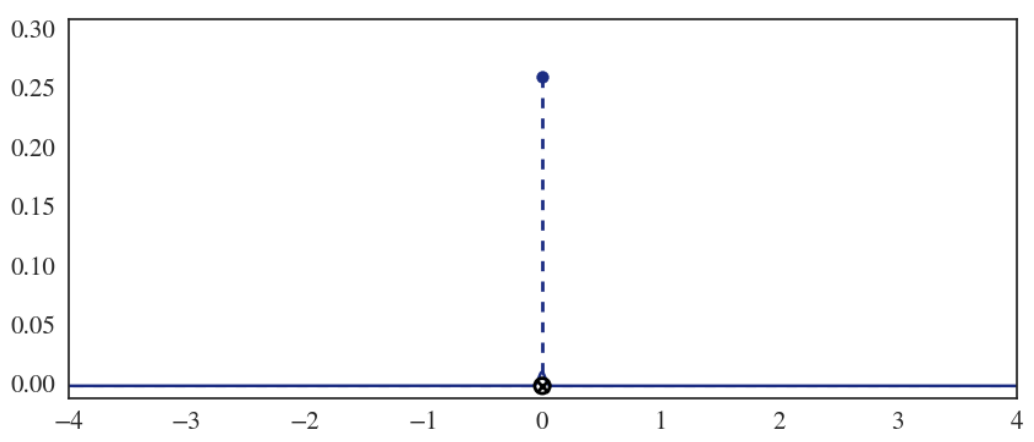

(d) $\alpha=1.5$

Figure 3.5: Evolution of the posterior distribution $\widetilde{P}_{i}\left(x_{i} \mid D^{t}\right)$ for a null component of the signal. The vertical dashed line corresponds to the $\delta\left(x_{i}\right)$ factor in the prior distribution - the dot on its top is the probability $\widetilde{P}_{i}\left(x_{i}=0 \mid D^{t}\right)$. Cross: true value $x_{0, i}$; Circle: signal estimate (posterior mean) $m_{i}^{t}$. 
Notice that the necessary cost of computation for performing (3.38) is $O(N)$ per update. This means that the total computational cost for the recovery when using $t=\alpha N$ measurements is $O\left(N^{2}\right)$, which is comparable to the cost per update of existing fast offline signal recovery algorithms such as the AMP [38, 74, 100]. Also, since at every step all information about previous measurements is discarded except for the parameters $\left\{\left(a_{i}^{t}, h_{i}^{t}\right)\right\}$, the amount of memory necessary to run the online algorithm is of order $O(N)$.

\section{ANALYSIS}

The update equations (3.38) constitute a set of $N$ pairs of stochastic difference equations in which the randomness comes from the variables $\boldsymbol{A}^{t}$ (which are random by design) and $y^{t}$ (in which the randomness comes from possible sources of noise). Figures 3.4 and 3.5 showed a couple of simulation runs for the algorithm, but what can we say about the typical behaviour of such a system? As it turns out, in the limit of $N, t \rightarrow \infty$ (but keeping $\alpha=t / N$ finite), the behaviour is very well defined. In the previous chapter we introduced the replica method to analyze the offline recovery error in this same limit; here we will take a entirely different approach, which nonetheless has many parallels with the results already presented. Observe equations (3.38) and note that since $A_{i}^{t} \sim O\left(N^{-1 / 2}\right)$, the difference with each update for $a_{i}^{t}$ and $h_{i}^{t}$ becomes negligible as $N$ grows. It is this property that allows their reduction to a set of ordinary differential equations by means of:

$$
a_{i}^{t+1}-a_{i}^{t} \simeq \frac{1}{N} \frac{\partial a_{i}}{\partial \alpha} \quad \text { and } \quad h_{i}^{t+1}-h_{i}^{t} \simeq \frac{1}{N} \frac{\partial h_{i}}{\partial \alpha}
$$

In what follows next, differential equations will be derived from the two update equations (3.36), in which the indices $i$ are still preserved on all terms at the R.H.S. of both.

Before going straight to the derivation of these equations, let us introduce an Ansatz for $a_{i}^{t}$ and $h_{i}^{t}$ in the limit $N \rightarrow \infty$. First, notice that in distribution $\widetilde{P}(x)=$ $\exp \left(-a x^{2} / 2+h x\right) \phi(x) / Z$, if one ignores the prior $\phi(x)$ (or, equivalently, make it constant $\phi(x)=c)$ then $a$ acts as the inverse variance and $h$ is strictly related to the expected value of $x$. With $\phi(x)=(1-\rho) \delta(x)+\rho g(x)$ this is clearly not as simple, but nonetheless we propose that the stochastic nature of (3.38) and the statistical uniformity with respect to the site indices $i$ allow us to write

$$
a_{i}^{t} \simeq \hat{Q}(t)
$$


Figure 3.6 shows the evolution of $a_{i}^{t}$ with the number of measurements $t$, demonstrating that they do indeed have all approximately the same value and distribute themselves according to a normal distribution with mean $\hat{Q}$ and variance $\sigma_{a}^{2}$, which goes to zero with $N \rightarrow \infty$.

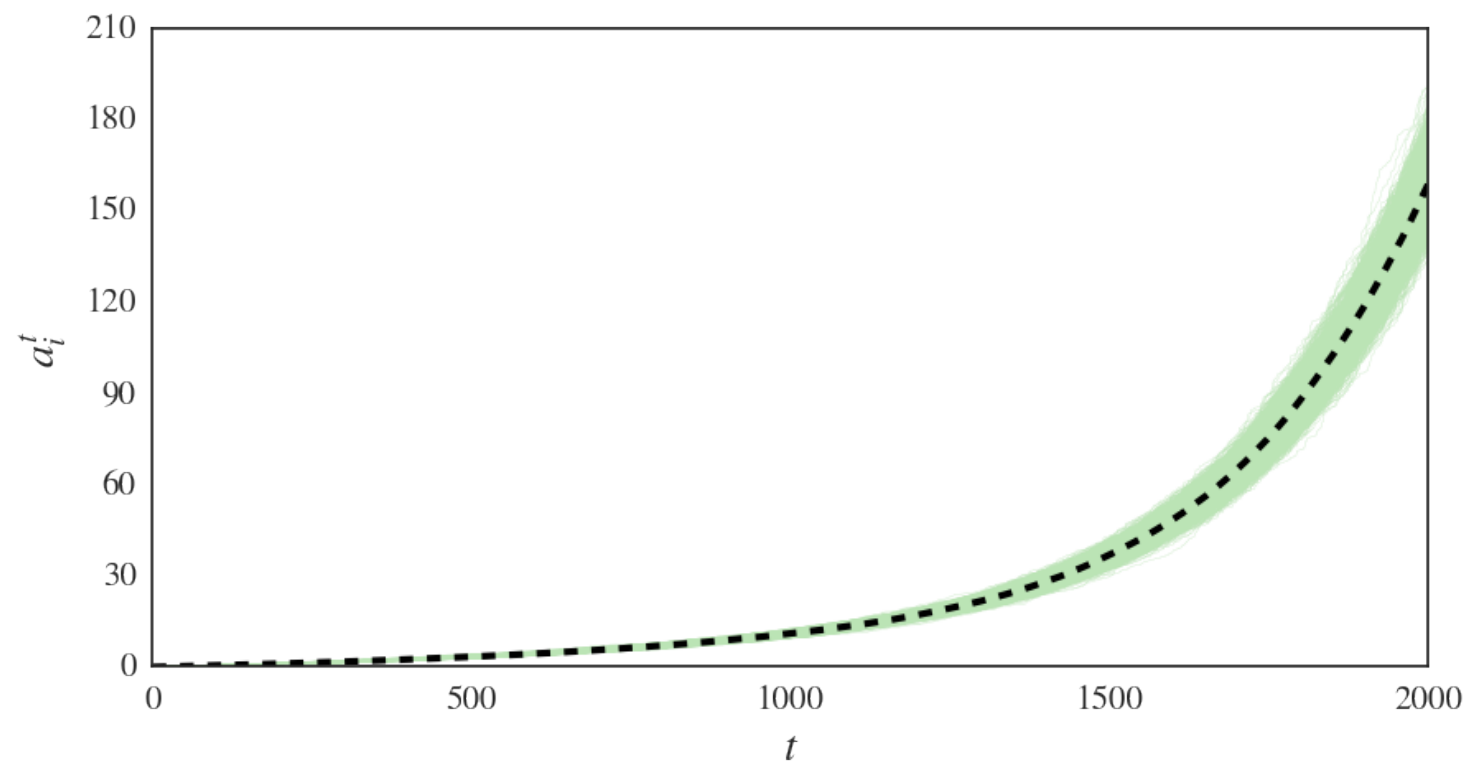

(a) Evolution of $a_{i}^{t}$. Dashed line: mean.

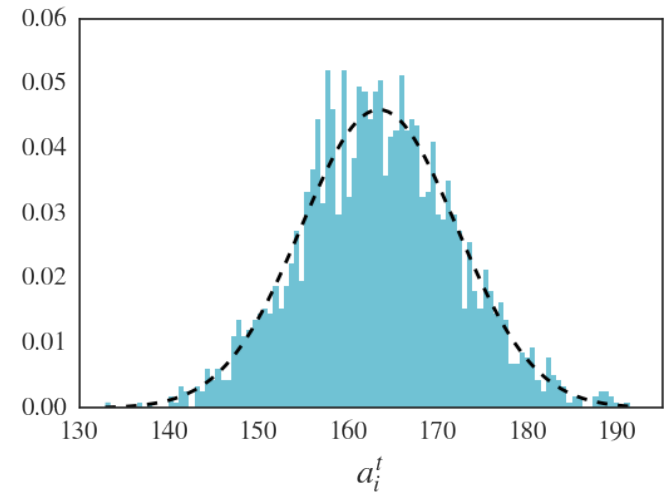

(b) Histogram of $a_{i}^{t}$ at $\alpha=1$.

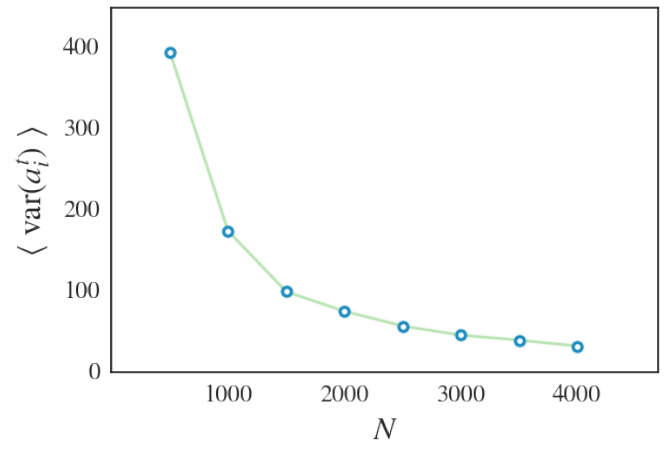

(c) Variance of $a_{i}^{t}$ at $\alpha=1$

Figure 3.6: Evolution of the natural parameters $a_{i}^{t}$ for the noisy standard CS scenario with $N=2000, \sigma_{n}^{2}=10^{-4}$ and a Gauss-Bernoulli prior with $\rho=0.1$ and $\sigma^{2}=1$. Both (a) and (b) are the results of just one simulation and each point on (c) corresponds to the average of 100 simulations. The values of $a_{i}^{t}$ concentrate around a mean value $\hat{Q}(a)$ with variance $\sigma_{a}^{2}(b)$. The value of $\sigma_{a}^{2}$ gets smaller for larger system size $N$, indicating even more concentration around $\hat{Q}$.

As for $h_{i}^{t}$, we expect their values to grow in the direction of $x_{0, i}$, but to maintain a stochastic nature. In Figure 3.7 it is possible to see the evolution of $h_{i}^{t}$ with $t$ and a linear dependence between $h_{i}^{t}$ and $x_{0, i}$. With that in mind, for $z_{i}^{t} \sim \mathcal{N}(0,1)$, we 
propose that

$$
h_{i}^{t} \simeq \hat{m}(t) x_{0, i}+\sqrt{\hat{q}(t)} z_{i}^{t} .
$$

In what follows, the dependency of the macroscopic variables $\hat{Q}, \hat{m}$ and $\hat{q}$ on the time $t$ will be omitted for conciseness.

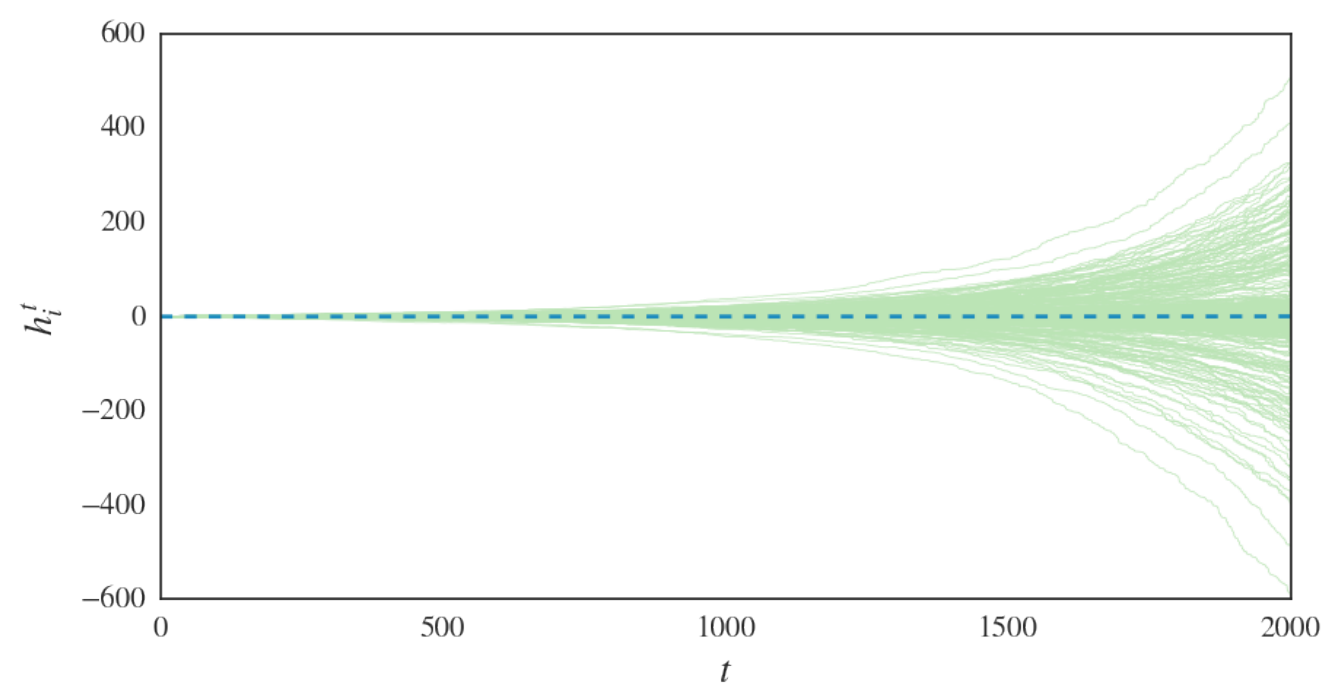

(a) Evolution of $h_{i}^{t}$. Dashed line: mean.

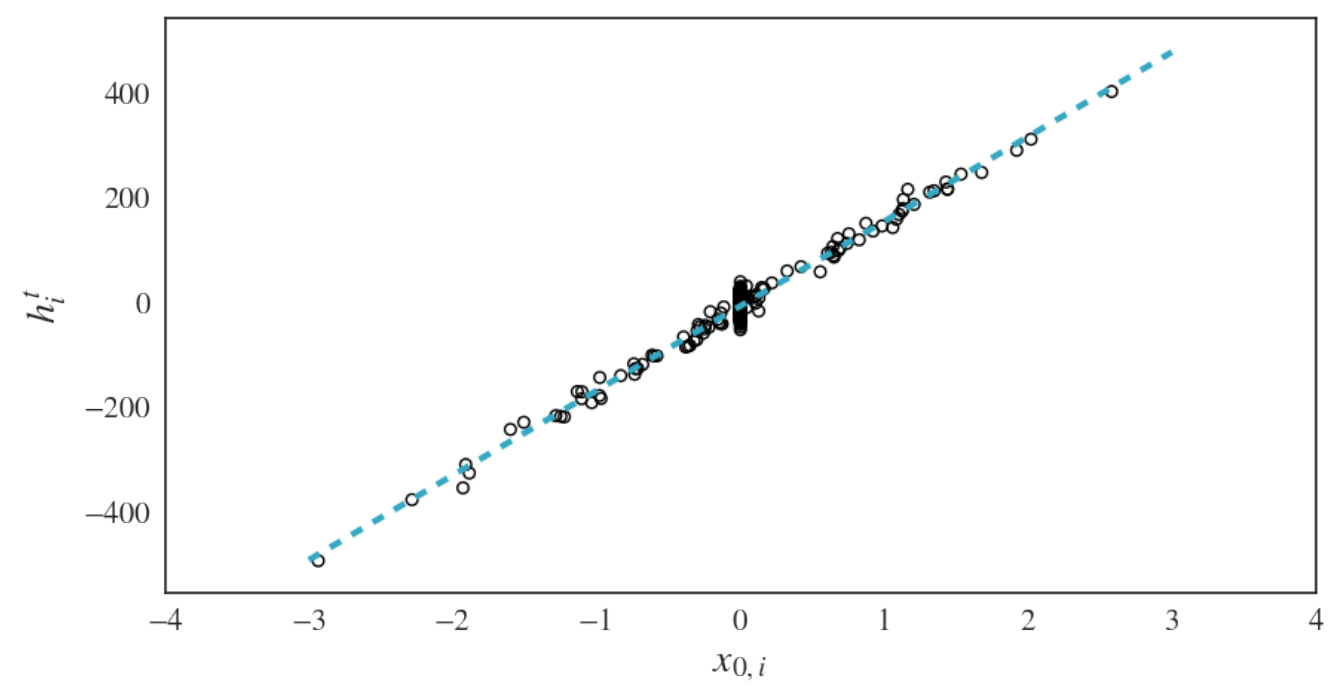

(b) $h_{i}^{t} \times x_{0, i}$ for $\alpha=1$ with linear fit (blue line).

Figure 3.7: Evolution of the natural parameters a $t_{i}^{t}$ for the noisy standard CS scenario with $N=2000, \sigma_{n}^{2}=10^{-4}$ and a Gauss-Bernoulli prior with $\rho=0.1$ and $\sigma^{2}=1$. Both figures correspond to the results of one simulation. The dispersion of $h_{i}^{t}$ at the right end of $(a)$ is not random - in fact, a linear fit of $h_{i}^{t} \times x_{0, i}$ is possible with good precision (b), allowing us to write $h_{i}^{t} \simeq \hat{m}(t) x_{0, i}+\sqrt{\hat{q}(t)} z_{i}^{t}$.

Now consider first the update equation for the parameters $a_{i}^{t}$, repeated here for 
ease of reading:

$$
a_{i}^{t+1}-a_{i}^{t}=-\left(A_{i}^{t+1}\right)^{2} \frac{\partial^{2}}{\partial\left(\Delta_{\backslash i}^{t+1}\right)^{2}}\left(\ln \int \mathcal{D} z P\left(y^{t+1} \mid \Delta_{\backslash i}^{t+1}+\sqrt{\chi_{\backslash i}^{t+1}} z\right)\right) .
$$

If the limit $N \rightarrow \infty$ is taken and both sides of the equality are summed over all indices $i$, we obtain

$$
\frac{1}{N} \frac{\partial}{\partial \alpha} \sum_{i} a_{i}^{t} \simeq-\sum_{i}\left(A_{i}^{t+1}\right)^{2} \frac{\partial^{2}}{\partial\left(\Delta_{\backslash i}^{t+1}\right)^{2}}\left(\ln \int \mathcal{D} z P\left(y^{t+1} \mid \Delta_{\backslash i}^{t+1}+\sqrt{\chi_{\backslash i}^{t+1}} z\right)\right) .
$$

We would like to take the average of both sides with respect to all randomness $\mathbf{A}^{t}$, $y^{t}$ and $\boldsymbol{x}^{0}$ - considering equations (3.36) is what allows such an endeavour to be completed, since in them we take the derivative of the integral with respect to $\Delta_{\backslash i}^{t+1}$, which is not a function of $A_{i}^{t+1}$; if we were considering equations (3.38) instead, the term with $\Delta^{t+1}$ would not be independent of its multiplicative factor and we would not be able to take the average of both terms separately. So taking the expected value of the whole expression results in

$$
\begin{aligned}
\frac{1}{N} \frac{\partial}{\partial \alpha} \sum_{i} a_{i}^{t} & \simeq-\sum_{i}\left\langle\left.\left\langle\left(A_{i}^{t+1}\right)^{2}\right\rangle_{\boldsymbol{A}}\left\langle\frac{\partial^{2}}{\partial\left(\Delta_{\backslash i}^{t+1}\right)^{2}}\left(\ln \int \mathcal{D} z P\left(y^{t+1} \mid \Delta_{\backslash i}^{t+1}+\sqrt{\chi_{\backslash i}^{t+1}} z\right)\right)\right\rangle_{\boldsymbol{A}}\right|_{y, \boldsymbol{x}^{0}}\right. \\
& =-\sum_{i} \frac{1}{N}\left\langle\left.\frac{\partial^{2}}{\partial\left(\Delta_{\backslash i}^{t+1}\right)^{2}}\left(\ln \int \mathcal{D} z P\left(y^{t+1} \mid \Delta_{\backslash i}^{t+1}+\sqrt{\chi_{\backslash i}^{t+1}} z\right)\right)\right|_{\boldsymbol{A}, y, \boldsymbol{x}^{0}}\right. \\
& =-\sum_{i} \frac{1}{N}\left\langle\frac{\partial^{2}}{\partial\left(\Delta^{t+1}\right)^{2}}\left(\ln \int \mathcal{D} z P\left(y^{t+1} \mid \Delta^{t+1}+\sqrt{\chi^{t+1}} z\right)\right)+\left.O\left(A^{t+1}\right)\right|_{\boldsymbol{A}, y, \boldsymbol{x}^{0}}\right. \\
& =-\left\langle\frac{\partial^{2}}{\partial\left(\Delta^{t+1}\right)^{2}}\left(\ln \int \mathcal{D} z P\left(y^{t+1} \mid \Delta^{t+1}+\sqrt{\chi^{t+1}} z\right)\right)\right\rangle_{\boldsymbol{A}, y, \boldsymbol{x}^{0}}
\end{aligned}
$$

From the second to the third line, a Taylor expansion just like in the previous section eliminated the $\backslash i$ indexes, having as collateral effect the appearance of a term proportional to $A_{i}^{t+1}$. In the transition from the third to the fourth line we made use of the fact that $A_{i}^{t+1} \sim \mathcal{N}\left(0, N^{-1}\right)$ to trivially equate this term's expected value to zero. Consideration of the identity (3.40) here transforms the differential equation (3.44) into

$$
\frac{\partial \hat{Q}}{\partial \alpha}=-\left\langle\left.\frac{\partial^{2}}{\partial\left(\Delta^{t+1}\right)^{2}}\left(\ln \int \mathcal{D} z P\left(y^{t+1} \mid \Delta^{t+1}+\sqrt{\chi^{t+1}} z\right)\right)\right|_{\boldsymbol{A}, y, x^{0}} .\right.
$$

A similar procedure can be followed for the update equation for parameters $h_{i}^{t}$ 
in (3.36) (which is also repeated here after exponentiation of both sides):

$$
\left(h_{i}^{t+1}-h_{i}^{t}\right)^{2}=\left(A_{i}^{t+1}\right)^{2}\left(\frac{\partial}{\partial \Delta_{\backslash i}^{t+1}}\left(\ln \int \mathcal{D} z P\left(y^{t+1} \mid \Delta_{\backslash i}^{t+1}+\sqrt{\chi_{\backslash i}^{t+1}} z\right)\right)\right)^{2},
$$

This expression can be worked through as follows. Let us denote changes of $\hat{q}$ and $\hat{m}$ (from expression (3.41)) between measurements $t$ and $t+1$ as $\Delta \hat{q}$ and $\Delta \hat{m}$, respectively, and rewrite the update of $h_{i}^{t}$ as

$$
h_{i}^{t+1}-h_{i}^{t} \simeq \Delta \hat{m} x_{0, i}+\Delta \hat{q} \zeta_{i}^{t}
$$

where the standard normal random variable $\zeta_{i}^{t}$ is independent of $z_{i}^{t}$.

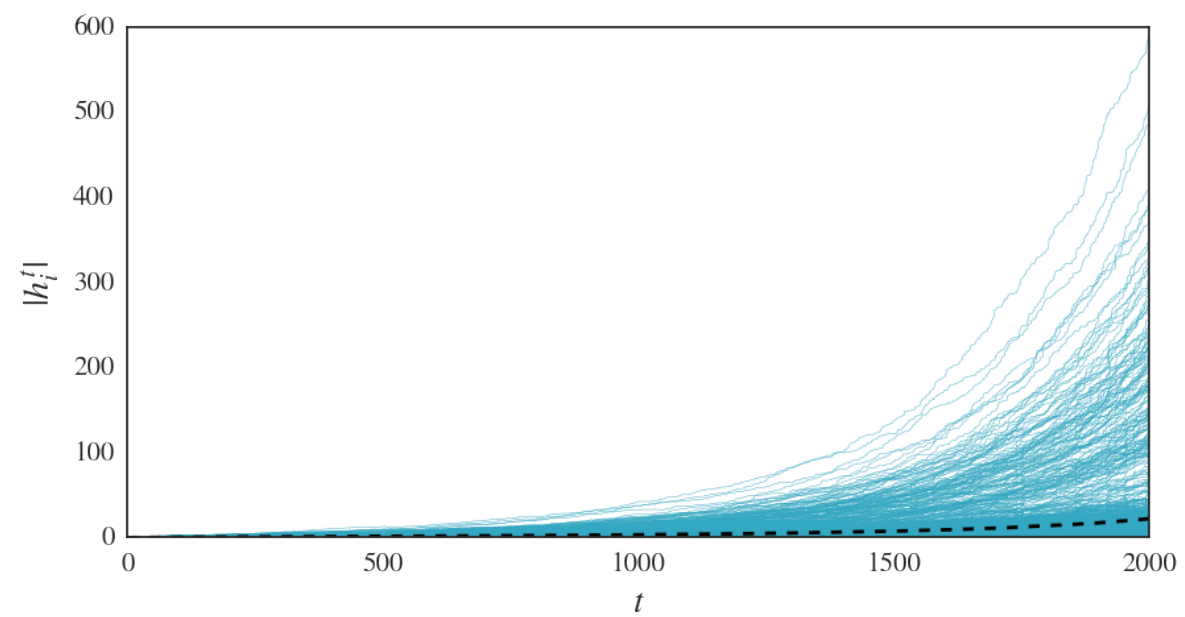

Figure 3.8: Evolution of $\left|h_{i}^{t}\right|$ for one simulation of the noisy standard CS scenario with $N=2000, \sigma_{n}^{2}=10^{-4}$ and a Gauss-Bernoulli prior with $\rho=0.1$ and $\sigma^{2}=1$. Dashed line: mean.

Now, empirically (Figure 3.8) we know that for $t \sim O(N), h_{i}^{t}$ is $O(1)$. This fact implies that the terms $\sqrt{\hat{q}} z_{i}^{t}$ and $\hat{m} x_{0, i}$ - which are constituents of $h_{i}^{t}$ - must also be $O(1)$. Since $\hat{m}$ is the cumulative sum of all $\Delta \hat{m}$, then each of these factors should be $O\left(t^{-1}\right) \sim O\left(N^{-1}\right)$. At the same time, each $\Delta \hat{q}$ is a small increase in the variance of the random process $z_{i}$, so that they must also be $O\left(N^{-1}\right)$. All these considerations imply that if we sum over $\left(h_{i}^{t+1}-h_{i}^{t}\right)^{2}$ and use the law of large numbers to replace sums for their expectations, we will obtain

$$
\begin{aligned}
\sum_{i=1}^{N}\left(h_{i}^{t+1}-h_{i}^{t}\right)^{2} & \simeq \Delta \hat{q} \sum_{i=1}^{N} \zeta_{i}^{2}+2 \sum_{i=1}^{N} \sqrt{\Delta \hat{q}} \Delta \hat{m} \zeta_{i} x_{0, i}+(\Delta \hat{m})^{2} \sum_{i=1}^{N}\left(x_{0, i}\right)^{2} \\
& =\Delta \hat{q}\left\langle\zeta_{i}^{2}\right\rangle+2 \sqrt{\Delta \hat{q}} \Delta \hat{m}\left\langle\zeta_{i} x_{0, i}\right\rangle+(\Delta \hat{m})^{2}\left\langle\left(x_{0, i}\right)^{2}\right\rangle \\
& =\Delta \hat{q}\left\langle\zeta_{i}^{2}\right\rangle+(\Delta \hat{m})^{2}\left\langle\left(x_{0, i}\right)^{2}\right\rangle .
\end{aligned}
$$


Since $(\Delta \hat{m})^{2} \sim O\left(N^{-2}\right)$ is a negligibly small term compared to $\Delta \hat{q} \sum_{i=1}^{N} \zeta_{i}^{2} \simeq N \Delta \hat{q} \simeq$ $d \hat{q} / d \alpha$, which is $O(1)$, we have that

$$
\sum_{i=1}^{N}\left(h_{i}^{t+1}-h_{i}^{t}\right)^{2} \simeq \frac{\partial \hat{q}}{\partial \alpha}
$$

and together with (3.46) this derivative can be calculated to give

$$
\begin{aligned}
\frac{\partial \hat{q}}{\partial \alpha} & \simeq \sum_{i=1}^{N}\left\langle\left\langle\left(A_{i}^{t+1}\right)^{2}\right\rangle_{A}\left\langle\left(\frac{\partial}{\partial \Delta_{\backslash i}^{t+1}}\left(\ln \int \mathcal{D} z P\left(y^{t+1} \mid \Delta_{\backslash i}^{t+1}+\sqrt{\chi_{\backslash i}^{t+1}} z\right)\right)\right)^{2}\right\rangle_{A}\right\rangle_{y, x^{0}} \\
& \simeq \sum_{i=1}^{N}\left\langle\left\langle\left(A_{i}^{t+1}\right)^{2}\right\rangle_{A}\left\langle\left(\frac{\partial}{\partial \Delta^{t+1}}\left(\ln \int \mathcal{D} z P\left(y^{t+1} \mid \Delta^{t+1}+\sqrt{\chi^{t+1}} z\right)\right)\right)^{2}\right\rangle_{A}\right\rangle_{y, x^{0}} \\
& =\left\langle\left(\frac{\partial}{\partial \Delta^{t+1}}\left(\ln \int \mathcal{D} z P\left(y^{t+1} \mid \Delta^{t+1}+\sqrt{\chi^{t+1}} z\right)\right)\right)^{2}\right\rangle_{\boldsymbol{A}, y, x^{0}}
\end{aligned}
$$

To find a similar expression for the last macro-parameter $\hat{m}$, we multiply the $h_{i}^{t}$ update rule in (3.36) for $x_{0, i}$ :

$$
\sum_{i} x_{0, i}\left(h_{i}^{t+1}-h_{i}^{t}\right)=\sum_{i} x_{0, i} A_{i}^{t+1} \frac{\partial}{\partial \Delta_{\backslash i}^{t+1}}\left(\ln \int \mathcal{D} z P\left(y^{t+1} \mid \Delta_{\backslash i}^{t+1}+\sqrt{\chi_{\backslash i}^{t+1}} z\right)\right) .
$$

From (3.41), we have that $\sum_{i}\left\langle h_{i}^{t} x_{0, i}\right\rangle \simeq \sqrt{\hat{q}} \sum_{i}\left\langle z_{i} x_{0, i}\right\rangle+\hat{m} \sum_{i}\left\langle\left(x_{0, i}\right)^{2}\right\rangle=N \hat{m} Q_{0}$, with the definition

$$
Q_{0} \equiv(1 / N) \sum_{i}\left\langle\left(x_{0, i}\right)^{2}\right\rangle .
$$

Therefore, taking the expected value of both sides of (3.51) results in

$$
\begin{aligned}
\frac{\partial \hat{m}}{\partial \alpha} & \simeq \frac{1}{Q_{0}}\left\langle\left.\sum_{i} x_{0, i} A_{i}^{t+1} \frac{\partial}{\partial \Delta_{\backslash i}^{t+1}}\left(\ln \int \mathcal{D} z P\left(y^{t+1} \mid \Delta_{\backslash i}^{t+1}+\sqrt{\chi_{\backslash i}^{t+1}} z\right)\right)\right|_{\boldsymbol{A}, y, x^{0}}\right. \\
& =\frac{1}{Q_{0}} \int d y\left\langle\sum_{i} x_{0, i} A_{i}^{t+1} P\left(y \mid \Delta_{0}^{t+1}\right) \frac{\partial}{\partial \Delta_{\backslash i}^{t+1}}\left(\ln \int \mathcal{D} z P\left(y^{t+1} \mid \Delta_{\backslash i}^{t+1}+\sqrt{\chi_{\backslash i}^{t+1}} z\right)\right)\right\rangle_{\boldsymbol{A}, x^{0}}
\end{aligned}
$$

where the expected value on $y \sim P(y \mid u)$ was made explicit. If we define, just like we had defined the $\Delta^{t}$ and $\Delta_{\backslash i}^{t}$ factors above, the projections

$$
\Delta_{0, \backslash i}^{t+1} \equiv \sum_{j \neq i} A_{j}^{t+1} x_{0, j} \quad \text { and } \quad \Delta_{0}^{t+1} \equiv \sum_{j} A_{j}^{t+1} x_{0, j}
$$


we can make use of another Taylor expansion to expand $P\left(y \mid \Delta_{0}^{t+1}\right)$ :

$$
P\left(y \mid \Delta_{0}^{t+1}\right) \simeq P\left(y \mid \Delta_{0, \backslash i}^{t+1}\right)+\left(x_{0, i} A_{i}^{t+1}\right) \frac{\partial}{\partial \Delta_{0, \backslash i}^{t+1}} P\left(y \mid \Delta_{0, \backslash i}^{t+1}\right)+O\left(\left(A_{i}^{t+1}\right)^{2}\right) .
$$

Therefore,

$$
\begin{aligned}
\frac{\partial \hat{m}}{\partial \alpha}=\frac{1}{Q_{0}} \int d y\langle & \sum_{i} x_{0, i} A_{i}^{t+1}\left[P\left(y \mid \Delta_{0, i}^{t+1}\right)+\left(x_{0, i} A_{i}^{t+1}\right) \frac{\partial}{\partial \Delta_{0, i}^{t+1}} P\left(y \mid \Delta_{0, i i}^{t+1}\right)\right] \times \\
& \times\left.\frac{\partial}{\partial \Delta_{\backslash i}^{t+1}}\left(\ln \int \mathcal{D} z P\left(y^{t+1} \mid \Delta_{\backslash i}^{t+1}+\sqrt{\chi_{\backslash i}^{t+1}} z\right)\right)\right|_{A, x^{0}} \\
=\frac{1}{Q_{0}} \int d y \sum_{i} & {\left[\left\langle x_{0, i} A_{i}^{t+1}\right\rangle \times\right.} \\
& \times\left\langle P\left(y \mid \Delta_{0, i}^{t+1}\right) \frac{\partial}{\partial \Delta_{\backslash i}^{t+1}}\left(\ln \int \mathcal{D} z P\left(y^{t+1} \mid \Delta_{\backslash i}^{t+1}+\sqrt{\chi_{\backslash i}^{t+1}} z\right)\right)\right\rangle+ \\
& \left\langle\left(x_{0, i} A_{i}^{t+1}\right)^{2}\right\rangle \times \\
& \times\left\langle\frac{\partial}{\partial \Delta_{0, i}^{t+1}} P\left(y \mid \Delta_{0, \backslash i}^{t+1}\right) \frac{\partial}{\partial \Delta_{\backslash i}^{t+1}}\left(\ln \int \mathcal{D} z P\left(y^{t+1} \mid \Delta_{\backslash i}^{t+1}+\sqrt{\chi_{\backslash i}^{t+1}} z\right)\right)\right\rangle .
\end{aligned}
$$

As $\left\langle A_{i}^{t+1}\right\rangle=0$ and $\sum_{i}\left(x_{0, i}\right)^{2}\left\langle\left(A_{i}^{t+1}\right)^{2}\right\rangle_{A^{t+1}}=(1 / N) \sum_{i}\left(x_{0, i}\right)^{2}=Q_{0}$,

$$
\begin{aligned}
\frac{\partial \hat{m}}{\partial \alpha} & =\int d y\left\langle\left.\frac{\partial}{\partial \Delta_{0, \backslash i}^{t+1}} P\left(y \mid \Delta_{0, \backslash i}^{t+1}\right) \frac{\partial}{\partial \Delta_{\backslash i}^{t+1}}\left(\ln \int \mathcal{D} z P\left(y^{t+1} \mid \Delta_{\backslash i}^{t+1}+\sqrt{\chi_{\backslash i}^{t+1}} z\right)\right)\right|_{\boldsymbol{A}, \boldsymbol{x}^{0}}\right. \\
& \simeq \int d y\left\langle\left.\frac{\partial}{\partial \Delta_{0}^{t+1}} P\left(y \mid \Delta_{0}^{t+1}\right) \frac{\partial}{\partial \Delta^{t+1}}\left(\ln \int \mathcal{D} z P\left(y^{t+1} \mid \Delta^{t+1}+\sqrt{\chi^{t+1}} z\right)\right)\right|_{\boldsymbol{A}, \boldsymbol{x}^{0}}\right.
\end{aligned}
$$

Equations (3.45), (3.50) and (3.57) are all functions of $\Delta_{0}^{t}, \Delta^{t}$ and $\chi^{t}$. They can assume simpler forms with the introduction of these variable's first and second moments. As sums of normal random variables, $\Delta_{0}^{t}$ and $\Delta^{t}$ also follow a multivariate normal distribution with mean

$$
\begin{aligned}
& \left\langle\Delta_{0}^{t}\right\rangle=\sum_{i=1}^{N}\left\langle A_{i}^{t}\right\rangle x_{0, i}=0 \\
& \left\langle\Delta^{t}\right\rangle=\sum_{i=1}^{N}\left\langle A_{i}^{t}\right\rangle m_{i}=0
\end{aligned}
$$


and covariance

$$
\begin{aligned}
\left\langle\left(\Delta^{t}\right)^{2}\right\rangle=\sum_{i=1}^{N} \sum_{j=1}^{N}\left\langle A_{i}^{t} A_{j}^{t}\right\rangle m_{i} m_{j} & =\sum_{i=1}^{N} \sum_{j=1}^{N} \frac{1}{N} \delta_{i j} m_{i} m_{j}=\frac{1}{N} \sum_{i=1}^{N} m_{i}^{2} \\
\left\langle\left(\Delta_{0}^{t}\right)^{2}\right\rangle & =\frac{1}{N} \sum_{i=1}^{N}\left(x_{0, i}\right)^{2} \\
\left\langle\Delta_{0}^{t} \Delta^{t}\right\rangle & =\frac{1}{N} \sum_{i=1}^{N} x_{0, i} m_{i}
\end{aligned}
$$

where $\langle\cdots\rangle$ denotes the average with respect to the measurement vectors $\boldsymbol{A}^{t}$. Let us name the covariates $\left\langle\left(\Delta^{t}\right)^{2}\right\rangle \equiv q,\left\langle\left(\Delta_{0}^{t}\right)^{2}\right\rangle \equiv Q_{0}$ and $\left\langle\Delta_{0}^{t} \Delta^{t}\right\rangle \equiv m$. Then

$$
\left\langle\chi^{t}\right\rangle=\sum_{i}^{N}\left\langle\left(A_{i}^{t}\right)^{2}\right\rangle v_{i}=\underbrace{\sum_{i}^{N}\left\langle\left(A_{i}^{t}\right)^{2}\right\rangle\left\langle x_{i}^{2}\right\rangle}_{\equiv Q}-\sum_{i}^{N}\left\langle\left(A_{i}^{t}\right)^{2}\right\rangle m_{i}=Q-q
$$

and its variance is proportional to $N^{-2}$. The results above mean that we can write $\Delta_{t}$ and $\Delta_{t}^{0}$ as sums of independent standard Gaussians $u$ and $v$ :

$$
\left\{\begin{aligned}
\Delta^{t} & \equiv \sqrt{q} v \\
\Delta_{0}^{t} & \equiv \sqrt{Q_{0}-\frac{m^{2}}{q}} u+\frac{m}{\sqrt{q}} v
\end{aligned}\right.
$$

At the same time, up to order $N^{-1}$,

$$
\chi^{t} \equiv Q-q
$$

Expliciting

$$
\left\langle x_{i}\right\rangle=\frac{\partial}{\partial(\sqrt{\hat{q}} z)} \ln Z\left(\hat{Q}, \sqrt{\hat{q}} z+\hat{m} x_{0, i}\right),
$$

where $Z(a, h)=\int d x \phi(x) e^{-a x^{2} / 2+h x}$, and introducing the averages on $y$ and $\boldsymbol{x}^{0}$ yields the analytic expressions

$$
\left\{\begin{array}{l}
Q_{0}=\int d x \phi(x) x^{2} \\
m=\int d x^{0} \phi\left(x^{0}\right) \mathcal{D} z x^{0}\langle x\rangle, \\
q=\int d x^{0} \phi\left(x^{0}\right) \mathcal{D} z\langle x\rangle^{2}, \\
Q=q+\int d x^{0} \phi\left(x^{0}\right) \mathcal{D} z \frac{\partial\langle x\rangle}{\partial(\sqrt{\hat{q}} z)} .
\end{array}\right.
$$


With these, equations (3.45), (3.50) and (3.57) can be written, respectively,

$$
\left\{\begin{aligned}
\frac{\partial \hat{Q}}{\partial \alpha} \simeq-\int d y \int \mathcal{D} u \int \mathcal{D} v\left[P\left(y \mid \sqrt{Q_{0}-\frac{m^{2}}{q}} u+\frac{m}{\sqrt{q}} v\right) \times\right. \\
\left.\times \frac{\partial^{2}}{\partial(\sqrt{q} v)^{2}}\left(\ln \int \mathcal{D} z P(y \mid \sqrt{q} v+\sqrt{Q-q} z)\right)\right] \\
\frac{\partial \hat{q}}{\partial \alpha} \simeq \int d y \int \mathcal{D} u \int \mathcal{D} v\left[P\left(y \mid \sqrt{Q_{0}-\frac{m^{2}}{q}} u+\frac{m}{\sqrt{q}} v\right) \times\right. \\
\left.\times\left(\frac{\partial}{\partial(\sqrt{q} v)}\left(\ln \int \mathcal{D} z P(y \mid \sqrt{q} v+\sqrt{Q-q} z)\right)\right)^{2}\right] \\
\frac{\partial \hat{m}}{\partial \alpha} \simeq \int d y \int \mathcal{D} u \int \mathcal{D} v\left[\frac{\partial}{\partial(m v / \sqrt{q})} P\left(y \mid \sqrt{Q_{0}-\frac{m^{2}}{q}} u+\frac{m}{\sqrt{q}} v\right) \times\right. \\
\left.\times \frac{\partial}{\partial(\sqrt{q} v)}\left(\ln \int \mathcal{D} z P(y \mid \sqrt{q} v+\sqrt{Q-q} z)\right)\right] .
\end{aligned}\right.
$$

They are a set of partial differential equations

$$
\frac{\partial \hat{Q}}{\partial \alpha}=f_{1}\left(Q_{0}, Q, q, m\right), \frac{\partial \hat{q}}{\partial \alpha}=f_{2}\left(Q_{0}, Q, q, m\right), \frac{\partial \hat{m}}{\partial \alpha}=f_{3}\left(Q_{0}, Q, q, m\right) .
$$

that fully describe the reconstruction behaviour of the online algorithm in the limit $N \rightarrow \infty$. With them, the performance of the algorithm in the large $N$ limit can then be calculated through the following scheme which summarizes the results in the last paragraphs:

Scheme for calculation of the macroscopic behaviour of the Bayesian Online Compressed Sensing Algorithm for $N \rightarrow \infty$

1: Given $\hat{Q}, \hat{q}$ and $\hat{m}$;

2: Calculate $Q(\hat{Q}, \hat{q}, \hat{m}), q(\hat{Q}, \hat{q}, \hat{m})$ and $m(\hat{Q}, \hat{q}, \hat{m})$;

3: Update $\hat{Q}, \hat{q}$ and $\hat{m}$ through eqs. (3.67);

4: The normalized reconstruction error is given by the normalized mse $=\left(Q_{0}-2 m+\right.$ q)/Q $Q_{0}$

Three issues are of note here. First off, note that the macroscopic variables $Q_{0}, Q, q$ and $m$ defined in this section have the same meaning as the overlap variables in the replica-symmetric calculation of the Bayesian offline signal recovery scheme; thus, the naming of the variables was far from accidental. Second, replacing the triad $(d \hat{Q} / d \alpha, d \hat{q} / d \alpha, d \hat{m} / d \alpha)$ with $(\hat{Q} / \alpha, \hat{q} / \alpha, \hat{m} / \alpha)$ in (3.69) yields the exact equation of state for the Bayesian offline signal recovery (obtained through derivation of the free 
energy (2.16)). The mathematical implication is that whatever existing performance limitation of the online method (as compared to the offline method) arrives from the differences in the macroscopic descriptions - i.e., the use of differential instead of algebraic equations. Third, the equations of state (3.69) allow a solution with $\hat{Q}=\hat{q}=\hat{m}, Q=Q_{0}$, and $q=m$. This corresponds to the Nishimori line, when the signal distribution $\phi_{0}(x)$ is perfectly known. Focusing on the solution of this type simplifies (3.69) and (3.67) to

$$
\left\{\begin{aligned}
q=\int d x^{0} \phi\left(x^{0}\right) \mathcal{D} z\left(\partial / \partial(\sqrt{\hat{q}} z) \ln Z\left(\hat{q}, \sqrt{\hat{q}} z+\hat{q} x^{0}\right)\right)^{2} \\
\frac{d \hat{q}}{d \alpha}=\int d y \int \mathcal{D} v\left[\int \mathcal{D} u P\left(y \mid \sqrt{q} v+\sqrt{Q_{0}-q} u\right) \times\right. \\
\left.\times\left(\frac{\partial}{\partial \sqrt{q} v} \ln \int \mathcal{D} u P\left(y \mid \sqrt{q} v+\sqrt{Q_{0}-q} u\right)\right)^{2}\right]
\end{aligned}\right.
$$

In order to test the algorithm and the macroscopic description above, we simulated two representative scenarios of Compressed Sensing. Since real life applications always suffer from a certain amount of measurement corruption, for practical relevance of the framework we considered situations where each measurement was degraded by Gaussian noise of zero mean and variance $\sigma_{n}^{2}$ for both cases. The first scenario is the standard CS, which has been mentioned many times here before and is characterized by $P(y \mid u)=\left(2 \pi \sigma_{n}^{2}\right)^{-1 / 2} \exp \left(-(y-u)^{2} /\left(2 \sigma_{n}^{2}\right)\right)$. The other is the 1-bit CS (Appendix B), which in the presence of noise is modeled by $P(y \mid u)=$ $\int \mathcal{D} z \Theta\left(y u+\sigma_{n} z\right)$. Here, $y \in\{+1,-1\}$, and $\Theta(x)=1$ for $x \geq 0$ and 0 otherwise. In all examples the Gauss-Bernoulli prior $\phi(x)=(1-\rho) \delta(x)+\rho\left(2 \pi \sigma^{2}\right)^{-1 / 2} \exp \left(-x^{2} /\left(2 \sigma^{2}\right)\right)$ was considered, implying in $Q_{0}=\rho \sigma^{2}$.

Figures 3.9 and 3.10 compare the normalized reconstruction error mse from the experimental results obtained with (3.38) and the theoretical predictions of this section. For standard CS (Figure 3.9), where the main interest still lies in making less measurements than the dimension of the signal, our analysis was constrained to $\alpha \leq 1$. But in the 1-bit CS case, measurements are considered cheap whilst data transmission and/or storage are costly [14], justifying the analysis of the performance of the algorithm for $\alpha>1$. The experimental results represent averages over 1000 samples, while the theoretical predictions were evaluated by solving the system of equations (3.70) with the use of the Runge-Kutta method. The empirical data extrapolated to $N \rightarrow \infty$ (Figure 3.11) exhibits excellent agreement with the theoretical predictions.

Fig. 3.12 compares the reconstruction error mse from the theoretical predictions 

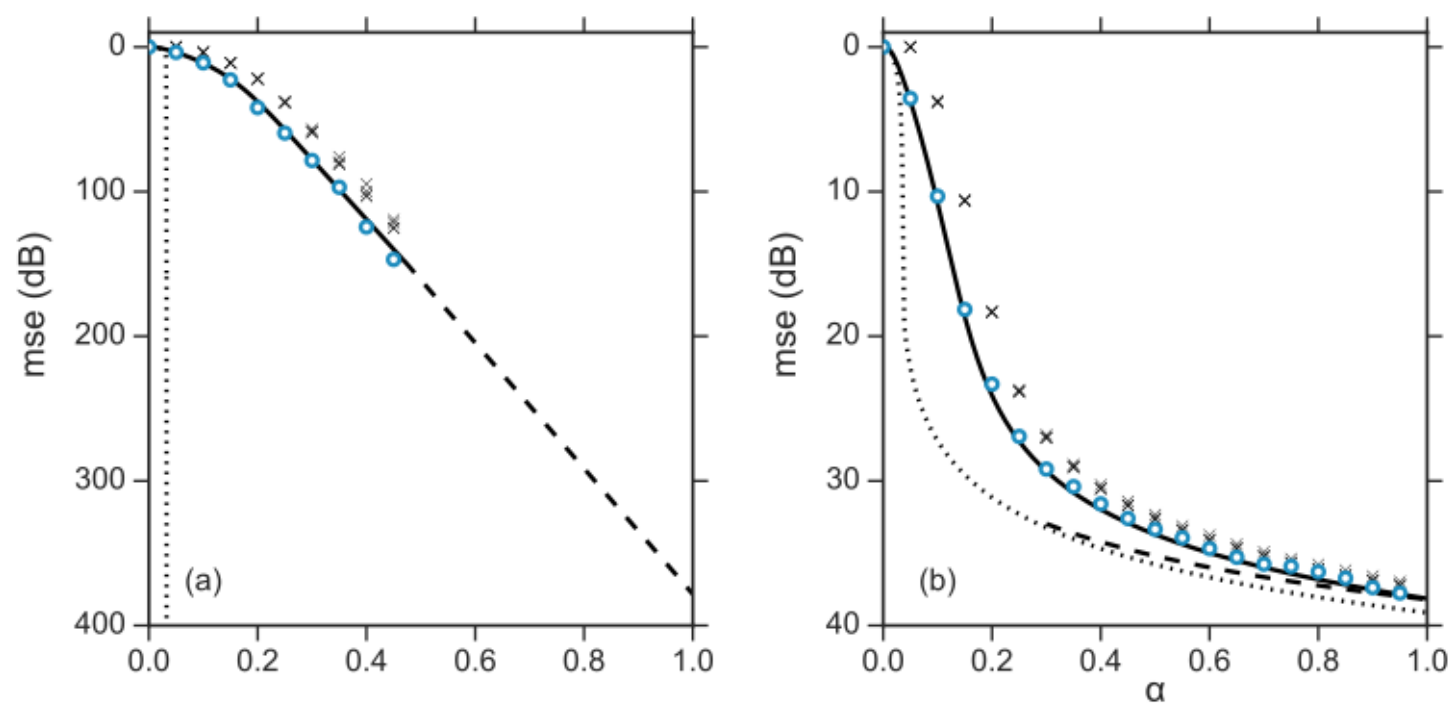

Figure 3.9: Standard $\boldsymbol{C S}$ scenario with $\rho=0.01$ and $\sigma^{2}=1$. Comparison between the mse normalized by $Q_{0}$ from experimental results and theoretical predictions for the (a) noiseless $\left(\sigma_{n}^{2}=0\right)$ and (b) noisy $\left(\sigma_{n}^{2}=10^{-4}\right)$ cases. The crosses (in descending order) correspond to the average of 1000 simulations with $N=2000,3000,4000$, and 5000 and the blue circles are the extrapolations of the data to $N \rightarrow \infty$ by quadratic fitting. The lines represent the theoretical performances of the online (continuous) and offline (dotted) reconstructions, and the asymptotic dynamics of (3.70) (dashed). In (a), the macroscopic dynamics is truncated because of numerical limitations of the computational environment utilized. Also in this case, offline reconstruction gives mse $=0$ for $\alpha>\alpha_{c}(\rho)$.

(3.70) of the online and offline methods for the standard CS case. It becomes clear that the drawback of the utilization of the online method is only a small loss of performance which becomes even smaller with larger noise. On the other hand, considerable computational effort can be saved by its use.

\section{Asymptotic AnALYsis}

In the presence of Gaussian measurement noise, the differentiability of $P(y \mid u)$ with respect to $u$ guarantees that in the asymptotic limit defined by $\hat{q} \rightarrow \infty$ and $q \rightarrow Q_{0}$, the right-hand side in the second equation of (3.70) becomes a constant. This implies that $\hat{q} \simeq I \alpha$ asymptotically holds in the online reconstruction, yielding an universal expression for the asymptotic normalized error: $\mathrm{mse} \simeq \rho / \hat{q}=\rho /(I \alpha)$, where

$$
I=\int d y \int \mathcal{D} v P\left(y \mid \sqrt{Q_{0}} v\right)\left(\frac{\partial}{\partial \sqrt{Q_{0}} v} \ln P\left(y \mid \sqrt{Q_{0}} v\right)\right)^{2} .
$$

Remarkably, this is the same expression obtained for the offline reconstruction (e.g., [137]). This shows the potential utility of the Bayesian Online CS algorithm and 

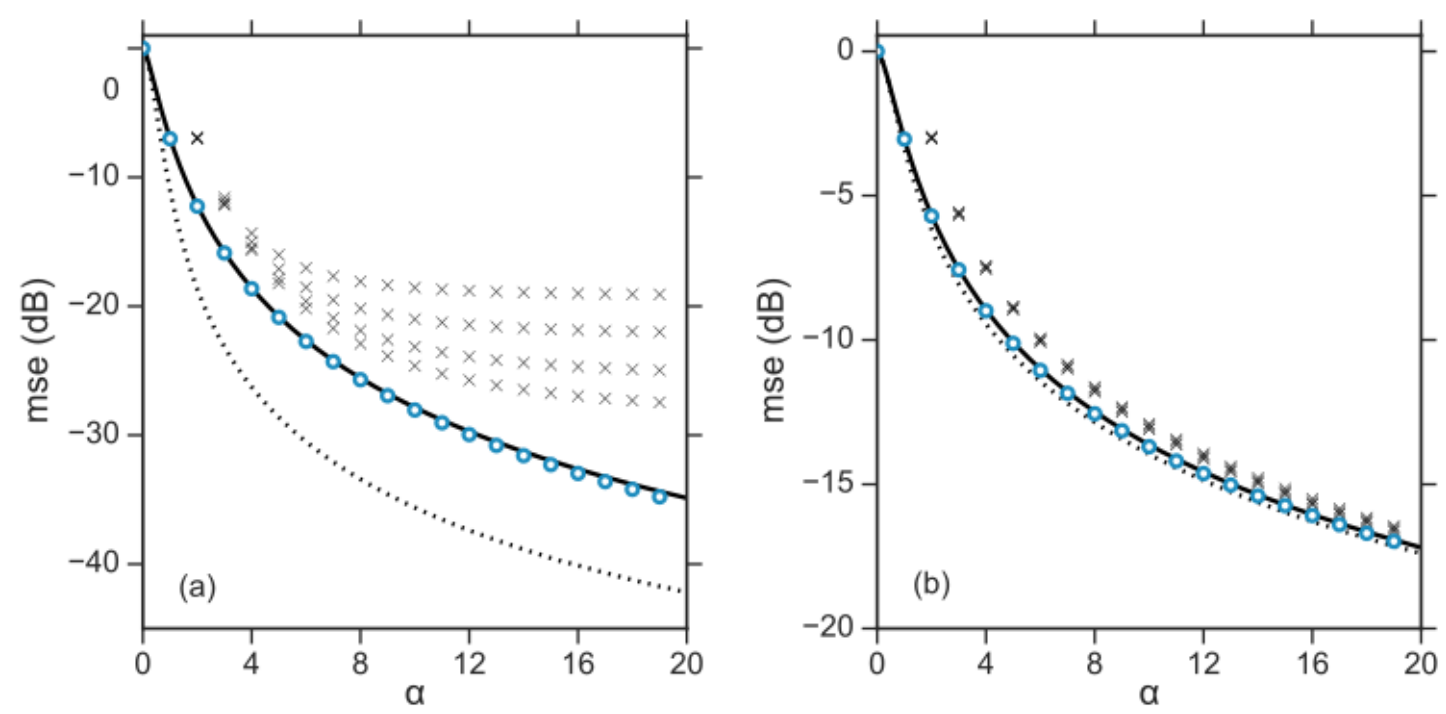

Figure 3.10: 1-bit $\boldsymbol{C S}$ scenario with $\rho=0.1$ and $\sigma^{2}=1$. Comparison between the mse normalized by $Q_{0}$ from experimental results and theoretical predictions for the (a) noiseless $\left(\sigma_{n}^{2}=0\right)$ and (b) noisy $\left(\sigma_{n}^{2}=10^{-1}\right)$ cases. The crosses (in descending order) correspond to the average of 1000 simulations with $N=500,1000,2000$, and 4000 and the blue circles are the extrapolations of the data to $N \rightarrow \infty$ by quadratic fitting. The lines represent the theoretical performances of the online (continuous) and offline (dotted) reconstructions.

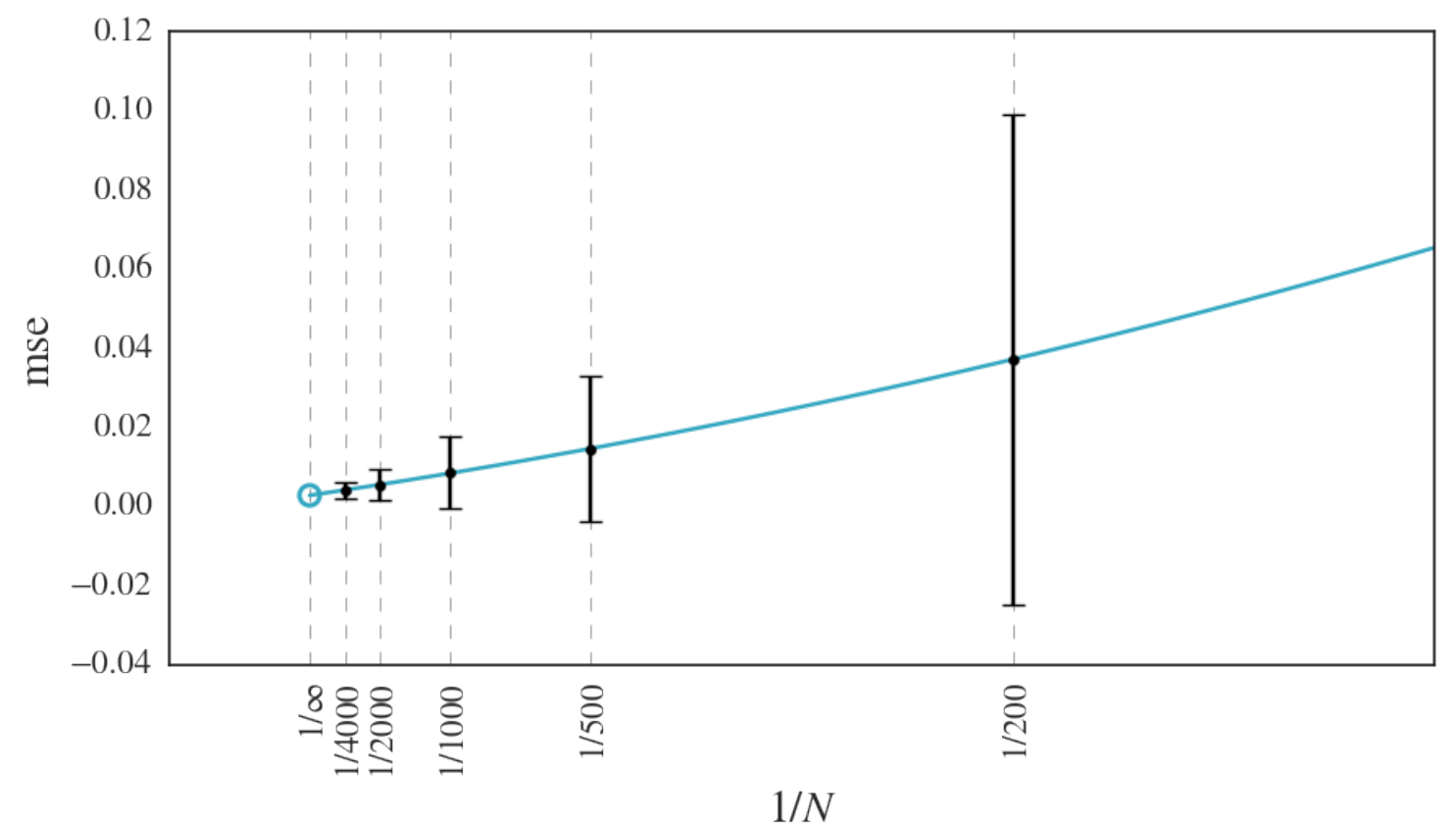

Figure 3.11: Example of a quadratic fit to eliminate the finite size effect present in the simulations. 1-bit $\boldsymbol{C S}$ scenario with $\alpha=0.8, \rho=0.1$ and $\sigma^{2}=1$.

indicates that a performance similar to that of the offline method can be asymptotically achieved with a significant reduction in computational costs. For standard CS with very sparse signals where $\rho \ll 1$ this asymptotic regime can be found already 


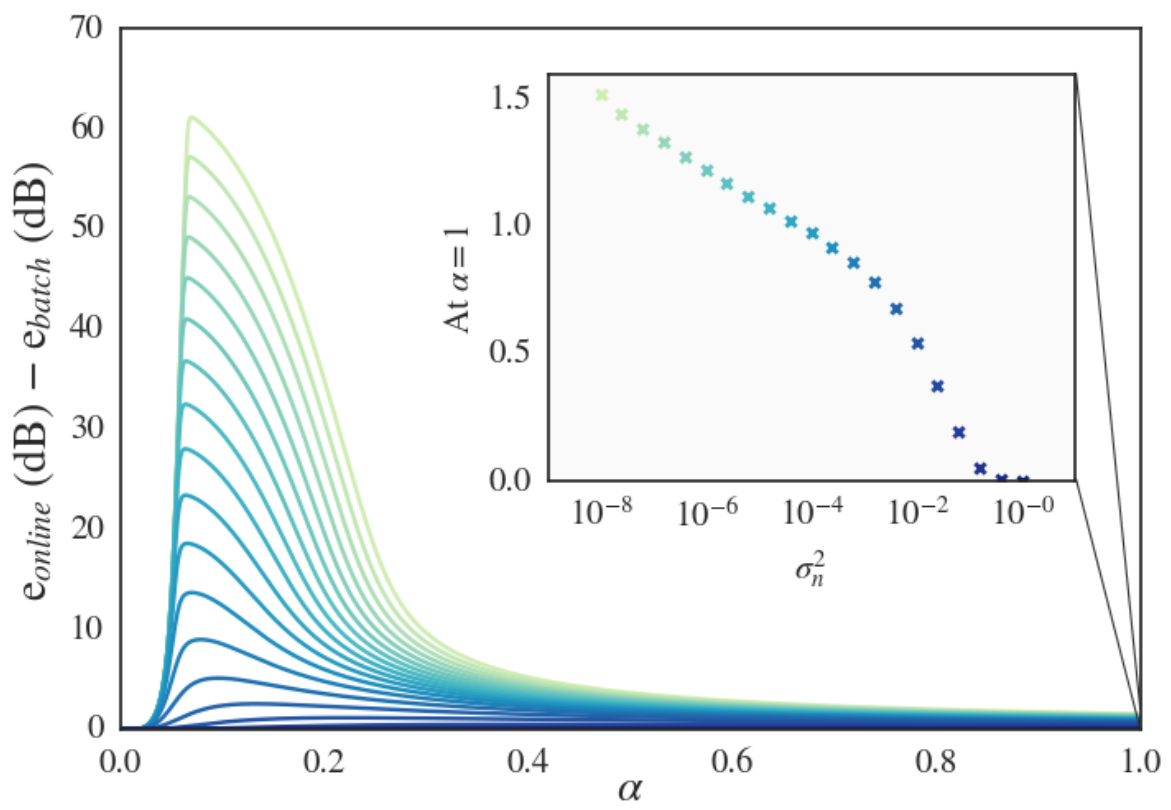

Figure 3.12: Theoretical differences between the normalized mse from the predictions of the online and offline reconstruction in the Standard $\boldsymbol{C S}$ scenario with $\rho=0.01$ and $\sigma^{2}=1$. Lines correspond to the noise variance $\sigma_{n}^{2}$ equal to $10^{-8}$ (dashed), $10^{-5}$ (dot-dashed) and $10^{-2}$ (dotted). Inset: The differences calculated at $\alpha=1$.

for $\alpha<1$ (Figure (3.9)(b)).

In the noiseless scenario the situation is different - there is a fundamental gap in the asymptotically achievable performance limit. For noiseless standard CS, the offline reconstruction achieves mse $=0$ whenever $\alpha$ is greater than a certain critical ratio $0<\alpha_{\mathrm{c}}(\rho)<1$ (Chapter 2). On the other hand, analysis of (3.70) indicates that $\mathrm{mse} \simeq O(\exp (-\alpha / \rho))$ holds for the normalized error when $\alpha / \rho$ is large (Figure $(3.9)(a))$, which confirms our intuition that even in this scenario perfect recovery is, unfortunately, impossible with the Bayesian Online CS algorithm. However, this result may still promote the use of the online algorithm for very sparse signals with $0<\rho \ll \alpha$ where $\exp (-\alpha / \rho)$ becomes negligible. For noiseless 1-bit CS, it is possible to show that mse $\simeq Q_{0}\left(\frac{\rho}{2 K \alpha}\right)^{2}$ is asymptotically achieved by the offline method, where $K=0.3603 \ldots$ [137]. For the online scenario, solution of (3.70) yields the asymptotic form mse $\simeq Q_{0}\left(\frac{\rho}{K \alpha}\right)^{2}$ for $\alpha \gg 1$. This means the same fundamental gap is found here and indicates that online recovery can save computation and memory costs considerably while sacrificing mse by only a factor 4 asymptotically.

The dependence on $\alpha$ on the asymptotic results demonstrates that the error does never vanish in the online reconstruction scenario. This comes in contrast to the offline scenario, where the stabilization of the signal estimate can provide heuristic rules to determine the necessity (or not) of performing more measurements in order 
to achieve perfect reconstruction [80]. Such a method is not possible here, but the possibility remains open for inversion of these same asymptotic results in order to calculate a threshold $\alpha_{\mathrm{thr}}$ for which an acceptable error is met. 



\section{CHAPTER 4}

\section{MismatCHED PRIORS}

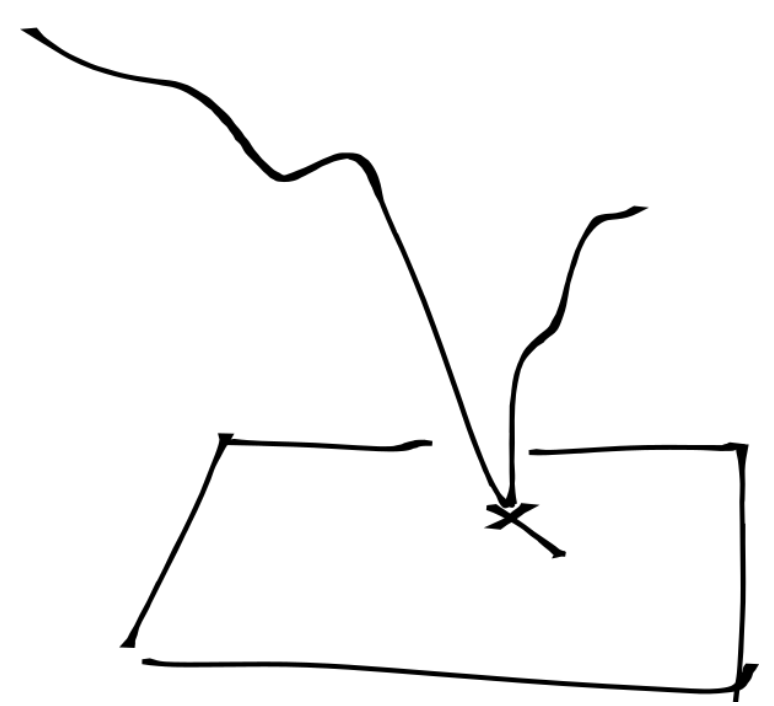

Rossi, Paulo V. and Vicente, R.

L1-Minimization Algorithm for Bayesian Online Compressed Sensing Entropy 19 (2017): 667 


\subsection{INTRODUCTION}

A limitation of the Bayesian Online Compressed Sensing algorithm introduced in the previous chapter is the simple fact that in a real-world scenario one does not know the exact parameters of the generating distribution, and possibly not even some of its major characteristics except for the fact that the generated signals are somewhat sparse. Even in the most controlled scenarios, exactitude should not be expected and an ideal algorithm should be robust to at least partially incorrect assumptions. As can be seen in the examples in Figure 4.1, if one introduces a prior $\phi(x)$ in the projected distribution $\widetilde{P}\left(\boldsymbol{x} \mid D^{t}\right)$ (equation (3.23)) which is different from the actual function $\phi_{0}(x)$ that generated the signal, the reconstruction accuracy drops significantly. For the same number of measurements, the signal's estimate can be considerably worse (Figure 4.2). Therefore there is a real necessity to extend the formalism above to these more realistic situations.

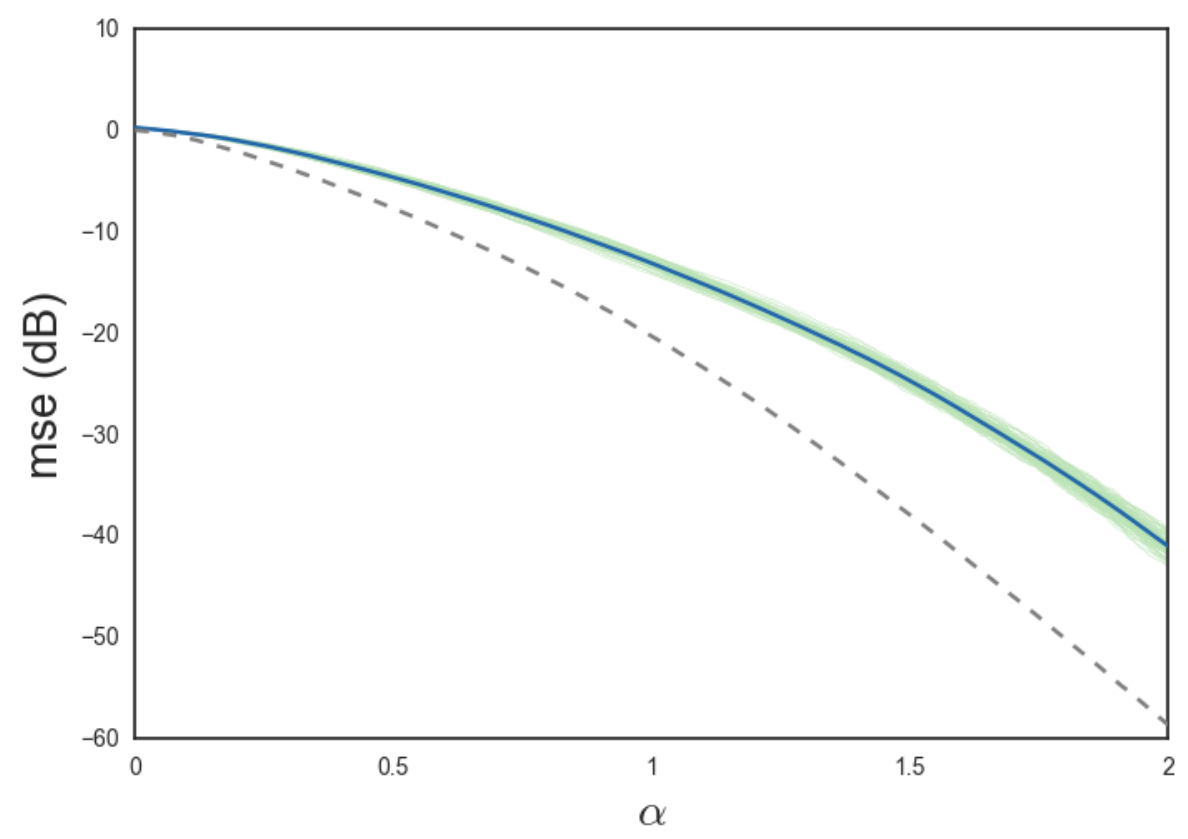

Figure 4.1: Comparison of the normalized reconstruction error with the Bayesian Online CS algorithm in two noiseless standard CS scenarios: with prior identical to the signal generating distribution (dashed) $\phi(x) \equiv \phi_{0}(x)=(1-0.1) \delta(x)+0.1 \mathcal{N}(0,1)$, and with a mismatched prior (green: 100 simulations; blue: mean) $\phi(x)=(1-0.4) \delta(x)+0.4 \mathcal{N}(0.2,0.6)$.

In this chapter two separate strategies to tackle this limitation are proposed. Using the Bayesian Online Compressed Sensing framework as a canvas, they both consist in the addition of extra steps in Algorithm 1 for the learning of hyperparameters in the prior distribution. Nevertheless, since the starting points and theoretical basis of both strategies are different, they are presented here in two 


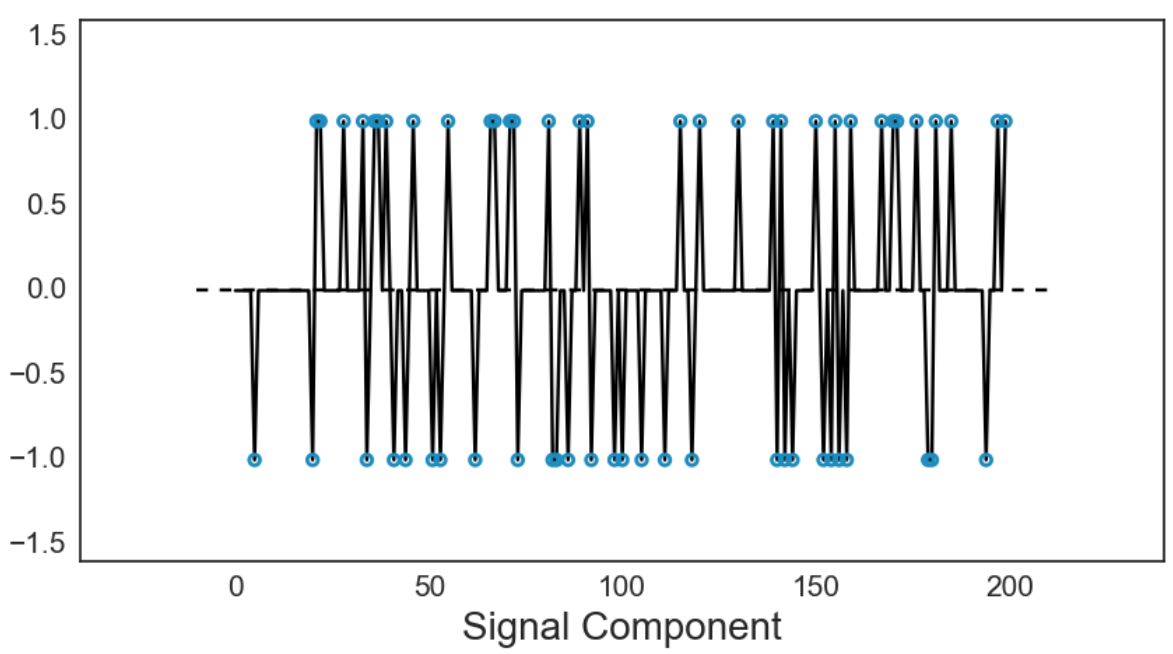

(a) $\phi(x)=\phi_{0}(x)$

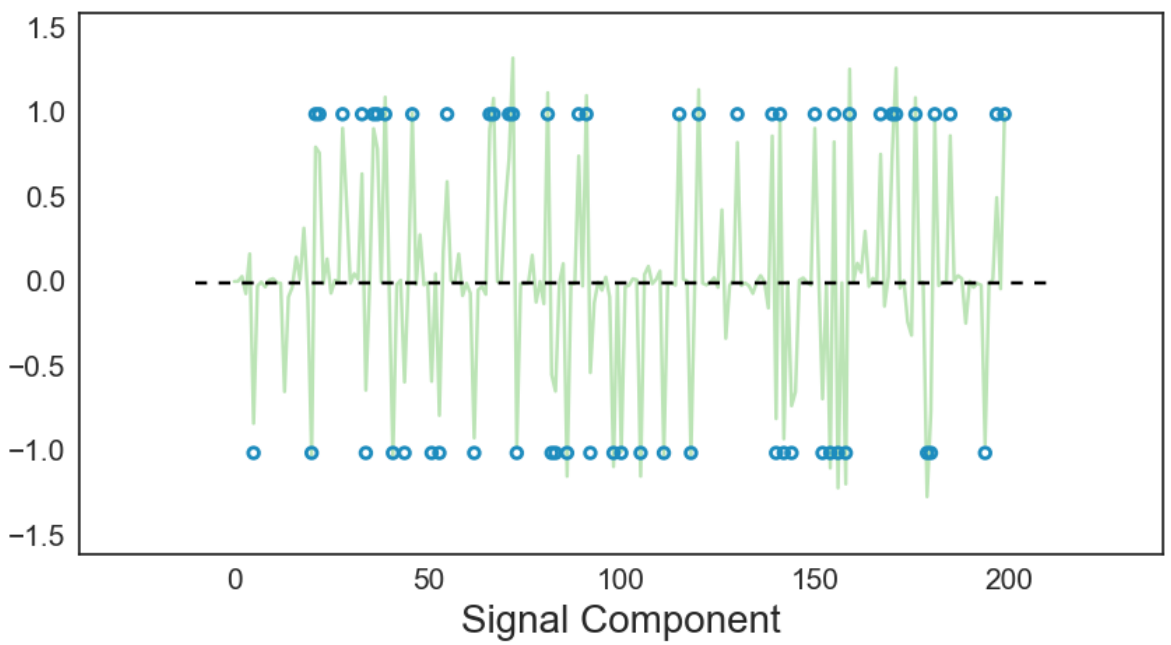

(b) $\phi(x) \neq \phi_{0}(x)$

Figure 4.2: Comparison between the reconstructed signals with the Bayesian Online CS algorithm in two noiseless standard CS scenarios: (a) prior identical to the signal generating distribution $\phi(x) \equiv \phi_{0}(x)=(1-\rho) \delta(x)+\rho[\delta(x+1) / 2+\delta(x-2) / 2]$, and (b) mismatched prior $\phi(x)=(1-\rho) \delta(x)+\rho \mathcal{N}(0,1)$. In both cases, $\rho=0.3$ and $\alpha=2$. The true signal $\boldsymbol{x}^{0}$ is shown in blue circles and the respective reconstructions in colored lines.

separate sections. In the first one, the optimal parameters are learned through extremization of the free-energy, defined as the logarithm of the evidence. This technique has already been proven successful in the offline Bayesian Compressed Sensing setting, but here its viability in the online scenario is shown. The second strategy is based on a Bayesian formulation of the $\ell_{1}$-minimization problem (i.e. the LASSO), where the sparsity parameter is learned through minimization of the KL-divergence. In what follows, we will consider that all signals are generated by the distribution $\phi_{0}(x)=(1-\rho) \delta(x)+\rho g(x)$, for some distribution $g(x)$ with no finite mass at the origin. 


\subsection{PARAMETER LEARNING}

Consider first the prior

$$
\phi(x)=\left(1-\rho^{\prime}\right) \delta(x)+\rho^{\prime} s(x),
$$

where $\rho^{\prime} \neq \rho$ and $s(x) \equiv s(x ; \boldsymbol{\theta})$ may have the same functional form as $g(x)$ but the wrong parameters, or it may be of an entirely different type. In any case, $s(x) \neq g(x)$. It will be shown that a free-energy-like quantity can be minimized with respect to $\rho^{\prime}$ and all other parameters in $s(x)$ at every iteration in order to find an optimal prior distribution, i.e. the best approximation to $\phi_{0}(x)$.

In this section we will introduce a technique which has already been applied successfully to the Bayesian offline Compressed Sensing framework [74, 75]. Assume for now that the prior distribution $\phi(x)=\phi(x ; \boldsymbol{\theta})$ is parameterized by the set of hyperparameters $\boldsymbol{\theta}=\left(\theta_{i}\right)$. For example, if the prior is the Gauss-Bernoulli distribution $\phi(x)=(1-\rho) \delta(x)+\rho / \sqrt{2 \pi \sigma^{2}} \exp \left[-(x-\bar{x})^{2} / 2 \sigma^{2}\right]$, then $\boldsymbol{\theta}=\left(\rho, \bar{x}, \sigma^{2}\right)$ and we write $\phi(x)=\phi\left(x ; \rho, \bar{x}, \sigma^{2}\right)$. Now consider that the signal generating distribution is of the same type, but parameterized by $\boldsymbol{\theta}_{0}$ (the Gauss-Bernoulli case would correspond to $\left.\phi_{0}(x)=\phi\left(x ; \rho_{0}, \bar{x}_{0}, \sigma_{0}^{2}\right)\right)$. Whatever the set $\boldsymbol{\theta}$ we make use of in the inference process, after $t$ measurements the full posterior distribution (3.3) can be rewritten

$$
P\left(\boldsymbol{x} \mid D^{t} ; \boldsymbol{\theta}\right)=\frac{P\left(D^{t} \mid \boldsymbol{x}\right) \phi(\boldsymbol{x} ; \boldsymbol{\theta})}{\int d \boldsymbol{x}^{\prime} P\left(D^{t} \mid \boldsymbol{x}^{\prime}\right) \phi\left(\boldsymbol{x}^{\prime} ; \boldsymbol{\theta}\right)} .
$$

Consider the joint distribution $P\left(\boldsymbol{x}, D^{t} ; \boldsymbol{\theta}\right)=P\left(D^{t} \mid \boldsymbol{x}\right) \phi(\boldsymbol{x} ; \boldsymbol{\theta})$ and its marginal

$$
P\left(D^{t} ; \boldsymbol{\theta}\right) \equiv \int d \boldsymbol{x} P\left(D^{t} \mid \boldsymbol{x}\right) \phi(\boldsymbol{x} ; \boldsymbol{\theta}) .
$$

The KL-divergence between the two marginals $P\left(D^{t} ; \boldsymbol{\theta}\right)$ and $P\left(D^{t} ; \boldsymbol{\theta}_{0}\right)$ is given by $D_{K L}\left(P_{\boldsymbol{\theta}_{0}} \| P_{\boldsymbol{\theta}}\right)=\int d D^{t} P\left(D^{t} ; \boldsymbol{\theta}_{0}\right) \log \left[P\left(D^{t} ; \boldsymbol{\theta}_{0}\right) / P\left(D^{t} ; \boldsymbol{\theta}\right)\right] \geq 0$. Inversion of the logarithm term results in $\int d D^{t} P\left(D^{t} ; \boldsymbol{\theta}_{0}\right) \log \left[P\left(D^{t} ; \boldsymbol{\theta}_{0}\right) / P\left(D^{t} ; \boldsymbol{\theta}_{0}\right)\right] \leq 0$ or, by making the integral implicit,

$$
\left\langle\log \frac{\int d \boldsymbol{x} P\left(D^{t} \mid \boldsymbol{x}\right) \phi\left(\boldsymbol{x} ; \boldsymbol{\theta}_{0}\right)}{\int d \boldsymbol{x} P\left(D^{t} \mid \boldsymbol{x}\right) \phi(\boldsymbol{x} ; \boldsymbol{\theta})}\right\rangle_{P\left(D^{t} ; \boldsymbol{\theta}_{0}\right)} \leq 0 .
$$

Now let us define the free-energy-like function

$$
F(\boldsymbol{\theta}):=-\log \int d \boldsymbol{x} P\left(D^{t} \mid \boldsymbol{x}\right) \phi(\boldsymbol{x} ; \boldsymbol{\theta}) .
$$


What equation (4.4) tells us is that

$$
\left\langle F\left(\boldsymbol{\theta}_{0}\right)\right\rangle_{P\left(D^{t} ; \boldsymbol{\theta}_{0}\right)} \leq\langle F(\boldsymbol{\theta})\rangle_{P\left(D^{t} ; \boldsymbol{\theta}_{0}\right)}
$$

for any values of $\boldsymbol{\theta}$. As a consequence, ${ }^{1}$ the derivatives of $F$ by the components of $\boldsymbol{\theta}$ should be zero at $\boldsymbol{\theta}=\boldsymbol{\theta}_{0}$ if $F(\boldsymbol{\theta})$ is sufficiently smooth. That is, for $\boldsymbol{\theta}=\left(\theta_{i}\right)$,

$$
\left\langle\left.\left.\frac{\partial}{\partial \theta_{i}} F(\boldsymbol{\theta})\right|_{P\left(D^{t} ; \boldsymbol{\theta}_{0}\right)}\right|_{\theta_{i}=\theta_{0, i}}=0 .\right.
$$

In summary, this means that in the signal inference process with a mismatched prior there is a quantity $F$ which is at its minimum when the "correct" hyper-parameters (i.e. hyper-parameters that match the ones at the actual generating distribution) are used. This leads to a simple method for the estimation of optimal parameters $\hat{\boldsymbol{\theta}}$ :

$$
\hat{\boldsymbol{\theta}}=\underset{\boldsymbol{\theta}}{\arg \min } F(\boldsymbol{\theta}) .
$$

Since the integral in (4.3) is the evidence (i.e. the denominator) in the Bayes' formulation of the Compressed Sensing problem (4.2), this procedure is often called maximization of evidence (e.g. [78]). ${ }^{2}$ In one Bayesian offline Compressed Sensing application [75], the AMP algorithm (Appendix A) was adapted to perform the minimization of $F(\boldsymbol{\theta})$ at every message's iteration, in a procedure similar to an Expectation-Maximization algorithm [4]. Here we build on this idea and propose that updating the hyper-parameters $\boldsymbol{\theta}$ according to (4.8) after every new measurement, but otherwise keeping the Bayesian online Compressed Sensing algorithm intact, leads to optimal $\boldsymbol{\theta}$. In particular, if the prior has the same functional form as the generating distribution, then $\boldsymbol{\theta} \rightarrow \boldsymbol{\theta}_{0}$.

As a practical example, consider the ever-present case of the Gauss-Bernoulli prior $\phi\left(\boldsymbol{x} ; \rho, \sigma^{2}, \bar{x}\right)=\prod_{i=1}^{N} \phi\left(x_{i} ; \rho, \sigma^{2}, \bar{x}\right)$, where each factor in the product is defined by

$$
\phi\left(x_{i} ; \rho, \sigma^{2}, \bar{x}\right)=(1-\rho) \delta\left(x_{i}\right)+\frac{\rho}{\sqrt{2 \pi \sigma^{2}}} \exp \left[-\left(x_{i}-\bar{x}\right)^{2} /\left(2 \sigma^{2}\right)\right] .
$$

The signal generating distribution is then $\phi_{0}(\boldsymbol{x})=\phi\left(\boldsymbol{x} ; \rho_{0}, \sigma_{0}^{2}, \bar{x}_{0}\right)$. After $t$ measurements, equation (4.8) indicates that the best estimates $\hat{\rho}^{t}, \hat{\sigma}^{t}$ and $\hat{\bar{x}}^{t}$ of the hyperparameters $\rho_{0}, \sigma_{0}$ and $\bar{x}_{0}$ are given by the three equations $\hat{\rho}^{t}=\arg \min _{\rho} F\left(D^{t} ; \rho, \sigma, \bar{x}\right)$,

\footnotetext{
${ }^{1}$ To avoid complications, we assume that all parameters are defined over open intervals. For example, in the Gauss-Bernoulli distribution $\phi\left(x ; \rho, \bar{x}, \sigma^{2}\right)=(1-\rho) \delta(x)+\rho \mathcal{N}\left(\bar{x}, \sigma^{2}\right), \rho \in(0,1)$, $\sigma^{2} \in(0, \infty)$ and $\bar{x} \in(-\infty, \infty)$.

${ }^{2}$ Other names include maximization of type II likelihood [10], minimization of ABIC [1] and minimization of the Bethe free entropy [75].
} 
$\hat{\sigma}^{t}=\arg \min _{\sigma} F\left(D^{t} ; \rho, \sigma, \bar{x}\right)$ and $\hat{\bar{x}}^{t}=\arg \min _{\bar{x}} F\left(D^{t} ; \rho, \sigma, \bar{x}\right)$. The solutions to this system of equations can be found through explicit calculation of the quantity (4.8) and its derivation by $\rho, \sigma$ and $\bar{x}$. At the minimum, its derivatives must be zero (equation (4.7)), i.e. $\left.(\partial / \partial \rho) F\left(D^{t} ; \rho, \sigma, \bar{x}\right)\right|_{\rho=\hat{\rho}^{t}}=0$, and similar expressions for the other variables. These equations can be reduced to the search for the fixed points of the following system:

$$
\left\{\begin{array}{l}
\rho^{t}=\frac{\sum_{i}\left(\frac{1+a_{i}^{t}\left(\sigma^{t}\right)^{2}}{h_{i}^{t}+\bar{x}^{t} /\left(\sigma^{t}\right)^{2}}\right) m_{i}^{t}}{\sum_{i}\left[1-\rho^{t}+\frac{\rho^{t}}{\sqrt{1+a_{i}^{t}\left(\sigma^{t}\right)^{2}}} E_{i}\left(\bar{x}^{t}, \sigma^{t}\right)\right]^{-1}} \\
\bar{x}^{t}=\frac{\sum_{i} m_{i}^{t}}{\rho^{t} \sum_{i}\left[\left(1-\rho^{t}\right) \sqrt{1+a_{i}^{t}\left(\sigma^{t}\right)^{2}} E_{i}\left(\bar{x}^{t}, \sigma^{t}\right)^{-1}+\rho^{t}\right]^{-1}} \\
\left(\sigma^{t}\right)^{2}=\frac{\sum_{i} v_{i}^{t}+\left(m_{i}^{t}\right)^{2}}{\rho^{t} \sum_{i}\left[\left(1-\rho^{t}\right) \sqrt{1+a_{i}^{t}\left(\sigma^{t}\right)^{2}} E_{i}\left(\bar{x}^{t}, \sigma^{t}\right)^{-1}+\rho^{t}\right]^{-1}}-\left(\bar{x}^{t}\right)^{2},
\end{array}\right.
$$

where $E_{i}(\bar{x}, \sigma) \equiv E_{i}\left(\bar{x}, \sigma ; a_{i}^{t}, h_{i}^{t}\right):=\exp \left[-\bar{x}^{2} /\left(2 \sigma^{2}\right)+\left(h_{i}^{t}+\bar{x} / \sigma^{2}\right)^{2} /\left(2\left(a_{i}^{t}+1 / \sigma^{2}\right)\right)\right]$. For all the examples below, these fixed point solutions were found iteratively. Equations (4.10) were transformed into update equations which could be calculated one at a time. The introduction of damping parameters $\gamma_{\rho}, \gamma_{\sigma}, \gamma_{\bar{x}}$ provide stability for the algorithm. The actual equations in this case become:

$$
\begin{aligned}
\hat{\rho}^{t+1}=\left(1-\gamma_{\rho}\right) \hat{\rho}^{t}+\gamma_{\rho} \frac{\sum_{i}\left(\frac{1+a_{i}^{t}\left(\sigma^{t}\right)^{2}}{h_{i}^{t}+\bar{x}^{t} /\left(\sigma^{t}\right)^{2}}\right) m_{i}^{t}}{\sum_{i}\left[1-\rho^{t}+\frac{\rho^{t}}{\sqrt{1+a_{i}^{t}\left(\sigma^{t}\right)^{2}}} E_{i}\left(\bar{x}^{t}, \sigma^{t}\right)\right]^{-1}} \\
\hat{\bar{x}}^{t+1}=\left(1-\gamma_{\bar{x}}\right) \hat{\bar{x}}^{t}+\gamma_{\bar{x}} \frac{\sum_{i} m_{i}^{t}}{\rho^{t+1} \sum_{i}\left[\left(1-\rho^{t+1}\right) \sqrt{1+a_{i}^{t}\left(\sigma^{t}\right)^{2}} E_{i}\left(\bar{x}^{t}, \sigma^{t}\right)^{-1}+\rho^{t+1}\right]^{-1}} \\
\left(\hat{\sigma}^{t+1}\right)^{2}=\left(1-\gamma_{\sigma}\right)\left(\hat{\sigma}^{t}\right)^{2} \\
+\gamma_{\sigma} \frac{\sum_{i} v_{i}^{t}+\left(m_{i}^{t}\right)^{2}}{\rho^{t+1} \sum_{i}\left[\left(1-\rho^{t+1}\right) \sqrt{1+a_{i}^{t}\left(\sigma^{t}\right)^{2}} E_{i}\left(\bar{x}^{t+1}, \sigma^{t}\right)^{-1}+\rho^{t+1}\right]^{-1}}-\left(\bar{x}^{t+1}\right)^{2} .
\end{aligned}
$$

These three equations together compose an additional step to be inserted in the original Algorithm 1. After update of the natural parameters $\left\{\left(a_{i}^{t}, h_{i}^{t}\right)\right\}$ and calculation of the means and variances of all components, the prior should also be updated in accord with the information obtained with the new measurement. We call this sequence the Bayesian Online Compressed Sensing Algorithm with Parameter Learning, and all its steps are summarized in Algorithm 2. 


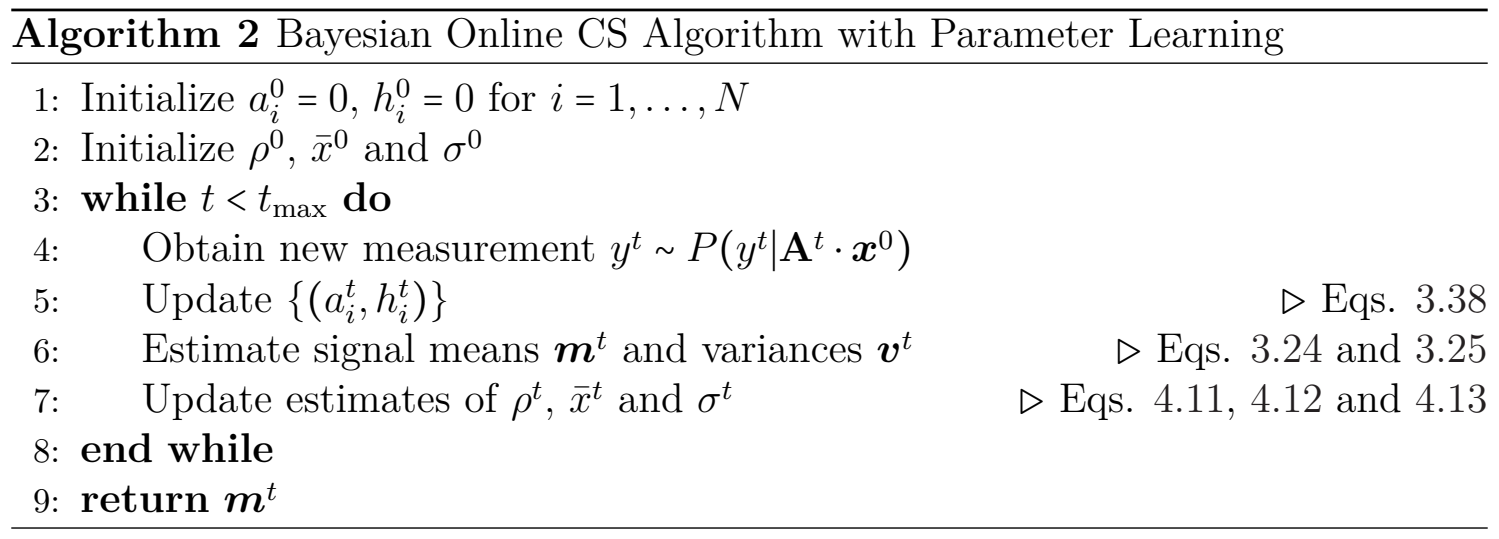

Figures 4.3 and 4.4 show the behavior of the algorithm, especially in what concerns equations (4.11), (4.12) and (4.13). A total of 100 simulations of the standard Compressed Sensing setting for each scenario can be visualized in the four panels of each figure. In all the examples, the simulations started with the same GaussBernoulli prior distribution $\phi\left(x ; \rho=0.4, \bar{x}=0.2, \sigma^{2}=0.6\right)$. This prior is of the same functional form as the signal generating distribution $\phi_{0}(x)=\phi\left(x ; \rho=0.1, \bar{x}=0, \sigma^{2}=\right.$ $1)$, but all its parameters are mismatched. In both examples it is clear that the parameters can converge to their true value, in average, even for $\alpha \ll 1$, provided appropriate dumping parameters. Many values were tested and the results shown were obtained with $\gamma_{\rho}=0.1, \gamma_{\sigma}=0.1$ and $\gamma_{\bar{x}}=0.01$.

Nevertheless, it is important to notice that knowledge of the true distribution might not be available, so that although these simulations show the possibility of hyper-parameter learning, the practical utility of the algorithm is still not guaranteed. What is a remarkable result, though, is that the algorithm performs well even when the functional form of the generating distribution is unknown. Figures 4.5 and 4.6 demonstrate a learning scenario where signals are generated by the ternary distribution $\phi_{0}(x)=\left(1-\rho_{0}\right) \delta(x)+\left(\rho_{0} / 2\right)[\delta(x+1)+\delta(x-1)]$, which generates null components with probability $\rho$, and $+1 /-1$ components with probability $\rho / 2$ each. In these examples, the Gauss-Bernoulli prior distribution was used, which not only is of a different functional form but also possesses a number of parameters different from the ternary distribution. The figures show that parameter learning is possible even in this scenario, with $\rho$ converging to $\rho_{0}$ and the other parameters $\hat{\bar{x}}^{t}$ and $\left(\hat{\sigma}^{2}\right)^{t}$ converging for the mean and variance, respectively, of the distribution $g(x)=[\delta(x+1)+\delta(x-1)] / 2$. What remains an open question is what type of distributions make parameter learning possible and effective for Compressed Sensing. In this work we chose not to tackle this question and introduced the method and examples above as proof of the possibility of learning without perfect knowledge of the signal generating distribution. 


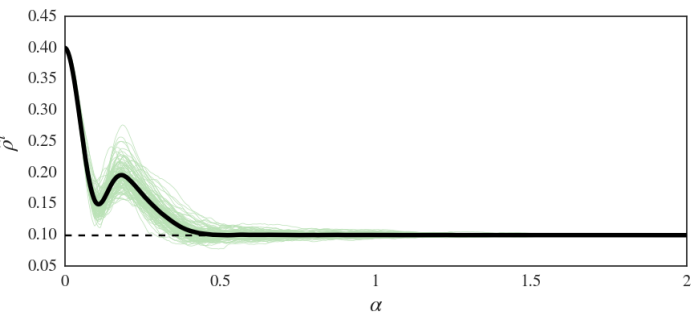

(a) $\hat{\rho}^{t}$

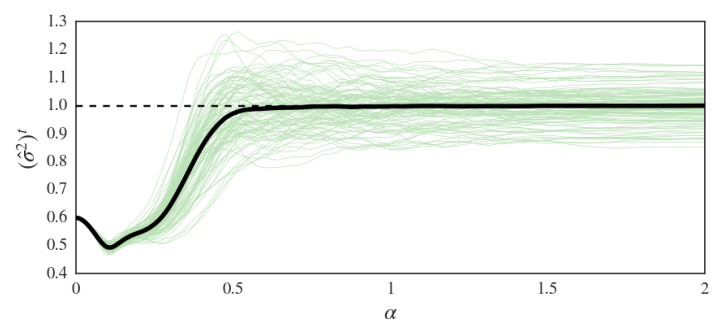

(c) $\hat{\sigma}^{t}$

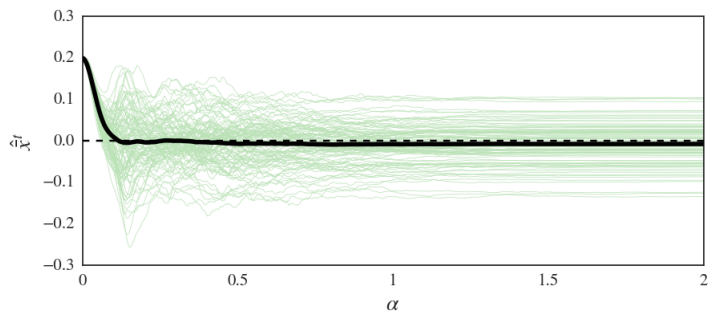

(b) $\hat{\bar{x}}^{t}$

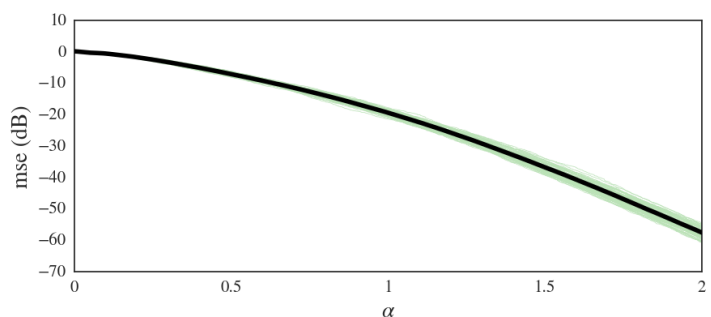

(d) Reconstruction error

Figure 4.3: Noiseless standard CS and parameter learning. Colored lines correspond to 100 individual simulations with $N=4000$, and black lines to their means. Dashed lines in the first 3 panels are the true parameter values in the generating distribution. Signal generating distribution: $\phi_{0}(x)=\left(1-\rho_{0}\right) \delta(x)+\rho_{0} \exp \left[-\left(x-\bar{x}_{0}\right)^{2} / 2 \sigma_{0}^{2}\right] / \sqrt{2 \pi \sigma_{0}^{2}}$, with $\rho_{0}=$ $0.1, \bar{x}_{0}=0$ and $\sigma_{0}^{2}=1$. Prior distribution: $\phi(x)=(1-\rho) \delta(x)+\rho \exp \left[-(x-\bar{x})^{2} / 2 \sigma^{2}\right] / \sqrt{2 \pi \sigma^{2}}$, with $\rho=0.4, \bar{x}=0.2$ and $\sigma^{2}=0.72$.

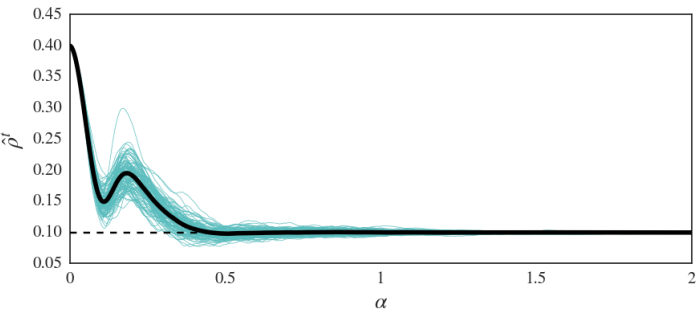

(a) $\hat{\rho}^{t}$

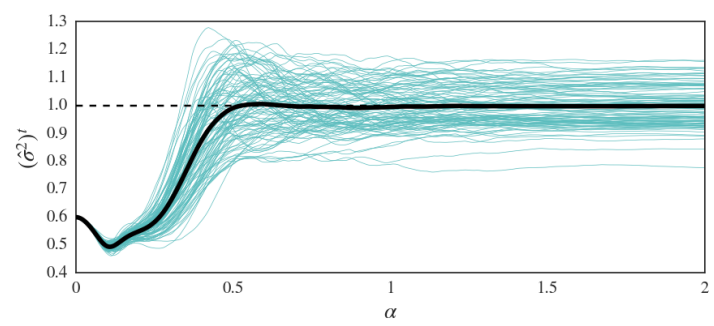

(c) $\hat{\sigma}^{t}$

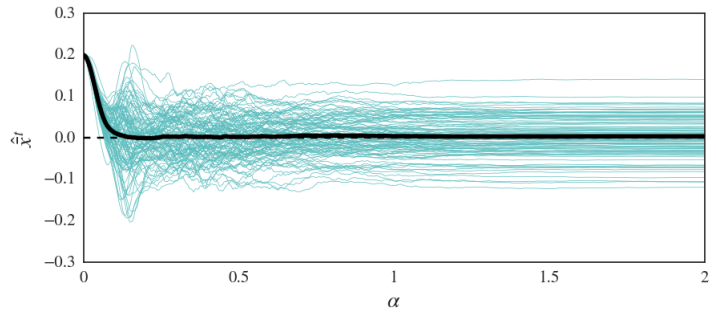

(b) $\hat{\bar{x}}^{t}$

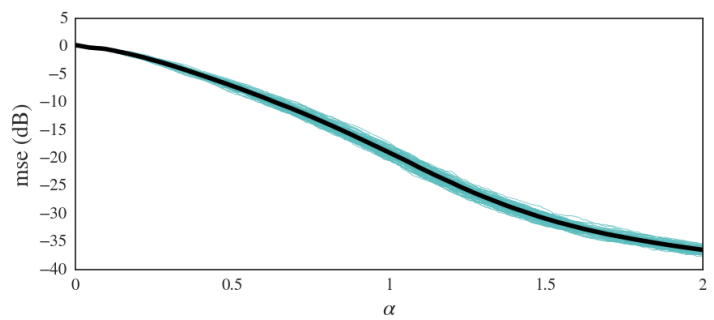

(d) Reconstruction error

Figure 4.4: Noisy standard CS and parameter learning. Colored lines correspond to 100 individual simulations with $N=4000$ and $\sigma_{n}^{2}=10^{-4}$, and black lines to their means. Dashed lines in the first 3 panels are the true parameter values in the generating distribution. Signal generating distribution: $\phi_{0}(x)=\left(1-\rho_{0}\right) \delta(x)+\rho_{0} \exp \left[-\left(x-\bar{x}_{0}\right)^{2} / 2 \sigma_{0}^{2}\right] / \sqrt{2 \pi \sigma_{0}^{2}}$, with $\rho_{0}=0.1, \bar{x}_{0}=0$ and $\sigma_{0}^{2}=1$. Prior distribution: $\phi(x)=(1-\rho) \delta(x)+\rho \exp [-(x-$ $\left.\bar{x})^{2} / 2 \sigma^{2}\right] / \sqrt{2 \pi \sigma^{2}}$, with $\rho=0.4, \bar{x}=0.2$ and $\sigma^{2}=0.72$. 


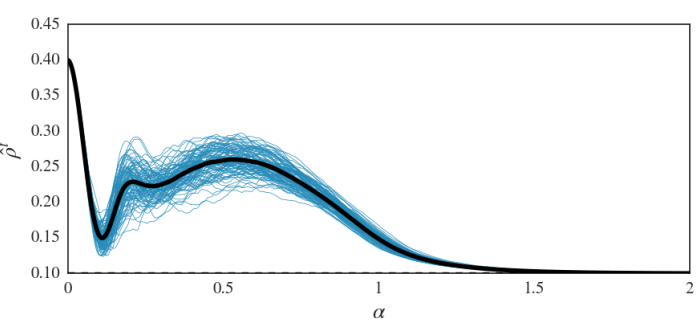

(a) $\hat{\rho}^{t}$

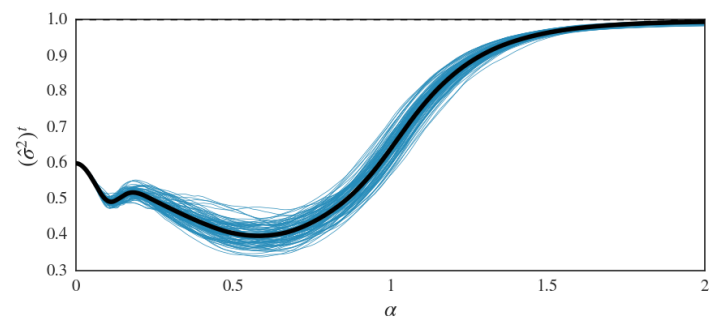

(c) $\hat{\sigma}^{t}$

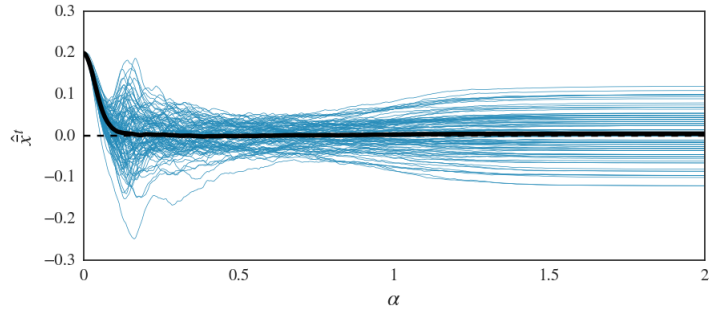

(b) $\hat{\bar{x}}^{t}$

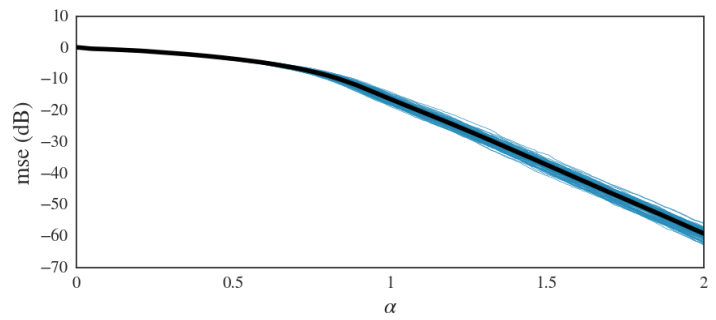

(d) Reconstruction error

Figure 4.5: Noiseless standard CS and parameter learning for prior with a mismatched function form. Colored lines correspond to 100 individual simulations with $N=4000$, and black lines to their means. Dashed lines in the first 3 panels are $\rho_{0}=0.1, \bar{x}_{0}=\int d x x g(x)=0$ and $\sigma_{0}^{2}=\int d x x^{2} g(x)-\bar{x}_{0}^{2}=1$, with $g(x)=\delta(x+1) / 2+\delta(x-1) / 2$. Signal generating distribution: $\phi_{0}(x)=\left(1-\rho_{0}\right) \delta(x)+\rho_{0} g(x)$. Prior distribution: $\phi(x)=$ $(1-\rho) \delta(x)+\rho \exp \left[-(x-\bar{x})^{2} / 2 \sigma^{2}\right] / \sqrt{2 \pi \sigma^{2}}$, with $\rho=0.4, \bar{x}=0.2$ and $\sigma^{2}=0.72$.

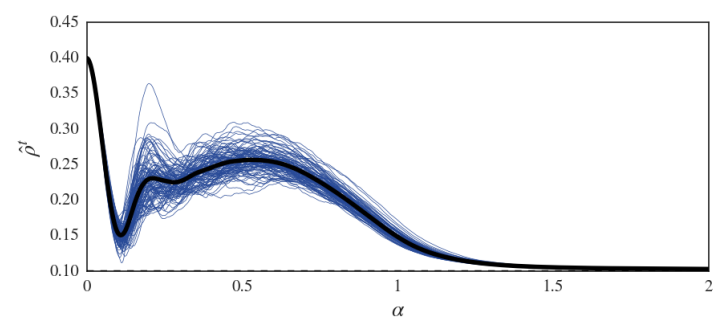

(a) $\hat{\rho}^{t}$

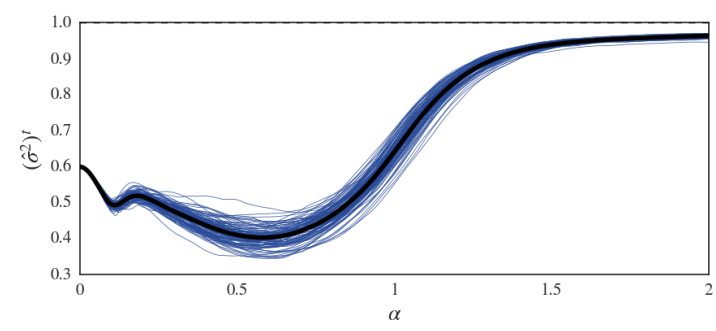

(c) $\hat{\sigma}^{t}$

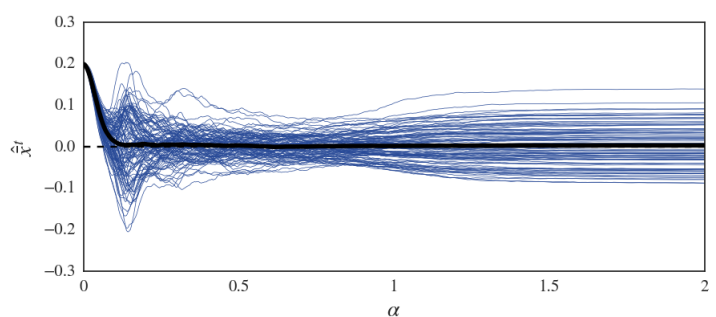

(b) $\hat{\bar{x}}^{t}$

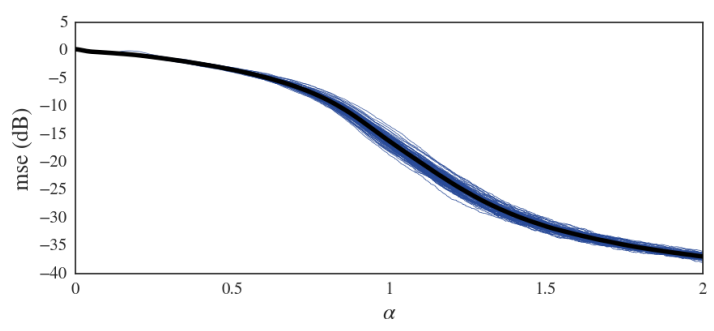

(d) Reconstruction error

Figure 4.6: Noisy standard CS and parameter learning for prior with a mismatched function form. Colored lines correspond to 100 individual simulations with $N=4000$ and $\sigma_{n}^{2}=10^{-4}$, and black lines to their means. Dashed lines in the first 3 panels are $\rho_{0}=0.1, \bar{x}_{0}=\int d x x g(x)=0$ and $\sigma_{0}^{2}=\int d x x^{2} g(x)-\bar{x}_{0}^{2}=1$, with $g(x)=\delta(x+1) / 2+\delta(x-1) / 2$. Signal generating distribution: $\phi_{0}(x)=\left(1-\rho_{0}\right) \delta(x)+\rho_{0} g(x)$. Prior distribution: $\phi(x)=(1-\rho) \delta(x)+\rho \exp \left[-(x-\bar{x})^{2} / 2 \sigma^{2}\right] / \sqrt{2 \pi \sigma^{2}}$, with $\rho=0.4, \bar{x}=0.2$ and $\sigma^{2}=0.72$. 


\section{$4.3 \ell_{1}$-MinimizATION ALGORITHM FOR BAYESIAN ON- LINE CS}

As mentioned in the beginning of this work, $\ell_{1}$-minimization schemes (e.g. the LASSO (1.10)) are by far the most popular techniques for Compressed Sensing signal reconstructions. Section 1.4 has shown in an example how different values of the sparsity parameter $\lambda$ affect the reconstructed model, and there an optimal value $\hat{\lambda}$ was found through cross-validation. In fact, finding the best value of the shrinkage parameter is a common practical issue when using the LASSO, since it is data-dependent and typically non-trivial. Nevertheless, its great influence on the reconstructed signal - there is a direct relation between $\lambda$ and the number of nonzero coefficients in the estimate -, makes it crucial to get accurate estimates of $\hat{\lambda}$. As mentioned before, usual strategies in offline scenarios include cross-validation procedures [120] or direct calculation of an ideal number of non-zero components by adding one at a time in a recursive manner [47]. Unfortunately these strategies are of little use in the present setting, where each measurement has to be used at most once and in an online manner.

Note that the LASSO estimator can also be written in a Bayesian manner as $\boldsymbol{m}^{t}=\int d \boldsymbol{x} \boldsymbol{x} P_{\ell_{1}}\left(\boldsymbol{x} \mid D^{t} ; \beta, \lambda\right)$, where

$$
P_{\ell_{1}}\left(\boldsymbol{x} \mid D^{t} ; \beta, \lambda\right)=\lim _{\beta \rightarrow \infty} Z_{\beta, \lambda}^{-1} \exp \left[-\beta\left(\frac{1}{2 \sigma_{n}^{2}} \sum_{\mu=1}^{t}\left(y^{\mu}-\boldsymbol{A}^{\mu} \cdot \boldsymbol{x}\right)^{2}+\lambda\|\boldsymbol{x}\|_{1}\right)\right],
$$

with $Z_{\beta, \lambda}$ a normalization factor dependent on $\beta$ and $\lambda$. It is from this formulation that the strategy presented in this section is drawn upon. Expression (4.14) suggests the introduction of the Laplace prior

$$
\phi(\boldsymbol{x} ; \lambda)=(2 / \lambda) \exp \left(-\lambda\|\boldsymbol{x}\|_{1}\right)
$$

in the mean-field posterior distribution (3.23), effectively altering the projection step of the Bayesian Online Compressed Sensing algorithm to the similar expression

$$
\widetilde{P}\left(\boldsymbol{x} \mid D^{t} ; \lambda\right) \simeq \prod_{i=1}^{N}\left(\frac{e^{-a_{i}^{t} x_{i}^{2} / 2+h_{i}^{t} x_{i}-\lambda\left|x_{i}\right|}}{Z\left(a_{i}^{t}, h_{i}^{t} ; \lambda\right)}\right)
$$

where $Z(a, h ; \lambda):=\int d x \exp \left(-a x^{2} / 2+h x-\lambda|x|\right)$ and, ideally, $\lambda=\hat{\lambda}$. The use of Laplace-like priors is not new in the literature $[61,97,54]$, but its introduction as part of an online algorithm is a novelty [106]. The proposal here, just like in the previous section, is to introduce an extra step in Algorithm 1 for the estimation of 
$\hat{\lambda}$, but otherwise to maintain its structure intact. Note that here also the natural parameters update equations (3.38) remain unchanged, since they are independent of choices of prior distributions. On the other hand, the means and variances become, respectively,

$$
\begin{aligned}
m_{i}^{t} & =\left(\partial / \partial h_{i}^{t}\right) \ln Z\left(a_{i}^{t}, h_{i}^{t} ; \lambda\right) \\
v_{i}^{t} & =\left(\partial^{2} /\left(\partial h_{i}^{t}\right)^{2}\right) \ln Z\left(a_{i}^{t}, h_{i}^{t} ; \lambda\right),
\end{aligned}
$$

paralleling equations (3.24) and (3.25). Below it is devised a learning scheme for $\hat{\lambda}$ to occur concurrent with the measurements.

Consider as a representative example the update equations (3.38) for the noisy standard CS scenario, which can be explicitly written as $a_{i}^{t+1}=a_{i}^{t}+\left(A_{i}^{t+1}\right)^{2} /\left(\sigma_{n}^{2}+\chi^{t+1}\right)$ and $h_{i}^{t+1}=h_{i}^{t}+A_{i}^{t+1}\left(y^{t+1}-\Delta^{t+1}\right) /\left(\sigma_{n}^{2}+\chi^{t+1}\right)+m_{i}^{t}\left(A_{i}^{t+1}\right)^{2} /\left(\sigma_{n}^{2}+\chi^{t+1}\right)$. Taking the expected value of $d a_{i}^{\mu}=a_{i}^{\mu}-a_{i}^{\mu-1}$ and $d h_{i}^{\mu}=h_{i}^{\mu}-h_{i}^{\mu-1}$ over the measurement vectors $\boldsymbol{A}^{\mu}$ results in

$$
\begin{aligned}
& \left\langle d a_{i}^{\mu}\right\rangle_{A^{\mu}} \simeq N^{-1} /\left(\sigma_{n}^{2}+\chi^{\mu}\right) \\
& \left\langle d h_{i}^{\mu}\right\rangle_{\boldsymbol{A}^{\mu}} \simeq N^{-1} m_{i}^{t} /\left(\sigma_{n}^{2}+\chi^{\mu}\right),
\end{aligned}
$$

which means all the natural parameters $a_{i}^{t}=\sum_{\mu=1}^{t} d a_{i}^{\mu}$ typically grow with more and more measurements (Figure 3.6a). Similarly, whenever $x_{0, i}$ is different from zero, any parameters $h_{i}^{t}=\sum_{\mu=1}^{t} d h_{i}^{\mu}$ exhibit a growth in absolute value lead by the $m_{i}$ factor (Figure 3.7a). For any fixed $\lambda$, these considerations mean that the regularizing effect of the prior $\phi(\boldsymbol{x}, \lambda)$ in (4.16) would vanish asymptotically in what would be a typical case of a prior being dominated by the likelihood. At the same time, the limit $\lambda \rightarrow \infty$ is undesirable, since the soft constraints in $\widetilde{P}_{\lambda}(\boldsymbol{x}) \equiv \widetilde{P}\left(\boldsymbol{x} \mid D^{t} ; \lambda\right)$ due to the finite value of the natural parameters $\left\{\left(a_{i}^{t}, h_{i}^{t}\right)\right\}$ would not be enough to prevent the prior distribution to completely dominate the entire approximate posterior, leading all signal estimates to zero. Thus, in order to keep the signal sparsity always in focus during the signal reconstruction, an optimal value for $\lambda$ should be a function of $\left\{\left(a_{i}^{t}, h_{i}^{t}\right)\right\}$ (i.e. a function of the data $\left.D^{t}\right)$. This section's proposal is that the optimal estimate for the shrinkage parameter is not static - indeed, we find that $\hat{\lambda} \equiv \hat{\lambda}^{t}$ is dependent on $t$.

According to Section 3.1, whenever a probability distribution $r(x)$ is of the exponential family, i.e. $r(x) \propto \sum_{k} \xi_{k} s_{k}(x)$, minimization of $D_{K L}(q \| r)$ between $r(x)$ and any other distribution $q(x)$ with respect to $\xi_{k}$ is equivalent to matching the moments $\int d x q(x) s_{k}(x)$ and $\int d x r(x) s_{k}(x)$. In particular, given the exact posterior 
distribution $P$ as defined in the L.H.S. of (3.3),

$$
P\left(\boldsymbol{x} \mid D^{t}\right)=\frac{P\left(D^{t} \mid \boldsymbol{x}\right) \phi_{0}(\boldsymbol{x})}{\int d \boldsymbol{x}^{\prime} P\left(D^{t} \mid \boldsymbol{x}^{\prime}\right) \phi_{0}\left(\boldsymbol{x}^{\prime}\right)},
$$

minimization of $D_{K L}\left(P \| \widetilde{P}_{\lambda}\right)$ with respect to $\lambda$, i.e. finding $\hat{\lambda}^{t}$ such that

$$
\hat{\lambda}^{t}=\underset{\lambda}{\arg \min } D_{K L}\left(P \| \widetilde{P}_{\lambda}\right) .
$$

is the same as finding the value of $\lambda$ which solves the implicit equation

$$
\langle|\boldsymbol{x}|\rangle_{P}=\langle|\boldsymbol{x}|\rangle_{\widetilde{P}_{\lambda}},
$$

where $\langle|\boldsymbol{x}|\rangle_{P}$ is the best possible estimate for the true sparsity of the original signal, $Q_{0} \equiv \int d x|x| \phi_{0}(x)$. Unfortunately the L.H.S. of expression (4.21) is generally unknown, but an argument can be made in defense of the approximation $\langle|\boldsymbol{x}|\rangle_{P} \simeq\left|\boldsymbol{m}^{t}\right|$. As more and more measurements are obtained and the approximate posterior distribution (4.16) concentrates around the true value of the signal, two limits are met: (a) $\langle|x|\rangle_{\widetilde{P}_{\lambda}} \underset{t \rightarrow \infty}{\longrightarrow}\langle|x|\rangle_{P}$ and (b) $\langle|x|\rangle_{\widetilde{P}_{\lambda}} \underset{t \rightarrow \infty}{\longrightarrow}\left|\langle x\rangle_{\widetilde{P}_{\lambda}}\right|$. The first limit has been proved in the large signal size limit $N \rightarrow \infty$ in [105] for an exact prior; here its validity is assumed also in the present scenario. As for the second limit, Minkowski inequality guarantees that $\langle|\boldsymbol{x}|\rangle_{\widetilde{P}_{\lambda}} \leq\left|\boldsymbol{m}^{t}\right|$; with the collapse of the marginal distribution $\widetilde{P}_{i}\left(x_{i}\right) \equiv \int d \boldsymbol{x}_{\backslash} \widetilde{P}\left(\boldsymbol{x} \mid D^{t} ; \lambda\right)$ around the true value $x_{0, i}$ at $t \rightarrow \infty$, no probability mass crosses the $x_{i}=0$ axis, so that the equality limit is met. Together, these results mean a good estimate for the solution of (4.21) is given by $\hat{\lambda}^{t}$ that solves

$$
\langle|\boldsymbol{x}|\rangle_{\widetilde{P}_{\lambda}} \simeq\left|\boldsymbol{m}^{t}\right|
$$

for $\lambda$, where the R.H.S. can be directly calculated from $\widetilde{P}\left(\boldsymbol{x} \mid D^{t} ; \hat{\lambda}^{t-1}\right)$. This implicit expression corresponds to an extra step in the online algorithm for the recovery of the signal, which is shown in full below (Algorithm 3). It should be noted that numerical simulations prove what could have been a reasonable assumption: in Figure 4.7 it is possible to see that in the early stages when only a few measurements have been obtained, even when the prior is exactly the same as the generative distribution, $\left|\boldsymbol{m}^{t}\right|$ is not an excellent approximation of $\langle|\boldsymbol{x}|\rangle_{P}$. But it is immediately clear that the approximation gets progressively better with more measurements. For this reason, we found that the use of a small damping factor $\gamma$ in the update of $\hat{\lambda}^{t}$ provides faster and more accurate learning.

Figure 4.8 shows the average normalized mean squared error over 100 runs of 

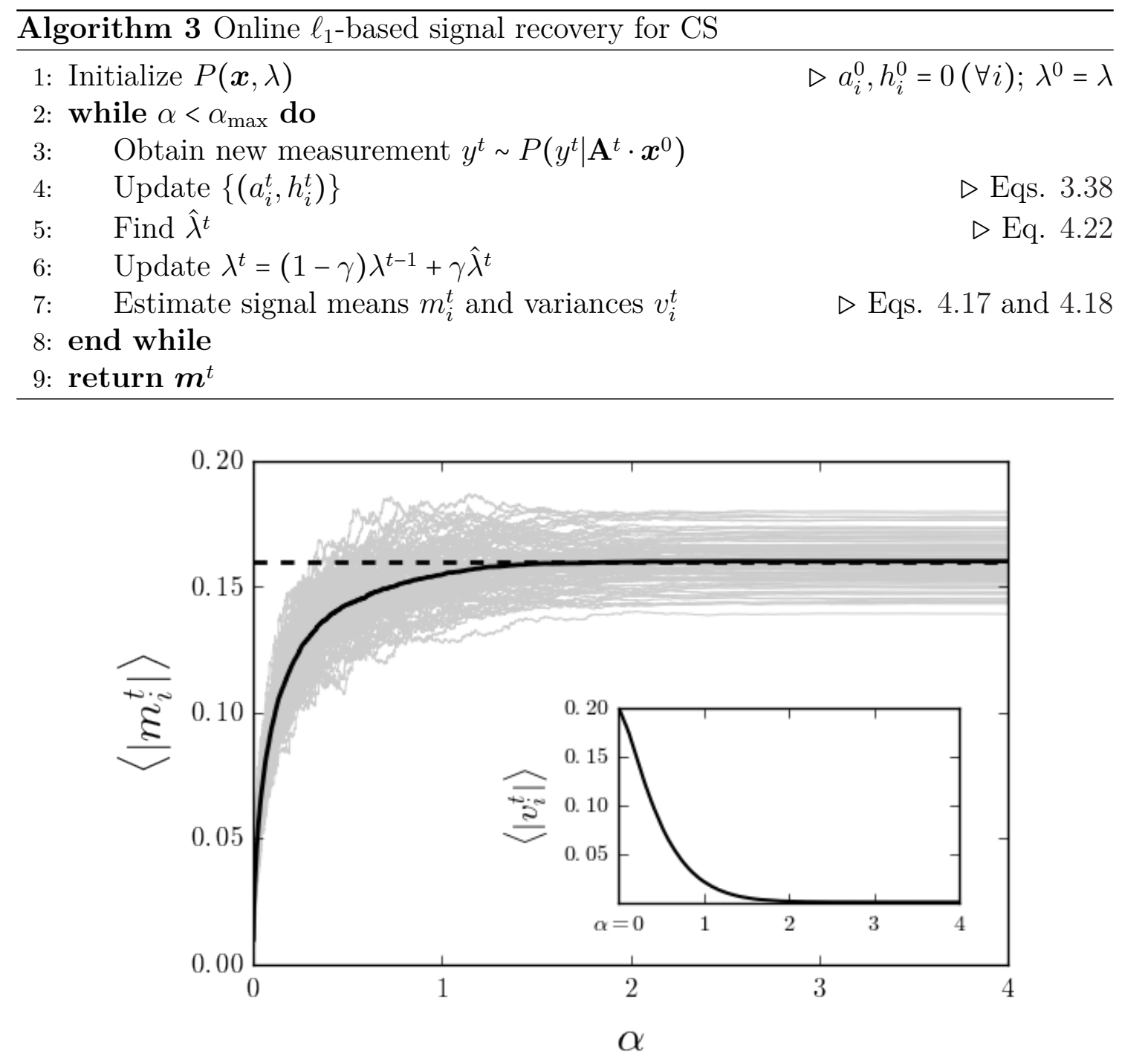

Figure 4.7: True signal sparsity $\left\langle\left|x_{i}^{0}\right|\right\rangle$ and approximation $\langle|x|\rangle_{\widetilde{P}}$ with exact prior $\phi(x) \equiv \phi_{0}(x)=(1-\rho) \delta(x)+(\rho / \sqrt{2 \pi}) \exp \left(-x^{2} / 2\right)$. Gray lines correspond to 100 individual simulations of the noisy standard CS scenario with $N=1000, \rho=0.2$ and $\sigma_{n}^{2}=10^{-4}$; full black line correspond to their average. Dashed line is the true sparsity of the signal $Q_{0}=\int d x_{0}\left|x_{0}\right| \phi\left(x_{0}\right)$. Inset: Mean of the average variance of all estimate components. The absolute value approximation gets progressively better with growing $\alpha$; in fact, its accuracy matches the diminishing of the variance. In this case, for $\alpha$ slightly larger than 1 the approximation is almost exact.

the Online $\ell_{1}$-based signal recovery algorithm with $\lambda$ learning. Two different priors were used: $\phi(x)=(1-\rho) \delta(x)+\rho g(x)$ with a Gaussian non-sparse part $g(x)=$ $(1 / \sqrt{2 \pi}) \exp \left(-x^{2} / 2\right)$ and a binary non-sparse part $g(x)=[\delta(x+1)+\delta(x-1)] / 2$. Results are shown for noisy and noiseless standard CS measurements. For a more precise benchmark, lines corresponding to an altered version of the algorithm where $Q_{0}$ is exactly known and therefore does not need to be inferred from the signal estimates were added to each plot. The results show the viability of the scheme 
presented here as a computationally cheap option for online reconstruction of signals generated by an unknown distribution. In the four scenarios presented in Figure 4.8, the error decays approximately exponentially with $\alpha$.
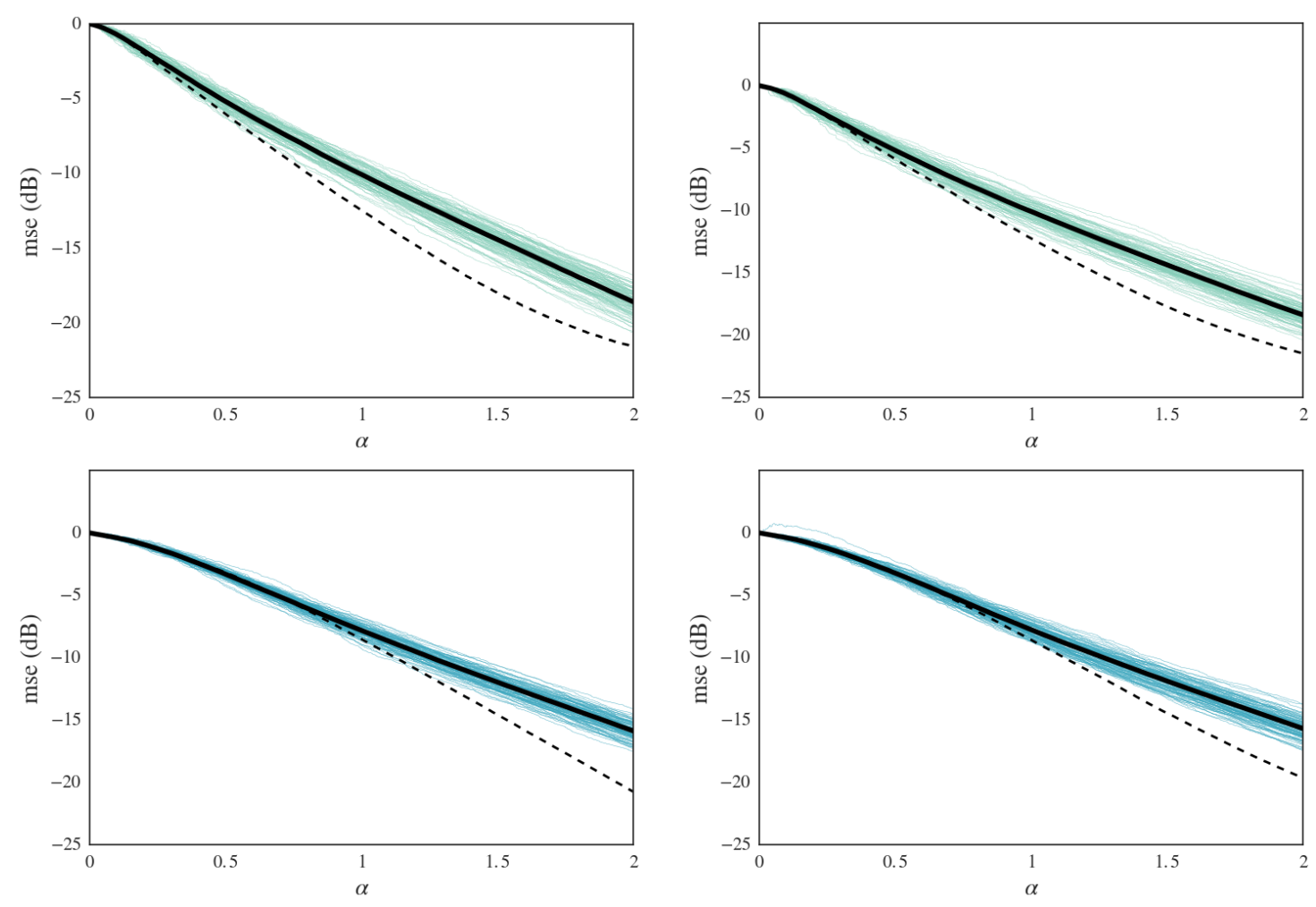

Figure 4.8: $\ell_{1}$-based reconstruction for CS with $\rho=0.1$. Figures (a) and $(b): g(x)=$ $(1 / \sqrt{2 \pi}) \exp \left(-x^{2} / 2\right)$. Figures (c) and $(d): g(x)=(\delta(x+1)+\delta(x-1)) / 2$. All lines correspond to the average of 100 simulations, with $Q_{0}$ known (dashed) or estimated through (4.22) (full). The top row consists of the noiseless standard CS scenario; the down row corresponds to the noisy scenario with $\sigma_{n}^{2}=10^{-4}$. For (a) and (b), all simulations with estimation of $Q_{0}$ were produced with $\gamma=10^{-3}$; for $(c)$ and $(d), \gamma=10^{-2}$.

The question might remain of how necessary the $\lambda$-learning step really is. To evaluate the answer in an accurate way, Figure 4.9 shows the normalized reconstruction error of many fixed values of $\lambda$ for the noiseless standard CS setting with a Gauss-Bernoulli prior. Figure 4.9a makes it clear that there is indeed an optimal value of fixed $\lambda$ that minimizes the reconstruction error. This value correspond to $\bar{\lambda}$ which minimizes the KL-divergence between the signal generating distribution $\phi_{0}(x)$ and the double-exponential $\phi(x ; \lambda) \propto \exp (-\lambda|x|)$. Nevertheless, Figure 4.9b demonstrates that even $\bar{\lambda}$ is sub-optimal - the reconstruction error of the scheme summarized in Algorithm 3 is always smaller, specially for large $\alpha$. This corroborates the remarks made earlier: $\hat{\lambda}^{t}$ has to typically increase with $\alpha$ in a scale similar to the other natural parameters in order to keep the sparsity in focus. In fact, this is exactly the empirically observed behavior (Figure 4.10). 


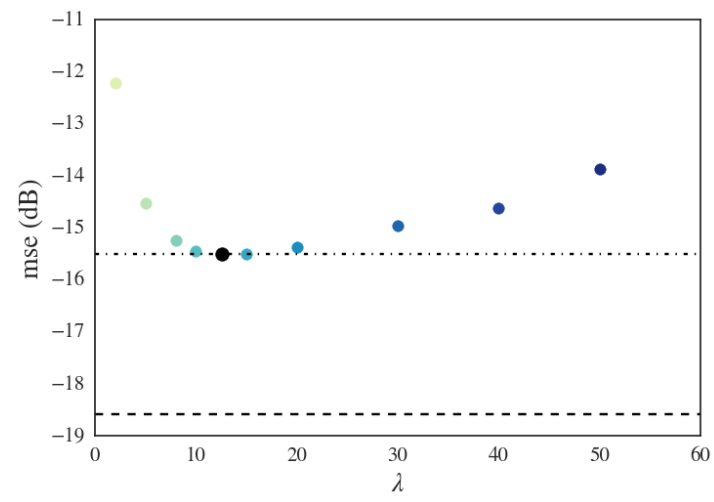

(a) $\alpha=2$

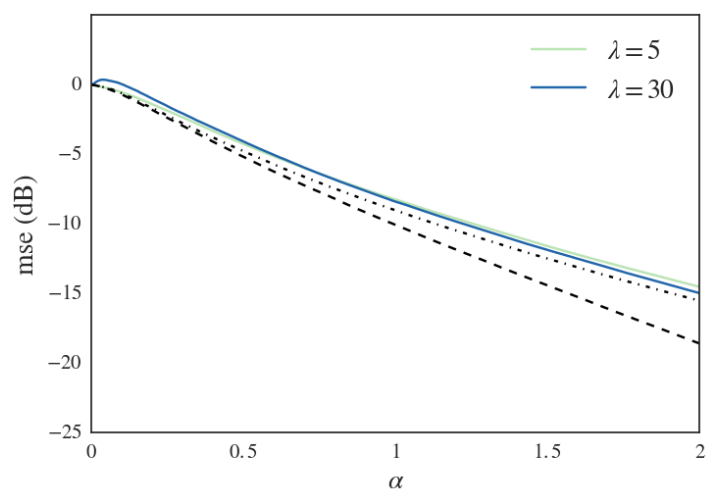

(b) Comparison with Algorithm 3

Figure 4.9: Fixed $\lambda$ : Bayesian online signal reconstruction for noiseless standard CS with Laplace prior $\phi(x ; \lambda) \propto \exp (-\lambda|x|)$ and signals generated from the Gauss-Bernoulli distribution $\phi_{0}(x)=(1-\rho) \delta(x)+\rho \mathcal{N}(0,1)$ with $\rho=0.1$. (a) Each point represents the average reconstruction error normalized by $Q_{0}$ of 100 simulations with $N=1000$ at $\alpha=$ 2. Black circle is the "optimal static value" $\bar{\lambda} \simeq 12.5$, whose error is also visible as the horizontal dash-dotted line. Horizontal dashed line is the reconstruction error obtained when $\lambda$ can be learned through Algorithm 3. (b) Reconstruction error for three different values of fixed $\lambda: \lambda=5$ (light green), $\lambda=30$ (blue) and $\lambda=\bar{\lambda}$ (dash-dotted), and for the reconstruction obtained with the Online $\ell_{1}$-based signal recovery algorithm for CS. All lines correspond to the average of 100 simulations with $N=1000$.
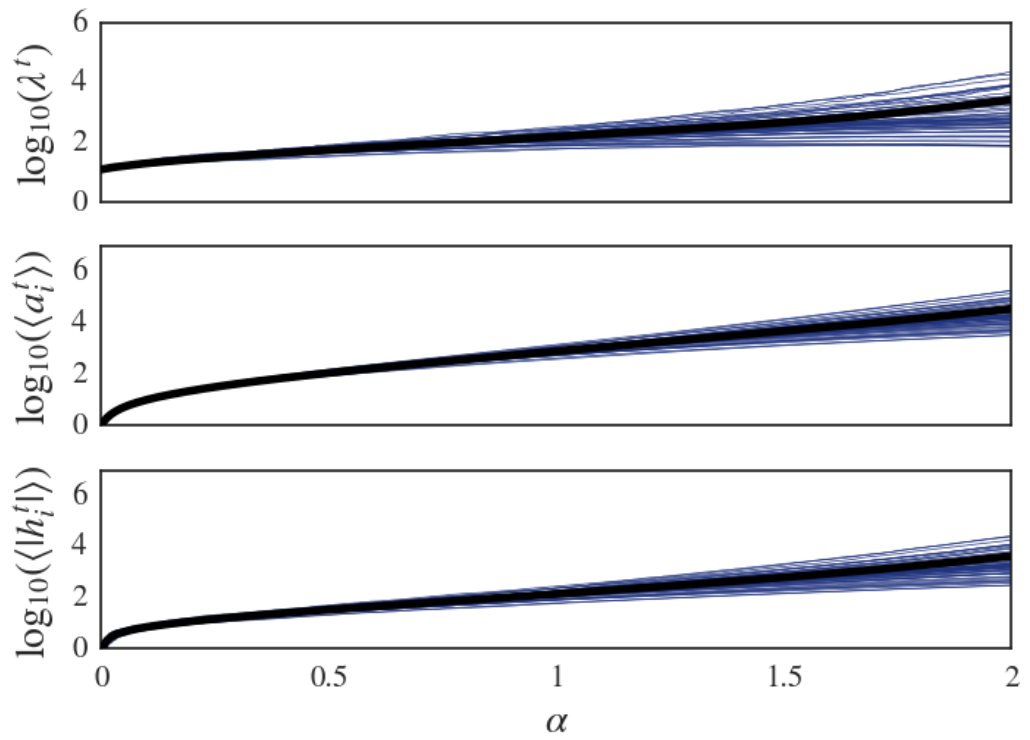

Figure 4.10: Growth scale of $\lambda^{t}$ in the Online $\ell_{1}$-based signal recovery algorithm for noiseless standard CS. Here, the signal generating distribution $\phi_{0}(x)=(1-\rho) \delta(x)+$ $\rho \mathcal{N}(0,1)$ with $\rho=0.1$ was completely known and therefore the solution to equation (4.21) was added in an ad-hoc manner. Each curve corresponds to the average of 50 simulations with $N=1000$ Note that $\lambda^{t}$, just like the natural parameters $\left\{\left(a_{i}^{t}, h_{i}^{t}\right)\right\}$, typically grows in an exponential manner in order to keep the reconstructed signal sparsity fixed. 



\section{CONCLUSION}

The study of the macroscopic behavior of many interacting components is at the core of Statistical Physics. With the employment of probabilistic and statistical tools, physicists have described the most varied physical systems with great success. More recently, it has become commonplace knowledge that the same techniques used in Theoretical Physics could be used in the analysis of a large variety of systems of diverse nature [24, 71, 130] — in particular, Information Systems [84, 93]. From the many works on the relation of the Physics of disordered systems and error-correcting codes $[127,129]$ to the more recent applications in Restricted Boltzmann machines [121], physicists have contributed to Information Theory novel ways in which to visualize classical problems.

This thesis has the ambition to bring even more insight into Compressed Sensing from a Statistical Physics point-of-view. Necessary and sufficient conditions for accurate signal reconstruction have typically been considered by the fields of Mathematics and Computer Science. But for very large signals, typical reconstruction characteristics can be analyzed using techniques such as the replica method. Even more than that, mean-field theories for disordered systems inspire the formulation of new techniques and algorithms.

We have shown here the viability of online learning in Compressed Sensing. Using a Bayesian framework, we have proposed a basic algorithm which performs similarly to offline techniques in the asymptotic regime and in the presence of noise. We have also introduced strategies to learn hyper-parameters using physical and information theoretical concepts. It was shown that priors can be optimized in order to best represent the signal generating distribution. Simulations and (sometimes extensive) calculations were used to argue for the truth of the claims here proposed. No doubt there is a lot more to be done - in real-life situations signals tend to present some kind of structural order $[41,56]$ and the full potential of online learning is only explored in the presence of time-varying signals [32, 128]. Both these scenarios have not been studied in this thesis and are clear future directions for the results presented here. In any case, we hope this work serves as a good introduction to the exciting theory of Compressed Sensing. 



\section{CHAPter A}

\section{Belief Propagation And the AMP ALGORITHM}

In order to derive the AMP algorithm for Compressed Sensing, we use this section to introduce in an instructional manner the Belief Propagation theory for efficient computation of marginal distributions and the particularly relevant formalism of factor graphs, well-suited for the message passing framework. ${ }^{1}$

\section{Factor Graphs and Message Passing}

Given any probability distribution $P\left(x_{1}, \ldots, x_{N}\right)$, the (in-)dependence relations between its variables can be represented in graph-like format. These representations are generally known as Graphical Models, which perhaps unsurprisingly borrow the terminology of graph theory. To keep the presentation short and relevant, we will avoid general definitions and introduce here only the terms deemed important to the understanding of what follows next.

Graphs $G$ consist of a set of nodes $V$ and a set of edges $E$. Edges connect nodes, i.e. each edge $e \in E$ is of the form $(v, w)$ with $v, w \in V$. Here we consider two restrictions: (1) $v \neq w$ always, which means no self-loops and (2) each pair of nodes is connected by at most one edge. In general, edges can be directed or undirected in the former case they point from one node to another. Graphs can also be bipartite, in which case $V$ can be divided into two subsets $V_{1}$ and $V_{2}$ such that $V=V_{1} \cup V_{2}$ and $V_{1} \cap V_{2}=\varnothing$, and every edge is of the form $\left(v_{1}, v_{2}\right)$ with $v_{1} \in V_{1}$ and $v_{2} \in V_{2}$. A (undirected) path is a sequence of nodes and edges $\left(v_{0}, e_{1}, v_{1}, e_{2}, v_{2}, \ldots, e_{k}, v_{k}\right)$ where edge $e_{i}$ connects nodes $v_{i-1}$ and $v_{i}$ and edges occur at most once. A path is also said to connect $v_{0}$ to $v_{k}$. A (undirected) cycle (or loop) is a path in which $v_{0}=v_{k}$ and no

\footnotetext{
${ }^{1}$ The content of this section is largely based on [4] and [84].
} 
other node occurs more than once. A connected graph is a graph where there is a path from every node $v$ to every node $w$, with $v, w \in V$. A tree is a connected graph with no cycles; alternatively, any two nodes in a tree are connected by exactly one path. Take the set of all shortest distances between all two nodes - the greatest value in this set is called the diameter of $G$.

Now consider the variables $\boldsymbol{x}=\left(x_{1}, \ldots, x_{N}\right)$ and their joint distribution factorized as

$$
P(\boldsymbol{x})=\frac{1}{Z} \prod_{a=1}^{M} \psi_{a}\left(x_{\partial a}\right)
$$

with $\partial a=\left\{i_{1}^{a}, \ldots, i_{k_{a}}^{a}\right\}$ being a subset of size $k_{a}$ of the set of indexes $\{1, \ldots, N\}$ and $x_{\partial a}=\left\{x_{i_{1}^{a}}, \ldots, x_{i_{a}^{a}}\right\}$. A factor graph for this distribution is a bipartite, undirected graph $^{2}$ built from two types of nodes: each factor $\psi_{a}$ is represented by a factor node, and each variable $x_{i}$ is represented by a variable node. Variable node $x_{j}$ is connected to factor $\psi_{b}$ by an edge if and only if $x_{j} \in \partial b$. As a bipartite graph, there are no edges between any two factor nodes or between any two variable nodes. We also define $\partial i$ as the set of function nodes which are connected to variable node $x_{i}$.

Let's exemplify this with two simple distributions. Consider first the four variable Markov chain (Figure A.1):

$$
P\left(x_{1}, x_{2}, x_{3}, x_{4}\right)=P\left(x_{1} \mid x_{2}\right) P\left(x_{2} \mid x_{3}\right) P\left(x_{3} \mid x_{4}\right) P\left(x_{4}\right) .
$$

with factors $\psi_{1}\left(x_{1}, x_{2}\right)=P\left(x_{1} \mid x_{2}\right), \psi_{2}\left(x_{2}, x_{3}\right)=P\left(x_{2} \mid x_{3}\right), \psi_{2}\left(x_{3}, x_{4}\right)=P\left(x_{3} \mid x_{4}\right)$ and $\psi_{4}\left(x_{4}\right)=P\left(x_{4}\right)$.

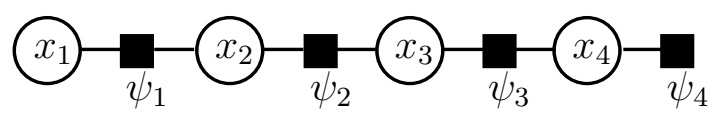

Figure A.1: Factor graph of a Markov chain

The second example is a distribution particularly relevant to the Statistical Physics community — the one-dimensional Ising model

$$
P(s)=\frac{1}{Z} \mathrm{e}^{-\beta \mathcal{H}(s)}, \quad \mathcal{H}(s)=-\sum_{i=1}^{N-1} s_{i} s_{i+1}-B \sum_{i=1}^{N} s_{i}
$$

with $s=\left(s_{1}, \ldots, s_{N}\right)$ and $s_{i} \in\{+1,-1\}$ (Figure A.2).

Consider the problem of calculating marginals for distributions like (A.1). In particular, let us take as an example the marginal $P\left(x_{1}\right) \equiv \sum_{x_{2}, x_{3}, x_{4}} P\left(x_{1}, x_{2}, x_{3}, x_{4}\right)$

\footnotetext{
${ }^{2}$ That is, each and every one of its edges are undirected.
} 


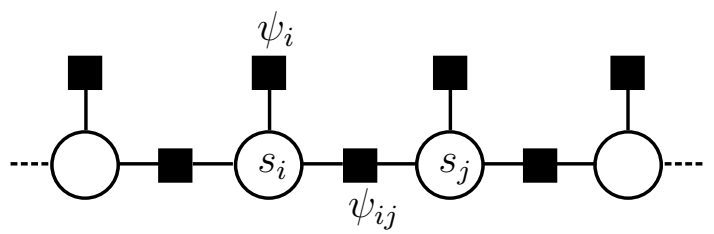

Figure A.2: Factor graph of the one-dimensional Ising model

in model (A.2). By definition,

$$
P\left(x_{1}\right)=\sum_{x_{2}, x_{3}, x_{4}} P\left(x_{1} \mid x_{2}\right) P\left(x_{2} \mid x_{3}\right) P\left(x_{3} \mid x_{4}\right) P\left(x_{4}\right),
$$

and moving the summation on $x_{4}$ to the far right results in

$$
P\left(x_{1}\right)=\sum_{x_{2}, x_{3}} P\left(x_{1} \mid x_{2}\right) P\left(x_{2} \mid x_{3}\right) \sum_{x_{4}} P\left(x_{3} \mid x_{4}\right) P\left(x_{4}\right) .
$$

The last summation, $\nu_{\psi_{3} \rightarrow x_{3}}\left(x_{3}\right) \equiv \sum_{x_{4}} P\left(x_{3} \mid x_{4}\right) P\left(x_{4}\right)$ can be understood as a message being transmitted by factor $\psi_{3}$ to the variable $x_{3}$. This message, on its own, is also composed by the simple message $\nu_{\psi_{4} \rightarrow x_{4}}\left(x_{4}\right)=P\left(x_{4}\right)$ which is transmitted by factor $\psi_{4}$ to the variable $x_{4}$. If we then shift the summation on $x_{3}$ to the right, we obtain

$$
P\left(x_{1}\right)=\sum_{x_{2}} P\left(x_{1} \mid x_{2}\right) \sum_{x_{3}} P\left(x_{2} \mid x_{3}\right) \nu_{\psi_{3} \rightarrow x_{3}}\left(x_{3}\right) .
$$

Here, we can define $\nu_{x_{3} \rightarrow \psi_{2}} \equiv P\left(x_{2} \mid x_{3}\right) \nu_{\psi_{3} \rightarrow x_{3}}\left(x_{3}\right)$ as a message from variable $x_{3}$ to the factor $\psi_{2}$, and $\nu_{\psi_{2} \rightarrow x_{2}}=\sum_{x_{3}} \nu_{x_{3} \rightarrow \psi_{2}}$ becomes a message from factor $\psi_{2}$ to the variable $x_{2}$. Therefore, following this procedure is equivalent to summarizing all information of the preceding nodes in an unique function which can be carried through the graph - this is known as message passing or belief propagation ${ }^{3}(\mathrm{BP})$. Imagine each variable $x_{i}$ could assume $\chi$ distinct values each. A naive approach to calculate the marginals here would consist of summing over all configurations, taking a time $O\left(\chi^{N}\right)$. The belief propagation procedure takes only $O(N)$.

To introduce the algorithm for general graphs, we start by defining the new variables $\nu_{i \rightarrow a}^{(t)}$ and $\hat{\nu}_{a \rightarrow i}^{(t)}$, which correspond to messages sent along edge $(i, a)$ from both directions at iteration $t$. Message $\nu_{i \rightarrow a}^{(t)}$ can be interpreted as the estimate at time $t$ of the marginal distribution of $x_{i}$ in a modified graphical model where the factor $a$ is non-existing. In other words, the marginal in the distribution $P(\boldsymbol{x})=$ $(1 / Z) \prod_{b \neq a} \psi_{b}\left(x_{\partial b}\right)$. Message $\hat{\nu}_{a \rightarrow i}^{(t)}$ has a similar interpretation - it is the estimate

\footnotetext{
${ }^{3}$ Alternative names come from different communities which have approached the problem from different angles. In Statistical Physics, this is called the Bethe-Peierls approximation and in coding theory this is the sum-product algorithm. Here we adopt belief propagation, which is the terminology of the artificial intelligence community.
} 
at time $t$ of the marginal distribution of $x_{i}$ in a modified graphical model where all factors in $\partial i$ except $a$ have been erased. With these definitions, the BP update rules are

$$
\begin{aligned}
& \nu_{i \rightarrow a}^{(t)}\left(x_{i}\right)=\prod_{b \in \partial i \backslash a} \nu_{b \rightarrow i}^{(t)}\left(x_{i}\right) \\
& \nu_{a \rightarrow i}^{(t)}\left(x_{i}\right)=\sum_{x_{\partial a \backslash i}} \psi_{a}\left(x_{\partial a}\right) \prod_{k \in \partial a \backslash i} \nu_{k \rightarrow a}^{(t)}\left(x_{k}\right),
\end{aligned}
$$

where $\partial a \backslash i$ is the set $\partial a$ with variable $i$ removed. At any moment, an estimate of the marginal $P\left(x_{i}\right)$ can be obtained through

$$
\nu_{i}^{(t)}\left(x_{i}\right) \propto \prod_{a \in \partial i} \nu_{a \rightarrow i}^{(t-1)}\left(x_{i}\right)
$$

For tree-like graphs with diameter $k^{*}$, it can be shown (Theorem 14.1 of [84]) that the BP equations converge to a fixed-point after at most $k^{*}$ interactions and that these fixed-point messages provide the exact marginals. But here in this work (and in CS in general) the interest lies in loopy factor graphs. Whilst a general theory of loopy BP is lacking [84], it is known that equations (A.7) and (A.8) converge for general graphs, and that in the fixed point the error in approximation (A.9) depends on how far from a tree the graph actually is. In the next section, it is assumed that the BP procedure delivers a good approximation of the marginals for the Bayesian Compressed Sensing model a result which has been already argued for in $[8,7,74,17]$.

\section{Message Passing for Compressed Sensing}

Here we apply the formalism described above to the Bayesian Compressed Sensing problem. Since the posterior distribution over $\boldsymbol{x}$ is continuous, approximations to the messages will be introduced, in a procedure known as Approximate Message Passing [37, 100].

Given the Compressed Sensing problem

$$
P(\boldsymbol{x} \mid A, \boldsymbol{y})=\prod_{\mu=1}^{M} P\left(y^{\mu} \mid \mathbf{A}^{\mu} \cdot \boldsymbol{x}\right) \prod_{i=1}^{N} \phi\left(x_{i}\right),
$$

with sparse prior $\phi(x)=(1-\rho) \delta(x)+\rho g(x)$, the BP update equations (messages 
(A.7) and (A.8)) are written

$$
\begin{aligned}
& \nu_{\mu \rightarrow i}\left(x_{i}\right)=\frac{1}{Z_{\mu \rightarrow i}} \int \prod_{j \neq i} d x_{j} \nu_{j \rightarrow \mu}\left(x_{j}\right) P\left(y^{\mu} \mid u^{\mu}\right) \\
& \nu_{i \rightarrow \mu}\left(x_{i}\right)=\frac{1}{Z_{i \rightarrow \mu}} \phi\left(x_{i}\right) \prod_{\gamma \neq \mu} \nu_{\gamma \rightarrow i}\left(x_{i}\right),
\end{aligned}
$$

with $u^{\mu} \equiv \mathbf{A}^{\mu} \cdot \boldsymbol{x}$. Note that messages are not only functions of $x_{i}$ but also can be thought of as estimates of marginal distributions over altered graphical models where specific nodes have been removed. Therefore we can take messages (A.11) and define their averages and variances as

$$
\begin{aligned}
m_{i \rightarrow \mu} & \equiv \int d x_{i} x_{i} \nu_{i \rightarrow \mu}\left(x_{i}\right) \\
v_{i \rightarrow \mu} & \equiv \int d x_{i} x_{i}^{2} \nu_{i \rightarrow \mu}\left(x_{i}\right)-m_{i \rightarrow \mu}^{2}
\end{aligned}
$$

Our main objective is to find a Gaussian approximation to (A.11) — the somewhat lengthy calculation below serves this purpose. We begin by introducing the following identity:

$$
1 \equiv \int d u^{\mu} \delta\left(u^{\mu}-\sum_{i=1}^{N} A_{\mu i} x_{i}\right)=\int d u^{\mu} \frac{1}{2 \pi} \int d \hat{u}^{\mu} \exp \left[-i \hat{u}^{\mu}\left(u^{\mu}-\sum_{i=1}^{N} A_{\mu i} x_{i}\right)\right]
$$

and inserting it in equation (A.11), which yields

$$
\begin{aligned}
\nu_{\mu \rightarrow i}\left(x_{i}\right)=\frac{1}{2 \pi Z_{\mu \rightarrow i}} & \int d u^{\mu} P\left(y^{\mu} \mid u^{\mu}\right) \int d \hat{u}^{\mu} \times \\
& \times \exp \left[-i \hat{u}^{\mu}\left(u^{\mu}-A_{\mu i} x_{i}\right)\right] \prod_{j \neq i} \underbrace{\int d x_{j} \nu_{j \rightarrow \mu}\left(x_{j}\right) \exp \left(i \hat{u}^{\mu} A_{\mu j} x_{j}\right)}_{\equiv I_{j}} .
\end{aligned}
$$

But since $A_{\mu i} \sim O\left(N^{-1 / 2}\right)$, the exponential inside $I_{j}$ can be well approximated by its expansion up to terms of order $O\left(A_{\mu i}^{2}\right)$. The integral $I_{j}$ then becomes

$$
\begin{aligned}
I_{j} & \simeq \int d x_{j} \nu_{j \rightarrow \mu}\left(x_{j}\right)\left[1+i \hat{u}^{\mu} A_{\mu j} x_{j}+\frac{1}{2}\left(i \hat{u}^{\mu} A_{\mu j} x_{j}\right)^{2}\right] \\
& =\int d x_{j} \nu_{j \rightarrow \mu}\left(x_{j}\right)+\left(i \hat{u}^{\mu} A_{\mu j}\right) \int d x_{j} \nu_{j \rightarrow \mu}\left(x_{j}\right) x_{j}+\frac{1}{2}\left(i \hat{u}^{\mu} A_{\mu j}\right)^{2} \int d x_{j} \nu_{j \rightarrow \mu}\left(x_{j}\right) x_{j}^{2} \\
& =1+\left(i \hat{u}^{\mu} A_{\mu j}\right) m_{j \rightarrow \mu}+\frac{1}{2}\left(i \hat{u}^{\mu} A_{\mu j}\right)^{2}\left(v_{j \rightarrow \mu}+m_{j \rightarrow \mu}^{2}\right) .
\end{aligned}
$$

Now, note that an expansion of $\exp \left[\left(i \hat{u}^{\mu} A_{\mu j} m_{j \rightarrow \mu}\right)+\frac{1}{2}\left(i \hat{u}^{\mu} A_{\mu j}\right)^{2} v_{j \rightarrow \mu}\right]$ up to order 
$O\left(A_{\mu j}^{2}\right)$ also yields the same value:

$$
\begin{aligned}
\exp \left[\left(i \hat{u}^{\mu} A_{\mu j} m_{j \rightarrow \mu}\right)\right. & \left.+\frac{1}{2}\left(i \hat{u}^{\mu} A_{\mu j}\right)^{2} v_{j \rightarrow \mu}\right] \simeq \\
1 & +\left(i \hat{u}^{\mu} A_{\mu j}\right) m_{j \rightarrow \mu}+\frac{1}{2}\left(i \hat{u}^{\mu} A_{\mu j}\right)^{2} v_{j \rightarrow \mu}+\frac{1}{2}\left(i \hat{u}^{\mu} A_{\mu j}\right)^{2} m_{j \rightarrow \mu}^{2} .
\end{aligned}
$$

This means we can use this correspondence (which is valid with precision of order $\left.O\left(N^{-1}\right)\right)$ to replace the integral $I_{j}$ for the exponential in (A.18). The product $\prod_{j \neq i} I_{j}$ in (A.16) can then be readily calculated:

$$
\begin{aligned}
\prod_{j \neq i} I_{j} \simeq & \prod_{j \neq i} \exp \left[i \hat{u}^{\mu} A_{\mu j} m_{j \rightarrow \mu}+\frac{1}{2}\left(i \hat{u}^{\mu} A_{\mu j}\right)^{2} v_{j \rightarrow \mu}\right] \\
= & \left\{\prod_{j} \exp \left[i \hat{u}^{\mu} A_{\mu j} m_{j \rightarrow \mu}+\frac{1}{2}\left(i \hat{u}^{\mu} A_{\mu j}\right)^{2} v_{j \rightarrow \mu}\right]\right\} \times \\
& \times \exp \left[-i \hat{u}^{\mu} A_{\mu i} m_{i \rightarrow \mu}-\frac{1}{2}\left(i \hat{u}^{\mu} \Phi_{\mu i}\right)^{2} v_{i \rightarrow \mu}\right] \\
= & \exp \left[i \hat{u}^{\mu}\left(\Delta^{\mu}-A_{\mu i} m_{i \rightarrow \mu}\right)-\frac{\left(\hat{u}^{\mu}\right)^{2}}{2}\left(\chi^{\mu}-A_{\mu i}^{2} v_{i \rightarrow \mu}\right)\right],
\end{aligned}
$$

where the definitions $\Delta^{\mu}:=\sum_{i} A_{\mu i} m_{i \rightarrow \mu}$ and $\chi^{\mu}:=\sum_{i} A_{\mu i}^{2} v_{i \rightarrow \mu}$ were employed.

If we perform the Gaussian integral of $\hat{u}^{\mu}$ in (A.16) we shall obtain

$$
\begin{aligned}
& \nu_{\mu \rightarrow i}\left(x_{i}\right)=\frac{1}{Z_{\mu \rightarrow i} \sqrt{2 \pi\left(\chi^{\mu}-A_{\mu i}^{2} v_{i \rightarrow \mu}\right)}} \int d u^{\mu} P\left(y^{\mu} \mid u^{\mu}\right) \times \\
& \times \exp \left[-\frac{\left(u^{\mu}-\Delta^{\mu}-A_{\mu i}\left(x_{i}-m_{i \rightarrow \mu}\right)\right)^{2}}{2\left(\chi^{\mu}-A_{\mu i}^{2} v_{i \rightarrow \mu}\right)}\right] .
\end{aligned}
$$

But since $A_{\mu i}^{2} \sim O\left(N^{-1}\right)$ while $v_{i \rightarrow \mu} \sim O(1)$, we can omit the whole term $A_{\mu i}^{2} v_{i \rightarrow \mu}$ in the last equation. Also, $\chi^{\mu}=\sum_{i} A_{\mu i}^{2} v_{i \rightarrow \mu} \simeq(1 / N) \sum_{i} v_{i \rightarrow \mu}$. and all $\chi^{\mu}$ become equal to the average $\chi \equiv(1 / N) \sum_{i} v_{i}$ for $N \rightarrow \infty$, eliminating all dependencies on $\mu$. We can now expand the message (A.11) up to order $O\left(A_{\mu i}^{2}\right)$ as

$$
\begin{aligned}
\nu_{\mu \rightarrow i}\left(x_{i}\right) \propto & \int d u^{\mu} P\left(y^{\mu} \mid u^{\mu}\right) \exp \left[-\frac{\left(u^{\mu}-\Delta^{\mu}-A_{\mu i}\left(x_{i}-m_{i \rightarrow \mu}\right)\right)^{2}}{2 \chi}\right] \\
= & \int d u^{\mu} P\left(y^{\mu} \mid u^{\mu}\right) \exp \left[-\frac{\left(u^{\mu}-\Delta^{\mu}\right)^{2}}{2 \chi}\right] \\
& \times \exp \frac{1}{2 \chi}\left[\left(A_{\mu i}\left(x_{i}-m_{i \rightarrow \mu}\right)\right)^{2}-2\left(u^{\mu}-\Delta^{\mu}\right)\left(A_{\mu i}\left(x_{i}-m_{i \rightarrow \mu}\right)\right)\right] \\
\simeq & c_{0}+c_{1} A_{\mu i}\left(x_{i}-m_{i \rightarrow \mu}\right)+\frac{1}{2} c_{2} A_{\mu i}^{2}\left(x_{i}-m_{i \rightarrow \mu}\right)^{2}
\end{aligned}
$$


where

$$
\begin{aligned}
& c_{0} \equiv \int d u_{\mu} P\left(y^{\mu} \mid u^{\mu}\right) \exp \left[-\frac{\left(u^{\mu}-\Delta^{\mu}\right)^{2}}{2 \chi}\right], \\
& c_{1} \equiv \frac{\partial c_{0}}{\partial \Delta^{\mu}}=\int d u_{\mu} P\left(y^{\mu} \mid u^{\mu}\right)\left(\frac{u_{\mu}-\Delta_{\mu}}{\chi}\right) \exp \left[-\frac{\left(u^{\mu}-\Delta^{\mu}\right)^{2}}{2 \chi}\right], \\
& c_{2} \equiv \frac{\partial^{2} c_{0}}{\partial\left(\Delta^{\mu}\right)^{2}}=\int d u_{\mu} P\left(y^{\mu} \mid u^{\mu}\right)\left(\left(\frac{u_{\mu}-\Delta_{\mu}}{\chi}\right)^{2}-\frac{1}{\chi}\right) \exp \left[-\frac{\left(u^{\mu}-\Delta^{\mu}\right)^{2}}{2 \chi}\right] .
\end{aligned}
$$

If we write $\nu_{\mu \rightarrow i}\left(x_{i}\right) \simeq c_{0}\left[1+\left(c_{1} / c_{0}\right) A_{\mu i}\left(x_{i}-m_{i \rightarrow \mu}\right)+\frac{1}{2}\left(c_{2} / c_{0}\right) A_{\mu i}^{2}\left(x_{i}-m_{i \rightarrow \mu}\right)^{2}\right]$, it can be even further approximated by

$$
\begin{aligned}
\nu_{\mu \rightarrow i}\left(x_{i}\right)= & \exp \left\{\ln c_{0}+\ln \left[1+\frac{c_{1}}{c_{0}} A_{\mu i}\left(x_{i}-m_{i \rightarrow \mu}\right)+\frac{1}{2} \frac{c_{2}}{c_{0}} A_{\mu i}^{2}\left(x_{i}-m_{i \rightarrow \mu}\right)^{2}\right]\right\} \\
\simeq & \exp \left[\ln c_{0}+\frac{c_{1}}{c_{0}} A_{\mu i}\left(x_{i}-m_{i \rightarrow \mu}\right)+\frac{c_{0} c_{2}-c_{1}^{2}}{2 c_{0}^{2}} A_{\mu i}^{2}\left(x_{i}-m_{i \rightarrow \mu}\right)^{2}\right] \\
= & \exp \left[\ln c_{0}-\frac{c_{1}}{c_{0}} A_{\mu i} m_{i \rightarrow \mu}+\frac{c_{0} c_{2}-c_{1}^{2}}{2 c_{0}^{2}} A_{\mu i}^{2} m_{i \rightarrow \mu}^{2}\right] \\
& \times \exp \left[\frac{c_{1}}{c_{0}} A_{\mu i} x_{i}+\frac{c_{0} c_{2}-c_{1}^{2}}{2 c_{0}^{2}} A_{\mu i}^{2} x_{i}^{2}-\frac{c_{0} c_{2}-c_{1}^{2}}{c_{0}^{2}} A_{\mu i}^{2} x_{i} m_{i \rightarrow \mu}\right] .
\end{aligned}
$$

Note that all terms dependent on $x_{i}$ are in a Gaussian form, so that we have arrived at the intended Gaussian approximation for the messages:

$$
\nu_{\mu \rightarrow i}\left(x_{i}\right) \propto \exp \left[-\frac{F_{\mu \rightarrow i}}{2} x_{i}^{2}+G_{\mu \rightarrow i} x_{i}\right],
$$

where

$$
\begin{aligned}
F_{\mu \rightarrow i} & \equiv \frac{c_{1}^{2}-c_{0} c_{2}}{c_{0}^{2}} A_{\mu i}^{2}, \\
G_{\mu \rightarrow i} & \equiv \frac{c_{1}}{c_{0}} A_{\mu i}+\frac{c_{1}^{2}-c_{0} c_{2}}{c_{0}^{2}} A_{\mu i}^{2} m_{i \rightarrow \mu} .
\end{aligned}
$$

With these, the node-to-factor messages are obtained by substitution of (A.30) in (A.12):

$$
\nu_{i \rightarrow \mu}\left(x_{i}\right) \simeq \frac{1}{\tilde{Z}_{i \rightarrow \mu}}\left[(1-\rho) \delta\left(x_{i}\right)+\rho g\left(x_{i}\right)\right] \mathrm{e}^{-\left(x_{i}^{2} / 2\right) \sum_{\gamma \neq \mu} F_{\gamma \rightarrow i}+x_{i} \sum_{\gamma \neq \mu} G_{\gamma \rightarrow i}} .
$$

The closed iterative set of equations (A.13), (A.14), (A.31), (A.32) and (A.33) already correspond to a message passing algorithm for Compressed Sensing. The updates of these $2 M N$ messages have a computational cost of $O\left(M^{2} \times N+M \times N^{2}\right)$. For large $N$, it is possible to approximate these messages and write them as $M+N$ 
messages with much cheaper computational cost: $O(M N)$. The next section will be concerned with the introduction of these approximations.

\section{Approximate Message Passing}

All that is left to arrive at a closed-form equation for the means (A.13) and variances (A.14) is a explicit expression for $g(x)$ in (A.33). When the prior is a Gauss-Bernoulli distribution, i.e. $g(x)=\left(1 / \sqrt{2 \pi \sigma^{2}}\right) \exp \left[-x^{2} / 2 \sigma^{2}\right]$, the means and variances of (A.33) can be directly calculated. They are then given by the following expressions:

$$
\begin{aligned}
m_{i \rightarrow \mu} & =\Psi_{m}\left(\sum_{\gamma \neq \mu} F_{\gamma \rightarrow i}, \sum_{\gamma \neq \mu} G_{\gamma \rightarrow i}\right), \\
v_{i \rightarrow \mu} & =\Psi_{v}\left(\sum_{\gamma \neq \mu} F_{\gamma \rightarrow i}, \sum_{\gamma \neq \mu} G_{\gamma \rightarrow i}\right),
\end{aligned}
$$

where

$$
\Psi_{m}(s, t):=\frac{\rho t}{\sigma\left(1 / \sigma^{2}+s\right)^{3 / 2}}\left[(1-\rho) e^{t^{2} /\left[2\left(1 / \sigma^{2}+s\right)\right]}+\frac{\rho}{\sigma\left(1 / \sigma^{2}+s\right)^{1 / 2}}\right]^{-1}
$$

and

$$
\begin{aligned}
\Psi_{v}(s, t):= & \frac{\rho}{\sigma\left(1 / \sigma^{2}+s\right)^{3 / 2}}\left(1+\frac{t^{2}}{1 / \sigma^{2}+s}\right) \times \\
& \times\left[(1-\rho) e^{t^{2} /\left[2\left(1 / \sigma^{2}+s\right)\right]}+\frac{\rho}{\sigma\left(1 / \sigma^{2}+s\right)^{1 / 2}}\right]^{-1}-\Psi_{m}^{2}(s, t) .
\end{aligned}
$$

Equations (A.13) and (A.14) are then, according to (A.9),

$$
\begin{aligned}
m_{i} & =\Psi_{m}\left(\sum_{\gamma} F_{\gamma \rightarrow i}, \sum_{\gamma} G_{\gamma \rightarrow i}\right), \\
v_{i} & =\Psi_{v}\left(\sum_{\gamma} F_{\gamma \rightarrow i}, \sum_{\gamma} G_{\gamma \rightarrow i}\right) .
\end{aligned}
$$

Let us introduce a specific format for the likelihood $P\left(y^{\mu} \mid u^{\mu}\right)$ as well and consider the standard noisy Compressed Sensing scenario

$$
P\left(y^{\mu} \mid u^{\mu}\right)=\frac{1}{\sqrt{2 \pi \sigma_{n}^{2}}} \exp \left[-\frac{1}{2 \sigma_{n}^{2}}\left(y^{\mu}-u^{\mu}\right)^{2}\right] .
$$

This specification allows the calculation of the $c_{j}$ constants (A.24), (A.25) and 
(A.26), so that

$$
\begin{gathered}
F_{\mu \rightarrow i}=\frac{A_{\mu i}^{2}}{\Delta^{\mu}+\sum_{j \neq i} A_{\mu j}^{2} v_{j \rightarrow \mu}}=\frac{A_{\mu i}^{2}}{\Delta^{\mu}+\gamma^{\mu}-A_{\mu i}^{2} v_{i \rightarrow \mu}}, \\
G_{\mu \rightarrow i}=\frac{A_{\mu i}\left(y^{\mu}-\sum_{j \neq i} A_{\mu j} m_{j \rightarrow \mu}\right)}{\Delta^{\mu}+\sum_{j \neq i} A_{\mu j}^{2} v_{j \rightarrow \mu}}=\frac{A_{\mu i}\left(y^{\mu}-\omega^{\mu}+A_{\mu i} m_{i \rightarrow \mu}\right)}{\Delta^{\mu}+\gamma^{\mu}-A_{\mu i}^{2} v_{i \rightarrow \mu}},
\end{gathered}
$$

where we defined the summations $\omega^{\mu}:=\sum_{j} A_{\mu j} m_{j \rightarrow \mu}$ and $\gamma^{\mu}:=\sum_{j} A_{\mu j}^{2} v_{j \rightarrow \mu}$.

The Approximate Message Passing algorithm is obtained by taking the limit of $N \rightarrow \infty$, simplifying even more the formulas above. With the reminder that $A_{\mu i} \sim O(1 / N)$, we can define the macroscopic variables

$$
U_{i}:=\sum_{\mu} F_{\mu \rightarrow i} \simeq \sum_{\mu} \frac{A_{\mu i}^{2}}{\Delta^{\mu}+\gamma^{\mu}}
$$

and

$$
V_{i}:=\sum_{\mu} G_{\mu \rightarrow i} \simeq \sum_{\mu} A_{\mu i} \frac{y^{\mu}-\omega^{\mu}}{\Delta^{\mu}+\gamma^{\mu}}+\Psi_{a}\left(U_{i}, V_{i}\right) \sum_{\mu} A_{\mu i}^{2} \frac{1}{\Delta^{\mu}+\gamma^{\mu}} .
$$

Parameters $\omega^{\mu}$ and $\gamma^{\mu}$ by themselves can also be simplified in this regime. With

$$
\begin{aligned}
m_{i \rightarrow \mu} & =\Psi_{a}\left(U_{i}-F_{\mu \rightarrow i}, V_{i}-G_{\mu \rightarrow i}\right) \\
& =\Psi_{a}\left(U_{i}, V_{i}\right)-\left.F_{\mu \rightarrow i} \frac{\partial \Psi_{a}\left(u, V_{i}\right)}{\partial u}\right|_{U_{i}}-\left.G_{\mu \rightarrow i} \frac{\partial \Psi_{a}\left(U_{i}, v\right)}{\partial v}\right|_{V_{i}},
\end{aligned}
$$

they become

$$
\omega^{\mu}=\sum_{i} A_{\mu i} m_{i \rightarrow \mu}=\sum_{i} A_{\mu i} \Psi_{a}\left(U_{i}, V_{i}\right)-\left.\frac{y^{\mu}-\omega^{\mu}}{\Delta^{\mu}+\gamma^{\mu}} \sum_{i} A_{\mu i}^{2} \frac{\partial \Psi_{a}\left(U_{i}, v\right)}{\partial v}\right|_{V_{i}}
$$

and

$\gamma^{\mu}=\sum_{i} A_{\mu i}^{2} v_{i \rightarrow \mu}=\sum_{i} A_{\mu i}^{2} \Psi_{v}\left(U_{i}, V_{i}\right)-\left.\frac{y^{\mu}-\omega^{\mu}}{\Delta^{\mu}+\gamma^{\mu}} \sum_{i} A_{\mu i}^{3} \frac{\partial \Psi_{v}\left(U_{i}, v\right)}{\partial v}\right|_{V_{i}} \simeq \sum_{i} A_{\mu i}^{2} \Psi_{v}\left(U_{i}, V_{i}\right)$

The system of iterative equations (A.42), (A.43), (A.47) and (A.46) is what is known as the Approximate Message Passing algorithm. Expressions (A.38) and (A.39) tell us that, at any iteration, an estimate of the original signal might be obtained from $m_{i}=\Psi_{m}\left(U_{i}, V_{i}\right)$ and $v_{i}=\Psi_{v}\left(U_{i}, V_{i}\right)$, effectively closing the system. Chapter 2 (and particularly Figure 2.1) presents results from simulations of this scheme. In Figure 2.3 it is possible to see the convergence between results obtained with the AMP algorithm and the replica prediction for infinite systems. 



\section{CHAPTER B}

\section{1-Bit Compressed Sensing}

Alternative to the Standard Compressed Sensing scenario, in 1-bit Compressed Sensing all measurements $y^{\mu}$ go through a quantizer in the form of a comparator to zero. In other words, all measurement amplitudes are discarded and the only information left is their signals. They can be written

$$
\boldsymbol{y}=\operatorname{sgn}\left(\underline{A} \boldsymbol{x}_{0}\right),
$$

where $\operatorname{sgn}(u)=u /|u|$ for $x \neq 0$ (and $\operatorname{sgn}(0)=0$ ) is a function applied to each element $u_{0}^{\mu} \equiv \mathbf{A}^{\mu} \cdot \boldsymbol{x}_{0}$ independently. In presence of additive noise, we consider

$$
\boldsymbol{y}=\operatorname{sgn}\left(\underline{A} \boldsymbol{x}_{0}+\boldsymbol{\xi}\right)
$$

with $\boldsymbol{\xi}=\left(\xi^{\mu}\right)$, and $\xi^{\mu} \sim \mathcal{N}\left(0, \sigma_{n}^{2}\right)$. A measurement process like this results in obvious loss of information meaning one cannot expect to recover the signal $\boldsymbol{x}_{0}$ exactly, even for $M>N$. This means that, like in the Online CS setting, the range of interest for the parameter $\alpha$ changes. In traditional Compressed Sensing problems, it is of common interest to have $\alpha<1$ otherwise other solution methods could be used. In 1-bit CS the interest lies in $\alpha \gg 1$. The connection with Online CS is then made clear — both rely on the theoretical framework of Compressed Sensing while at the same time giving up on the main goal of reducing measurement numbers in order to achieve other objectives. Nevertheless, the case is of interest since these comparators to zero are fast and inexpensive hardware devices which do not saturate [14]. In situations where perfect recovery is not required and the cost of data storage (quantified by the total number of bits needed to store the measurements) is high, discarding the amplitude information can be highly advantageous, since it greatly reduces the amount of data needed to store and/or transmit the results. In the 1-bit CS scenario, measurements are considered to be extremely cheap in stark contrast 
to storage, which is considered expensive.

Following the spirit of traditional CS, Boufounos and Baraniuk [14] introduced the idea of 1-bit CS with a proposed signal reconstruction technique of the same $\ell_{1}$-minimization form that was already proven successful in the field. But whereas in Standard CS it was desirable that any solution $\boldsymbol{x}$ obeyed $\boldsymbol{y}=\underline{A} \boldsymbol{x}$ (at least close enough), this is too hard of a constraint in a formalism like this. Instead, this requirement is relaxed to the notion of consistency: since it is impossible to recover the signal exactly, it is at least desirable that the reconstructed signal $\boldsymbol{x}$ gives rise to the same measurements $\boldsymbol{y}$ in case it was to be measured in the same way $\boldsymbol{x}_{0}$ was. In other words, any successful reconstruction $\boldsymbol{x}$ must obey $\left(\operatorname{sgn}\left(\mathbf{A}^{\mu} \cdot \boldsymbol{x}_{0}\right)\right)=\left(\operatorname{sgn}\left(\mathbf{A}^{\mu} \cdot \boldsymbol{x}\right)\right)$ for all $\mu=1, \ldots M$. This expression can also be written

$$
\left(\operatorname{sgn}\left(\underline{A} \boldsymbol{x}_{0}\right)\right)^{\mu}(\operatorname{sgn}(\underline{A} \boldsymbol{x}))^{\mu} \geq 0 .
$$

There is one caveat, though - by the very nature of the measurements, if any solution $\boldsymbol{x}$ is consistent then $k \boldsymbol{x}$ is also consistent for every $0<k \leq 1$. This implies that a minimization algorithm would then drive the solution to $\boldsymbol{x}=0$ always, since $\|k \boldsymbol{x}\|_{1}=k\|\boldsymbol{x}\|_{1}<\|\boldsymbol{x}\|_{1}$. For this reason it is desirable to constrain the solution to the $\ell_{2}$ unity sphere, eliminating this ambiguity while at the same time reducing the optimization search space. The $\left(P_{1}\right)$ problem then becomes, in the 1-bit CS formalism:

$$
\hat{\boldsymbol{x}}=\underset{\boldsymbol{x}}{\arg \min }\|\boldsymbol{x}\|_{1} \text { s.t. } \underline{Y} \underline{A} \boldsymbol{x} \geq 0 \text { and }\|\boldsymbol{x}\|_{2}=1,
$$

where $\underline{Y}:=\operatorname{diag}\left(y^{1}, \ldots, y^{M}\right)$ is a $M \times M$ matrix with the elements $y^{1}, \ldots, y^{M}$ in its diagonal.

A Bayesian approach can be formulated for 1-bit CS as well and it is guaranteed to achieve optimal performance [137]. The posterior distribution for the noiseless inference problem becomes

$$
P(\boldsymbol{x} \mid \underline{A}, \boldsymbol{y}) \propto \prod_{i=1}^{N}\left[(1-\rho) \delta\left(x_{i}\right)+\rho g\left(x_{i}\right)\right] \prod_{\mu=1}^{M} \Theta\left[y^{\mu} \mathbf{A}^{\mu} \cdot \boldsymbol{x}\right]
$$

paralleling equation (2.7). Here, $\Theta(x)=1$ for $x \geq 0$ and $\Theta(x)=0$ otherwise. A replica analysis of (B.5) can also be made - in fact, under the replica symmetric conditions, the calculation is exactly the same as the one shown in Chapter 2, up to the free energy expression (2.16). When perfect knowledge of the signal generating distribution is available, the introduction of the noiseless 1-bit CS measurement 
model $P(y \mid u) \propto \Theta(y u)$ leads to the following saddle-point equations:

$$
\left\{\begin{array}{l}
q=\int \mathcal{D} t \frac{\left(\int d x x e^{-\frac{\hat{q}}{2} x^{2}+\sqrt{\hat{q} t} x} \phi(x)\right)^{2}}{\int d x e^{-\frac{\hat{q}}{2} x^{2}+\sqrt{\hat{q} t} x} \phi(x)} \\
\hat{q}=\frac{\alpha}{\pi \sqrt{2 \pi(\rho-q)}} \int d t \frac{\exp \left[-\frac{\rho+q}{2(\rho-q)} t^{2}\right]}{H\left(\sqrt{\frac{q}{\rho-q}} t\right)} .
\end{array}\right.
$$

Solutions to these equations (as well as for the noisy case, which is not calculated here), are shown in Section 3.3 to serve as a benchmark for the Bayesian Online Compressed Sensing algorithm performance. 



\section{BIBLIOGRAPHY}

[1] Akaike, H. Likelihood and the bayes procedure. Trabajos de estadística y de investigación operativa 31, 1 (1980), 143-166.

[2] AkÇAkaya, M., And Tarokh, V. Shannon-theoretic limits on noisy compressive sampling. IEEE Transactions on Information Theory 56, 1 (2010), 492-504.

[3] Amari, S.-I., And Nagaoka, H. Methods of Information Geometry. American Mathematical Soc., 2007.

[4] Barber, D. Bayesian Reasoning and Machine Learning. Cambridge University Press, 2012.

[5] Baron, D., Duarte, M. F., Wakin, M. B., Sarvotham, S., and BaraNIUK, R. G. Distributed compressive sensing. arXiv:0901.3403, 2009.

[6] Baron, D., Sarvotham, S., and Baraniuk, R. G. Bayesian compressive sensing via belief propagation. IEEE TRANSACTIONS ON SIGNAL PROCESSING 58-1 (2010), 269.

[7] Bayati, M., Lelarge, M., Montanari, A., et Al. Universality in polytope phase transitions and message passing algorithms. The Annals of Applied Probability 25, 2 (2015), 753-822.

[8] Bayati, M., And Montanari, A. The dynamics of message passing on dense graphs, with applications to compressed sensing. IEEE TRANSACTIONS ON INFORMATION THEORY 57-2 (2011), 764.

[9] Bayes, M., And Price, M. An essay towards solving a problem in the doctrine of chances. by the late rev. mr. bayes, frs communicated by $\mathrm{mr}$. price, in a letter to john canton, amfrs. Philosophical Transactions (16831775) (1763), 370-418.

[10] Berger, J. O. Statistical decision theory and Bayesian analysis. Springer Science \& Business Media, 2013.

[11] Berger, M. Geometry I. Springer Science \& Business Media, 2009.

[12] Bernoulli, J. Ars conjectandi. Impensis Thurnisiorum, fratrum, 1713. 
[13] Biehl, M., And Riegler, P. On-line learning with a perceptron. EPL (Europhysics Letters) 28, 7 (1994), 525.

[14] Boufounos, P. T., And Baraniuk, R. G. 1-bit compressive sensing. In Information Sciences and Systems, 2008. CISS 2008. 42nd Annual Conference on (2008), IEEE, pp. 16-21.

[15] Broderick, T., Boyd, N., Wibisono, A., Wilson, A. C., And JorDAN, M. I. Streaming variational bayes. In Advances in Neural Information Processing Systems (2013), pp. 1727-1735.

[16] Bulaevskit, L., Panyukov, S., and Sadovskit, M. Inhomogeneous superconductivity in disordered metals. Zh. Eksp. Teor. Fiz. 92, 2 (1987), 672688.

[17] Caltagirone, F., Zdeborová, L., and Krzakala, F. On convergence of approximate message passing. In Information Theory (ISIT), 2014 IEEE International Symposium on (2014), IEEE, pp. 1812-1816.

[18] Candès, E., And Romberg, J. Sparsity and incoherence in compressive sampling. Inverse problems 23, 3 (2007), 969.

[19] CAndès, E., AND TAO, T. Near optimal signal recovery from random projections: Universal encoding strategies? IEEE TRANSACTIONS ON INFORMATION THEORY 52-12 (2006), 5406-5425.

[20] CAndès, E. J. The restricted isometry property and its implications for compressed sensing. Comptes Rendus Mathematique 346, 9 (2008), 589-592.

[21] Candès, E. J., Romberg, J., and TaO, T. Robust uncertainty principles: Exact signal reconstruction from highly incomplete frequency information. IEEE TRANSACTIONS ON INFORMATION THEORY 52-2 (2006), 489.

[22] Candès, E. J., And TAO, T. Decoding by linear programming. IEEE transactions on information theory 51, 12 (2005), 4203-4215.

[23] Candès, E. J., And Wakin, M. B. An introduction to compressive sampling. IEEE SIGNAL PROCESSING MAGAZINE (2008), 21.

[24] Caticha, N., Calsaverini, R., And Vicente, R. Phase transition from egalitarian to hierarchical societies driven by competition between cognitive and social constraints. arXiv preprint arXiv:1608.03637 (2016).

[25] Chen, S. S., Donoho, D. L., and Saunders, M. A. Atomic decomposition by basis pursuit. SIAM review 43, 1 (2001), 129-159.

[26] Claerbout, J. F., And Muir, F. Robust modeling with erratic data. Geophysics 38, 5 (1973), 826-844.

[27] Cover, T. M., And Thomas, J. A. Elements of Information Theory. Wiley, 2006. 
[28] Cox, R. T. Probability, frequency and reasonable expectation. American journal of physics 14, 1 (1946), 1-13.

[29] Cox, R. T. Algebra of Probable Inference. JHU Press, 1961.

[30] Cukier, K. Data, data everywhere: A special report on managing information. Economist Newspaper, 2010.

[31] Davenport, M. A., Laska, J. N., Boufounos, P. T., And Baraniuk, R. G. A simple proof that random matrices are democratic. arXiv preprint arXiv:0911.0736 (2009).

[32] De Oliveira, E. A., And Caticha, N. Inference from aging information. Neural Networks, IEEE Transactions on 21, 6 (2010), 1015-1020.

[33] Donoho, D. L. Compressed sensing. IEEE Transactions on information theory 52, 4 (2006), 1289-1306.

[34] Donoho, D. L. High-dimensional centrally symmetric polytopes with neighborliness proportional to dimension. Discrete Comput. Geom. 35 (2006), 617652.

[35] Donoho, D. L., And Elad, M. Optimally sparse representation in general (nonorthogonal) dictionaries via $\ell_{1}$ minimization. Proceedings of the National Academy of Sciences 100, 5 (2003), 2197-2202.

[36] Donoho, D. L., Maleki, A., And Montanari, A. Message passing algorithms for compressed sensing: I. motivation and construction. In Information Theory Workshop (ITW), 2010 IEEE (2010), IEEE, pp. 1-5.

[37] Donoho, D. L., Maleki, A., And Montanari, A. How to design message passing algorithms for compressed sensing. preprint (2011).

[38] Donoho, D. L., Malekib, A., And Montanari, A. Message-passing algorithms for compressed sensing. PNAS 106-45 (2009), 18914.

[39] Donoho, D. L., And Tanner, J. Observed universality of phase transitions in high-dimensional geometry, with implications for modern data analysis and signal processing. Philosophical Transactions of the Royal Society A 367-1906 (2009), 4273-4293.

[40] Drémeau, A., Herzet, C., and Daudet, L. Boltzmann machine and mean-field approximation for structured sparse decompositions. IEEE Transactions on Signal Processing 60, 7 (2012), 3425-3438.

[41] Duarte, M., And Eldar, Y. Structured compressed sensing: From theory to applications. IEEE SIGNAL PROCESSING MAGAZINE 59, 9 (2011), 4053-4085.

[42] Duarte, M. F., Davenport, M. A., Takbar, D., Laska, J. N., Sun, T., Kelly, K. F., And Baraniuk, R. G. Single-pixel imaging via compressive sampling. IEEE signal processing magazine 25, 2 (2008), 83-91. 
[43] Duarte, M. F., Sarvotham, S., Baron, D., Wakin, M. B., and BaraNIUK, R. G. Distributed compressed sensing of jointly sparse signals. In In Asilomar Conf. Signals, Sys., Comput (2005), pp. 1537-1541.

[44] Duarte, M. F., Wakin, M. B., and Baraniuk, R. G. Fast reconstruction of piecewise smooth signals from incoherent projections. Proc. SPARS (2005).

[45] Edwards, S. This week's citation classic. Current Contents 37 (1985), 20.

[46] Edwards, S. F., And Anderson, P. W. Theory of spin glasses. Journal of Physics F: Metal Physics 5, 5 (1975), 965.

[47] Efron, B., Hastie, T., Johnstone, I., Tibshirani, R., Et Al. Least angle regression. The Annals of statistics 32, 2 (2004), 407-499.

[48] Elad, M. Sparse and Redundant Representations: From Theory to Applications in Signal and Image Processing. Springer, 2010.

[49] Elad, M. Sparse and redundant representation modelingâĂ ̌̌what next? IEEE Signal Processing Letters 19, 12 (2012), 922-928.

[50] Elad, M., And Bruckstein, A. M. A generalized uncertainty principle and sparse representation in pairs of bases. IEEE Transactions on Information Theory 48, 9 (2002), 2558-2567.

[51] Eldar, Y. C., And Kutyniok, G. Compressed Sensing. Cambridge, 2012.

[52] Engel, A., And den Broeck, V. Statistical Mechanics of Learning. Cambridge University Press, 2001.

[53] Fancher, C. M., Han, Z., Levin, I., Page, K., Reich, B. J., Smith, R. C., Wilson, A. G., And Jones, J. L. Use of bayesian inference in crystallographic structure refinement via full diffraction profile analysis. Scientific reports 6 (2016), 31625 .

[54] Figueiredo, M. A. Adaptive sparseness for supervised learning. IEEE transactions on pattern analysis and machine intelligence 25, 9 (2003), 11501159.

[55] Fletcher, A. K., Rangan, S., and Goyal, V. K. Necessary and sufficient conditions for sparsity pattern recovery. IEEE Transactions on Information Theory 55, 12 (2009), 5758-5772.

[56] Fowler, J. E., Mun, S., Tramel, E. W., ET AL. Block-based compressed sensing of images and video. Foundations and Trends® in Signal Processing 4, 4 (2012), 297-416.

[57] Fu, W. J. Penalized regressions: the bridge versus the lasso. Journal of computational and graphical statistics 7, 3 (1998), 397-416.

[58] Ganguli, S., And Sompolinsky, H. Statistical mechanics of compressed sensing. PHYSICAL REVIEW LETTERS 104 (2010), 188701. 
[59] Gribonval, R., and Nielsen, M. Sparse representations in unions of bases. IEEE transactions on Information theory 49, 12 (2003), 3320-3325.

[60] Guo, D., And Verdú, S. Randomly spread cdma: Asymptotics via statistical physics. IEEE Transactions on Information Theory 51, 6 (2005), 19832010 .

[61] Hans, C. Bayesian lasso regression. Biometrika 96, 4 (2009), 835-845.

[62] Hey, T., Tansley, S., And Tolle, K., Eds. The Fourth Padadigm: DataIntensive Scientific Discovery. Microsoft Research, 2009.

[63] Hey, T., And Trefethen, A. E. Cyberinfrastructure for e-science. Science 308 (2005), 817.

[64] Holtz, O. Compressive sensing: a paradigm shift in signal processing. arXiv:0812.3137, 2008.

[65] Huelsenbeck, J. P., And Ronquist, F. Mrbayes: Bayesian inference of phylogenetic trees. Bioinformatics 17, 8 (2001), 754-755.

[66] Huson, D. H., Rupp, R., and Scornavacca, C. Phylogenetic networks: concepts, algorithms and applications. Cambridge University Press, 2010.

[67] IBA, Y. The nishimori line and bayesian statistics. Journal of Physics A: Mathematical and General 32, 21 (1999), 3875.

[68] Jaynes, E. T. Probability theory: the logic of science. Cambridge university press, 2003.

[69] Kabashima, Y. Perfect loss of generalization due to noise in $\mathrm{k}=2$ parity machines. J. Phys. A: Math. Gen. 27 (1994), 1917.

[70] Kabashima, Y., Wadayama, T., and Tanaka, T. A typical reconstruction limit for compressed sensing based on lp-norm minimization. Journal of Statistical Mechanics: Theory and Experiment (2009), L09003.

[71] Kamei, H., Takayasu, H., Kabashima, Y., and Takayasu, M. Bankruptcy prediction with interfirm network structure. In Proceedings of the Asia-Pacific Econophysics Conference 2016âĂ ૅ̌Big Data Analysis and Modeling toward Super Smart Societyâ̆ $\breve{T}(A P E C-S S S 2016)$ (2017), p. 011013.

[72] Kinouchi, O., And Caticha, N. Optimal generalization in perceptions. J. Phys. A: Math. Gen. 25 (1992), 6243.

[73] Koop, G., Korobilis, D., ET AL. Bayesian multivariate time series methods for empirical macroeconomics. Foundations and Trends® in Econometrics 3, 4 (2010), 267-358. 
[74] Krzakala, F., Mézard, M., Sausset, F., Sun, Y., and Zdeborova, L. Probabilistic reconstruction in compressed sensing: algorithms, phase diagrams, and threshold achieving matrices. Journal of Statistical Mechanics: Theory and Experiment (2012), P08009.

[75] Krzakala, F., Mézard, M., Sausset, F., and Zdeborova, L. Statistical-physics-based reconstruction in compressed sensing. PHYSICAL REVIEW X 2 (2012), 021005.

[76] LA, C., AND Do, M. Signal reconstruction using sparse tree representations. SPIE Wavelets XI 5914 (2005), 59140W.

[77] Laplace, P. S. Memoir on the probability of the causes of events. Statistical Science 1, 3 (1986), 364-378.

[78] Mackay, D. J. Bayesian interpolation. Neural computation 4, 3 (1992), $415-447$.

[79] Majumdar, A., Ward, R. K., And Aboulnasr, T. Compressed sensing based real-time dynamic mri reconstruction. IEEE Transactions on Medical Imaging 31, 12 (2012), 2253-2266.

[80] Malioutov, D. M., Sanghavi, S. R., And Willsky, A. S. Sequential compressed sensing. IEEE Journal of Selected Topics in Signal Processing 4 , 2 (2010), 435-444.

[81] Manoel, A., Krzakala, F., Tramel, E. W., And Zdeborová, L. Streaming bayesian inference: theoretical limits and mini-batch approximate message-passing. arXiv preprint arXiv:1706.00705 (2017).

[82] Marquis De Laplace, P. S. Théorie analytique des probabilités. Mme Ve Courcier, 1812.

[83] McGrayne, S. B. The theory that would not die: how Bayes' rule cracked the enigma code, hunted down Russian submarines, $\mathscr{6}$ emerged triumphant from two centuries of controversy. Yale University Press, 2011.

[84] Mézard, M., and Montanari, A. Information, Physics and Computation. Oxford University Press, 2009.

[85] Mézard, M., Parisi, G., And Virasoro, M. Spin glass theory and beyond: An Introduction to the Replica Method and Its Applications, vol. 9. World Scientific Publishing Co Inc, 1987.

[86] Mitliagkas, I., Caramanis, C., And Jain, P. Memory limited, streaming pca. In Advances in Neural Information Processing Systems (2013), pp. 28862894.

[87] Montanari, A. Graphical models concepts in compressed sensing. Compressed Sensing: Theory and Applications (2012), 394-438. 
[88] Muller, R. R. Channel capacity and minimum probability of error in large dual antenna array systems with binary modulation. IEEE Transactions on Signal Processing 51, 11 (2003), 2821-2828.

[89] Muthukrishnan, S. Data Streams: Algorithms and Applications, Foundations and Trends in Theoretical Computer Science. Now Publishers, 2005.

[90] Natarajan, B. K. Sparse approximate solutions to linear systems. SIAM journal on computing 24, 2 (1995), 227-234.

[91] NeAL, R. M. Bayesian learning for neural networks, vol. 118. Springer Science \& Business Media, 2012.

[92] NG, A. Y. Feature selection, 11 vs. 12 regularization, and rotational invariance. In Proceedings of the twenty-first international conference on Machine learning (2004), ACM, p. 78.

[93] Nishimori, H. Statistical Physics of Spin Glasses and Information Processing. Oxford University Press, 2001.

[94] Nyquist, H. Certain topics in telegraph transmission theory. Trans. AIEE 47 (1928), 617-644.

[95] Opper, M. On-line versus off-line learning from random examples: General results. Phys. Rev. Lett. 77 (1996), 4671.

[96] Opper, M., And Winther, O. A bayesian approach to on-line learning. On-line Learning in Neural Networks, ed. D. Saad (1998), 363-378.

[97] Park, T., And Casella, G. The bayesian lasso. Journal of the American Statistical Association 103, 482 (2008), 681-686.

[98] PrugovečKi, E. Quantum mechanics in Hilbert space. Academic Press, 1981.

[99] Ramirez, C., Kreinovich, V., And Argaez, M. Why $\ell 1$ is a good approximation to $\ell$ : A geometric explanation. Journal of Uncertain Systems (2013).

[100] Rangan, S. Generalized approximate message passing for estimation with random linear mixing. Information Theory Proceedings (ISIT), 2011 IEEE International Symposium on (2011).

[101] Rangan, S., Fletcher, A. K., and Goyal, V. K. Asymptotic analysis of map estimation via the replica method and applications to compressed sensing. IEEE TRANSACTIONS ON INFORMATION THEORY 58-3 (2012), 1902.

[102] Reeves, G., And Gastpar, M. Compressed sensing phase transitions: Rigorous bounds versus replica predictions. 46th Annual Conference on Information Sciences and Systems (CISS) (2012), 1-6. 
[103] Richard, M. D., and Lippmann, R. P. Neural network classifiers estimate bayesian a posteriori probabilities. Neural computation 3, 4 (1991), 461-483.

[104] Ronquist, F., And Huelsenbeck, J. P. Mrbayes 3: Bayesian phylogenetic inference under mixed models. Bioinformatics 19, 12 (2003), 1572-1574.

[105] Rossi, P. V., Kabashima, Y., And Inoue, J.-I. Bayesian online compressed sensing. Physical Review E 94, 2 (2016), 022137.

[106] Rossi, P. V., And Vicente, R. L1-minimization algorithm for bayesian online compressed sensing. Entropy 19, 12 (2017), 667.

[107] SaAD, D., Ed. On-Line Learning in Neural Networks. Cambridge University Press, 1998.

[108] SaAd, D., AND Solla, S. A. Exact solution for on-line learning in multilayer neural networks. Phys. Rev. Lett. 74 (1995), 4337.

[109] Sakai, R., Sasaki, D., And Nakahashi, K. Large-scale cfd data compression for building-cube method using wavelet transform. In Computational Fluid Dynamics 2010: Proceedings of the Sith International Conference on Computational Fluid Dynamcs (ICCFD6) (2010), pp. 465-472.

[110] Santosa, F., And Symes, W. W. Linear inversion of band-limited reflection seismograms. SIAM Journal on Scientific and Statistical Computing 7, 4 (1986), 1307-1330.

[111] Sarvotham, S., Baron, D., Wakin, M., Duarte, M. F., And BaraNIUK, R. G. Distributed compressed sensing of jointly sparse signals. In Asilomar conference on signals, systems, and computers (2005), pp. 15371541.

[112] Schulz, A., Da Silva, E. A. B., And Velho, L. Compressive Sensing. Impa, 2009.

[113] Shannon, C. Communication in the presence of noise. Proc. Institute of Radio Engineers 37 (1949), 10-21.

[114] Shiraki, Y., And Kabashima, Y. Typical reconstruction limits for distributed compressed sensing based on ấ $\breve{S} 2$, 1-norm minimization and bayesian optimal reconstruction. Journal of Statistical Mechanics: Theory and Experiment 2015, 5 (2015), P05029.

[115] SiviA, D. S. Data analysis: a Bayesian tutorial. Oxford university press, 1996.

[116] Strohmer, T. Measure what should be measured: Progress and challenges in compressive sensing. IEEE Signal Processing Letters 19-12 (2012), 887-893. 
[117] Takhar, D., Laska, J., Wakin, M. B., Duarte, M. F., Baron, D., Sarvotham, S., Kelly, K., and Baraniuk, R. G. A new compressive imaging camera architecture using optical-domain compression. ISET/SPIE Computational Imaging IV 6065, 606509 (2006), 1.

[118] Taubman, D., and Marcellin, M. JPEG2000 Image Compression Fundamentals, Standards and Practice: Image Compression Fundamentals, Standards and Practice, vol. 642. Springer Science \& Business Media, 2012.

[119] Taylor, I. J., Deelman, E., Gannon, D. B., And Shields, M. Workflows for e-Science: Scientific Workflows for Grids. Springer Publishing Company, Incorporated, 2014.

[120] Tibshirani, R. Regression shrinkage and selection via the lasso. Journal of the Royal Statistical Society. Series B (Methodological) 58, 1 (1996), 267-288.

[121] Tramel, E. W., Gabrié, M., Manoel, A., Caltagirone, F., and Krzakala, F. A deterministic and generalized framework for unsupervised learning with restricted boltzmann machines. arXiv preprint arXiv:1702.03260 (2017).

[122] Tramel, E. W., Manoel, A., Caltagirone, F., Gabrié, M., And Krzakala, F. Inferring sparsity: Compressed sensing using generalized restricted boltzmann machines. In Information Theory Workshop (ITW), 2016 IEEE (2016), IEEE, pp. 265-269.

[123] Tropp, J. A. Greed is good: Algorithmic results for sparse approximation. IEEE Transactions on Information theory 50, 10 (2004), 2231-2242.

[124] VAn den Berg, E., And Friedlander, M. P. SPGL1: A solver for largescale sparse reconstruction, June 2007. http://www.cs.ubc.ca/labs/scl/spgl1.

[125] VAn den Berg, E., And Friedlander, M. P. Probing the pareto frontier for basis pursuit solutions. SIAM Journal on Scientific Computing 31, 2 (2008), 890-912.

[126] Van den Broeck, C., And Reimann, P. Unsupervised learning by examples: On-line versus off-line. Phys. Rev. Lett. 76 (1996), 2188.

[127] Vicente, R. Statistical Physics of Error-Correcting Codes. PhD thesis, Aston University, 2001.

[128] Vicente, R., Kinouchi, O., And Caticha, N. Statistical mechanics of online learning of drifting concepts: A variational approach. Machine Learning 32 (1998), 179.

[129] Vicente, R., SaAd, D., And Kabashima, Y. Low-density parity-check codes: A statistical physics perspective. Advances in Imaging and Electron Physics 125 (2002), 231-353. 
[130] Vicente, R., Susemihl, A., Jericó, J. P., And Caticha, N. Moral foundations in an interacting neural networks society: A statistical mechanics analysis. Physica A: Statistical Mechanics and its Applications 400 (2014), $124-138$

[131] WAINWRIGHT, M. J. Information-theoretic limits on sparsity recovery in the high-dimensional and noisy setting. IEEE Transactions on Information Theory 55, 12 (2009), 5728-5741.

[132] Wainwright, M. J. Sharp thresholds for high-dimensional and noisy sparsity recovery using $\ell_{1}$-constrained quadratic programming (lasso). IEEE transactions on information theory 55, 5 (2009), 2183-2202.

[133] Wakin, M. B., Laska, J. N., Duarte, M. F., Baron, D., Sarvotham, S., Takhar, D., Kelly, K. F., and Baraniuk, R. G. An architecture for compressive imaging. In Image Processing, 2006 IEEE International Conference on (2006), IEEE, pp. 1273-1276.

[134] Wakin, M. B., Sarvotham, S., Duarte, M. F., Baron, D., And BaraNIUK, R. G. Recovery of jointly sparse signals from few random projections. In In Advances in Neural Information Processing Systems 18 (NIPS 2005) (2005), pp. 1435-1442.

[135] Wang, C., And Lu, Y. M. Online learning for sparse pca in high dimensions: Exact dynamics and phase transitions. In Information Theory Workshop (ITW), 2016 IEEE (2016), IEEE, pp. 186-190.

[136] Xu, Y., And Kabashima, Y. Statistical mechanics approach to 1-bit compressed sensing. Journal of Statistical Mechanics: Theory and Experiment (2013), P02041.

[137] Xu, Y., Kabashima, Y., And Zdeborová, L. Bayesian signal reconstruction for 1-bit compressed sensing. J. Stat. Mech. Theor. Exp., 11 (2014), P11015.

[138] Yoshida, R., Imoto, S., And Higuchi, T. Estimating time-dependent gene networks from time series microarray data by dynamic linear models with markov switching. In Computational Systems Bioinformatics Conference, 2005. Proceedings. 2005 IEEE (2005), IEEE, pp. 289-298. 
And so it goes. 